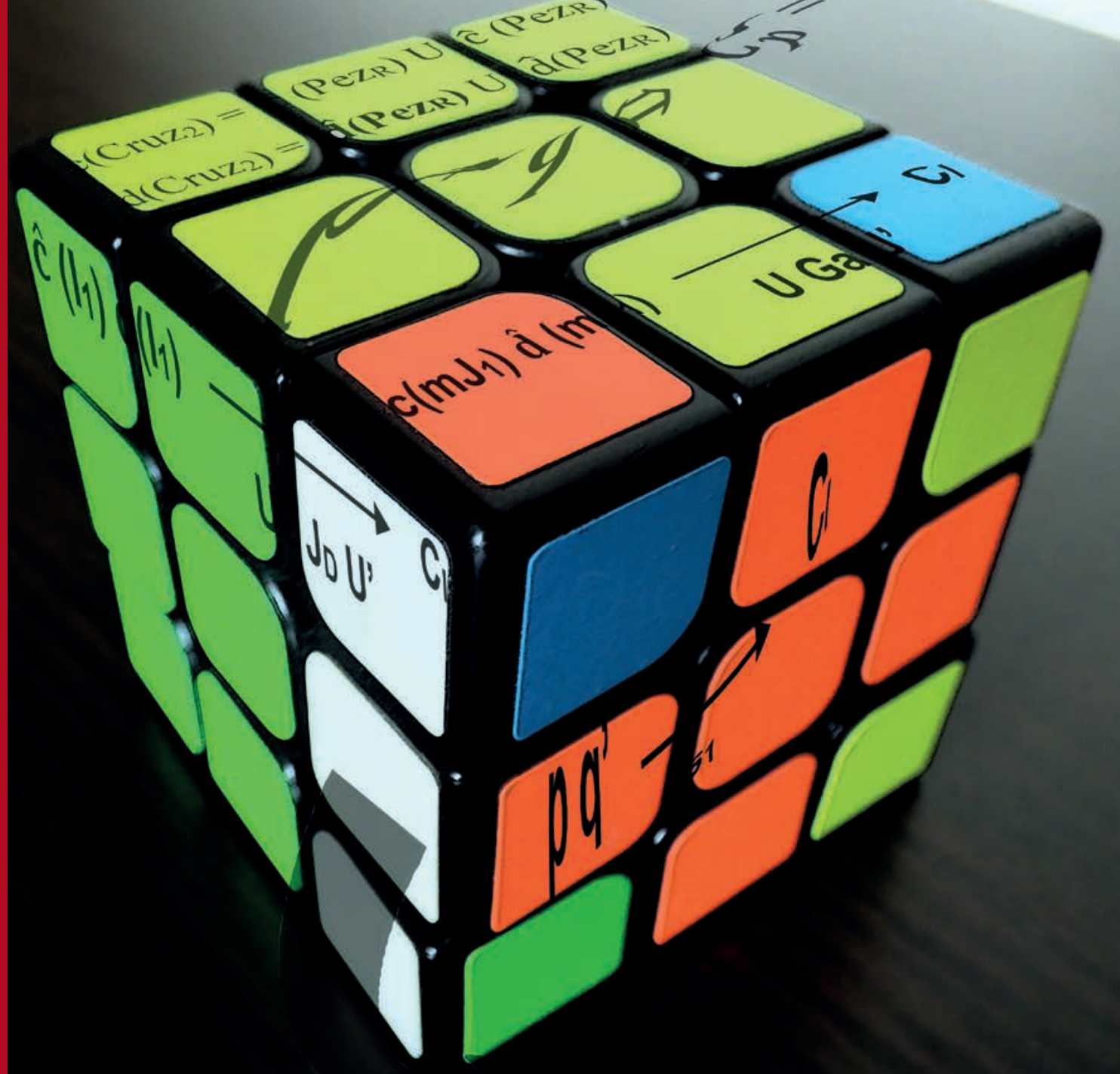

\title{
Bases y caminos algebraicos hacia los OLL/PLL del cubo Rubik
}

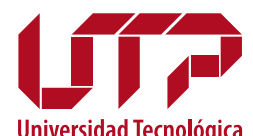

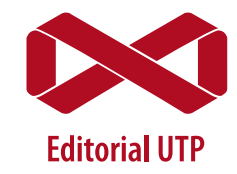

Julián Guzmán Baena Robin Mario Escobar Escobar César Alberto Mayoral Ramírez 
Julián Guzmán Baena, (Calarcá, Quindío, Colombia, 1955).

Magister en matemáticas y Matemático de la Universidad Nacional. Profesor titular de la facultad de Ciencias Básicas de la Universidad Tecnológica de Pereira.

Coautor del libro "960 Hexagramas Mágicos Puros ... Y No Más" (2018).

$\mathrm{Ha}$ publicado artículos en revistas especializadas nacionales e internacionales.

Pertenece al grupo categorizado por Colciencias, Investigación Estadística y Social (ISE).

Guzmanjulian34@gmail.com

Robin Mario Escobar Escobar, Itaguí, Antioquia, Colombia, 1974).

Magister en la Enseñanza de la Matemática y Licenciado en Matemáticas y física de la Universidad Tecnológica de Pereira. Profesor titular de la Facultad de Ciencias Básicas de la Universidad Tecnológica de Pereira.

Coautor del libro "960 Hexagramas Mágicos Puros ... Y No Más" (2018).

$\mathrm{Ha}$ publicado artículos en revistas especializadas nacionales e internacionales.

Pertenece al grupo categorizado por Colciencias, Investigación Estadística y Social (ISE).

romaes@utp.edu.co

César Alberto Mayoral Ramírez, (Florencia, Caquetá, Colombia, 1977).

Magister en la Enseñanza de la Matemática e ingeniero de Sistemas y computación de la universidad Tecnológica de Pereira. Profesor asociado de la Facultad de Ciencias Básicas de la Universidad Tecnológica de Pereira.

Coautor del libro "960 Hexagramas Mágicos Puros ... Y No Más"(2018).

$\mathrm{Ha}$ publicado artículos en revistas especializadas nacionales e internacionales.

Pertenece al grupo categorizado por Colciencias, Investigación Estadística y Social (ISE).

cmayoral@utp.edu.co

La Editorial de la Universidad Tecnológica de Pereira tiene como política la divulgación del saber científico, técnico y humanístico para fomentar la cultura escrita a través de libros y revistas científicas especializadas.

Las colecciones de este proyecto son: Trabajos de Investigación, Ensayos, Textos Académicos y Tesis Laureadas.

Este libro pertenece a la Colección Textos Académicos. 


\section{Bases y caminos algebraicos hacia los OLL/PLL del cubo Rubik}

Julián Guzmán Baena

Robin Mario Escobar Escobar

César Alberto Mayoral Ramírez

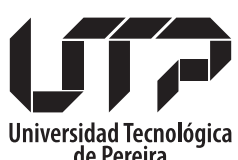

de Pereira

Colección Textos Académicos

Facultad de Ciencias Básicas

2020 
Guzmán Baena, Julián

Bases y caminos algebraicos hacia los OLL/PLL del cubo

Rubik / Julián Guzmán Baena, Robin Mario Escobar Escobar

y César Alberto Mayoral Ramírez. - Pereira : Universidad

Tecnológica de Pereira, 2020.

232 páginas. - (Colección Textos Académicos).

ISBN: 978-958-722-478-8

eISBN: 978-958-722-479-5

1. Matemáticas recreativas 2. Juegos matemáticos 3. Lógica

matemática 4. Análisis matemático 5. Algoritmos - Enseñanza

6. Matemáticas - Aprendizaje

CDD. 793.72

\section{Autores}

(C) Julián Guzmán Baena

(C) Robin Mario Escobar Escobar

(C) César Alberto Mayoral Ramírez

\section{Universidad Tecnológica de Pereira \\ Pereira, Colombia}

\section{Universidad Tecnológica de Pereira}

Vicerrectoría de Investigaciones, Innovación y Extensión

Editorial Universidad Tecnológica de Pereira

Pereira, Colombia

Coordinador editorial:

Luis Miguel Vargas Valencia

luismvargas@utp.edu.co

Teléfono 3137381

Edificio 9, Biblioteca Central "Jorge Roa Martínez"

Cra. 27 No. 10-02 Los Álamos, Pereira, Colombia

www.utp.edu.co

Montaje y producción:

David Restrepo Suarez.

Universidad Tecnológica de Pereira

Impresión y acabados: Gráficas Olímpica 



\section{CONTENIDO}

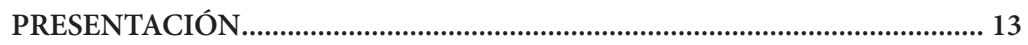

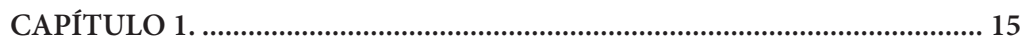

Bases Dinámicas del Cubo de Rubik ...................................................................... 16

1.1 Caras, sus elementos y componentes ........................................................ 16

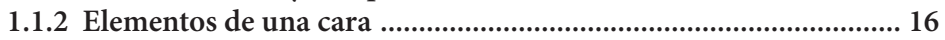

1.1.3 Componentes de una cara.................................................................. 17

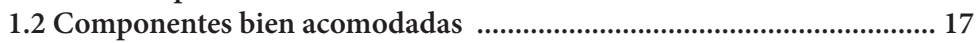

1.3 Capas de un cubo y sus movimientos fundamentales ...................... 18

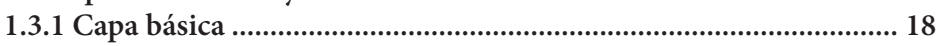

1.3.2 Capa central y su movimiento básico: ............................................ 20

1.4 Movimientos generales de un cubo de Rubik y algoritmos..................... 21

1.5 Algoritmos y Estructuras OLL ................................................................... 21

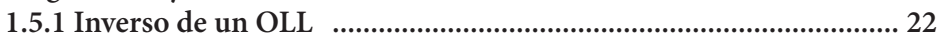

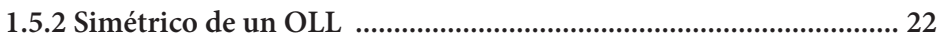

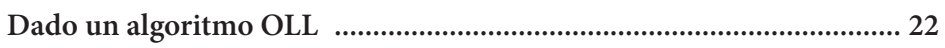

1.5.3 Configuración OLL ............................................................................ 23

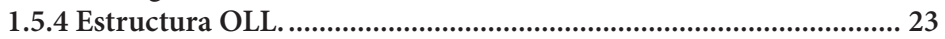

1.5.5 Inversa de una configuración OLL .................................................. 24

1.6 Nuestro primer OLL: La T

1.6.1 Configuración, constructor y deconstructor de " $\mathrm{T}_{1}$ " ....................... 24

1.6.2 Dinámica del constructor de $\mathrm{T}_{1}, \mathrm{c}\left(\mathrm{T}_{1}\right)$............................................ 25

$1.7 \mathrm{P}_{1}$ : El inverso de $\mathrm{T}_{1}$ o La Primera " $\mathrm{P}$ "........................................................ 28

1.7.1 Configuración, constructor y deconstructor de " $\mathrm{P}_{1}$ " ..................... 28

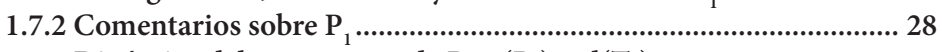

1.7.3 Dinámica del constructor de $\mathrm{P}_{1}, \mathrm{c}\left(\mathrm{P}_{1}\right)=\mathrm{d}\left(\mathrm{T}_{1}\right)$.............................. 29

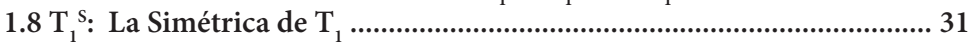

1.8.1 Configuración, constructor y de constructor de $\mathrm{T}_{1}^{\mathrm{S}}$........................ 31

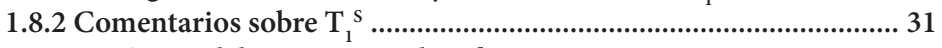

1.8.3 Dinámica del constructor $\mathrm{de}^{\mathrm{T}} \mathrm{T}_{1}^{\mathrm{s}}$...................................................... 32

$1.9 \mathrm{q}_{1}$ : El inverso del simétrico de $\mathrm{T}_{1}$ o La Primera " $\mathrm{q}$ " ................................. 34

1.9.1 Configuración, constructor y deconstructor de $\mathrm{q}_{1}$......................... 34

1.9.2 Comentarios sobre $\mathrm{q}_{1}$........................................................................ 34

1.9.3 Dinámica del constructor de $\mathrm{q}_{1}, \mathrm{c}\left(\mathrm{q}_{1}\right)=\mathrm{d}\left(\mathrm{T}_{1}{ }^{\mathrm{s}}\right)$............................ 35

1.10 Observaciones Numéricas Interesantes (Cubo original $3 \times 3 \times 3$ ).......... 37

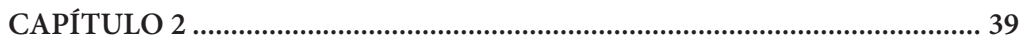

La Segunda T y Los Primeros siete PLL ............................................................... 40

2.1 La segunda $\mathrm{T}: \mathrm{T}_{2}$. Su inversa y su simétrica .........................................40

2.1.1 Configuración, constructor y deconstructor de " $\mathrm{T}_{2}$ " ...................... 40

2.1.2 Comentarios sobre $\mathrm{T}_{2}$...................................................................... 40

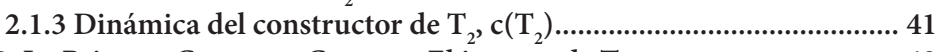

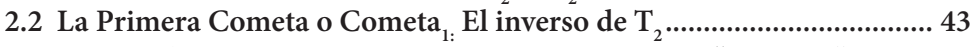

2.2.1 Configuración, constructor y deconstructor de "Cometa ${ }_{1}$ "............ 43

2.2.2 Dinámica del constructor de Cometa ${ }_{1}, \mathrm{c}\left(\right.$ Cometa $\left._{1}\right)$........................ 43 


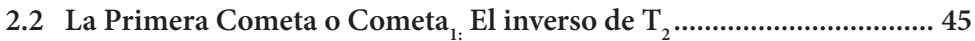

2.2.1 Configuración, constructor y deconstructor de "Cometa "............ 45

2.2.2 Dinámica del constructor de Cometa ${ }_{1}, \mathrm{c}\left(\right.$ Cometa $\left._{1}\right)$........................ 45

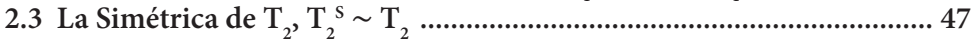

2.3.1Configuración, constructor y deconstructor de " $\mathrm{T}_{2}$ "

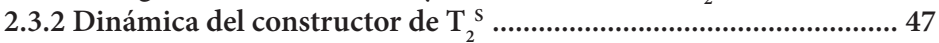

2.4 Cometa ${ }_{1}{ }^{\mathrm{S}}$ : $\quad$ EL inverso del simétrico de $\mathrm{T}_{2}$............................................. 49

2.4.1 Configuración, constructor y deconstructor de $\left(\mathrm{T}_{2}^{\mathrm{S}}\right)^{\prime}$..................... 49

2.4.2 Dinámica del constructor de Cometa ${ }_{1}^{\mathrm{S}}, \mathrm{c}\left(\left(\mathrm{T}_{2}^{\mathrm{S}}\right)^{\prime}\right)=\mathrm{d}\left(\mathrm{T}_{2}{ }^{\mathrm{S}}\right)$.............. 49

2.5 Las U: Permutaciones de 3 aristas con los vértices bien acomodados .. 51

2.5.1 Ua: Permutación de tres aristas en sentido horario......................... 51

2.5.2 Ub = Ua': Permutación de tres aristas en sentido antihorario. ..... 52

2.5.3 Configuración para aplicar los algoritmos " $U$ " ................................ 53

2.6 Las A: Permutaciones de 3 vértices con las aristas bien acomodadas... 54

2.6.1 Aa: Permutación de tres vértices en sentido horario....................... 54

2.6.2 Ab = Aa': Permutación de tres vértices en sentido antihorario...... 55

2.6.3 Configuración para aplicar los algoritmos "A" ................................ 56

2.7 La permutación " $\mathrm{H}$ ": Intercambiando aristas opuestas............................ 57

2.71 Motivación. $\mathrm{H}=\mathrm{Ub} \mathrm{U}$ Ub U' ........................................................... 57

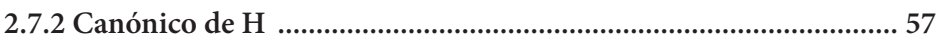

2.7.3 Configuración "Ajedrezada con opuestos", aplicar el algoritmo

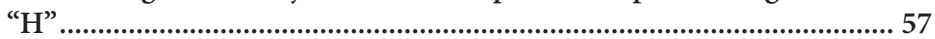

2.8 La permutación " $\mathrm{Z}$ ”: Intercambiando aristas contiguas ......................... 58

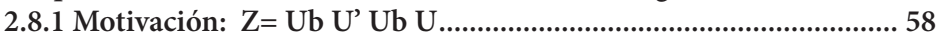

2.8.2 Canónico de $\mathrm{Z}$................................................................................. 59

2.8.3 Configuración "Ajedrezada de NO opuestos", aplicar el algoritmo

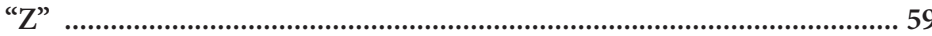

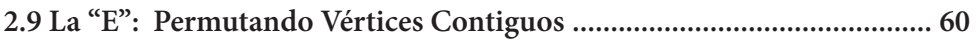

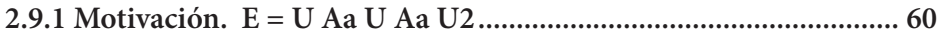

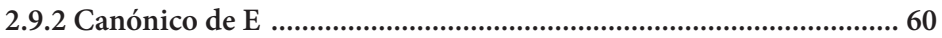

2.9.3 Configuración "Visualmente desordenada", aplicar el algoritmo

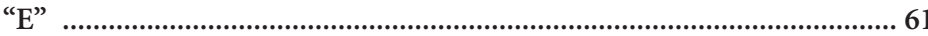

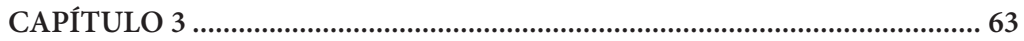

Operaciones OLL/PLL y Configuraciones semejantes ........................................ 64

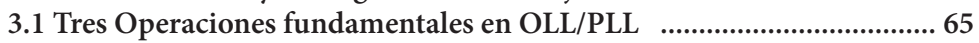

3.1.1 La operación Conjunción entre algoritmos OLL /PLL .................... 65

3.1.2 La operación externa $*$ entre configuraciones y algoritmos OLL.. 66

3.2 OLL Semejantes y clases de equivalencia por semejanza ........................ 66

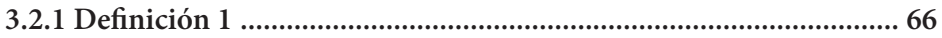

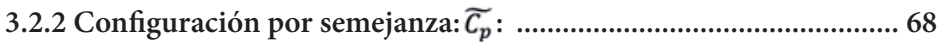

3.3 La semejanza: Una relación de equivalencia. Invarianza de la simetría

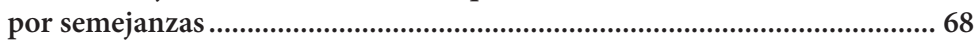

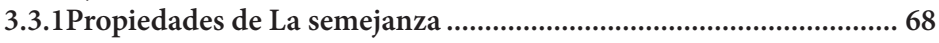

3.3.2 La conservación de la semejanza por simetría ............................... 69

3.4 Clases de equivalencias y algoritmos OLL canónicos ............................... 70

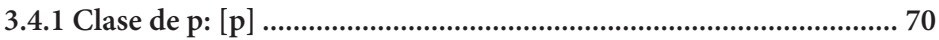




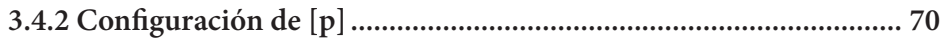

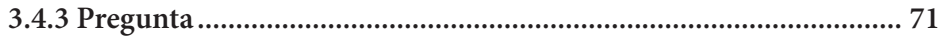

3.4.4 Propiedad fundamental del "canónico" de una clase ..................... 71

3.5 Configuración cuasi semejante y su nomenclatura indexada ................ 72

3.6 Congruencia de dos OLL/PLL. Configuraciones congruentes............... 73

3.6.3 Configuraciones OLL/PLL congruentes (o Idénticas)..................... 74

3.7 Nuevos constructor/deconstructor para $\mathrm{T}_{1}$............................................. 75

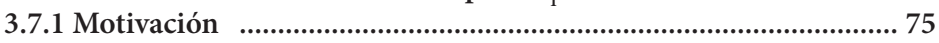

3.7.2 Dos formas de construir/deconstruir $\mathrm{T}_{1}$......................................... 76

3.7.3 La no congruencia de las dos formas ............................................... 76

3.7.4 La semejanza en la configuración de las dos formas ....................... 77

3.8 Nuevos constructor/deconstructor para $\mathrm{T}_{2}$............................................. 79

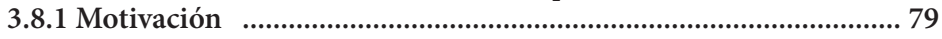

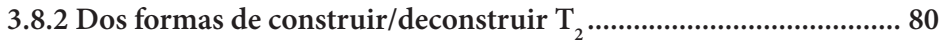

3.8.3 La no congruencia de las dos formas ................................................ 80

3.8.4 La semejanza en la configuración de las dos formas ........................ 80

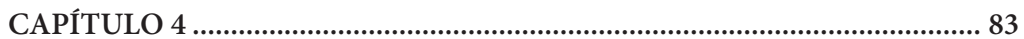

Las potencias dos $\mathrm{y}$ tres con $\mathrm{T}_{1}$ : "I " $\mathrm{y}$ "Cruz " ....................................................... 84

$4.1 \mathrm{I}_{1}$ : La Primera "I" y dos de sus constructores ............................................ 84

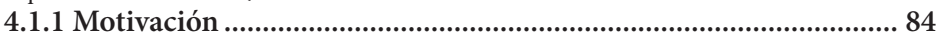

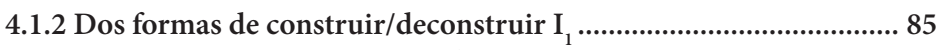

4.1.3 La no congruencia de las dos formas ............................................... 85

4.1.4 La semejanza en la configuración de las dos formas ....................... 85

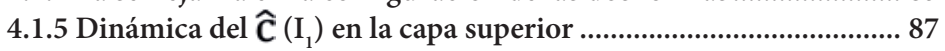

4.2 Nuevos constructor/deconstructor de $\mathrm{I}_{1}$. Los PLL "J" ............................. 88

4.2.1 La no congruencia de las dos formas .............................................. 88

4.2.2 La semejanza en la configuración de las dos formas ........................ 88

4.3 Las fascinantes permutaciones " $\mathrm{J}_{\mathrm{D}}$ " $\mathrm{y}$ " $\mathrm{J}_{\mathrm{I}}$ " .................................................... 90

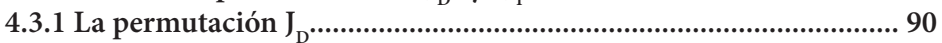

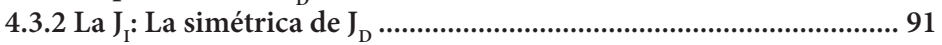

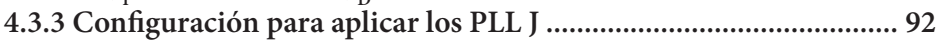

4.4 Las Permutaciones "N" .................................................................... 93

4.4.1 La permutación $\mathrm{N}_{\mathrm{D}}$........................................................................... 93

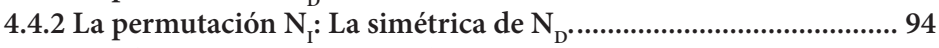

4.4.3 Configuración para aplicar los PLL N............................................... 95

$4.5 \mathrm{I}_{1}^{\mathrm{S}}$ : La simétrica de "I " y dos de sus constructores ................................... 96

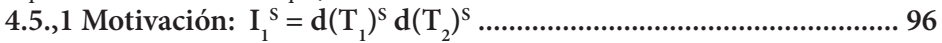

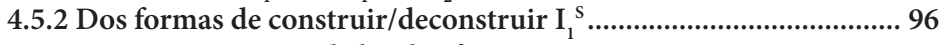

4.5.3 La no congruencia de las dos formas ............................................ 96

4.5.4 La semejanza en la configuración de las dos formas ...................... 97

4.5.5 Dinámica del $\hat{\mathbf{C}}\left(\mathbf{I}_{1}{ }^{\mathrm{S}}\right)$ en la capa superior......................................98

4.6 La Cruz ${ }_{1}$ en su forma canónica.................................................................. 99

4.6.1 Motivación $\mathbf{C r u z}_{1}=\hat{\mathbf{c}}\left(\mathbf{I}_{1}\right) \mathbf{d}\left(\mathbf{T}_{1}\right)$................................................99

4.6.2 Constructor/deconstructor canónicos de $\mathrm{Cruz}_{1}$.............................. 100

4.6.3 Dinámica del $\widehat{\mathbf{C}}\left(\mathrm{Cruz}_{1}\right)$ en la capa superior .................................... 100

4.7 Nuevos constructores/deconstructores de la Cruz ${ }_{1}$ El PLL V ............... 101

4.7.1 Dos formas de construir/deconstruir $\mathrm{Cruz}_{1}$.................................. 101 
4.7.2 La no congruencia de las dos formas ............................................. 101

4.7.3 La semejanza de la configuración de las dos formas ..................... 101

4.8 La Permutación "V": Su canónica y configuración................................ 103

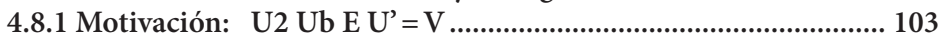

4.8.2 Forma canónica del PLL "V" ....................................................... 103

4.8.3 Configuración para aplicar el algoritmo " $\mathrm{V}$ "................................... 104

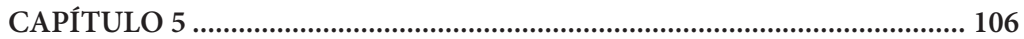

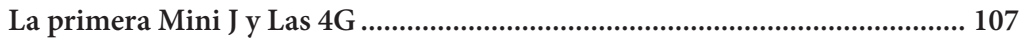

5.1 La Primera Mini J: $\mathrm{mJ}_{1}$ y su forma canónica ........................................ 107

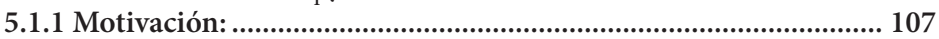

5.1.2 Constructor/deconstructor canónicos de “ $\mathrm{mJ}_{1}$ ” (= $\left.\mathrm{I}_{1}{ }^{\prime}\right)$................... 108

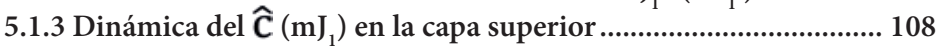

5.2 Un nuevo constructor de $\mathrm{mJ}_{1}$. Inicio de los PLL "Ga y Gb".................. 109

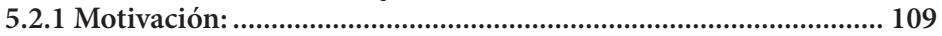

5.2.2 Nuevo constructor/deconstructor de $\mathrm{mJ}_{1}$ o $\mathrm{mJ}(2,1,1,2)$........... 109

5.2.3 La no congruencia de las dos formas ............................................ 110

5.2.4 La semejanza en la configuración de las dos formas .................... 110

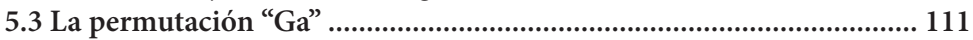

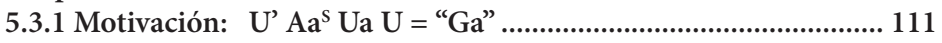

5.3.2 Objetivo de $\mathrm{Ga}$ (en la capa superior) ............................................. 111

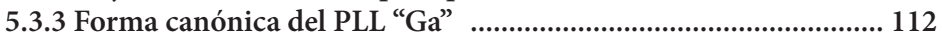

5.3.4 Configuración y Ayuda aplicativa de la permutación "Ga" ........ 112

5.4 La permutación "Gb": Inversa de Ga ................................................ 113

5.4.1 Motivación: U’ Ub AbS U = “Gb” ................................................. 113

5.4.2 Objetivo de Gb (en la capa superior).............................................. 113

5.4.3 Forma canónica del PLL "Gb" ......................................................... 114

5.4.4 Configuración y Ayuda aplicativa de la permutación “Gb” .......... 114

5.5 La permutación "Gd": Simétrica de "Ga" ................................................. 115

5.5.1 Motivación: U Aa Ub U’ = "Gd” ................................................ 115

5.5.3 Forma canónica del PLL "Gd" .................................................... 115

5.5.4 Configuración y Ayuda aplicativa de la permutación "Gd” ......... 116

5.6 La permutación "Gc": Simétrica de Gb .................................................. 117

5.6.1 Motivación: U Ua Ab U’ = “Gc” .................................................. 117

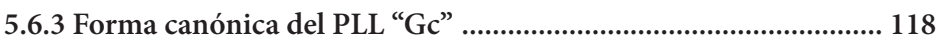

5.6.4 Configuración y Ayuda aplicativa de la permutación “Gc" ........ 118

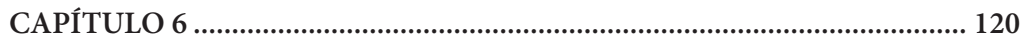

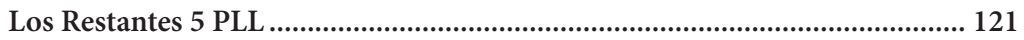

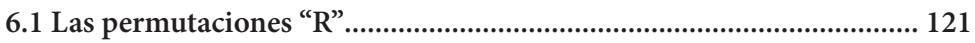

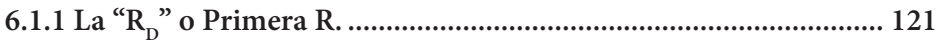

6.1.2 La " $\mathrm{R}_{\mathrm{I}}$ ” o Simétrica de la Primera $\mathrm{R}$.............................................. 122

6.1.3 Configuraciones para aplicar los algoritmos " $R$ " ........................... 123

6.2 Otro constructor de $\mathrm{mJ}_{1}$. Inicio de los PLL "T" y "F" .......................... 125

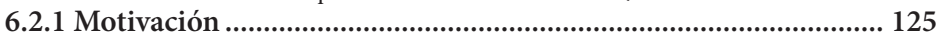

6.2.2 Nuevo constructor/deconstructor de $\mathrm{mJ}_{1} \mathrm{o} \mathrm{mJ}(2,1,1,2)$........... 125

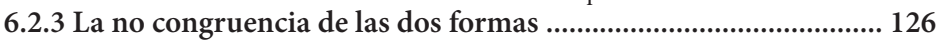

6.2.4 La semejanza en la configuración de las dos formas..................... 126 
6.3 La permutación "F"................................................................................... 127

6.3.1 Motivación: U’ Ab UZU = "F” ........................................................ 127

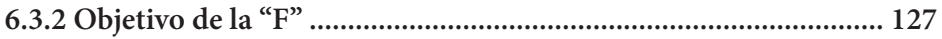

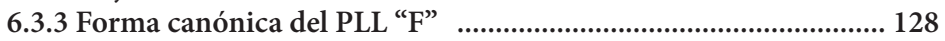

6.3.4 Configuración y Ayuda aplicativa de la permutación " $F$ " ............ 128

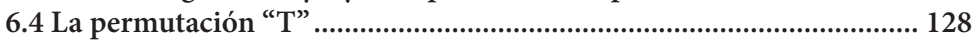

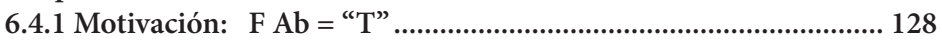

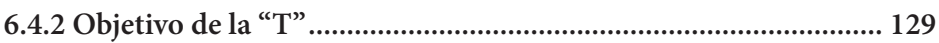

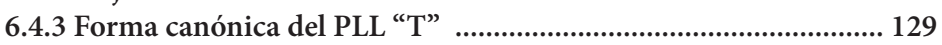

6.4.4 Configuración y ayuda aplicativa de la permutación “ $T$ ” ............ 129

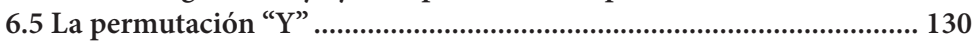

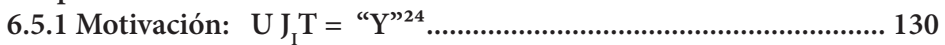

6.5.2 Objetivo de la "Y" ............................................................................... 130

6.5.3 Forma canónica del PLL "Y" ........................................................... 130

6.5.4 Configuración y Ayuda aplicativa de la permutación "Y" .......... 131

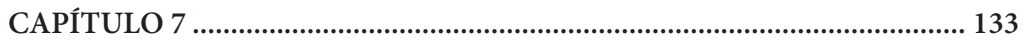

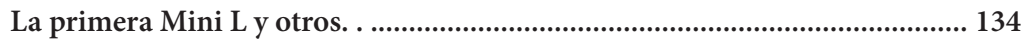

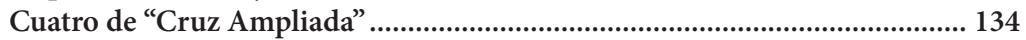

$7.1 \mathrm{~mL}_{1}$ : La Primera Mini L $(\mathrm{mL}(2,2,1,1))$ o Simétrica de $\mathrm{mJ}_{1} \ldots \ldots \ldots \ldots . . . . .135$

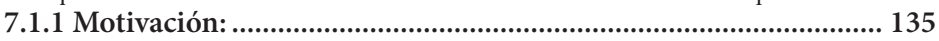

7.1.2 Constructor/deconstructor canónicos de " $\mathrm{mL}_{1}$ " $\left(=\mathrm{mJ}_{1}{ }^{\mathrm{S}}\right)$............ 135

7.1.3 Dinámica del $\widehat{\mathbf{C}}\left(\mathrm{mL}_{1}\right)$ en la capa superior ..................................... 136

7.1.3.1 Acción de $\widehat{\mathbf{C}}\left(\mathrm{mL}_{1}\right)$ sobre sus aristas iniciales.............................. 136

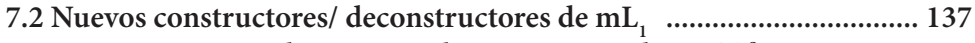

7.2.1 Constructor/deconstruir de $\mathrm{mL}_{1}$ a partir de $\mathrm{I}_{1} \mathrm{y} \mathrm{T}_{2}{ }^{\mathrm{s}}$. Sean: ......... 137

7.2.2 Constructor/deconstruir de $\mathrm{mL}_{1}$ a partir de $\mathrm{I}_{1}^{\mathrm{S}} \mathrm{y} \mathrm{T}_{2}^{\mathrm{s}}$ : Sean........ 137

7.3 Nuevo constructor/deconstructor de $\mathrm{Cruz}_{1}$ con la simétrica de $\mathrm{T}_{1} \mathrm{y}$

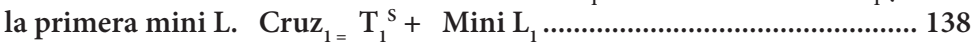

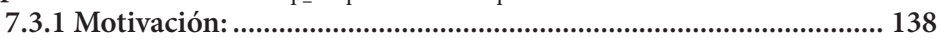

7.3.2 Nuevo constructor/deconstructor de la $\mathrm{Cruz}_{1}$................................ 138

7.4 La segunda Cruz: $\mathrm{Cruz}_{2}=\mathrm{I}_{1}+\mathrm{Mini}_{\mathrm{L}}$.................................................. 139

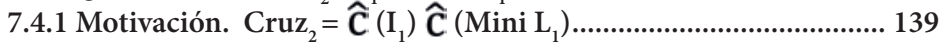

7.4.2 Dos formas de construir/deconstruir $\mathrm{Cruz}_{2}$.................................. 139

7.4.3 La no congruencia de las dos formas ............................................ 140

7.4.4 La semejanza de la configuración de las dos formas: ..................... 140

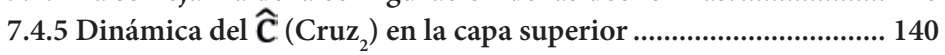

7.5 Nuevo constructor/deconstructor de $\mathrm{Cruz}_{2}$ con $\mathrm{mJ}_{1} \mathrm{y} \mathrm{T}_{2} \ldots \ldots \ldots \ldots \ldots \ldots . . . . . . .141$

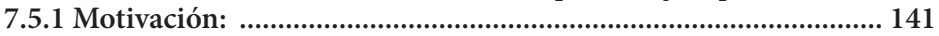

7.5.2 Nuevo constructor/deconstructor de la $\mathrm{Cruz}_{2}$.............................. 142

7.5.3 La no congruencia de las dos formas. ............................................ 142

7.5.4 La semejanza de la configuración de las dos formas: ................... 142

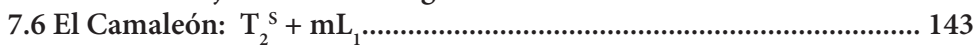

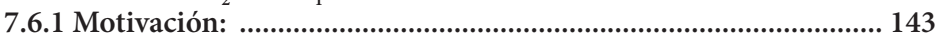

7.6.2 Dos formas de construir/deconstruir el Camaleón ....................... 144

7.6.3 La no congruencia de las dos formas ............................................. 144

7.6.4 La semejanza en la configuración de las dos formas: .................... 144

7.6.5 Dinámica del $\widehat{\mathbf{C}}$ (Camaleón) en la capa superior............................. 145 


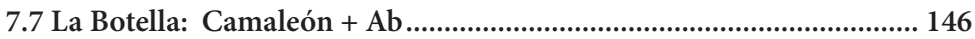

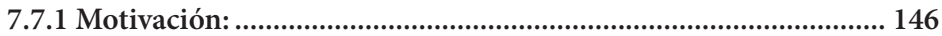

7.7.2 Dos formas congruentes de construir/deconstruir la Botella ..... 146

7.7.3 Dinámica del $\widehat{\mathbf{C}}$ (Botella) en la capa superior ............................... 147

7.8 Constructor/deconstructor de la Botella usando las " $\mathrm{T}$ ” ...................... 148

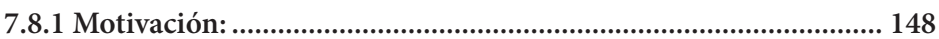

7.8.3 La no congruencia de las dos formas. ......................................... 149

7.8.4 La semejanza en la configuración de las dos formas: ................... 149

7.9 La Pajarita: Camaleón + Aa Inverso canónico del Camaleón ............... 150

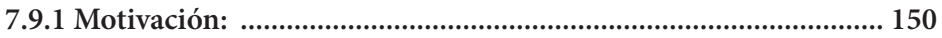

7.9.2 Dos formas de construir/deconstruir la Pajarita........................... 150

7.9.3 La no congruencia de las dos formas ........................................... 150

7.9.4 La semejanza de la configuración de las dos formas. .................... 150

7.9.5 Dinámica del $\widehat{\mathbf{C}}$ (Pajarita) en la capa superior.............................. 152

7.10 Constructor/deconstructor de la Pajarita usando las " $T$ ”.................. 153

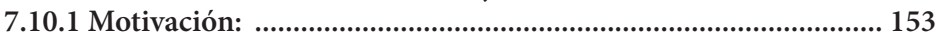

7.10.2 Nuevo constructor/deconstructor de la Pajarita ........................... 153

7.10.3 La no congruencia de las dos formas .......................................... 154

7.10.4 La semejanza de la configuración de las dos formas ................... 154

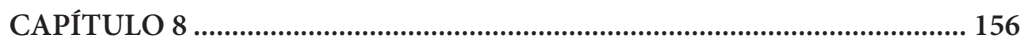

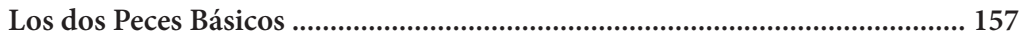

Completando el Grupo de "Las 7 cruces ampliadas" ........................................ 157

8.1 El Primer Pez Básico: $\mathrm{Pez}_{\mathrm{R}}$................................................................... 157

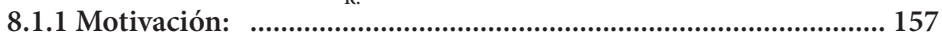

8.1.2 Dos Formas de construir/deconstruir PezR ................................. 158

8.1.3 La no congruencia de las dos formas ............................................. 158

8.1.4 La semejanza en la configuración de las dos formas: ................... 158

8.1.5 Dinámica del $\hat{\mathbf{c}}\left(\mathrm{Pez}_{\mathrm{R}}\right)$ en la capa superior ................................... 159

8.2 El segundo Pez Básico: $\mathrm{Pez}_{\mathrm{L}}$ ó Simétrico de $\mathrm{Pez}_{\mathrm{R}}$ …………………........ 160

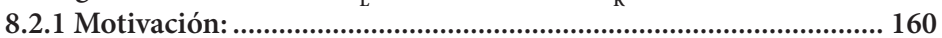

8.2.2 Dos Formas de construir/deconstruir PezL.................................. 160

8.2.3 La no congruencia de las dos formas ........................................... 161

8.2.4 La semejanza en la configuración de las dos formas: .................... 161

8.2.5 Dinámica del $\widehat{\mathbf{C}}\left(\mathrm{Pez}_{\mathrm{L}}\right)$ en la capa superior..................................... 161

8.3 Construir/deconstruir las "Cruces ampliadas" a partir de los Peces

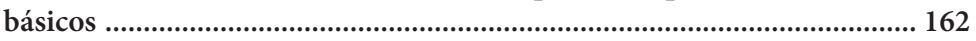

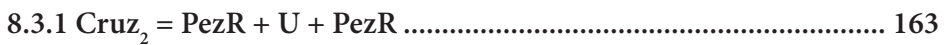

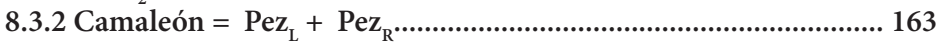

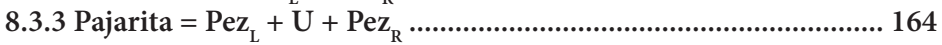

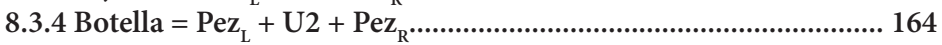

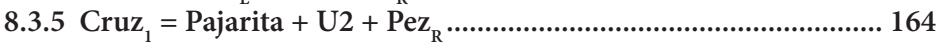

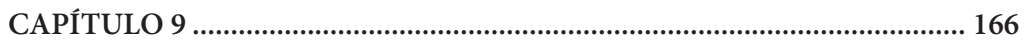

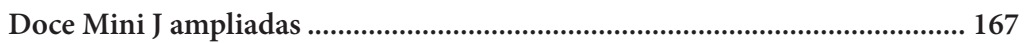

Optimizando Tiempo en La Resolución del Cubo de Rubik: Parte $1^{\text {a }}$............ 167

9.1 Clasificación de las restantes configuraciones OLL ................................ 167 


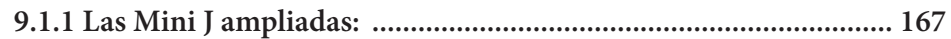

9.1.2 Las Mini L ampliadas: .................................................................... 168

9.1.3 Las Barras Centrales Ampliadas: ................................................... 168

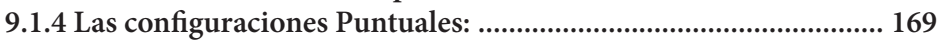

9.2 Cinco primeras "Mini J ampliadas" ........................................................ 170

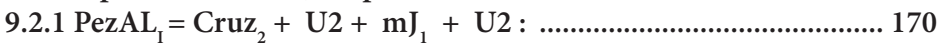

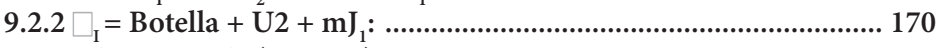

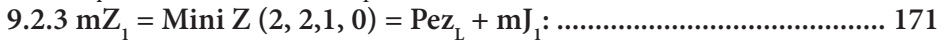

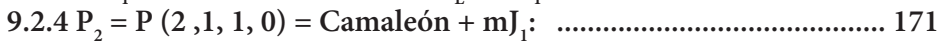

9.2.5 $\mathrm{CY}_{1}=$ Cuasi $_{1}(0,2,1,1)=\mathrm{Cruz}_{1}+\mathrm{U}+\mathrm{mJ}_{1}+\mathrm{U}$ ': ...................... 172

9.2.6 Las inversas de esas cinco configuraciones ................................... 173

9.3 Otras Cuatro "Mini J Ampliadas"............................................................ 173

9.3.1 $\mathrm{mS}_{2}=\operatorname{Mini} \mathrm{S}(1,2,1,1)=$ Camaleón $+\mathrm{U}+\mathrm{mJ}_{1}+\mathrm{U}$ ' : ............... 173

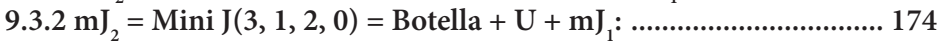

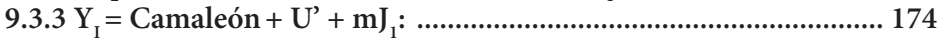

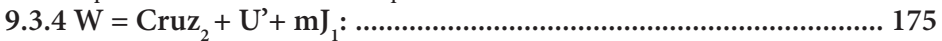

9.3.5 Las inversas de esas cuatro configuraciones .................................. 175

9.4 Las restantes Tres "Mini J Ampliadas"................................................ 176

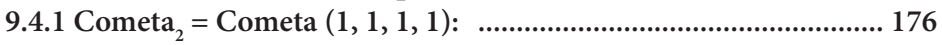

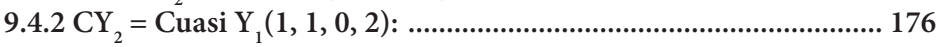

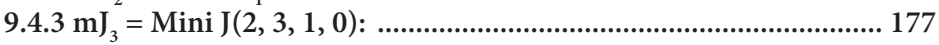

9.4.4 Las inversas de esas tres configuraciones ........................................... 177

9.5 Práctica: Las Mini J “Ampliadas” a partir de las “Cruces ampliadas”. 178

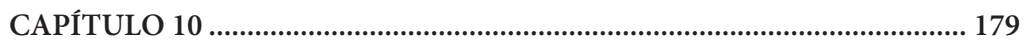

Doce Mini L ampliadas o simétricas ................................................................. 180

de las Mini J ampliadas......................................................................................... 180

Optimizando Tiempo en La Resolución del Cubo de Rubik: Parte 2a $2^{\text {a }}$........... 180

10.1 Cinco primeras "Mini L ampliadas" ................................................... 180

10.1.1 PezAL $_{\mathrm{D}}=\mathrm{Cruz}_{2}+\mathrm{mL}_{1}+\mathrm{U} 2:$................................................. 180

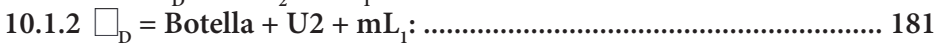

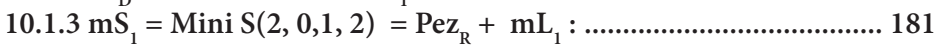

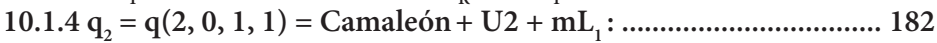

10.1.5 $\mathrm{CY}_{3}=$ Cuasi $\mathrm{Y}_{3}(0,1,1,2)=\mathrm{Cruz}_{1}+\mathrm{U}+\mathrm{mL}_{1}+\mathrm{U}: \ldots \ldots \ldots \ldots \ldots \ldots . . .182$

10.1.6 Las inversas de esas cinco configuraciones ................................... 183

10.2 Otras cuatro "Mini L Ampliadas" ........................................................... 184

10.2.1 $\mathrm{mZ}_{2}=\operatorname{Mini} \mathrm{Z}(1,1,1,2)=$ Camaleón $+\mathrm{U}+\mathrm{mL}_{1}+\mathrm{U}:$................ 184

10.2.2 $\mathrm{mL}_{2}=$ Mini $\mathrm{L}(3,0,2,1)=$ Botella $+\mathrm{U}^{\prime}+\mathrm{mL}_{1}: \ldots \ldots \ldots \ldots \ldots \ldots \ldots \ldots \ldots . . . . . . . . . .184$

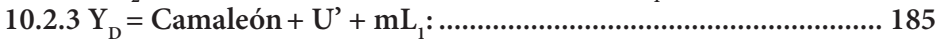

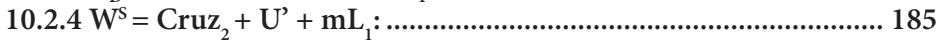

10.3.1 La Cometa ${ }_{2}{ }^{\mathrm{s}}$ : "Simétrica de la Cometa " (9.4.1) .......................... 187

10.3.2 $\mathrm{CY}_{4}=$ Cuasi $\mathrm{Y}_{3}(1,2,0,1)$ : "La Cuarta Cuasi Y" ........................... 187

10.3.3 $\mathrm{mL}_{3}=$ Mini L $(2,0,1,3)$ : La tercera Mini L ................................. 187

10.4 Práctica: Las Mini L "Ampliadas" a partir de las "Cruces

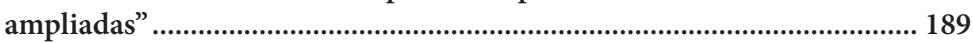

10.5 Sección Complementaria: .................................................................. 189 


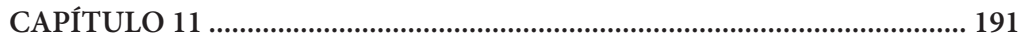

Doce Barras Centrales Ampliadas ................................................................... 192

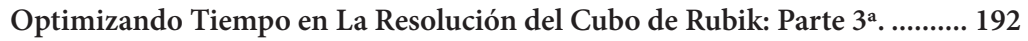

11.1 Cinco primeras "Barras Centrales Ampliadas".................................. 192

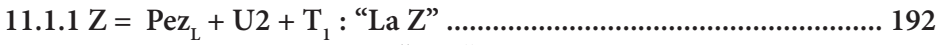

11.1.2 S = Camaleón + U + $\mathrm{T}_{1}$ : "La S" ...................................................... 193

11.1.3 $\mathrm{L}_{1}=\mathrm{L}(0,2,1,2)=\mathrm{Cruz}_{2}+\mathrm{U}+\mathrm{T}_{1}+\mathrm{U}^{\prime}:$........................................ 193

11.1.4 $\mathrm{J}_{1}=\mathrm{J}(0,2,1,2)=\mathrm{Cruz}_{2}+\mathrm{U}^{\prime}+\mathrm{T}_{1}+\mathrm{U}^{\prime}:$..................................... 194

11.1.5 $\mathrm{I}_{3}=\mathrm{I}(1,2,1,2)=$ Camaleón $+\mathrm{T}_{1}$ : ............................................... 194

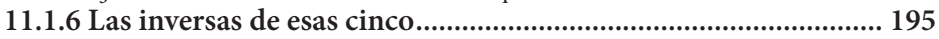

11.2 Otras cuatro "Barras Centrales Ampliadas" ...................................... 195

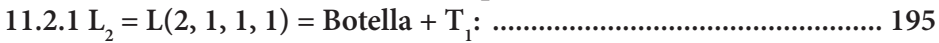

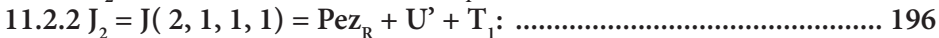

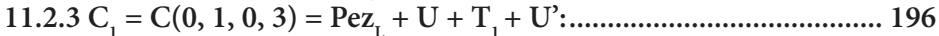

11.2.4 $\mathrm{C}_{2}=\mathrm{C}(1,1,1,1)=$ Pajarita $+\mathrm{U}^{\prime}+\mathrm{T}_{1}+\mathrm{U}^{\prime}:$..................................... 197

11.3 Las restantes Tres "Barras Centrales Ampliadas" ............................... 199

11.3.1 $\mathrm{I}_{2}=\mathrm{I}_{\mathrm{v}}(0,3,0,3)=$ "La Segunda I" ............................................... 199

11.3.2 $\mathrm{I}_{4}=\mathrm{I}_{\mathrm{V}}(1,3,1,1)$ : "La Cuarta I" .................................................... 199

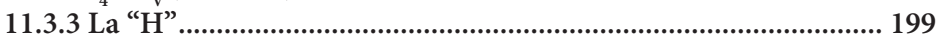

11.3.5 Práctica: Las "Barras centrales Ampliadas" a partir de Las

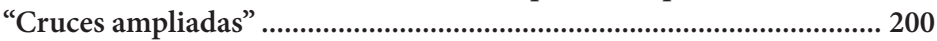

11.4 Sección Complementaria:.................................................................... 201

11.4.1 Nuevos contructor/decontructor de la configuración H............. 201

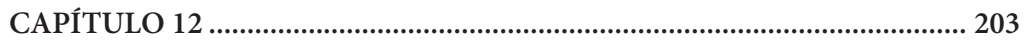

Ocho configuraciones Puntuales ....................................................................... 204

Optimizando Tiempo en La Resolución del Cubo de Rubik: Parte $4^{\text {a }}$............ 204

12.1 La "X": Base de las demás Puntuales ............................................. 204

12.2 El primer Punto y Su Inverso ... otro Punto ................................ 205

12.2.1 Punto $_{1}=$ Punto $(1,3,1,3)=\mathrm{Cruz}_{1}+\mathrm{U}+\mathrm{X}:$............................... 205

12.2.2 El Otro Punto: El inverso canónico del Punto ${ }_{1}$......................... 206

$12.3 \mathrm{D}_{\mathrm{NP1}}$ : La Primera Diagonal No Principal y su inversa $\mathrm{V}_{1}$................... 206

12.3.1 $\mathrm{D}_{\mathrm{NP1}}=\mathrm{D}_{\mathrm{NP}}(1,1,2,2)=$ Pajarita $+\mathrm{U} 2+\mathrm{X}:$............................... 206

12.3.2 $\mathrm{V}_{1}$ : La primera $\mathrm{V}$ o Inversa de la Primera Diagonal NO

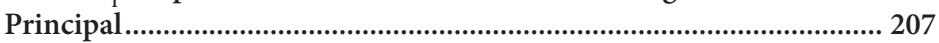

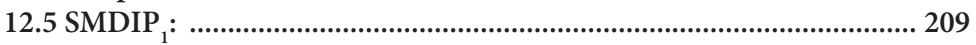

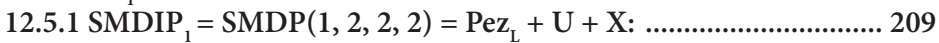

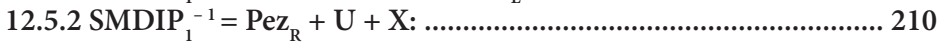

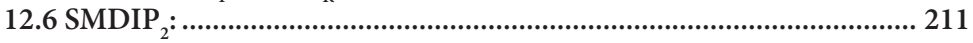

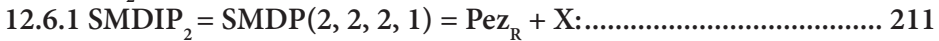

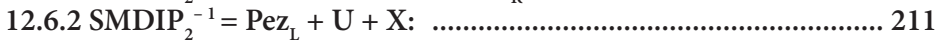

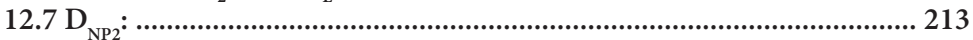

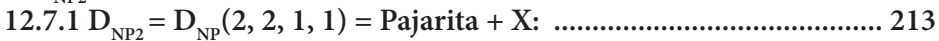

12.7.2 $\mathrm{V}_{2}=\mathrm{V}(1,2,1,2)=$ Camaleón $+\mathrm{U}^{\prime}+\mathrm{X}:$..................................... 214

12.8 Las "Configuraciones Puntuales" a partir de las "Cruces

ampliadas" ....................................................................................................... 216 



\section{PRESENTACIÓN}

El presente libro pretende realizar un compendio de los resultados del análisis matemático delos movimientos yalgoritmos, tanto OLL(Orientation of Last Layer: Orientación de la última capa), como PLL(Permutation of Last Layer: Permutación de la última capa), realizados sobre la última capa en el cubo de Rubik $3 \times 3 \times 3$, como un verdadero objeto de Enseñanza - Aprendizaje. Es resultado de una investigación aplicada en estudiantes de la Licenciatura de Matemáticas, como alternativa didáctica en asignaturas tales como Teoría de Grupos, Teoría de Números y Matemáticas Recreativas, con una duración aproximada de dos años, en los que se realizó la búsqueda de información, desarrollo intuitivo, formalización, pruebas de campo con los estudiantes, recolección de resultados, entre otros.

La presente obra inicia en el capítulo uno con un recorrido por los elementos básicos del cubo de Rubik, el cual es un rompecabezas mecánico, inventado por el escultor y arquitecto húngaro Ernő Rubik en el año 1974 y que ha despertado interés 
alrededor de todo el mundo por décadas, tanto en chicos como grandes. En este capítulo se describen conceptos como el de cara, centro, vértice, arista, torsión y los movimientos básicos, con la respectiva notación que fue desarrollada por el estadounidense David Singmaster en el año de 1981.

Como ya se planteó anteriormente. la investigación se enfoca en el estudio de los algoritmos OLL y PLL, que se desarrollan en la última capa del cubo Rubik, por lo cual se deja como tarea para el lector el aprendizaje de la realización correcta de las capas inferior e intermedia (se espera que el lector conozca como mínimo el método principiante para la realización del cubo de Rubik), es por esto que el primer capítulo finaliza con la construcción y análisis del primer OLL, denominado la $\mathrm{T}_{1}$, su inverso, su simétrico y el inverso del simétrico. El capítulo 2 analiza la segunda $\mathrm{T}$ (denominada $\mathrm{T}_{2}$ ) y los primeros 7 PLL. El capítulo 3 y 4 se refieren a las operaciones, configuraciones semejantes y potencias de algunos OLL/PLL. Los capítulos 5 al 12 hacen referencia a la construcción de OLL/PLL puntuales donde se resaltan versiones básicas y ampliadas de las Mini J, las Mini L, las Cruces, las barras centrales además de las 4G, Los Peces Básicos y los puntos.

Por último, pero no menos importante, cabe recalcar que la fuerza motriz cognitiva de esta obra es lo algebraico: las permutaciones y sus combinaciones, aunadas a los conceptos inherentes a lo inverso, lo simétrico, los patrones y algoritmos descritos por las variables asociadas a los movimientos básicos; además, en esencia, se desea brindar bases que lleven de un modo practico el desarrollo de los algoritmos planteados, los cuales son guiados por el álgebra y el método deductivo. Por otro lado, la estadística y otros conocimientos matemáticos derivados del cubo de Rubik o aplicados con este son sumamente interesantes, pero bien debe comprenderse que este libro es apenas una introducción al cubo en su avanzado estudio y, por ende, no hay espacio para que se incluyan tópicos distintos al algebraico. En un futuro, tal vez, trabajando directamente con OLL, se podrá hacer un trabajo didáctico, más pormenorizado donde lo sencillo y lo complejo se fusionen directamente para un profundo conocimiento de este bello objeto. 


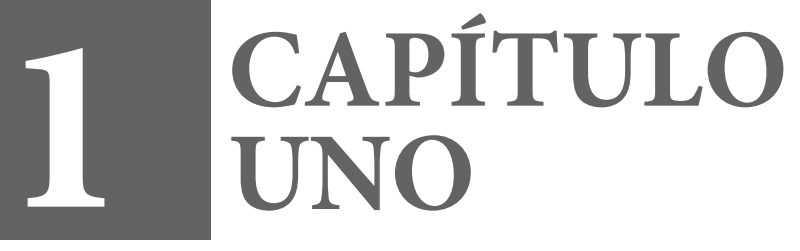




\section{Bases Dinámicas del Cubo de Rubik}

Se inicia este libro dando una serie de conceptos fundamentales en la buena comprensión, estudio y análisis del cubo de Rubik como un verdadero objeto de aprendizaje. Conceptos como los de caras y sus componentes, capas y sus movimientos básicos, movimientos en general y algoritmos (OLL y PLL) no pueden faltar en el buen conocimiento del cubo de Rubik; por ello, se ha incluido como inicio de la presente obra.

\subsection{Caras, sus elementos y componentes}

\subsubsection{Cara}

Cuadrado de $3 \times 3$ cubitos, todos ellos del mismo color cuando el cubo de Rubik está en su estado original. Son 6 en total y se denotan con las letras:

- U (Up): Cara superior

- D (Down): Cara Inferior

- R (Right): Cara Derecha

- L (Left): Cara Izquierda

- F (Front): Cara Frontal

- B (Back): Cara Trasera

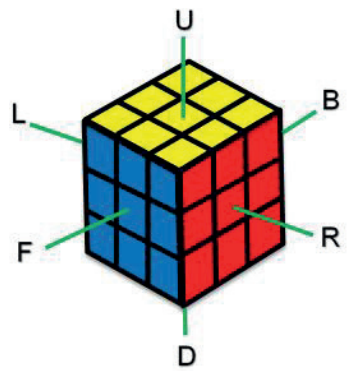

Y se clasifican en pares de caras opuestas: (F, B), (U. D) y (L, R)

\subsubsection{Elementos de una cara}

Toda cara tiene un centro, 4 vértices y 4 aristas, como se ilustra en siguiente figura:

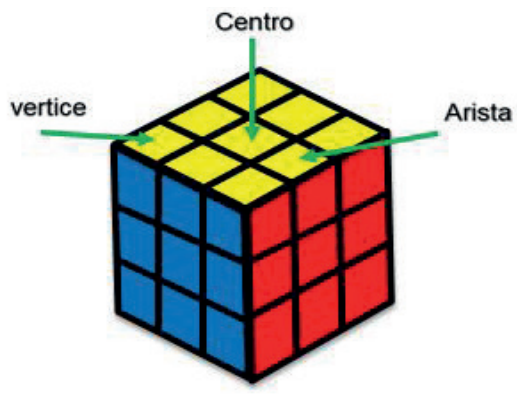


- Centro: Es el cubito de la mitad de la cara, muy clave pues siempre nos indica el color de la cara en la posición original del cubo. El cubo consta de 6 centros.

- Vértice: Es el cubito en la esquina de una cara, pero también de otras dos. Consta de tres colores (uno por cara). El cubo consta de 8 vértices.

- Aristas: Son las piezas que están entre los vértices y no hacen esquina. Hay 12 aristas en el cubo, cada una con dos colores distintos (2 stickers). Nótese que cada lado del cubo tiene una única pieza de este tipo, por ello el nombre de arista ("lado").

\subsubsection{Componentes de una cara}

Por convención en este libro las componentes de una cara son sus vértices y aristas (los centros de las caras en la práctica no los podemos mover).

\subsection{Componentes bien acomodadas}

Se dice que una componente de una cara está bien acomodada si y solo si se cumple una de las siguientes posibilidades:

a) Está bien posicionada: En el momento en que la observamos ocupa la posición que inicialmente tiene en el cubo en su estado original

b) No tiene torsión: Una componente de la cara tiene torsión en el momento de observación si al ubicar esta cara como superior se tienen dos hechos:

b.1 La componente está en esa cara

b.2 Si el sticker de la cara superior en esa componente se observa en otra cara (es como si en su posición original de la pieza se hubiera girado dicho sticker; y luego por rotación, si está mal posicionada la componente se hubiera llevado a otro lugar). 
De acuerdo con la cara donde se halla el sticker girado del mismo color de la cara superior hay cuatro torsiones para la componente: izquierda, derecha, frontal y trasera y las representaremos gráficamente así:

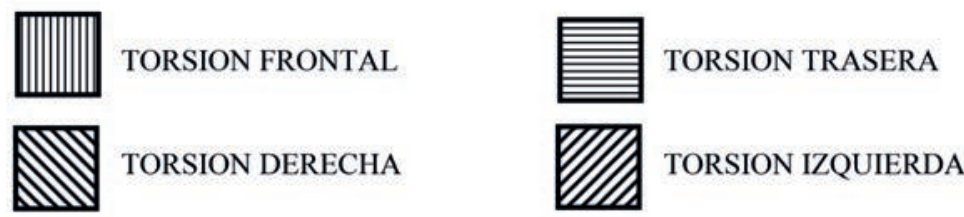

1.3 Capas de un cubo y sus movimientos fundamentales

\subsubsection{Capa básica}

La constituyen los 9 cubitos de una cara: sus cuatro aristas (consecutivas), sus cuatro vértices y un centro. Son en total 6 las capas básicas y se notan usando las letras U, D, R, L, F y B similares a las empleadas para las caras

\subsubsection{Movimiento básico de una capa del cubo de Rubik}

Es la rotación de $90^{\circ}$ sentido horario o antihorario de una capa. Los movimientos básicos se notan usando las mismas letras de las capas. Esta notación fue planteada en Singmaster(1981), por el estadounidense David Singmaste ${ }^{1}$

Figura I.David Singmaster,

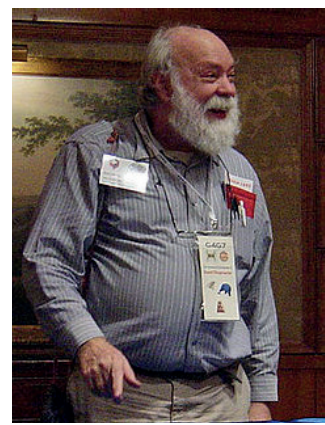

Fuente Wikipedia

1 https://es.wikipedia.org/wiki/David_Singmaster 
Comentario: Cuando una letra es seguida por una prima, indica un movimiento en el sentido contrario a las agujas del reloj, mientras que una letra sin prima indica un movimiento en sentido de las agujas del reloj. Una letra( $X)$ precedida por un 2 (X2) indica dos giros (de un mismo tipo), o un giro de $180^{\circ}$.
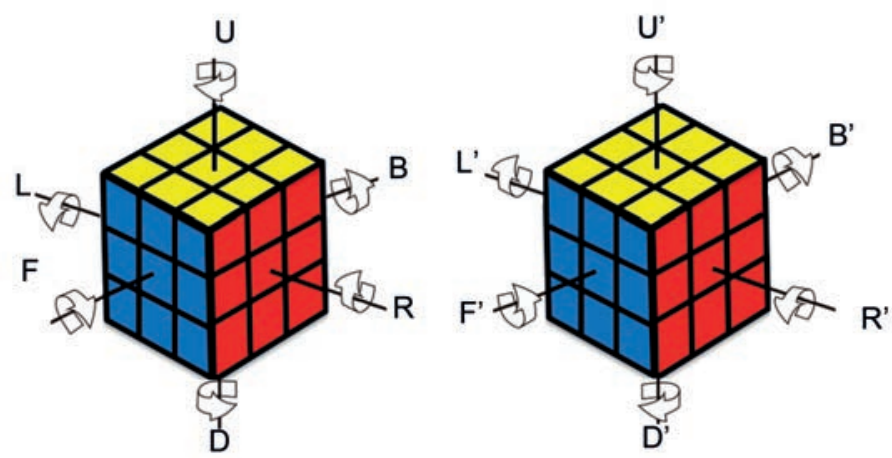

Observación: El movimiento " $y$ ” consiste en girar todo el cubo $90^{\circ}$ en sentido horario muy similar al movimiento U (solo que acá se gira todo el cubo y no sólo la capa superior). En caso de que el giro de todo el cubo sea en sentido antihorario se habla del movimiento y'.

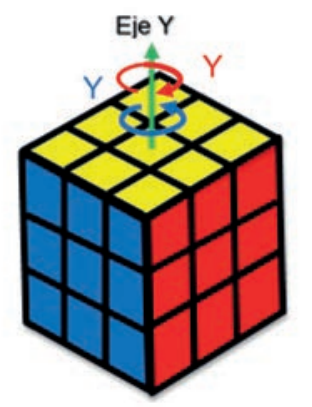

1.3.1.2 Movimientos simétricos de los básicos con respecto a los lados derecho e izquierdo

Actuar simétricamente en el cubo de Rubik es "Hacer con la mano izquierda lo que se hace con la mano derecha y viceversa"; las siguientes son las parejas de movimientos simétricos con respecto a los lados derecho (R) e izquierdo (L):

$$
\text { (R, L'), (R', L), (U, U'), (D, D'), (F, F'), (B, B'), }
$$


Usando la notación $\mathrm{A}^{\mathrm{S}}$ para el simétrico de A tenemos:
$\mathrm{R}^{\mathrm{S}}=\mathrm{L}^{\prime}$
$\left(\mathrm{R}^{\prime}\right)^{\mathrm{S}}=\mathrm{L}$
$(\mathrm{L})^{\mathrm{S}}=\mathrm{R}^{\prime}$
$\left(L^{\prime}\right)^{S}=\mathrm{R}$
$\mathrm{U}^{\mathrm{S}}=\mathrm{U}^{\prime} \quad\left(\mathrm{U}^{\prime}\right)^{\mathrm{S}}=\mathrm{U}$
$(\mathrm{D})^{\mathrm{S}}=\mathrm{D}^{\prime}$
$\left(D^{\prime}\right)^{S}=\mathrm{D}$
$\mathrm{F}^{\mathrm{S}}=\mathrm{F}^{\prime}$
$\left(F^{\prime}\right)^{S}=F$
$(B)^{S}=B^{\prime}$
$\left(B^{\prime}\right)^{S}=B$
$(y)^{S}=y^{\prime}$
$\left(y^{\prime}\right)^{S}=y$

\subsubsection{Capa central y su movimiento básico:}

Una capa central la constituyen 8 cubitos: 4 aristas (dos en una cara y dos en la cara opuesta) y los 4 centros. Son en total 3 y se notan usando las letras M, E y S.

M

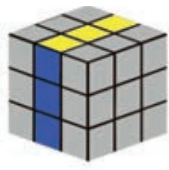

E

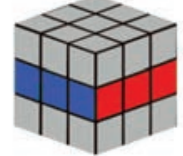

s

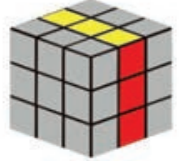

Capa "M": Esta capa se sitúa entre las capas "L" $y$ " $R$ " y gira para el mismo sentido que la capa "R".

Capa “E”: Está capa se sitúa entre las capas “D” y "U” y gira para el mismo sentido que la capa "U".

Capa "S": Está capa se sitúa entre las capas " $\mathrm{B}$ " y " $F$ " y gira para el mismo sentido que la capa "F".

\section{Comentario: $M^{\mathbf{S}}=\mathbf{M}$ y $\left(M^{\prime}\right)^{\mathbf{S}}=M^{\prime}$}

$M$ es auto-simétrico (el simétrico de $M$ es el mismo $M$ ) y lo mismo sucede con M'.

Antes de proseguir con el desarrollo del texto vale la pena resaltar que, aunque existen innumerables variaciones del cubo $3 \times 3$, el presente documento se basa en el desarrollo y análisis de los algoritmos para la última capa, del cubo original $3 \times 3$ desarrollado por Ernő Rubik (ver figura 2), el cual tiene como colores en sus caras el Blanco, Rojo, Naranja, azul, verde y amarillo. No obstante, todos los procesos y algoritmos desarrollados en el presente libro son válidos para cualquier cubo de Rubik de tamaño $3 \times 3 \times 3$, pues los movimientos básicos se dan y son los mismos en cada uno de estos. 
Figura 2.

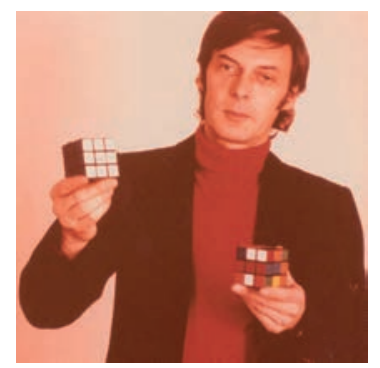

Ernő Rubik 1974, (https://co.pinterest.com/pin/384776361886848505/)

\subsection{Movimientos generales de un cubo de Rubik y algoritmos}

Los movimientos del cubo de Rubik son sucesiones de movimientos básicos(rotaciones de sus capas); yson fundamentales para llevar al cubo a su estado original (si está desordenado) o hacer configuraciones en el mismo. Esos movimientos se notan como: $\mathbf{A}_{1} \mathbf{A}_{2} \mathbf{A}_{3} \ldots \mathbf{A}_{\mathbf{n}}$.

donde cada $A_{i}$ es un movimiento básico (U, U’, D, D', R, R'... etc.)

Algoritmos: Un algoritmo es una sucesión finita de pasos organizados de forma ordenada y lógica para lograr un objetivo.

En el presente libro, ese objetivo consiste en orientar y permutar las componentes de la última capa del cubo de Rubik (Permutaciones OLL y PLL respectivamente) para dejarlo en su estado original, una vez se hayan organizado las capas inferior e intermedia. $^{2}$

\subsection{Algoritmos y Estructuras OLL}

Los algoritmos OLL (Orientation of Last Layer: Orientación de la última capa), al igual que los PLL (Permutation of Last Layer: Permutación de la última capa), son una sucesión de movimientos básicos:

2 La organización de las dos inferior e intermedia es tarea del lector, En caso de que no sepa cómo hacerlo recomendamos el método de las dos capas de Fridrich(2005); puede utilizar tutoriales como el que se encuentra la dirección https://blog.kubekings.com/como-hacer-el-cubo-derubik/. Una vez tenga claridad de ese proceso puede proseguir con el estudio de este libro. 


$$
\text { p: } \mathbf{A}_{1} \mathbf{A}_{2} \mathbf{A}_{3} \ldots \mathbf{A}_{\mathbf{n}} \quad \mathrm{A}_{\mathrm{i}}=\mathrm{U}, \mathrm{U}^{\prime}, \mathrm{D}, \mathrm{D} \text {, etc. }
$$

que se ejecutan esencialmente una vez organizadas las capas inferiores e intermedia del cubo.

Los OLL tienen como tarea orientar todo el cubo; una pieza bien orientada es aquella que, al ser llevada a su posición original por rotación, si es necesario, se observa que es la misma que cuando tenemos el cubo en su estado original. Esencialmente son 57 las configuraciones asociadas a estas orientaciones y serán tema de estudio en este libro.

Los conceptos de esta sección no se van a ilustrar ahora, pero se espera que se entiendan a partir del estudio de los OLL que se iniciara desde la próxima sección

\subsubsection{Inverso de un OLL}

Dado un algoritmo OLL

$$
\text { p: } \mathbf{A}_{1} \mathbf{A}_{2} \mathbf{A}_{3} \ldots \mathbf{A}_{\mathrm{n}} \text {. }
$$

su inverso notado p' se define como:

$$
p^{\prime}: A_{n}^{\prime} A_{n-1}^{\prime} A_{n-2}, \ldots A_{2}^{\prime} A_{1}^{\prime}
$$

en otras palabras, se recorre el algoritmo $\mathrm{p}$ en sentido inverso y con los inversos de c/u de los movimientos que lo constituyen.

Observación: Con $\mathbf{p}^{\mathbf{s}}$ se consigue una configuración OLL simétrica a la obtenida de $\mathbf{p}$ con respecto a los lados ID (Izquierdo y derecha). Algo más, $\mathbf{p}^{\mathbf{s}}$ es otro OLL.

Observación: Con p’ se consigue que el cubo vuelva a su estado inmediatamente anterior a la ejecución de p. Algo más, p’ es otro OLL.

\subsubsection{Simétrico de un OLL}

Dado un algoritmo OLL

$$
\text { p: } A_{1} A_{2} A_{3} \ldots A_{n}
$$

su simétrico notado $\mathbf{p}^{\mathrm{S}}$ se define como

$$
p^{s}: A_{1}{ }^{s} A_{2}{ }^{s} A_{3}{ }^{s} \ldots A_{n-1}{ }^{s} A_{n}{ }^{s} \text {, }
$$


En otras palabras, se recorre el algoritmo p en su mismo sentido, pero con los simétricos de c/u de los movimientos que lo constituyen.

\subsubsection{Configuración OLL}

Es la disposición de las componentes de la capa superior (posición y existencia o no de torsión) apenas hayamos organizado las capas inferior e intermedia o cuando se aplica un OLL. En este caso el OLL se denomina un constructor de la configuración.

Cada configuración OLL proviene de un OLL y a su vez da lugar a un OLL; es decir, si tenemos una configuración OLL a su lado hay un constructor que la genera.

\subsubsection{Estructura OLL.}

En todo OLL se distinguen fundamentalmente tres partes: Configuración (F), un constructor y un deconstructor de F.

\subsubsection{1 $\mathrm{c}(\mathrm{F})$ : constructor de la configuración $F$}

Es un OLL cuya ejecución da lugar a F. Normalmente se dice que " $c(F)$ configura la cara superior" y su configuración es F.

\subsubsection{2 $\mathrm{d}(\mathrm{F})$ : deconstructor de la configuración $\mathrm{F}$}

Es un OLL cuya ejecución da lugar al cubo de Rubik totalmente orientado notado como $\mathbf{C}_{\mathrm{I}}$; esto es, ninguna de sus componentes tiene torsión (por eso da la ilusión de que "por fin organizamos el cubo"). Su tarea es pues "orientar bien las componentes que tienen torsión"; y por ello se recupera el color de la cara superior en todas sus componentes, quedando obviamente esta de un solo color. De ese modo, " $\mathrm{d}(\mathrm{F})$ destruye la configuración $\mathrm{F}$ de la cara superior dada por $\mathrm{c}(\mathrm{F})$ "

$$
\mathrm{c}(\mathrm{F}) \longrightarrow \mathrm{F} \longrightarrow \mathrm{d}(\mathrm{F})
$$




\subsubsection{Inversa de una configuración OLL}

Sea C una configuración OLL. Su inversa $C^{\prime}$ es otra configuración OLL cuyo constructor es el deconstructor de $\mathrm{C}, \mathrm{y}$ su deconstructor es el constructor de C. De otra manera, si Ces la configuración generada por el OLL $\mathbf{p}, \mathrm{C}^{\prime}$ es la configuración generada por su inverso OLL p'.

\subsection{Nuestro primer OLL: La $\mathrm{T}_{1}$}

\subsubsection{Configuración, constructor y deconstructor de " $\mathrm{T}_{1}$ ”}

$\mathbf{T}_{1}$ :

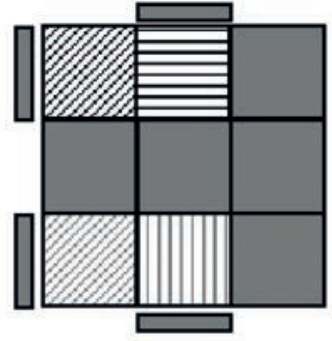

$c\left(T_{1}\right): \quad F\left(U R U^{\prime} R^{\prime}\right) F^{\prime}$

$d\left(T_{1}\right): \quad F\left(R \cup R^{\prime} U^{\prime}\right) F^{\prime}$

A partir del cubo en su estado original con la cara amarilla como cara superior y la cara roja como frontal ${ }^{3}$, al desarrollar el algoritmo $\mathrm{c}\left(\mathrm{T}_{1}\right)$ obtenemos la configuración $\mathrm{T}_{1}$
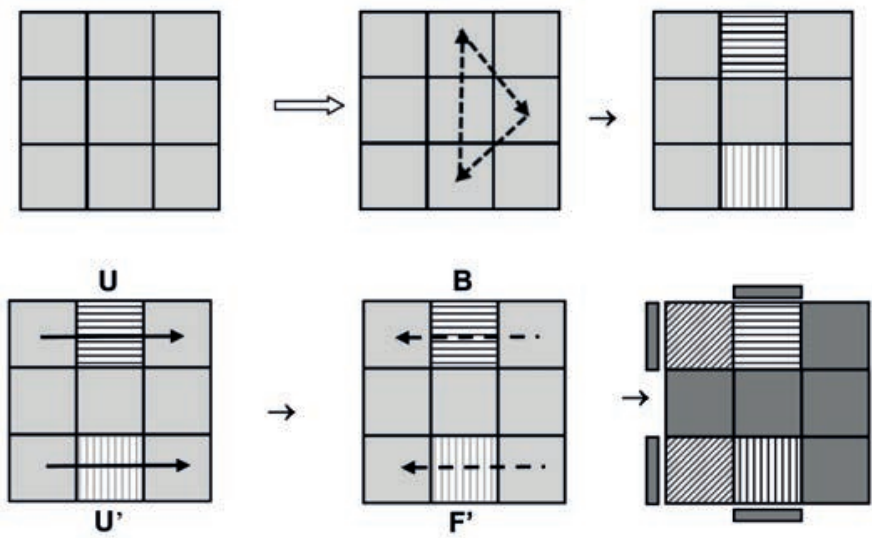

3 El lector puede escoger otras caras como superior e inferior. Y desde luego hacer eso es practicar bien los algoritmos para luego memorizarlos. 


\section{Comentarios sobre $\mathrm{T}_{1}$ :}

- La T está formada por los stickers que son del color de la cara superior y se encuentran en las componentes que han quedado "bien orientadas" una vez ejecutado el algoritmo $\mathrm{c}\left(\mathrm{T}_{1}\right)$.

- Nótese que $\mathrm{d}\left(\mathrm{T}_{1}\right)$ se construye devolviéndonos por la ruta de $c\left(\mathrm{~T}_{1}\right)$ considerando en cada paso el inverso del básico usado en dicha ruta.

- Por lo expuesto en la sección 1.2, los cuadros resaltados externamente en la configuración indican que esas componentes tienen torsión.

- Tal es así como en la cara superior:

- Sus vértices izquierdos presentan torsión izquierda;

- Su arista inferior presenta torsión frontal en tanto que su arista superior tiene torsión trasera.

- Las componentes restantes están bien orientadas (La T se visualiza en color gris).

\subsubsection{Dinámica del constructor de $\mathrm{T}_{1}, \mathrm{c}\left(\mathrm{T}_{1}\right)$}

\subsubsection{Acción de $c\left(\mathrm{~T}_{1}\right)$ sobre sus aristas iniciales.}

$\mathrm{C}\left(\mathrm{T}_{1}\right)$ realiza una permutación en sentido horario de tres de las aristas distintas de la izquierda (permanece invariante), provocando permutaciones en los stickers de algunas de estas.

De modo preciso:

- La arista superior pasa a ser la arista derecha;

- La arista derecha pasa a ser la inferior y sus stickers permutan;

- La arista inferior pasa a ser la superior y sus stickers permutan. 


\subsubsection{Acción de $\mathrm{c}\left(\mathrm{T}_{1}\right)$ sobre sus vértices iniciales}

Los vértices superiores permutan entre sí; lo mismo podemos decir de los vértices inferiores.

Precisando tenemos:

- El vértice inferior izquierdo pasa a ser el vértice inferior derecho mediante un movimiento U'; y viceversa, el vértice inferior derecho pasa a ser el vértice inferior izquierdo, pero con un movimiento F'.

- El vértice superior izquierdo pasa a ser el vértice superior derecho mediante un movimiento $\mathrm{U}$; y viceversa, el vértice superior derecho pasa a ser el vértice superior izquierdo, pero con un movimiento $B$.

Comprobaciones: Una vez realizada la configuración $\mathrm{T}_{1}$ :

- Ejecute el movimiento U, ¿Observa el vértice inferior izquierdo en su posición inicial? Hecha la observación, ejecute de nuevo el movimiento U'.

- Ejecute el movimiento $F$, ¿Observa el vértice inferior derecho en su posición inicial? Hecha la observación ejecute de nuevo el movimiento F'.

- Ejecute el movimiento U', ¿Observa el vértice superior izquierdo en su posición inicial? Hecha la observación ejecute de nuevo el movimiento $\mathrm{U}$.

- Ejecute el movimiento B', ¿Observa el vértice superior derecho en su posición inicial? Hecha la observación ejecute de nuevo el movimiento $B$.

\subsubsection{Descripción de la acción de $c\left(T_{1}\right)$ sobre sus vértices iniciales usando torsiones de tres giros y $\mathrm{U}$ \\ Otra forma de expresar esas permutaciones en los vértices, no muy práctica pero interesante, es mediante torsiones positivas o negativas de 3 giros para un vértice.}




\subsection{Una torsión horaria de 3 giros para un vértice:}

Es aquella donde al colocar en su posición inicial el vértice a examinar y luego al girar el cubo hasta dejar tal vértice como vértice inferior derecho de la capa superior se observa que sus tres stickers permutan en "sentido horario";

Esto es:

Su sticker superior inicial (en el cubo en su estado original) está en la cara derecha; su sticker inicial derecho está en la cara frontal; y su sticker frontal inicial está en la cara superior.

\subsection{Una torsión antihoraria de 3 giros para un vértice:}

Es aquella donde al colocar en su posición inicial el vértice a examinar y luego al girar el cubo hasta dejar tal vértice como vértice inferior derecho de la capa superior se observa que sus tres stickers permutan en "sentido antihorario"; Esto es:

Su sticker superior inicial (en el cubo en su estado original) está en la cara frontal; su sticker inicial frontal está en la cara derecha; y su sticker derecho inicial está en la cara superior.

Usando esos dos conceptos podemos plantear que las permutaciones que se dan en los vértices de la capa superior mediante la configuración $\mathrm{T}_{1}$ son las siguientes:

- El vértice inferior izquierdo inicial pasa a ser el inferior derecho mediante un movimiento $U^{\prime}$; en tanto que el vértice inferior derecho inicial sufre una torsión "antihoraria" de tres giros y pasa a ser el inferior izquierdo con un movimiento $\mathrm{U}$.

- El vértice superior izquierdo inicial pasa a ser el superior derecho mediante un movimiento $\mathrm{U}$; en tanto que el vértice superior derecho inicial sufre una torsión "horaria" de tres giros y pasa a ser el superior izquierdo con un movimiento U'. 
Comentario: Una vez observado sobre todo lo inherente a las torsiones de tres giros ubique la configuración $T_{1}$ en la posición descrita en su configuración inicial y luego use $\mathrm{d}\left(\mathrm{T}_{1}\right)$

$1.7 \mathrm{P}_{1}$ : El inverso de $\mathrm{T}_{1}$ o La Primera " $\mathrm{P}$ "

1.7.1 Configuración, constructor y deconstructor de " $\mathrm{P}$ "

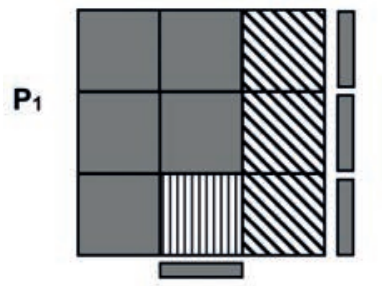

$$
\begin{array}{ll}
c\left(P_{1}\right)=c\left(T_{1}{ }^{\prime}\right)=d\left(T_{1}\right): & F\left(R \cup R^{\prime} U^{\prime}\right) F^{\prime} \\
d\left(P_{1}\right)=d\left(T_{1}{ }^{\prime}\right)=c\left(T_{1}\right): & F\left(U R U^{\prime} R^{\prime}\right) F^{\prime}
\end{array}
$$

\subsubsection{Comentarios sobre $\mathrm{P}_{1}$}

- A partir del cubo en su estado original con la cara amarilla como cara superior y la cara roja como cara frontal, al desarrollar el algoritmo $\mathrm{c}\left(\mathrm{P}_{1}\right)$ obtenemos la configuración $\mathrm{P}_{1}$.

- La "P" está formada por los stickers que son del color de la cara superior y se encuentran en las componentes que han quedado "bien orientadas" una vez ejecutado el algoritmo $\mathrm{c}\left(\mathrm{P}_{1}\right)$.

- El constructor de $\mathrm{P}_{1}$ se construye devolviéndonos por la ruta de $\mathrm{c}\left(\mathrm{T}_{1}\right)$ considerando en cada paso el inverso del básico usado en dicha ruta

$$
\begin{aligned}
& c\left(T_{1}\right): \quad F\left(R \text { U R' U') } F^{\prime}\right. \\
& c\left(P_{1}\right)=c\left(T_{1}\right) \leftarrow^{\prime}: F\left(U R U^{\prime} R^{\prime}\right) F^{\prime}
\end{aligned}
$$

- De modo análogo, el deconstructor de $\mathrm{P}_{1}$ se construye devolviéndonos por la ruta de $\mathrm{d}\left(\mathrm{T}_{1}\right)$ considerando en cada paso el inverso del básico usado en dicha ruta

- $\mathrm{d}\left(\mathrm{P}_{1}\right)$ se construye devolviéndonos por la ruta de $\mathrm{c}\left(\mathrm{P}_{1}\right)$ considerando en cada paso el inverso del básico usado en dicha ruta. 
- En la cara superior:

- Todas las componentes de su lado derecho tienen torsión derecha;

- Su arista inferior presenta torsión frontal.

- Las componentes restantes están bien orientadas.

1.7.3 Dinámica del constructor de $\mathrm{P}_{1}, \mathrm{c}\left(\mathrm{P}_{1}\right)=\mathrm{d}\left(\mathrm{T}_{1}\right)$
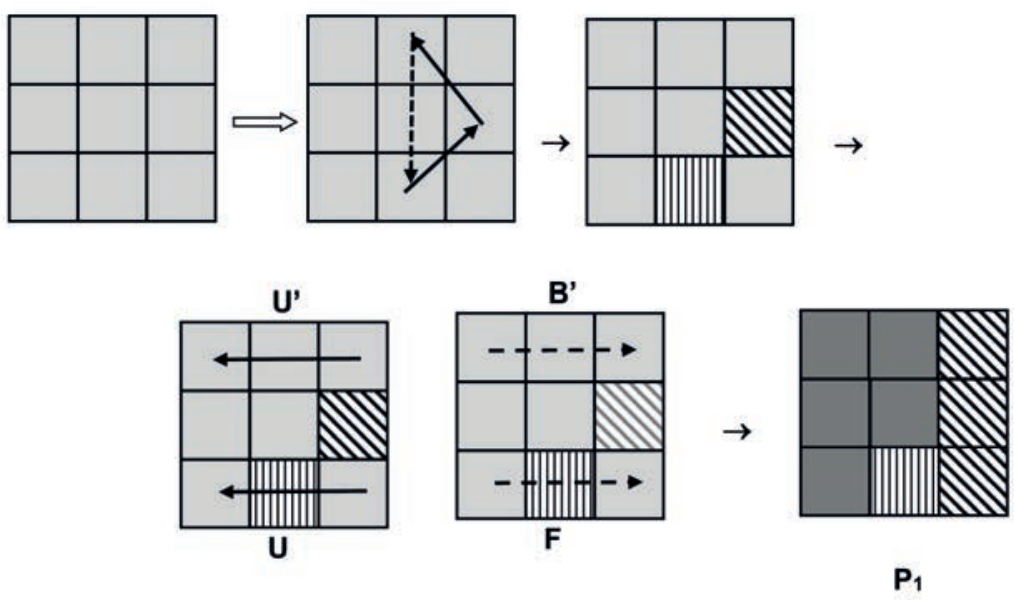

$c\left(P_{1}\right)$ realiza lo de $c\left(T_{1}\right)$ pero en sentido opuesto tanto en las aristas como en los vértices.

\subsubsection{Acción de $c\left(P_{1}\right)$ sobre sus aristas iniciales: $c\left(P_{1}\right)$}

Realiza una permutación en sentido antihorario de tres de las aristas distintas de la izquierda (permanece invariante), provocando permutaciones en los stickers de algunas de estas. De modo preciso:

- La arista superior pasa a ser la inferior y sus stickers permutan;

- La arista inferior pasa a ser la derecha y sus stickers permutan;

- La arista derecha pasa a ser la superior. 


\subsubsection{Acción de $\mathrm{c}\left(\mathrm{P}_{1}\right)$ sobre sus vértices iniciales:}

Los vértices superiores permutan entre sí; lo mismo podemos decir de los vértices inferiores. Precisando tenemos:

- El vértice inferior derecho pasa a ser el vértice inferior izquierdo mediante un movimiento $U$; y viceversa, el vértice inferior izquierdo pasa a ser el vértice inferior derecho, pero con un movimiento $\mathrm{F}$.

- El vértice superior derecho pasa a ser el vértice superior izquierdo mediante un movimiento U'; y viceversa, el vértice superior izquierdo pasa a ser el vértice superior derecho, pero con un movimiento B'.

Comprobaciones: Una vez realizada la configuración $\mathrm{P}_{1}$ :

- Ejecute el movimiento U', ¿Observa el vértice inferior derecho en su posición inicial? Hecha la observación ejecute de nuevo el movimiento $\mathrm{U}$.

- Ejecute el movimiento F’ ¿Observa el vértice inferior izquierdo en su posición inicial? Hecha la observación ejecute de nuevo el movimiento $\mathrm{F}$.

- Ejecute el movimiento U, ¿Observa el vértice superior derecho en su posición inicial? Hecha la observación ejecute de nuevo el movimiento U'.

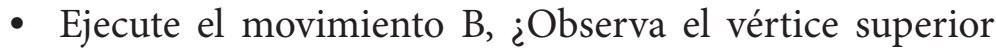
izquierdo en su posición inicial? Hecha la observación ejecute de nuevo el movimiento B'.

\subsubsection{Descripción de la acción de $c\left(P_{1}\right)$ sobre sus vértices iniciales usando torsiones de tres giros y $\mathrm{U}$}

- El vértice inferior derecho inicial pasa a ser el vértice inferior izquierdo mediante un movimiento U; en tanto que el vértice inferior izquierdo inicial sufre una torsión "horaria" de tres giros y pasa a ser el vértice inferior derecho con un movimiento U'. 
- El vértice superior derecho inicial pasa a ser el superior izquierdo mediante un movimiento U'; en tanto que el vértice superior izquierdo inicial sufre una torsión "antihoraria" de tres giros y pasa a ser el vértice superior derecho con un movimiento $\mathrm{U}$.

Comentario: Una vez observado sobre todo lo inherente a las torsiones de tres giros ubique la configuración $\mathrm{P}_{1}$ en la posición descrita en su configuración inicial y luego use $\mathrm{d}\left(\mathrm{P}_{1}\right)$

\section{$1.8 \mathrm{~T}_{1}^{\mathrm{s}}$ : La Simétrica de $\mathrm{T}_{1}$}

1.8.1 Configuración, constructor y de constructor de $\mathrm{T}_{1}^{\mathrm{S}}$

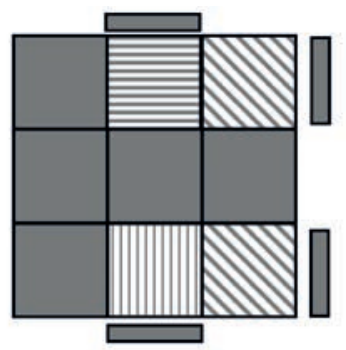

$$
\begin{array}{ll}
c\left(T_{1} s\right)=c\left(T_{1}\right)^{s:} & F^{\prime}\left(U^{\prime} L^{\prime} U L\right) F \\
d\left(T_{1} s\right)=d\left(T_{1}\right)^{s}: & F^{\prime}\left(L^{\prime} U^{\prime} L U\right) F
\end{array}
$$

1.8.2 Comentarios sobre $\mathrm{T}_{1}^{\mathrm{s}}$

- Su constructor se diseña a partir del constructor de $\mathrm{T}_{1}$, pero cambiando cada eslabón de la cadena por su simétrico:

$$
\begin{aligned}
& \mathrm{c}\left(\mathrm{T}_{1}{ }^{\mathrm{s}}\right)=\mathrm{c}\left(\mathrm{T}_{1}\right)^{\mathrm{s}} \\
& \text { ¿Por qué? } \\
& \mathrm{c}\left(\mathrm{T}_{1}\right)=\mathrm{F}\left(\mathrm{U} \mathrm{R} \mathrm{U}^{\prime} \mathrm{R}\right. \text { ') F', de 1.6.1 } \\
& \mathrm{c}\left(\mathrm{T}_{1}\right)^{\mathrm{s}}=\mathrm{F}^{\prime}\left(\mathrm{U}^{\prime} \mathrm{L}\right. \text { ' U L) F, de 1.5.2. y 1.3.1.2 } \\
& =\mathrm{c}\left(\mathrm{T}_{1}{ }^{\mathrm{s}}\right)
\end{aligned}
$$

- De modo análogo, $\mathrm{d}\left(\mathrm{T}_{1}^{\mathrm{S}}\right)=\mathrm{d}\left(\left(\mathrm{T}_{1}\right)^{\mathrm{S}}\right.$

- En la cara superior:

- Sus vértices derechos presentan torsión derecha; 
- Su arista inferior presenta torsión frontal en tanto que su arista superior tiene torsión trasera.

- Las componentes restantes están bien orientadas.

- $\mathrm{T}_{1}$ y su simétrica son configuraciones equivalentes ${ }^{4}: \mathrm{T}_{1} \sim$ $\mathrm{T}_{1}{ }_{1}^{\mathrm{S}}$. Observamos que la configuración $\mathrm{T}_{1}{ }^{\mathrm{S}}$ se obtiene de la configuración $\mathrm{T}_{1}$ al rotarse la capa superior $180^{\circ}$

$$
\mathrm{T}_{1}^{\mathrm{S}}=\mathrm{T}_{1} \mathrm{U} 2 \text {. }
$$

Dos configuraciones OLL $C_{1}$ y $C_{2}$ son equivalentes, $C_{1} \sim C_{2}$, si y sólo si una de ellas se obtiene de la otra mediante giros de la capa superior. Las configuraciones que damos inicialmente para cada OLL $p$ son las referenciales para comparar (canónicas) y se simbolizan como $\widehat{\mathrm{C}_{\mathrm{p}}}$; aunque en el momento simplemente las notaremos $C_{p}$.

1.8.3 Dinámica del constructor de $\mathrm{T}_{1}^{\mathrm{S}}$
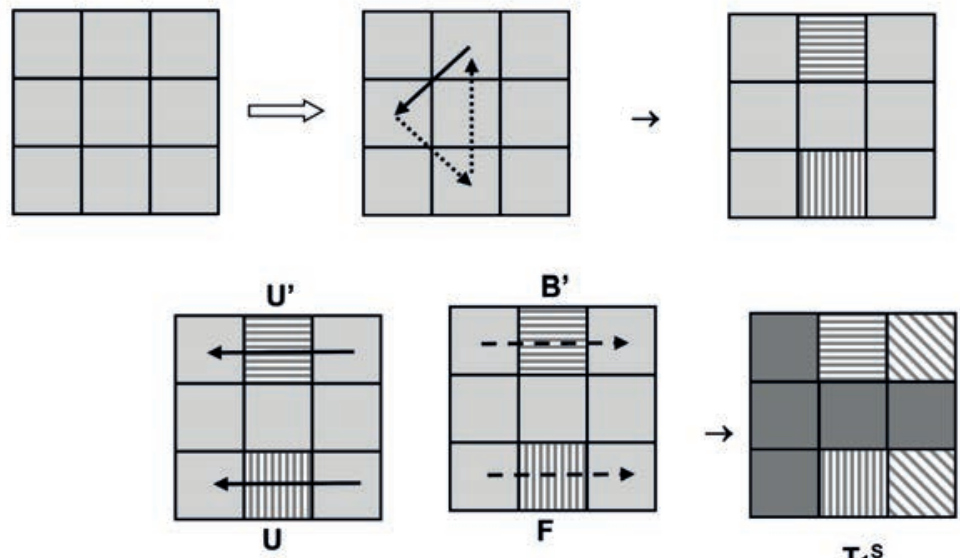

$$
T_{1} s
$$

$\mathrm{c}\left(\mathrm{T}_{1}^{S}\right)$ realiza lo de $\mathrm{c}\left(\mathrm{T}_{1}\right)$ pero de modo simétrico con respecto a los lados izquierdo y derecho de la capa superior.

\subsubsection{Acción de $\mathrm{c}\left(\mathrm{T}_{1}^{\mathrm{S}}\right)$ sobre sus aristas iniciales}

$c\left(\mathrm{~T}_{1}^{\mathrm{S}}\right)$ realiza una permutación en sentido antihorario de tres de las aristas distintas de la derecha (permanece invariante), provocando permutaciones en los stickers de algunas de estas. De modo preciso:

4 Por ello esta configuración simétrica no se incluye en el listado de los 57 clásicos OLL; pero resulta muy interesante aprender a manejarlo de modo adecuado y su inversa produce una nueva OLL. 
- La arista superior pasa a ser la arista izquierda;

- La arista izquierda pasa a ser la inferior y sus stickers permutan;

- La arista inferior pasa a ser la superior y sus stickers permutan.

\subsubsection{Acción de $\mathrm{c}\left(\mathrm{T}_{1}{ }^{S}\right)$ sobre sus vértices iniciales}

Los vértices superiores permutan entre sí; lo mismo podemos decir de los vértices inferiores. Todo ello de modo simétrico a las permutaciones realizadas con el $c\left(\mathrm{~T}_{1}\right)$, igual a lo que realiza $\mathrm{c}\left(\mathrm{P}_{1}\right)$ con los vértices.Precisando tenemos:

- El vértice inferior derecho inicial pasa a ser el vértice inferior izquierdo mediante un movimiento $U$; $y$ viceversa, el vértice inferior izquierdo inicial pasa a ser el vértice inferior derecho, pero con un movimiento $\mathrm{F}$.

- El vértice superior derecho inicial pasa a ser el vértice superior izquierdo mediante un movimiento $U^{\prime} ; \mathrm{y}$ viceversa, el vértice superior izquierdo inicial pasa a ser el vértice superior derecho, pero con un movimiento B'.

\subsubsection{Descripción de la acción $\operatorname{de~} c\left(T_{1}{ }^{S}\right)$ sobre sus vértices iniciales usando torsiones de tres giros y $\mathrm{U}$}

- El vértice inferior derecho inicial pasa a ser el vértice inferior izquierdo mediante un movimiento $\mathrm{U}$; en tanto que el vértice inferior izquierdo inicial sufre una torsión "horaria" de tres giros y pasa a ser el vértice inferior derecho con un movimiento U'.

- El vértice superior derecho inicial pasa a ser el vértice superior izquierdo mediante un movimiento U'; en tanto que el vértice superior izquierdo inicial sufre una torsión "antihoraria" de tres giros y pasa a ser el superior derecho con un movimiento U'. 
Comentario: Una vez observado sobre todo lo inherente a las torsiones de tres giros ubique la configuración $\mathrm{T}_{1}{ }^{S}$ en la posición descrita en su configuración inicial y luego use $\mathrm{d}\left(\mathrm{T}_{1} \mathrm{~S}\right)$.

$1.9 \mathrm{q}_{1}$ : El inverso del simétrico de $\mathrm{T}_{1} \mathrm{o}$ La Primera "q"

1.9.1 Configuración, constructor y deconstructor de $\mathrm{q}_{1}$

$\mathbf{q}_{1}$

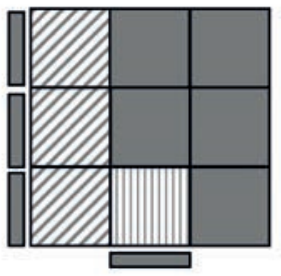

$c\left(q_{1}\right)=c\left(\left(T_{1}^{s}\right)\right)^{\prime}=d\left(T_{1} s^{\prime}\right): F^{\prime}\left(L^{\prime} U^{\prime} L U\right) F$ $d\left(q_{1}\right)=d\left(\left(T_{1}^{S}\right)\right)^{\prime}=c\left(T_{1}^{S}\right): F^{\prime}\left(U^{\prime} L^{\prime} U L\right) F$

1.9.2 Comentarios sobre $\mathrm{q}_{1}$

- Su constructor es el deconstructor de $\mathrm{T}_{1}^{\mathrm{S}}$; Su deconstructor es el constructor de $\mathrm{T}_{1}^{\mathrm{S}}$

- Su constructor es el simétrico del constructor de $\mathrm{T}_{1}$ ':

$\mathrm{c}\left(\left(\mathrm{T}_{1}^{\mathrm{S}}\right)\right)^{\prime}=\mathrm{c}\left(\left(\mathrm{T}_{1}^{\prime}\right)\right)^{\mathrm{S}}$

¿Por qué?

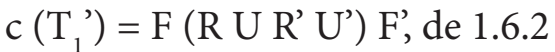

$c\left(\mathrm{~T}_{1}^{\prime}\right)^{\mathrm{S}}=\mathrm{F}^{\prime}\left(\mathrm{L}^{\prime} \mathrm{U}^{\prime} \mathrm{L} \mathrm{U}\right) \mathrm{F}=\mathrm{c}\left(\mathrm{T}_{1}^{\mathrm{S}}\right)^{\prime}$

- De modo análogo, $\mathrm{d}\left(\mathrm{T}_{1}^{\mathrm{S}}\right)^{\prime}=\mathrm{d}\left(\mathrm{T}_{1}{ }^{\prime}\right)^{\mathrm{S}}$

Comentario algebraico. Por esto y lo anterior suele decirse:

"El simétrico del inverso es el inverso del simétrico"

Si usted compara esta configuración con la dada en

1.7.1 notará que las dos son simétricas (la una es simétrica de la otra), que es precisamente lo que expresa la anterior afirmación. 
- En la cara superior:

- Todas las componentes de su lado izquierdo tienen torsión derecha;

- Su arista inferior presenta torsión frontal.

- Las componentes restantes están bien orientadas.

1.9.3 Dinámica del constructor de $\mathrm{q}_{1}, \mathrm{c}\left(\mathrm{q}_{1}\right)=\mathrm{d}\left(\mathrm{T}_{1}{ }^{\mathrm{S}}\right)$
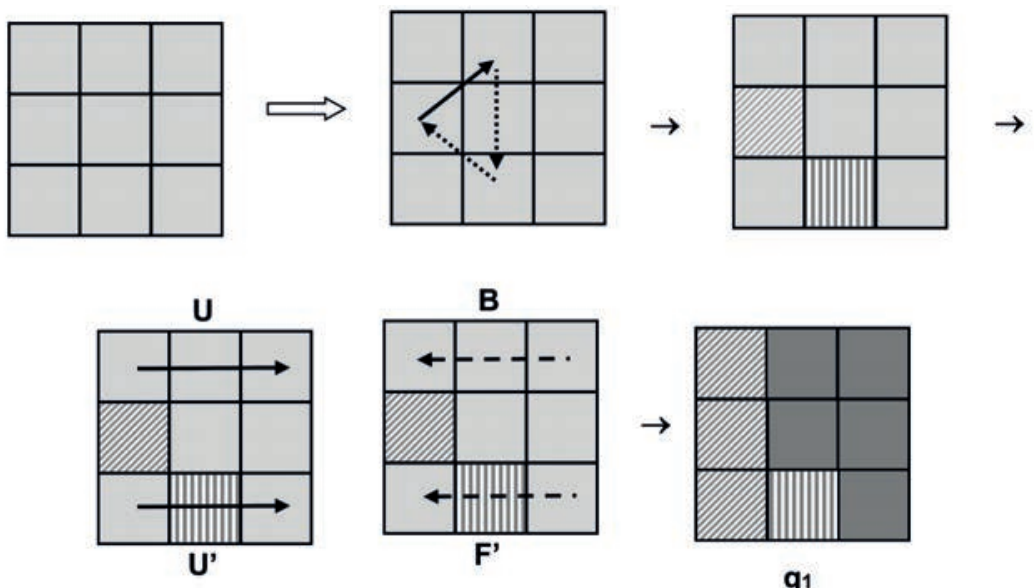

q1

$c\left(\mathrm{q}_{1}\right)$ realiza lo mismo que $\mathrm{c}\left(\mathrm{P}_{1}\right)$ pero en sentido simétrico tanto en las aristas como en los vértices

\subsubsection{Acción de $\mathrm{c}\left(\mathrm{q}_{1}\right)$ sobre sus aristas iniciales:}

$c\left(q_{1}\right)$ realiza una permutación en sentido horario de tres de las aristas distintas de la derecha (permanece invariante), provocando permutaciones en los stickers de algunas de estas. De modo preciso:

- La arista superior pasa a ser la inferior y sus stickers permutan;

- La arista inferior pasa a ser la izquierda y sus stickers permutan;

- La arista izquierda pasa a ser la superior. 


\subsubsection{Acción de $\mathrm{c}\left(\mathrm{q}_{1}\right)$ sobre sus vértices iniciales}

Los vértices superiores permutan entre sí; lo mismo podemos decir de los vértices inferiores. Todo ello de modo simétrico a las permutaciones realizadas con el $\mathrm{c}\left(\mathrm{P}_{1}\right)$, igual a lo que realiza $\mathrm{c}\left(\mathrm{T}_{1}\right)$ con los vértices:

- El vértice inferior izquierdo pasa a ser el vértice inferior derecho mediante un movimiento U'; y viceversa, el vértice inferior derecho pasa a ser el vértice inferior izquierdo, pero con un movimiento F'.

- El vértice superior izquierdo pasa a ser el vértice superior derecho mediante un movimiento $\mathrm{U}$; y viceversa, el vértice superior derecho pasa a ser el vértice superior izquierdo, pero con un movimiento B.

\subsubsection{Descripción de la acción $\operatorname{de~} c\left(q_{1}\right)$ sobre sus vértices iniciales usando torsiones de tres giros y $\mathrm{U}$}

- El vértice inferior izquierdo inicial pasa a ser el vértice inferior derecho mediante un movimiento U'; en tanto que el vértice inferior derecho inicial sufre una torsión "antihoraria" de tres giros y pasa a ser el vértice inferior izquierdo con un movimiento U.

- El vértice superior izquierdo inicial pasa a ser el vértice superior derecho mediante un movimiento $\mathrm{U}$; en tanto que el vértice superior derecho inicial sufre una torsión "horaria" de tres giros y pasa a ser el vértice superior izquierdo con un movimiento U’.

Comentario: Una vez observado sobre todo lo inherente a las torsiones de tres giros ubique la configuración $\mathrm{q}_{1}$ en la posición descrita en su configuración inicial y luego use $\mathrm{d}\left(\mathrm{q}_{1}\right)$ 
Síntesis tabular "Canónica” (secciones $1.6 \rightarrow 1.9$ )

Los primeros 4 OLL para practicar y memorizar

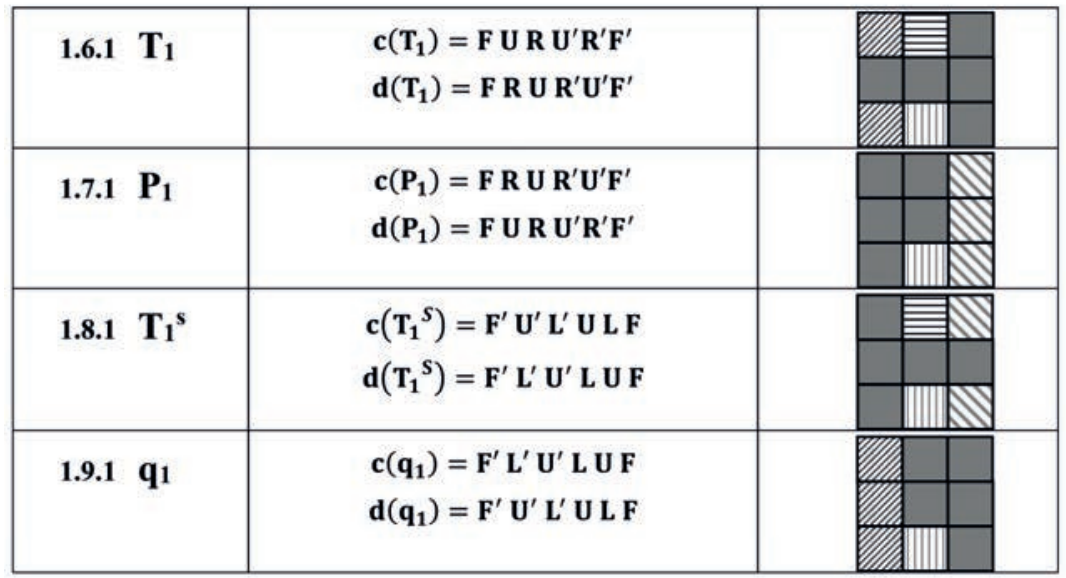

1.10 Observaciones Numéricas Interesantes (Cubo original $3 \times 3 \times 3)$

- Dado que el cubo original $3 \times 3 \times 3$ tiene 8 vértices y 12 aristas, existe 8 (40320) combinaciones para los vértices del cubo, de los cuales 7 pueden ser orientados de forma independiente y la última dependerá de las 7 anteriores, lo cual ofrece $3^{7}(2187)$ posibilidades. 11 aristas pueden ser orientadas independientemente y la última dependerá de las 11 anteriores, con lo cual se obtiene $2^{11}$ (2048) posibilidades En total el número de permutaciones que son posibles en el cubo de Rubik es de:

“43252003274489856 000” es decir, cuarenta y tres trillones, doscientos cincuenta y dos mil tres billones doscientos setenta y cuatro mil cuatrocientos ochenta y nueve millones ochocientos cincuenta y seis mil permutaciones

- La posición correcta de las aristas de la capa inferior (Cruz ampliada inferior) se puede obtener con un máximo de 8 movimientos básicos. 
- Una vez organizadas las aristas de la capa inferior, organizar el resto de la capa inferior y la capa intermedia usando el método del principiante ${ }^{5}$, tiene en cuenta que:

- Cada vértice de la capa inferior, posicionado en la capa superior y en las dos capas de donde proviene distintas a la inferior, se organiza con un mínimo de tres movimientos y un máximo de ocho.

- Cada arista de la capa intermedia, posicionada en la capa superior y en la capa frontal donde se va a ubicar, se organiza con un máximo de nueve movimientos.

- Organizar las aristas de la capa superior usando el método de principiante, tiene en cuenta que:

- Las aristas se posicionan correctamente con mínimo de 7 movimientos.

- Las aristas se orientan con un mínimo de 6 y un máximo de 13 movimientos básicos.

- Organizar los vértices de la capa superior usando el método de principiante, tiene en cuenta que:

- Los vértices se posicionan correctamente con un mínimo de 7 y un máximo de 15 movimientos.

- Los vértices se orientan con un mínimo de 17 y un máximo de 35 movimientos básicos.

Comentario: cabe resaltar que con el método principiante como mínimo se requieren 37 movimientos para organizar correctamente la capa superior. Pero por el contrario usando algoritmos OLL/PLL, como los expuestos en este libro, lo anterior se logra con un máximo de 29 movimientos

5 Para consultar sobre el método principiante se puede consultar: COMO RESOLVER EL CUBO DE RUBIK 3X3 - Tutorial Fácil para principiantes - Español - Xole Rubik. Recuperado de: https://www.youtube.com/watch?v=XKXh-u0RjVk\&t=1311s 


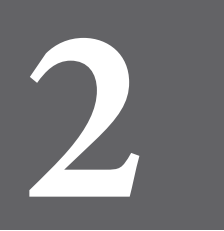

CAPÍTULO DOS 


\section{La Segunda T y Los Primeros siete PLL}

En este segundo capítulo se da a conocer la segunda $T, T_{2}, y$ sus afines (simétrica, inversa e inversa de la simétrica). Además, se desarrollan los primeros siete algoritmos PLL, con los cuales se responde la pregunta ¿Qué hacer una vez orientado totalmente el cubo?, pues los PLL son el último paso para dejar el cubo en su estado original.

2.1 La segunda $\mathrm{T}: \mathrm{T}_{2}$. Su inversa y su simétrica

2.1.1 Configuración, constructor y deconstructor de " $\mathrm{T}_{2}$ "

$T_{2}$

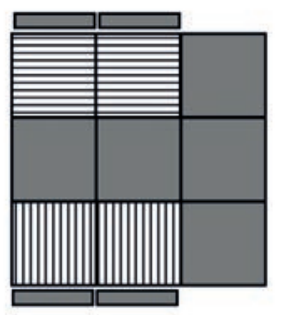

$c\left(T_{2}\right)$ : (F R' F' R) (U R U' R')

$d\left(T_{2}\right)$ : (R U R' U') (R' F R F')

\subsubsection{Comentarios sobre $\mathrm{T}_{2}$}

- A partir del cubo en su estado original con la cara amarilla como cara superior y la cara roja como cara frontal, al desarrollar el algoritmo $\mathrm{c}\left(\mathrm{T}_{2}\right)$ obtenemos la configuración $\mathrm{T}_{2}$.

- La T está formada por los stickers que son del color de la cara superior y se encuentran en las componentes que han quedado "bien orientadas" una vez ejecutado el algoritmo $\mathrm{c}\left(\mathrm{T}_{2}\right)$.

- Nótese que $\mathrm{d}\left(\mathrm{T}_{2}\right)$ se construye devolviéndose por la ruta de $c\left(\mathrm{~T}_{2}\right)$ considerando en cada uno de los pasos el inverso del básico usado en dicha ruta. 
- En la cara superior:

- Su arista inferior y el vértice inferior izquierdo tienen torsión frontal en tanto que su arista superior y el vértice superior izquierdo tienen torsión trasera.

- Las componentes restantes están bien orientadas.

Pregunta: ¿Serán equivalentes las configuraciones $\mathrm{T}_{1}$ y $\mathrm{T}_{2}$ ?, o lo mismo, a partir de $\mathrm{T}_{1}$ y rotaciones de la capa superior $¿ \mathrm{Se}$ podrá obtener la configuración $\mathrm{T}_{2}$ ?

Rta: (para el lector).

\subsubsection{Dinámica del constructor de $T_{2}, c\left(T_{2}\right)$}
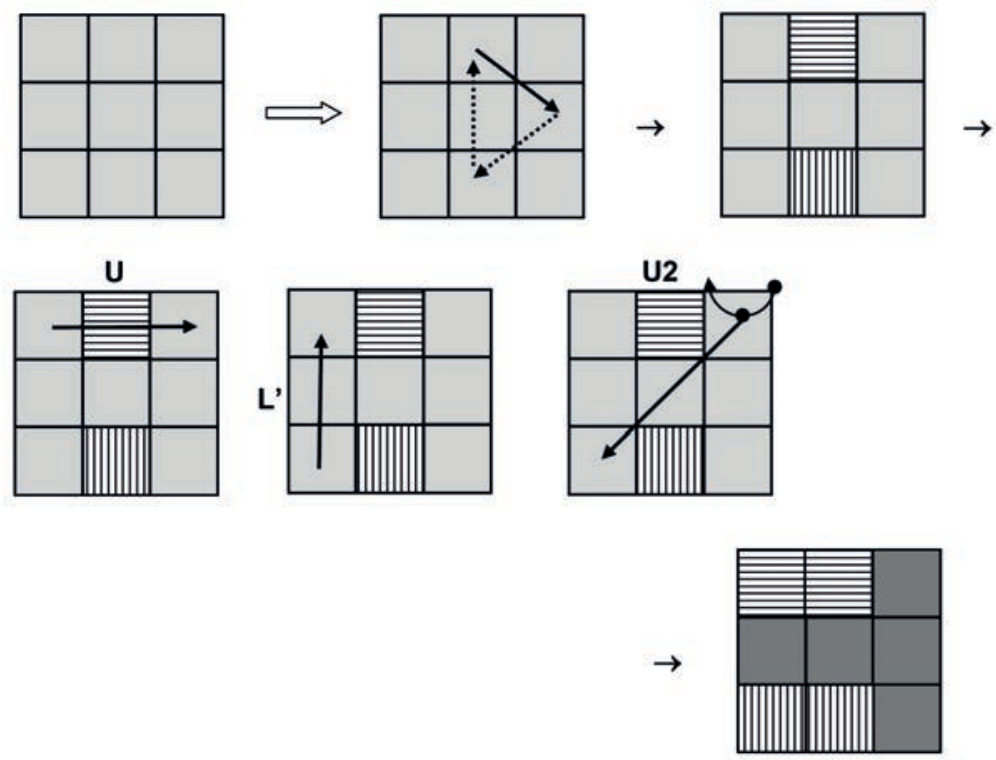

$T_{2}$

\subsubsection{Acción de $\mathrm{c}\left(\mathrm{T}_{2}\right)$ sobre sus aristas iniciales:}

$c\left(\mathrm{~T}_{2}\right)$ realiza, al igual que $\mathbf{c}\left(\mathrm{T}_{1}\right)$, una permutación en sentido horario de tres de las aristas distintas de la izquierda (permanece invariante), provocando permutaciones en los stickers de algunas de estas. 
De modo preciso:

- La arista superior pasa a ser la arista derecha;

- La arista derecha pasa a ser la inferior y sus stickers permutan;

- La arista inferior pasa a ser la superior y sus stickers permutan.

\subsubsection{Acción de $c\left(\mathrm{~T}_{2}\right)$ sobre sus vértices iniciales}

$c\left(\mathrm{~T}_{2}\right)$ realiza una permutación en sentido horario de tres de los vértices distintos del vértice inferior derecho.

De modo preciso:

- El vértice inferior izquierdo inicial pasa a ser el superior izquierdo mediante un movimiento L';

- El vértice superior izquierdo inicial pasa a ser el superior derecho mediante un movimiento U;

- El vértice superior derecho $\left(\mathrm{V}_{\mathrm{SD}}\right)$ inicial recibe una torsión "horaria" de tres giros (1.6.3.3) y luego rota $180^{\circ}$ en la capa superior mediante un movimiento U2. Lo primero significa:

Los tres stickers del vértice superior derecho $\left(\mathrm{V}_{\mathrm{SD}}\right)$ inicial permutan en "sentido horario"

- Su sticker superior inicial pasa a la cara trasera;

- Su sticker trasero inicial pasa la cara derecha; y

- Su sticker derecho inicial pasa a la cara superior.

Desde este momento, por espacio y tiempo, se consignará las configuraciones OLL sin comentario alguno. Se Espera que el lector ya por su propia cuenta realice los análisis pertinentes a las mismas. 
2.2 La Primera Cometa o Cometa ${ }_{1:}$ El inverso de $\mathrm{T}_{2}$

2.2.1 Configuración, constructor y deconstructor de "Cometa"

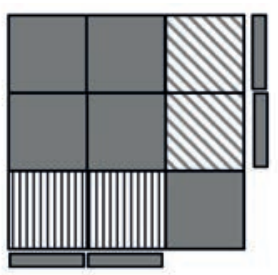

$c($ Cometa 1$)=d\left(T_{2}\right):\left(R \cup R^{\prime} U^{\prime}\right)\left(R^{\prime} F\right.$ R F')

$d\left(C^{\prime}\right.$ ometa 1$)=c\left(T_{2}\right):(F$ R' F' R) (U R U' R')

Cometa 1

2.2.2 Dinámica del constructor de Cometa, $\mathrm{c}\left(\right.$ Cometa $\left._{1}\right)$
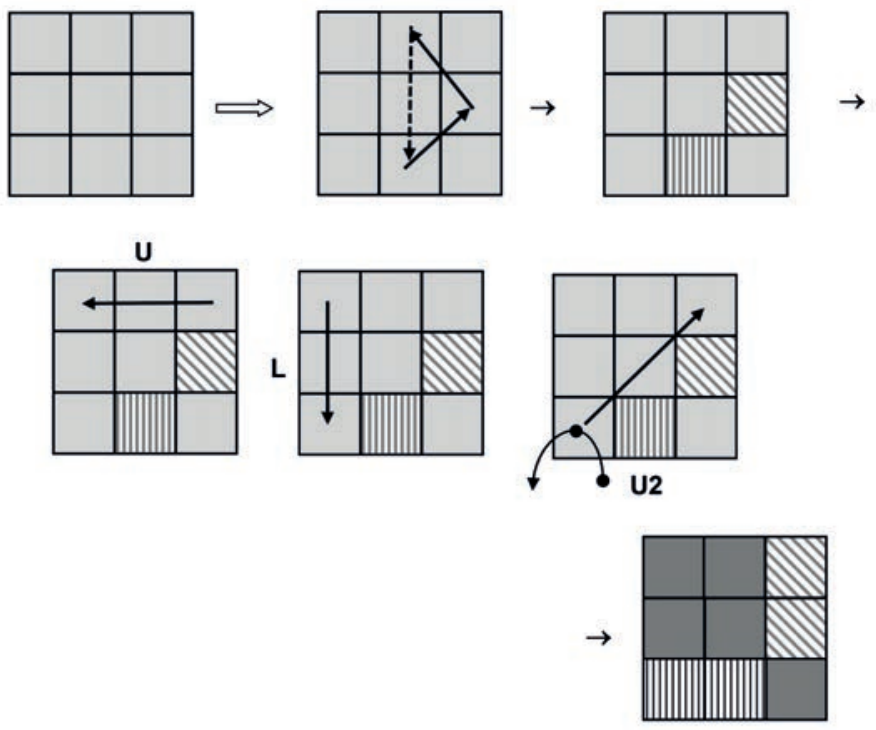

Cometa 1

2.1.3.1 Acción de $c\left(T_{2}\right)$ sobre sus aristas iniciales

$c\left(T_{2}\right)$ realiza, al igual que $c\left(T_{1}\right)$, una permutación en sentido horario de tres de las aristas distintas de la izquierda (permanece invariante), provocando permutaciones en los stickers de algunas de estas. 
De modo preciso:

- La arista superior pasa a ser la arista derecha;

- La arista derecha pasa a ser la inferior y sus stickers permutan;

- La arista inferior pasa a ser la superior y sus stickers permutan.

\subsubsection{Acción de $\mathrm{c}\left(\mathrm{T}_{2}\right)$ sobre sus vértices iniciales}

$c\left(\mathrm{~T}_{2}\right)$ realiza una permutación en sentido horario de tres de los vértices distintos del vértice inferior derecho.

De modo preciso:

- El vértice inferior izquierdo inicial pasa a ser el superior izquierdo mediante un movimiento L';

- El vértice superior izquierdo inicial pasa a ser el superior derecho mediante un movimiento U;

- El vértice superior derecho $\left(\mathrm{V}_{\mathrm{SD}}\right)$ inicial recibe una torsión "horaria" de tres giros (1.6.3.3) y luego rota $180^{\circ}$ en la capa superior mediante un movimiento U2. Lo primero significa:

Los tres stickers del vértice superior derecho $\left(\mathrm{V}_{\mathrm{SD}}\right)$ inicial permutan en "sentido horario"

- Su sticker superior inicial pasa a la cara trasera;

- Su sticker trasero inicial pasa la cara derecha; y

- Su sticker derecho inicial pasa a la cara superior.

Desde este momento, por espacio y tiempo, se consignará las configuraciones OLL sin comentario alguno. Se Espera que el lector ya por su propia cuenta realice los análisis pertinentes a las mismas. 


\subsection{La Primera Cometa o Cometa ${ }_{1:}$ El inverso de $\mathrm{T}_{2}$}

\subsubsection{Configuración, constructor y deconstructor de} "Cometa"

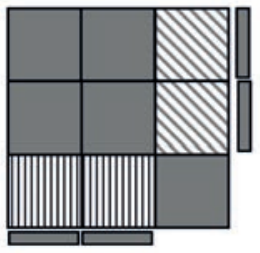

$c\left(C^{\prime} x_{1}\right)=d\left(T_{2}\right):\left(R \cup R^{\prime} U^{\prime}\right)\left(R^{\prime} F\right.$ R F')

Cometa 1

2.2.2 Dinámica del constructor de Cometa, $\mathrm{c}\left(\right.$ Cometa $\left._{1}\right)$
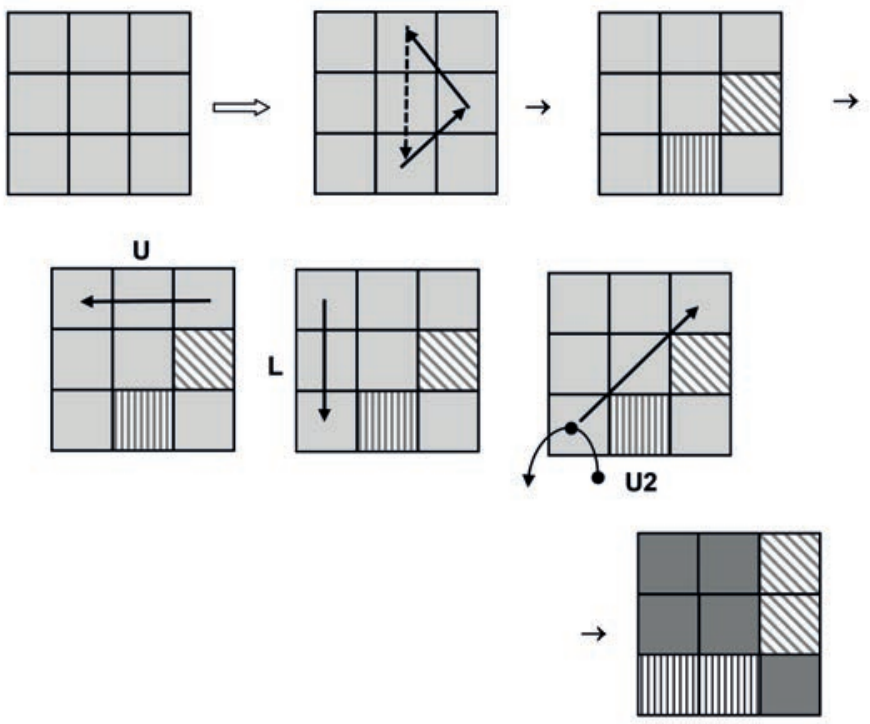

\section{Cometa $_{1}$}

$c\left(\right.$ Cometa $\left._{1}\right)$ realiza lo mismo de $c\left(\mathrm{~T}_{2}\right)$ pero en sentido opuesto, tanto en las aristas como en los vértices.

\subsubsection{Acción de $\mathrm{c}\left(\right.$ Cometa $\left._{1}\right)$ sobre sus aristas iniciales} $c\left(\right.$ Cometa $\left._{1}\right)$, al igual que $c\left(\mathrm{P}_{1}\right)=c\left(\mathrm{~T}_{1}{ }^{\prime}\right)$, realiza una permutación en sentido antihorario de tres de las aristas distintas de la izquierda (permanece invariante), provocando permutaciones en los stickers de algunas de estas. 
De modo preciso:

- La arista superior pasa a ser la inferior y sus stickers permutan;

- La arista inferior pasa a ser la derecha y sus stickers permutan;

- La arista derecha pasa a ser la superior.

\subsubsection{Acción de $\mathrm{c}\left(\right.$ Cometa $\left._{1}\right)$ sobre sus vértices iniciales} $c\left(\right.$ Cometa $\left._{1}\right)$ realiza una permutación en sentido horario de tres de los vértices distintos del vértice inferior derecho.

De modo preciso:

- El vértice superior derecho pasa a ser el superior izquierdo mediante un movimiento U';

- El vértice superior izquierdo pasa a ser el inferior izquierdo mediante un movimiento L;

- El vértice inferior izquierdo $\left(\mathrm{V}_{\mathrm{II}}\right)$ inicial recibe una torsión "antihoraria" de tres giros (1.6.3.3) y luego realiza una rotación de $180^{\circ}$ en la capa superior mediante un movimiento U2.

Lo anterior significa:

Los tres stickers del vértice inferior izquierdo $\mathrm{V}_{\mathrm{II}}$ inicial permutan en "sentido antihorario":

- Su sticker superior inicial pasa a la cara izquierda;

- Su sticker izquierdo inicial pasa la cara frontal;

- Su sticker frontal inicial pasa a la cara superior. 
2.3 La Simétrica de $T_{2}, T_{2}{ }^{\mathrm{S}} \sim \mathrm{T}_{2}$

2.3.1Configuración, constructor y deconstructor de " $\mathrm{T}_{2}^{S "}$

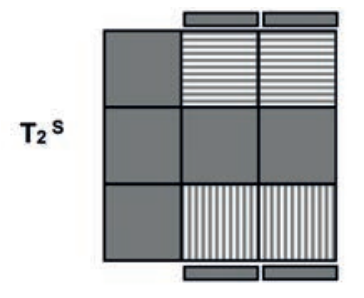

$$
\begin{aligned}
& c\left(T_{2}{ }^{s}\right):\left(F^{\prime} L F L^{\prime}\right)\left(U^{\prime} L^{\prime} U L\right) \\
& \left.d\left(T_{2}{ }^{s}\right):\left(L^{\prime} U^{\prime} L U\right) \text { (LF' } F^{\prime} L^{\prime} F\right) \\
& T_{2} s=T_{2} U 2 \sim T_{2}
\end{aligned}
$$

$c\left(\mathrm{~T}_{2}^{\mathrm{S}}\right)$ realiza lo de $\mathrm{c}\left(\mathrm{T}_{2}\right)$ pero de modo simétrico con respecto a los lados izquierdo y derecho de la capa superior.

\subsubsection{Dinámica del constructor de $\mathrm{T}_{2} \mathrm{~s}$}
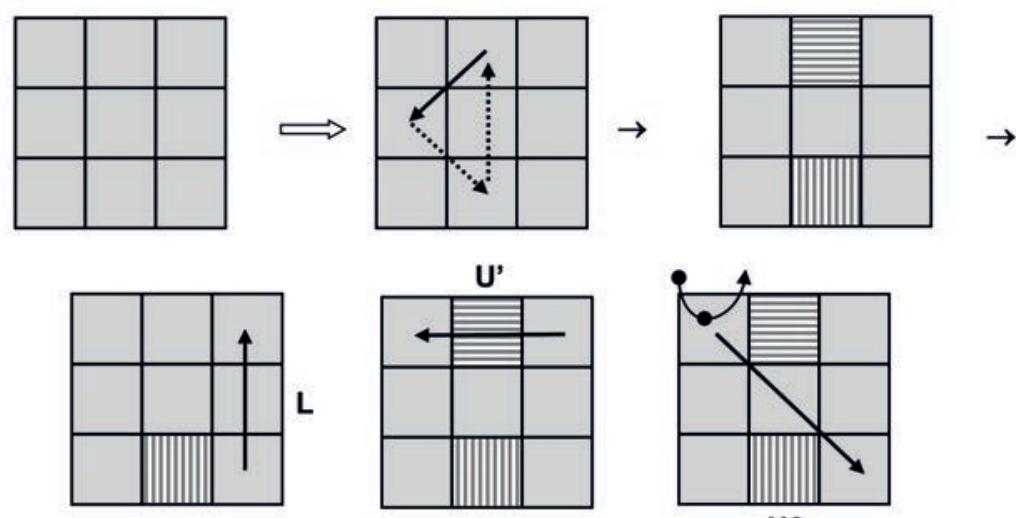

$$
\text { U2 }
$$

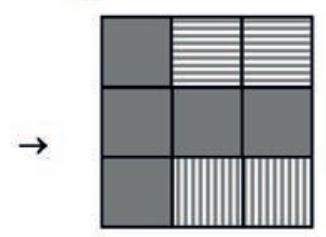

$\mathrm{T}_{2} \mathrm{~S}$

2.3.2.1 Acción de $\mathrm{c}\left(\mathrm{T}_{2}{ }^{\mathrm{s}}\right)$ sobre sus aristas iniciales

$\mathrm{c}\left(\mathrm{T}_{2}^{\mathrm{S}}\right)$ realiza una permutación en sentido antihorario de tres de las aristas distintas de la derecha (permanece invariante), provocando permutaciones en los stickers de algunas de estas. 
De modo preciso:

- La arista superior pasa a ser la arista izquierda;

- La arista izquierda pasa a ser la inferior y sus stickers permutan;

- La arista inferior pasa a ser la superior y sus stickers permutan.

\subsubsection{Acción de $c\left(\mathrm{~T}_{2}{ }^{\mathrm{S}}\right)$ sobre sus vértices iniciales}

$\mathrm{c}\left(\mathrm{T}_{2}{ }^{\mathrm{S}}\right)$ realiza una permutación en sentido antihorario de tres de los vértices distintos del vértice inferior izquierdo.

De modo preciso:

- El vértice inferior derecho inicial pasa a ser el vértice superior derecho mediante un movimiento R;

- El vértice superior derecho inicial pasa a ser el vértice superior izquierdo mediante un movimiento U';

- El vértice superior izquierdo $\left(\mathrm{V}_{\mathrm{SI}}\right)$ inicial recibe una torsión "antihoraria" de tres giros (1.6.3.3) y luego realiza una rotación de $180^{\circ}$ en la capa superior mediante un movimiento U2.

Lo anterior significa:

Los tres stickers del Vértice superior izquierdo $\left(\mathrm{V}_{\mathrm{II}}\right)$ inicial permutan en "sentido antihorario"

- Su sticker superior inicial pasa a la cara trasera;

- Su sticker trasero inicial pasa la cara izquierda; y

- Su sticker izquierdo inicial pasa a la cara superior. 
2.4 Cometa ${ }_{1}{ }^{\mathrm{S}}:$ EL inverso del simétrico de $\mathrm{T}_{2}$

2.4.1 Configuración, constructor y deconstructor de $\left(\mathrm{T}_{2}^{\mathrm{S}}\right)^{\prime}$

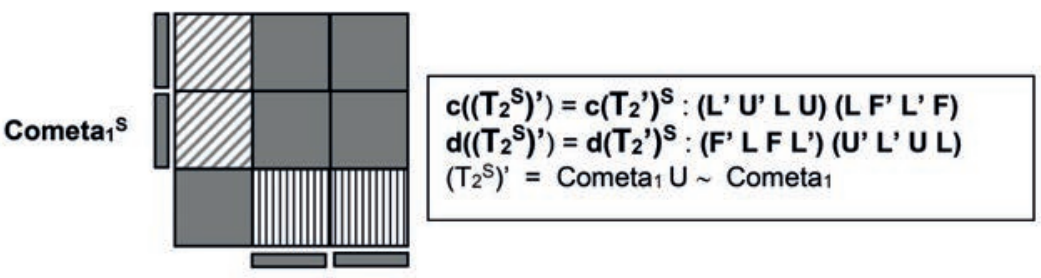

Si se compara esta configuración con la dada en 2.2 se notará que las dos son simétricas (la una es simétrica de la otra):

"El simétrico del inverso es el inverso del simétrico"

2.4.2 Dinámica del constructor de Cometa ${ }_{1}{ }^{\mathrm{S}}, \mathrm{c}\left(\left(\mathrm{T}_{2}{ }^{\mathrm{S}}\right)^{\prime}\right)=$ $\mathrm{d}\left(\mathrm{T}_{2}^{\mathrm{S}}\right)$
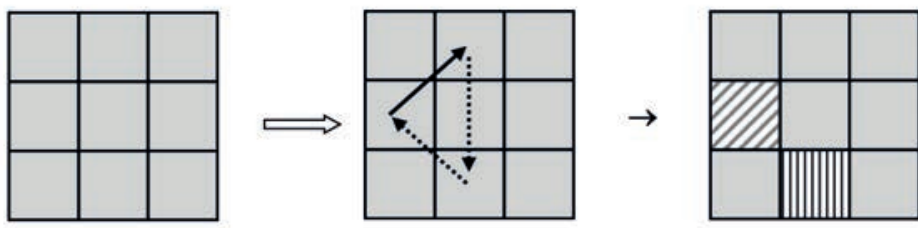

$\mathbf{U}$
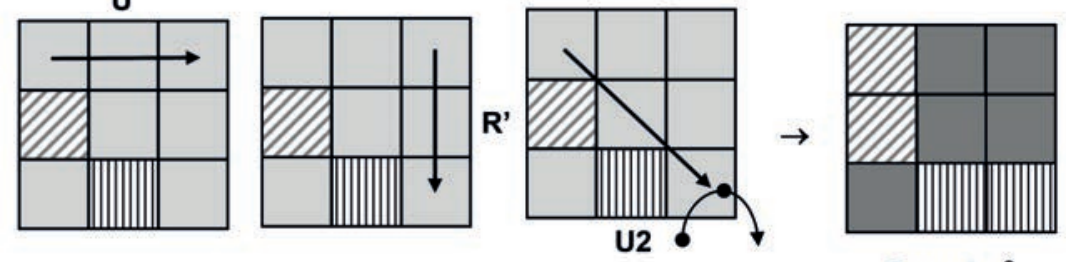

Cometa ${ }^{s}$

$c\left(\left(\mathrm{~T}_{2}{ }^{\mathrm{S}}\right)^{\prime}\right)=\mathrm{c}\left(\mathrm{T}_{2}^{\prime}\right)^{\mathrm{S}}=\mathrm{c}\left(\right.$ Cometa $\left._{1}{ }^{\mathrm{S}}\right)$ y realiza lo de $\mathrm{c}\left(\mathrm{T}_{2}^{\prime}\right)=$ $c\left(\right.$ Cometa $\left._{1}\right)$ pero en sentido simétrico tanto en las aristas como en los vértices. 


\subsubsection{Acción de $\mathrm{c}\left(\right.$ Cometa $\left._{1}^{\mathrm{S}}\right)$ sobre sus aristas iniciales}

$c\left(\right.$ Cometa $\left._{1}{ }^{S}\right)$ realiza una permutación en sentido horario de tres de las aristas distintas de la derecha (permanece invariante), provocando permutaciones en los stickers de algunas.

De modo preciso:

- La arista superior pasa a ser la inferior y sus stickers permutan;

- La arista inferior pasa a ser la izquierda y sus stickers permutan;

- La arista izquierda pasa a ser la superior.

2.4.2.2 Acción de $\mathrm{c}\left(\right.$ Cometa $\left._{1}^{\mathrm{S}}\right)$ sobre sus vértices iniciales $\mathrm{c}\left(\mathrm{Cometa}_{1}{ }^{\mathrm{S}}\right)$ realiza una permutación en sentido horario de tres de los vértices distintos del vértice inferior izquierdo.

De modo preciso:

- El vértice superior izquierdo pasa a ser el vértice superior derecho mediante un movimiento U;

- El vértice superior derecho pasa a ser el vértice inferior derecho mediante un movimiento R';

- El Vértice inferior derecho $\left(\mathrm{V}_{\mathrm{ID}}\right)$ recibe una torsión "horaria" de tres giros (1.6.3.3) y luego gira $180^{\circ}$ en la capa superior mediante un movimiento U2. Lo primero significa:

Los tres stickers del Vértice inferior derecho $\left(\mathrm{V}_{\mathrm{ID}}\right)$ inicial permutan en "sentido horario"

- Su sticker superior inicial pasa a la cara derecha;

- Su sticker derecho inicial pasa la cara frontal; y

- Su sticker frontal inicial pasa a la cara superior. 
A continuación, se analizará los primeros siete "PLL: 2 $\mathrm{U}, 2 \mathrm{~A}, \mathrm{H}, \mathrm{Z}$ y E" teniendo en cuenta que los algoritmos PLL (Permutation of Last Layer:) tienen como tarea organizar las componentes de la última capa del cubo una vez éste se halle totalmente orientado (mediante algoritmos OLL); por ello un PLL lo que hace es acomodar las piezas en sus posiciones originales mediante permutación en la capa superior. En la mayoría de los PLL no se necesita hablar de constructor y de constructor, pues estos resultan iguales.

2.5 Las U: Permutaciones de 3 aristas con los vértices bien acomodados $^{6}$

2.5.1 Ua: Permutación de tres aristas en sentido horario.
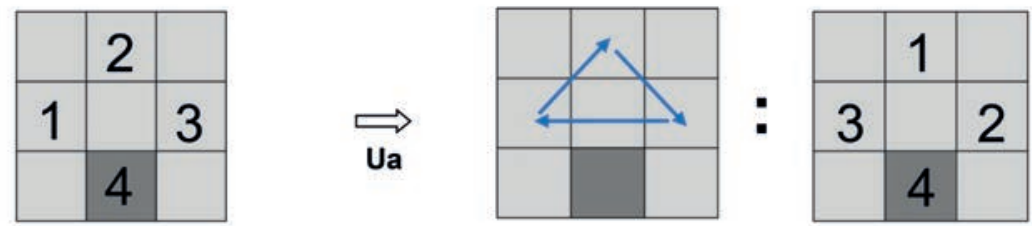

2.5.1.2 Forma Canónica

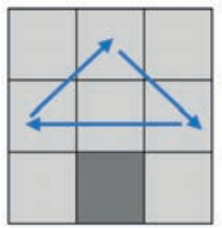

c(Ua): M2 U' M U2 M' U' M2

M

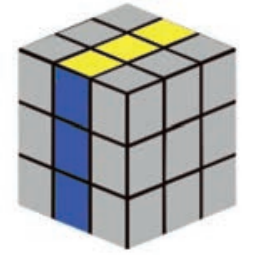

M: Movimiento en sentido horario de la capa del medio paralela a la cara derecha. Es un movimiento parecido al $\mathrm{R}$, pero se realiza con la capa del medio

M': Movimiento inverso al M. Esto es, un Movimiento en sentido antihorario de la capa del medio paralela a la cara derecha. Es un movimiento parecido al R' pero se realiza con la capa del medio.

6 La forma en " $U$ " en estas configuraciones de los lados superiores frontal y laterales, las distinguen de un modo relativamente rápido. 
Con el cubo en su estado original y Ua surge esta configuración:

- La arista inferior queda invariante;

- La arista derecha pasa a ser la arista izquierda;

- La arista izquierda pasa a ser la arista superior;

- La arista izquierda pasa a ser la arista superior.

En otras palabras, con Ua se permutan en sentido horario las tres aristas distintas de la inferior.

Pregunta: ¿Cómo volver al estado original del cubo antes de ejecutar este algoritmo? Permutando las aristas en sentido antihorario, función que realiza el siguiente PLL?

\subsubsection{Ub = Ua': Permutación de tres aristas en sentido} antihorario.

\subsubsection{Motivación}
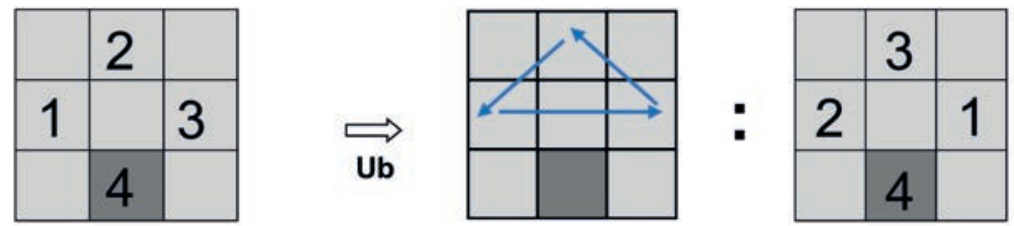

\subsubsection{Forma Canónica}

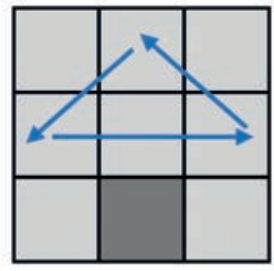

C(Ub): M2 U M U2 M' U M2

$$
\mathrm{Ub}=\mathrm{Ua}^{-1}=\mathrm{Ua}^{\prime}
$$

7 Las figuras indican cómo debe ubicarse el cubo para ejecutar ese algoritmo y cumplir su objetivo. En este caso ubicar de frente la cara lateral que está completamente organizada. El NO ubicar correctamente el cubo para la ejecución de Ub produce efecto de otro PLL 
Con el cubo en su estado original y Ub surge esta configuración:

- La arista inferior queda invariante;

- La arista izquierda pasa a ser la arista derecha;

- La arista derecha pasa a ser la arista superior;

- La arista superior pasa a ser la arista izquierda.

En otras palabras, con Ub se permutan en sentido antihorario las tres aristas distintas de la inferior.

Pregunta: ¿Cómo volver al estado del cubo antes de ejecutar este algoritmo $\mathbf{U b}$ ?

Permutando las aristas en sentido horario, es decir, ejecutando $\mathrm{Ua}(=\mathbf{U b})$.

\subsubsection{Configuración para aplicar los algoritmos " $U$ "}

- Nos concentramos únicamente en la capa superior, especialmente en los lados de arriba de las caras laterales.

- Hay una y sólo una cara lateral donde en su lado de arriba se observa que "todos sus sticker (3) tienen el mismo color" (opuesto al del sticker lateral de una de las aristas laterales). Ese lado de arriba se ubica en la cara frontal del cubo (no importa cómo quede el resto del cubo).

- Al colocar frontalmente el lado antes descrito centramos nuestra mirada en la " $U$ " formada por este lado y los lados superiores de sus caras contiguas izquierda y derecha. Estos dos últimos lados son "ajedrezadas": Los stickers de sus extremos son del mismo color, pero distinto al del sticker central.

- De tener una configuración en la capa superior como la descrita en los dos párrafos anteriores: 
Si en el lado superior de la cara derecho los dos colores distintos (los dos primeros) son opuestos se aplica "Ua"; pero si no son opuestos (los dos primeros) se aplica "Ub".

Al final con movimientos $U$ se organiza totalmente el cubo.

2.6 Las A: Permutaciones de 3 vértices con las aristas bien acomodadas $^{8}$

2.6.1 Aa: Permutación de tres vértices en sentido horario.

\subsubsection{Motivación.}
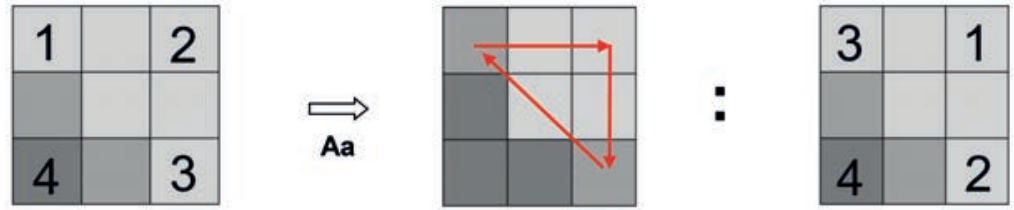

\subsubsection{Forma Canónica}

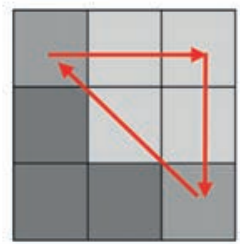

\section{c(Aa) : R' F R' B2 RF' R R2 B2 R2}

Con el cubo en su estado original y Aa surge esta configuración:

- El vértice inferior izquierdo queda invariante;

- El vértice inferior derecho pasa a ser el superior izquierdo;

- El vértice superior izquierdo pasa a ser el superior derecho;

- El vértice superior izquierdo pasa a ser el inferior derecho.

8 La forma triangular frontal en sus configuraciones coincide con la parte triangular superior de una A mayúscula. 
En otras palabras, con Aa se permutan en sentido horario los tres vértices distintos del inferior izquierdo.

- Algo más, Ee vértice inferior izquierdo (el que no cambia) y las aristas izquierda e inferior configuran una $\mathrm{V}$ tridimensional a izquierda ${ }^{9}$.

Pregunta: ¿Cómo volver al estado del cubo antes de ejecutar este algoritmo? Permutando los vértices en sentido antihorario, función que realiza el siguiente PLL.

\subsubsection{Ab = Aa': Permutación de tres vértices en sentido} antihorario.

\subsubsection{Motivación}
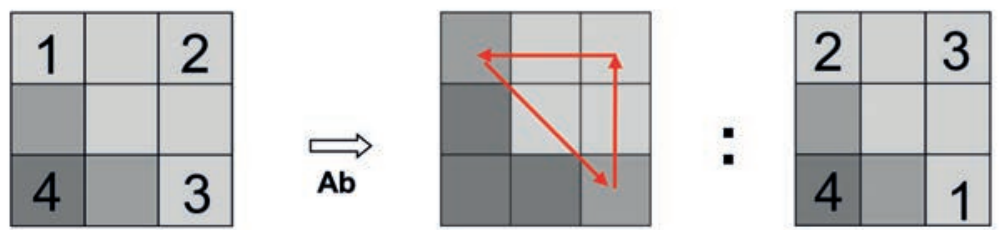

\subsubsection{Forma Canónica}

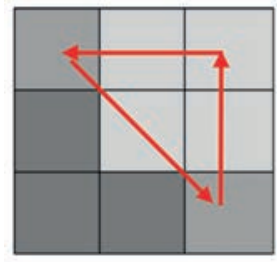

\section{c(Ab): R2 B2 R2 R' F R' B2 R F' R}

Con el cubo en su estado original y Ab surge esta configuración:

- El vértice inferior izquierdo queda invariante;

- El vértice superior izquierdo pasa a ser el inferior derecho;

9 Esto es clave tenerlo en cuenta para saber la posición inicial del cubo cuando se vaya a ejecutar el algoritmo Aa. 
- El vértice superior derecho pasa a ser el superior izquierdo;

- El vértice inferior derecho pasa a ser el superior izquierdo.

En otras palabras, con $\mathrm{Ab}$ se permutan en sentido antihorario los tres vértices distintos del inferior izquierdo.

- Algo más, El vértice inferior izquierdo (el que no cambia) y las aristas izquierda e inferior configuran una $\mathrm{V}$ tridimensional a izquierda.

\subsubsection{Configuración para aplicar los algoritmos " $\mathrm{A}$ "}

- Nos concentramos únicamente en la capa superior, especialmente en los lados de arriba de las caras laterales.

- Hay dos caras laterales contiguas donde en cada uno de sus lados superiores hay un bloque de dos stickers laterales juntos del mismo color ${ }^{10}$. Estos dos bloques configuran una " $V$ " y desde luego tienen un vértice en común; el cual ubicamos en el vértice inferior izquierdo $\left(\mathbf{V}_{\text {I.I. }}\right)$ de la capa superior.

- Ubicado ese vértice común como $\mathbf{V}_{\text {I.I }}$ se observa que los stickers laterales de los otros dos vértices de los lados acá mencionados, "V", tienen el mismo color.

De tener una configuración en la capa superior como la descrita en los dos párrafos anteriores:

\section{Si en lado superior de la cara derecha vemos tres colores distintos (los dos primeros de colores opuestos) se aplica "Aa"; pero si sólo vemos dos colores distintos (los dos primeros de colores NO opuestos) se aplica "Ab". \\ Al final con movimientos $U$ se organiza totalmente el cubo.}

Finalizamos este capítulo dando a conocer tres PLL sumamente útiles como son la "H", la "Z" y la "E".

10 De modo preciso, ese color es el opuesto al del sticker lateral de la arista opuesta a la que se halla en c/u de esos bloques. Ello obedece al hecho de que las aristas están bien acomodadas. 
2.7 La permutación "H": Intercambiando aristas opuestas

2.71 Motivación. $\mathrm{H}=\mathrm{Ub} \mathrm{U}$ Ub U'
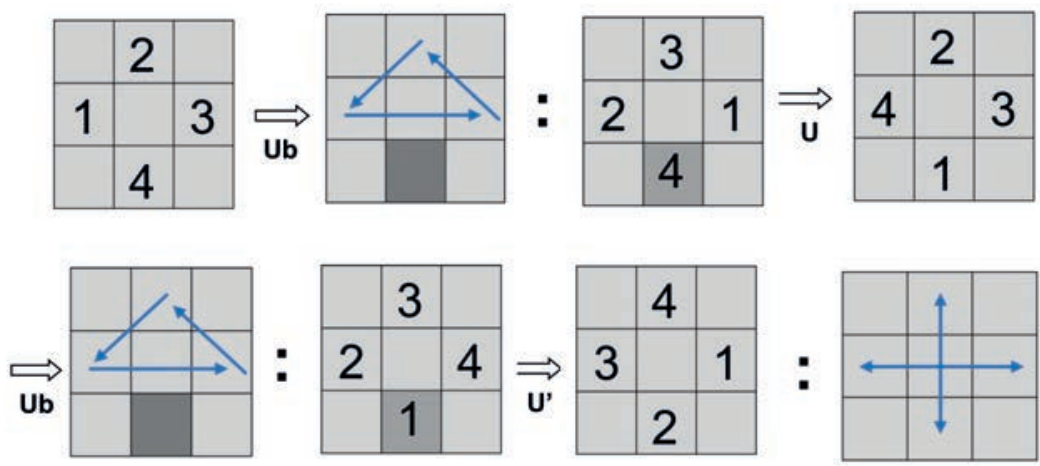

H

Actividad: Ejecutar el algoritmo $\mathrm{H}$ usando el cubo en su estado original. Para retornar al cubo en su estado original vuelva a ejecutar ese algoritmo.

\subsubsection{Canónico de $\mathrm{H}$}

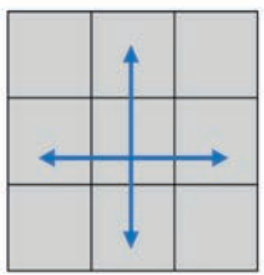

\section{c(H): (M2 U M2) U2 (M2 U M2)}

Su misión es Permutar simultáneamente los dos pares de aristas opuestas.

\subsubsection{Configuración "Ajedrezada con opuestos", aplicar el} algoritmo " $\mathrm{H}$ "

- Nos concentramos únicamente en la capa superior, especialmente en los lados de arriba de las caras laterales (las otras dos capas están ya organizadas y no nos interesan mucho en este momento). 
- En el lado superior de la cara frontal se observa que el sticker lateral no superior de la arista allí presente tiene un color opuesto al de los stickers laterales de los vértices contiguos a dicha arista.

En el lado superior de la cara derecha se observa algo similar a lo que sucede con el de la cara frontal: El sticker lateral no superior de la arista allí presente tiene un color opuesto al de los stickers laterales de los vértices contiguos a dicha arista ${ }^{11}$.

- Esta configuración bien puede denominarse "Configuración ajedrezada con opuestos". De tener una configuración de ese tipo en la capa superior se aplica el algoritmo " $\mathrm{H}$ ". Al final con movimientos U se organiza totalmente el cubo.

\subsection{La permutación "Z": Intercambiando aristas contiguas}

\subsubsection{Motivación: $Z=$ Ub U’ Ub U}
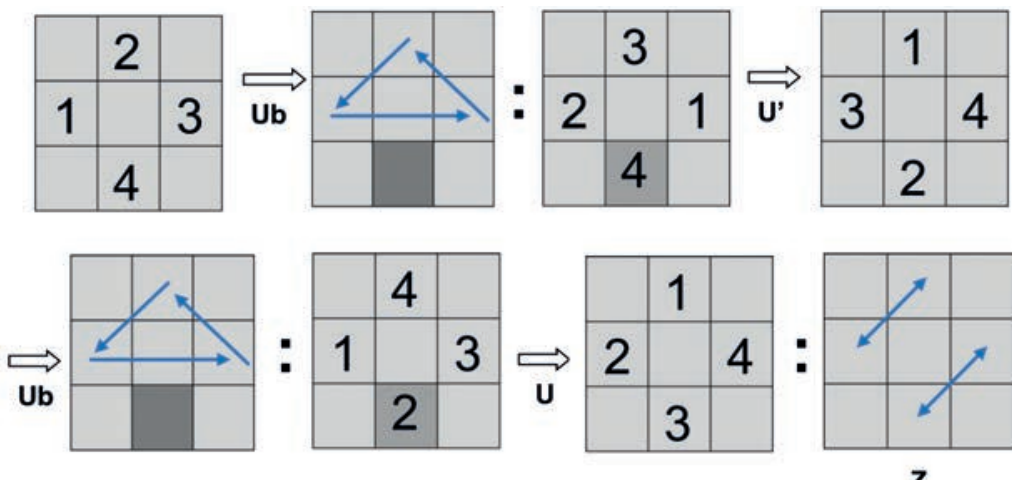

Actividad: Ejecutar el algoritmo $\mathrm{Z}$ usando el cubo en su estado original. Para retornar al cubo en su estado original vuelva a ejecutar el mismo algoritmo.

11 Igual sucede con los otros dos lados. 


\subsubsection{Canónico de Z}

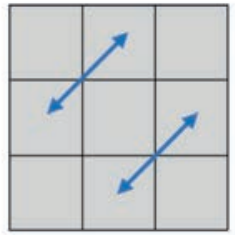

\section{c(Z): (M2 U M2 U) (M U2 M2 U2 MU2)}

Su misión es Permutar simultáneamente los dos pares de aristas contiguas de modo paralelo a la diagonal principal $^{12}$.

Comentario. H: Z U Z U', comprobación para el lector.

\subsubsection{Configuración “Ajedrezada de NO opuestos", aplicar el} algoritmo " $\mathrm{Z}$ "

Es una configuración muy parecida a la de " $\mathrm{H}$ " en el sentido de que los stickers laterales (no superiores) de los vértices de sus lados superiores tienen el mismo color; Su diferencia radica en que los stickers laterales (no superiores) son de color distinto, pero NO opuesto al de los stickers laterales (no superiores) de la arista que está entre ellos.

- Nos concentramos únicamente en la capa superior, especialmente en los lados de arriba de las caras laterales (las otras dos capas están ya organizadas y no nos interesan mucho en este momento).

- Hay dos pares de lados contiguos que forman cada par una "V ajedrezada". De modo preciso, cada par de esos lados tienen stickers laterales de dos colores $\mathrm{NO}$ opuestos y coinciden en un vértice cuyos stickers laterales (no superiores) tienen los dos colores únicos que se ven en la $\mathrm{V}$ ajedrezada. Llamemos a esos vértices como "vértices de interés para $\mathrm{H}$ ".

- Ubicamos uno de los "vértices de interés para H" como vértice inferior derecho de la capa superior.

12 Diagonal principal: Ésta es la que se determina por el vértice inferior izquierdo y por el vértice superior derecho, va de abajo hacia arriba. 
De tener una configuración de ese tipo en la capa superior se aplica el algoritmo " $\mathrm{Z}$ ". Al final con movimientos U se organiza totalmente el cubo.

Si todos los vértices de la capa superior están bien ubicados y se encuentran 2 aristas contiguas de colores laterales intercambiados se aplica el algoritmo " $Z$ ", en el caso contrario se aplicará el algoritmo "H". En cualquiera de los dos casos, se organiza totalmente el cubo.

2.9 La "E": Permutando Vértices Contiguos

2.9.1 Motivación. $E=\mathrm{U}$ Aa U Aa U2

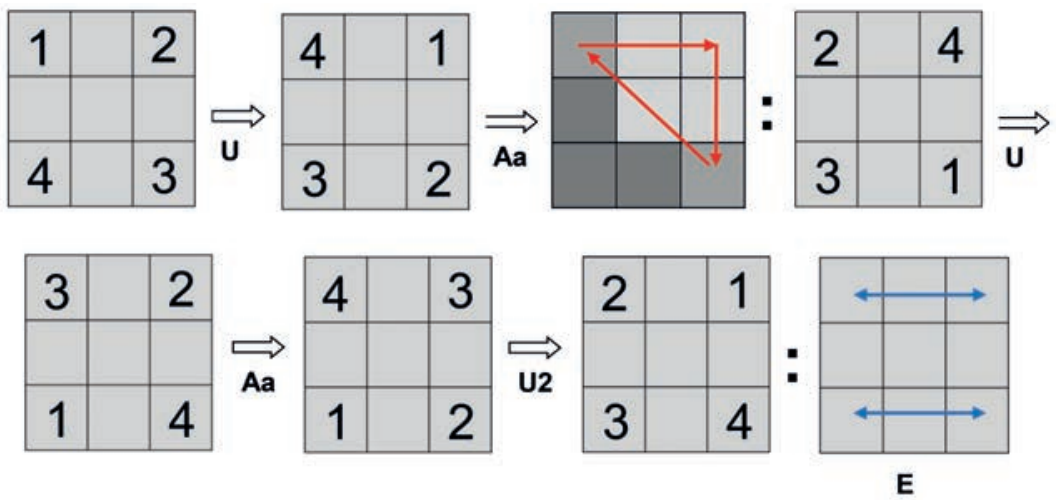

Actividad: Ejecutar el algoritmo E usando el cubo en su estado original. Para retornar al cubo en su estado original se debe volver a ejecutar el mismo algoritmo.

2.9.2 Canónico de $\mathrm{E}$

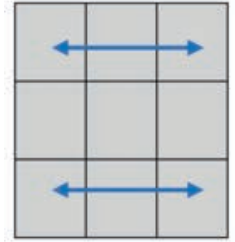

\section{c(E): (R'UL' D2 LU'R) (L'UR' D2 RU'L)}

Su misión es Permutar simultáneamente los vértices inferiores y los vértices superiores. 


\subsubsection{Configuración "Visualmente desordenada", aplicar el algoritmo " $E$ "}

En esta configuración todos los 3 stickers de cualesquiera de los lados superiores de las caras frontales tienen colores distintos, pero dos de estos (los extremos) son opuestos. Precisamente esa primera "visión desordenada" de la configuración "E" facilita su rápida caracterización.

- Nos concentramos únicamente en la capa superior, especialmente en los lados de arriba de las caras laterales (las otras dos capas están ya organizadas y no nos interesan mucho en este momento).

- Hay dos lados que al ser ubicados c/u de ellos de modo frontal nos dejan ver que el color de los stickers derecho e izquierdo de sus vértices superiores coinciden con el color del sticker frontal de la arista que hay entre estos. Dejamos esa arista como arista inferior de la capa superior.

Una vez examinadas estas características y ratificadas que se dan, se aplica el algoritmo "E". Al final con movimientos U se organiza totalmente el cubo

Síntesis tabular "Canónica" (secciones $2.1 \rightarrow 2.4$ )
4 OLL para practicar y memorizar

\begin{tabular}{|c|c|c|}
\hline 2.1.1 $T_{2}$ & $\begin{aligned} \mathbf{c}\left(\mathbf{T}_{2}\right) & =\left(\mathbf{F} \mathbf{R}^{\prime} \mathbf{F}^{\prime} \mathbf{R}\right)\left(\mathbf{U} \mathbf{R} \mathbf{U}^{\prime} \mathbf{R}^{\prime}\right) \\
\mathbf{d}\left(\mathbf{T}_{2}\right) & =\left(\mathbf{R} \mathbf{U} \mathbf{R}^{\prime} \mathbf{U}^{\prime}\right)\left(\mathbf{R}^{\prime} \mathbf{F} \mathbf{R} \mathbf{F}^{\prime}\right)\end{aligned}$ & 㹂㫪 \\
\hline $\begin{array}{c}2.2 .1 \\
\text { Cometa }\end{array}$ & $\begin{aligned} \mathbf{c}\left(\text { Cometa }_{1}\right) & =\left(\mathbf{R} \mathbf{U} \mathbf{R}^{\prime} \mathbf{U}^{\prime}\right)\left(\mathbf{R}^{\prime} \mathbf{F} \mathbf{R} \mathbf{F}\right) \\
\mathbf{d}\left(\text { Cometa }_{\mathbf{1}}\right) & =\left(\mathbf{F} \mathbf{R}^{\prime} \mathbf{F}^{\prime} \mathbf{R}\right)\left(\mathbf{U} \mathbf{R} \mathbf{U}^{\prime} \mathbf{R}^{\prime}\right)\end{aligned}$ & H \\
\hline 2.3.1 $\mathrm{T}_{2}^{\mathrm{s}}$ & $\begin{array}{l}c\left(\mathbf{T}_{2}{ }^{s}\right)=\left(\mathbf{F}^{\prime} \mathbf{L} \mathbf{F} \mathbf{L}^{\prime}\right)\left(\mathbf{U}^{\prime} \mathbf{L}^{\prime} \mathbf{U} \mathbf{L}\right) \\
\mathbf{d}\left(\mathbf{T}_{2}{ }^{\boldsymbol{S}}\right)=\left(\mathbf{L}^{\prime} \mathbf{U}^{\prime} \mathbf{L} \mathbf{U}\right)\left(\mathbf{L}^{\prime} \mathbf{F}^{\prime} \mathbf{L}^{\prime} \mathbf{F}\right)\end{array}$ & 奉春 \\
\hline$\stackrel{2.4 .1}{\text { Cometa }_{1} \text { s }}$ & 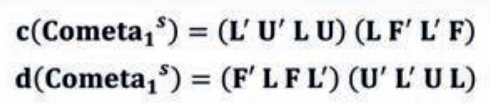 & \\
\hline
\end{tabular}


Síntesis tabular (secciones $2.5 \rightarrow 2.9$ )

7 PLL para practicar y memorizar

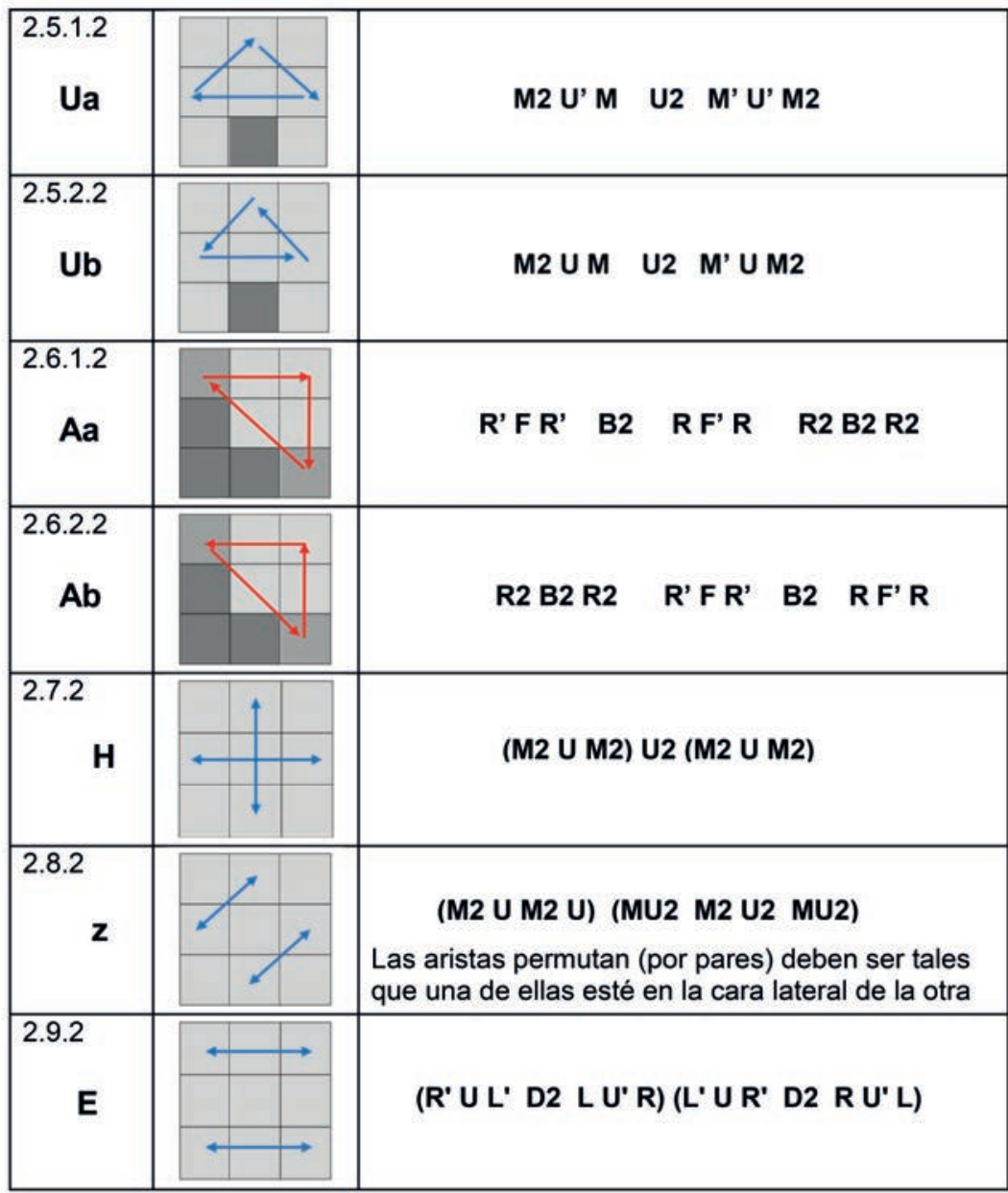




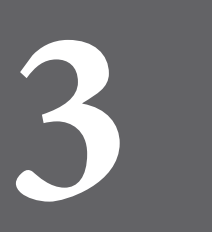

CAPÍTULO

TRES 


\section{Operaciones OLL/PLL y Configuraciones semejantes}

En este capítulo se examinará 3 clases de configuraciones (semejantes, cuasi semejantes y congruentes) y tres operaciones algebraicas las cuales facilitan el estudio y la buena comprensión de todos los OLL/PLL posibles reduciéndolos esencialmente a "los 57 OLL y 21 PLL clásicos", maravilloso trabajo de Jessica Fridrich en 1981.

Podemos afirmar que "La semejanza" es una fuente natural de los OLL y PLL, permitiendo admirar, reconocer y manejar de modo activo ese maravillo trabajo de Fridrich. Para ilustrar las operaciones y conceptos algebraicos acá introducidos se usará algunos de los algoritmos tratados en los dos capítulos anteriores.

Como bien se plantea en la introducción del libro, de modo pragmático y resumido es el querer de este trabajo el poder mostrar la validez de la afirmación siguiente: "La 3 a capa del cubo se orienta y organiza totalmente con 4 algoritmos: $T_{1}, T_{2}, U a y$ Aa”. Para ello se requiere una buena operatividad algebraica y un exquisito manejo de los tópicos que se brinda en el presente capítulo. Las 4 configuraciones antes mencionadas se consideran "primitivas" (no se pueden deducir de las hasta acá conocidas); las configuraciones restantes del libro se obtienen operando tales OLL y PLL; en ese sentido se aplica el método deductivo y experimental, propio de las matemáticas, para obtener los resultados requeridos. 


\subsection{Tres Operaciones fundamentales en OLL/PLL}

\subsubsection{La operación Conjunción ${ }^{13}$ entre algoritmos OLL / PLL}

Sean p y q algoritmos OLL. Definimos la siguiente operación conjunción $(*)$ entre ellos

$$
\mathbf{p} * \mathbf{q}=\mathbf{r}=\mathbf{p q}
$$

donde "pq" se logra uniendo los componentes de p y q. Se activa realizando primero el algoritmo p, y luego sobre la configuración resultante se ejecuta el algoritmo q.

El algoritmo "pq" resulta ser otro OLL si uno de ellos lo es OLL y el otro es un PLL, y es un PLL si ambos lo son (en cuyo caso los PLL conforman un "grupo" matemático de permutaciones).

Este resultado es comprensible si entendemos que los OLL/ PLL trabajan en la capa superior, sin afectar la organización de las capas inferior e intermedia, produciendo nuevas configuraciones o deconstruyendolas o cambiando de lugar a las componentes de esa cara superior.

Un caso particular de esta operación es la Potencia de un algoritmo: $\mathrm{pN}$.

- La operación Potencia de un algoritmo: pN: Consiste en operar el algoritmo p consigo mismo $\mathrm{N}$ veces.

\section{$p N=p p \ldots p$ \\ $\mathbf{N}$ veces}

13 El nombre de esta operación procede de la lingüística. Conjunción: Palabra que se utiliza para unir dos o más partes de una oración. Toda oración es conjunción de palabras, y toda palabra es conjunción de letras. 


\subsubsection{La operación externa $*$ entre configuraciones y}

\section{algoritmos OLL}

Sean $\mathrm{C}$ una configuración OLL, $\mathrm{C}=\mathrm{C}_{\mathrm{p}}$, con $\mathrm{p}$ un OLL/PLL, y q otro algoritmo OLL/PLL.

Se define la siguiente operación entre ellos

$$
\mathrm{C} * \mathrm{q}=\mathrm{C}_{\mathrm{p}} * \mathrm{q}=\mathrm{C}_{\mathrm{r}} \text { donde } \mathbf{r}=\mathbf{p q}
$$

\section{La operación adición (interna) entre configuraciones OLL/PLL}

Sean $C=C_{p}$ una configuración OLL/PLL ( $p$ un OLL/PLL), y $\mathrm{D}=\mathrm{C}_{\mathrm{q}}$ otra configuración OLL/PLL ( $\mathrm{q}$ un OLL/PLL). Se define la siguiente operación entre ellas:

$$
\mathrm{C}+\mathrm{D}=\mathrm{C}_{\mathrm{p}}+\mathrm{C}_{\mathrm{q}}=\mathrm{C}_{\mathrm{r}} \text { donde } \mathrm{r}=\mathbf{p q}
$$

\subsection{OLL Semejantes y clases de equivalencia por semejanza}

Inicialmente se puede afirmar que dos algoritmos OLL $\mathrm{p}$ y $\mathrm{q}$ son semejantes $(\mathrm{p} \sim \mathrm{q})$ si y sólo si tanto $\mathrm{q} * \mathrm{p}^{\prime}$ como $\mathrm{p} * \mathrm{q}^{\prime}$ son PLL.

Notando que si $\mathrm{q} * \mathrm{p}$ ' es un PLL de inmediato $\mathrm{p} * \mathrm{q}$ ' también lo es (aceptando que el inverso de un PLL es otro PLL ${ }^{14}$ ):

$$
\mathrm{qp}^{\prime}=\mathrm{r}_{1} \mathrm{PLL} \Rightarrow \mathrm{q} \mathrm{r}_{1}^{\prime}=\mathrm{p} \Rightarrow \mathrm{r}_{2}=\mathrm{r}_{1}{ }^{\prime}=\mathrm{pq}^{\prime} \Rightarrow \mathrm{pq}^{\prime}=\mathrm{r}_{2}=\mathrm{r}_{1}{ }^{\prime} \mathrm{PLL}
$$

Simplemente se afirma:

\subsubsection{Definición 1}

Dos algoritmos OLL p y q se consideran semejantes ( $\mathrm{p}$ q) si y sólo si $q * p^{\prime}=r$ para algún PLL $r$.

Dicho de otro modo, $\mathrm{p} \sim \mathrm{q}$ si y solo si se cumple al menos una de estas dos condiciones ${ }^{15}$ :

14 No es difícil aceptar que el inverso de un PLL es otro PLL, pues la misión de un PLL es permutar las componentes de la última capa sin afectar sus orientaciones ni las otras dos capas; y el inverso de un PLL lo que hace es el deshacer lo que hace la permutación original dejando al cubo en su estado anterior (cubo totalmente orientado por supuesto). No obstante, cuando se estudien más PLL se podrá constatar lo acá expuesto

15 Si una de esas condiciones se cumple, de inmediato la otra se cumple. 
a) La acción conjunta de q y el inverso de p da el cubo totalmente orientado $\mathrm{C}_{\text {T.O. }}$ (no necesariamente bien organizado: $\mathrm{C}_{\mathrm{I}}$ ) el cual con un PLL $\mathrm{s}_{1}$ vuelve a su estado original

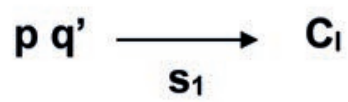

b) La acción conjunta de p y el inverso de q da el cubo totalmente orientado $\mathrm{C}_{\text {T.O. }}$ (no necesariamente bien organizado: $\mathrm{C}_{\mathrm{I}}$ ) el cual con un PLL $\mathrm{s}_{2}$ vuelve a su estado original

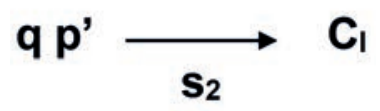

Comentario: Realmente la última condición sobra, pero de ahora en adelante se usará las dos para descifrar la semejanza de dos configuraciones OLL; Esto con el objetivo de adquirir destrezas en el manejo de los constructores/deconstructores canónicos de la configuración.

Se plantea a continuación dos preguntas elementales, pero a la vez fundamentales para comprender este importante concepto de semejanza.

Pregunta 1. ¿Cómo construir un OLL semejante a otro dado $\mathrm{p}$ ?

Rta/ $\mathrm{q}=\mathrm{r} \mathrm{p}$, con $\mathrm{r}$ un PLL, resulta ser semejante a $\mathrm{p}: \mathrm{q} \sim \mathrm{r}$

\section{Pregunta 2}

Supongamos que ya sabemos algebraicamente que $\mathrm{p} \sim \mathrm{q}$ (pq' es un PLL), ¿Se puede reconocer a nivel geométrico o a primera vista que tales algoritmos son semejantes?

Rta/ Cuando $\mathrm{p} \sim \mathrm{q}$ las configuraciones OLL Cp y Cq en apariencia son las mismas en cuanto a la figura que resulta de la acción de estos PLL con los stickers del color de la cara superior de las componentes que quedaron bien orientadas, y la disposición (posición y torsión) de las piezas que no lo 
quedaron. "Tienen diferencias las configuraciones, pero a veces se pasan por alto y se cree a "primera vista" que son iguales"

Se precisa esto definiendo:

\subsubsection{Configuración por semejanza: $\widetilde{c_{p}}$ :}

Es el registro visual sobre la capa superior generado por un algoritmo OLL/PLL p cuando la observación se limita únicamente a los stickers (de las componentes de la cara superior) cuyo color coinciden con el de la cara superior, y a la disposición (posición y torsión) de las componentes de la capa superior que no quedaron orientadas; olvidando las otras capas y los otros stickers cuyo color no son los de la cara superior.

De ese modo se puede plantear que dos algoritmos OLL son semejantes si y solo si sus configuraciones por semejanza son iguales:

$$
\mathbf{p} \sim \mathbf{q} \Leftrightarrow \widetilde{\boldsymbol{C}_{p}}=\widetilde{\boldsymbol{C}_{q}}
$$

Cada vez que se hable de un OLL estas configuraciones se van a representar gráficamente en dos dimensiones. Y para evitar complicaciones con tales notaciones a esas configuraciones se les asignan su propio nombre ${ }^{16}\left(\mathrm{La}\right.$ “ $\mathrm{T}_{1}$ ”, la “ $\mathrm{T}_{2}$ ”, la “H”, etc.)

A nivel de configuraciones: PLL + OLL $\mathbf{A} \sim \mathbf{O L L} \mathbf{A}$

3.3 La semejanza: Una relación de equivalencia. Invarianza de la simetría por semejanzas

\subsubsection{Propiedades de La semejanza}

Esta relación por lo planteado en (1) de la sección anterior 2.2.2 es una "relación de equivalencia" para los OLL, pero acá se verificará con el empleo del álgebra.

16 Esos nombres no son creados por los autores del presente libro, sino que ya son conocidos desde hace muchos años. 
a. La semejanza es reflexiva: $p \sim p$, cuando $p$ es un algoritmo OLL.

Esto es válido por definición. Nótese además que:

$$
C_{p^{\prime}}=C_{1} \text { : Cubo totalmente organizado }
$$

b. La semejanza es simétrica: Si $p \sim q \Rightarrow q \sim p$, cuando p y q son algoritmos OLL. En efecto:

$$
\begin{aligned}
\text { Si } p \sim q \Rightarrow & q=r p \text { para algún PLL } r \\
& \Rightarrow r^{\prime} q=p \\
& \Rightarrow p=s q \text { donde } s=r^{\prime} \text { otro PLL } \\
\Rightarrow q & \sim p
\end{aligned}
$$

c. La semejanza es transitiva: Si $p_{1} \sim p_{2}$ y $p_{2} \sim p_{3} \Rightarrow$ $p_{1} \sim p_{3}$, cuando $p_{1} p_{2}$ y $p_{3}$ son algoritmos OLL. En efecto:

Si $p_{1} \sim p_{2}$ y $p_{2} \sim p_{3} \Rightarrow$ existen PLL $r_{1}$ y $r_{2}$ tales que

$$
\begin{array}{ll} 
& p_{2}=r_{1} p_{1} \quad \text { y } p_{3}=r_{2} p_{2} \\
\Rightarrow \quad & p_{3}=r_{2} r_{1} p_{1=} r p_{1^{\prime}} \\
& \text { donde } r=r_{2} r_{1} \text { otro } P_{L L}{ }^{17}
\end{array}
$$

\subsubsection{La conservación de la semejanza por simetría}

Esto obedece a la definición de semejanza y a la propiedad "simétrica" de los PLL:

\section{sí $r$ es un PLL, su simétrico $\mathrm{r}^{\mathrm{S}}$ también lo es (1)}

La configuración del simétrico de un PLL es el simétrico de la configuración del original; $\mathrm{r}^{\mathrm{S}}$ opera simétricamente con las componentes de la última capa al modo como lo hace el PLL original $r$.

17 Como la misión de un PLL es permutar las componentes de la última capa sin afectar su orientación ni las otras dos capas, al actuar dos PLL se va a obtener una reordenación de las componentes de la capa superior sin afectar las otra dos. 
La afirmación (1) se puede probar de modo experimental con cada uno de los PLL que se vayan desarrollando.

Habiendo aceptado (1) y recordando que $(\mathrm{pq})^{\mathrm{S}}=\mathrm{p}^{\mathrm{S}} \mathrm{q}^{\mathrm{S}}$ y $\left(\mathrm{p}^{\prime}\right)^{\mathrm{S}}=\left(\mathrm{p}^{\mathrm{S}}\right)^{\prime}$ para algoritmos $\mathrm{p}$ y q se verificara la siguiente propiedad:

Si $p \sim q, p^{s} \sim q^{s}$.

En efecto:

$$
\begin{aligned}
\mathrm{p} \sim \mathrm{q} & \Rightarrow q p^{\prime}=r P L L \\
& \Rightarrow\left(q p^{\prime}\right)^{s}=r^{s} P L L \\
& \Rightarrow q^{s}\left(p^{\prime}\right)^{s}=r^{s} \\
& \Rightarrow q^{s}\left(p^{s}\right)^{\prime}=t \text { PLL, donde } t=r^{s} \\
\Rightarrow p^{s} & \sim q^{s} \text { (por definición). }
\end{aligned}
$$

\subsection{Clases de equivalencias y algoritmos OLL canónicos}

\subsubsection{Clase de p: $[\mathrm{p}]$}

La relación de semejanza permite organizar los OLL en conjuntos de OLL que sean semejantes entre sí y de un modo preciso semejantes a uno dado. Esos conjuntos se denominan "Clases de equivalencias por semejanza" y se notan [p]

$$
[\mathbf{p}]=\{\mathbf{q} \text { OLL }: \mathbf{q} \sim \mathbf{p}\}
$$

\subsubsection{Configuración de $[\mathrm{p}]$}

Como todos los elementos de esa clase tienen la misma configuración por semejanza (sección 2.2.2.), la configuración de "p" por semejanza bien puede denominarse configuración de la clase generada por $p$

$$
\mathbf{C}_{[\mathrm{p}]}=\widetilde{\boldsymbol{C}_{p}}
$$




\subsubsection{Pregunta}

¿Cuántas configuraciones de [p] se conocen?

Rta/: Esencialmente 57 ("Los 57 clásicos algoritmos OLL"). La relación de semejanza clasifica los OLL en conjuntos que no se interceptan entre sí. Eso permite considerar de cada clase un elemento que represente a todos los demás de dicha clase, para poderla distinguir de las otras clases y realizar operaciones entre clases. Ese elemento notado $\hat{\boldsymbol{p}}$ (para [p]) se denomina "Canónico" y trata de caracterizarse por ser el algoritmo más "simple", "sencillo" y "manejable".

- Configuración canónica $\widehat{\boldsymbol{c}_{p}}$ :Es la configuración generada por el "canónico" de la [p].

El hallar canónicos de un algoritmo fue el extraordinario trabajo de Jessica Fridrich, al poder distinguir esencialmente 57 OLL "canónicos", que junto con 21 PLL permiten trabajar con cualquier otro OLL y PLL; todos ellos se presentan en este libro como una segunda forma para construir y deconstruir la configuración OLL, que en el momento se esté analizando (la primera forma se va a generar a partir del método deductivo y los algoritmos básicos $\mathrm{T}_{1}, \mathrm{~T}_{2}, \mathrm{U}_{\mathrm{a}} \mathrm{y} \mathrm{U}_{\mathrm{b}}, \mathrm{A}_{\mathrm{a}} \mathrm{y}_{\mathrm{b}}$ mediante la operación conjunción).

\subsubsection{Propiedad fundamental del "canónico" de una clase}

Si $\mathrm{p}_{1} \in[\hat{p}]$, el deconstructor $(\hat{p}$ ') de la configuración generada por $\hat{p}, \widehat{C \hat{p}}$, también deconstruye a la configuración generada por $\mathrm{p}_{1}, \widetilde{C p_{1}}$

Locual es evidente por la igualdad entre tales configuraciones. 
De esa manera tenemos:

si $\mathbf{t}=\mathbf{p}_{\mathbf{1}} \hat{\boldsymbol{p}}, \quad \Rightarrow \mathbf{C}_{\mathbf{t}}=\mathbf{C}_{\mathbf{T}} \mathbf{} \mathbf{}, \quad(\text { cubo totalmente orientado })^{18}$

Lo anterior justifica el por qué un cubo de rubik se vuelve tan interesante y agradable para ser manejado visualmente, pues muchas de las configuraciones son realmente distintas pero semejantes (idénticas a primera vista), por la razón anterior se vuelven "idénticas" para su deconstrucción.

Una forma de descubrir nuevos PLL: Conjuntando el constructor OLL $\mathbf{p}$ de una configuración $\mathrm{C}_{1}$ con el deconstructor q' de una configuración $\mathrm{C}_{2}$ semejante a $\mathrm{C}_{1}$, generada por un constructor $\mathrm{q} \sim \mathrm{p}$ :

$$
\text { p q' }
$$

Este método es el que se empleará para hallar algún PLL, como bien se especificará desde el capítulo 4 , solo que una de esas formas a emplear es la canónica:

$$
c(C p) * \widehat{d}(C p)
$$

\subsection{Configuración cuasi semejante y su nomenclatura indexada}

Son configuraciones de idénticas figuras diseñadas con las componentes bien orientadas de la capa superior, pero que no son semejantes (al examinar las componentes no orientadas se presentan diferencias). Ejemplo, $\mathrm{T}_{1} \mathrm{y} \mathrm{T}_{2}$ son cuasi semejantes.

"Para poder distinguir esas cuasi semejantes" después de cada nombre se colocan cuatro valores números naturales $\left(n_{1}, n_{2}, n_{3}\right.$, $\mathrm{n}_{4}$ ) donde:

$\mathrm{n}_{1}$ : número de componentes con torsión frontal;

$\mathrm{n}_{2}$ : número de componentes con torsión derecha;

$\mathrm{n}_{3}$ : número de componentes con torsión trasera;

$\mathrm{n}_{4}$ : número de componentes con torsión izquierda.

$18 \mathrm{p}_{1} \sim \hat{p}$, luego $\mathrm{t}=\mathrm{p}_{1} \hat{p} \hat{p}^{\prime}$ es un PLL por definición de semejanza. 
En las figuras que se muestran a continuación, se ilustran con sus nuevas nomenclaturas así indexadas, las $\mathrm{T}_{1}$ y T2 tratadas en las secciones 1.4.1 y 1.4.3 del capítulo anterior; y dos I (una de ellas la de la sección 2.7; La otra posteriormente se analizara).

Resulta claro que dos configuraciones semejantes tienen el mismo nombre y la misma indexación ("cuaternas iguales"), y para diferenciarlas en ese caso no sirve tal indexación.

Si dos configuraciones son cuasi semejantes, pero no semejantes sus cuaternas entonces son distintas; ya en esa situación podemos distinguir dos configuraciones cuasi semejantes usando el mismo nombre, pero con distinta indexación.

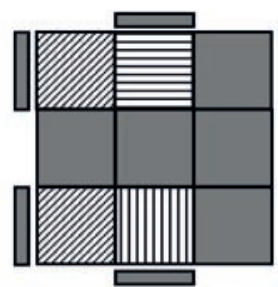

$T_{1}: T(1,0,1,2)$

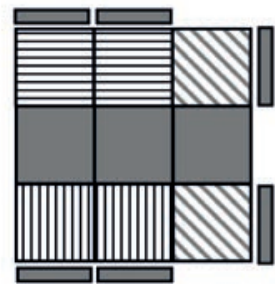

$I_{1}: I(2,2,2,0)$

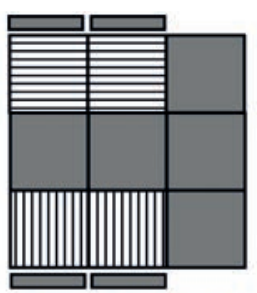

$T_{2}: \quad T(2,0,2,0)$

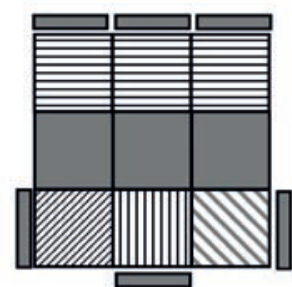

$I_{3}: I(1,1,3,1)$

3.6 Congruencia de dos OLL/PLL. Configuraciones congruentes

b.1.1 Definición. Dos algoritmos OLL p y q son congruentes

$$
\mathbf{p} \cong \mathbf{q}
$$

sí y sólo si uno de ellos se obtiene del otro por rotación en la cara superior. Dicho de otra manera: 
$\mathbf{p} \cong \mathbf{q}$ si y sólo si existe un entero positivo m comprendido entre 1 y 4 tal que

$$
\mathbf{q}=(\mathrm{U}) \mathrm{m} \mathrm{p}
$$

En cuyo caso "la configuración de uno de ellos es la misma del otro" (excepto una rotación de la capa superior).

b.1.2 La congruencia: Una relación de equivalencia. Invarianza de la congruencia por simetrías e inversos.

b.1.2.1 La congruencia una relación de equivalencia

(prueba para el lector).

Sugerencias:

$$
\begin{aligned}
& {[(\mathrm{U}) \mathrm{m}]^{\prime}=(\mathrm{U})(4-\mathrm{m})} \\
& {\left[(\mathrm{U}) \mathrm{m}_{1}\right]\left[(\mathrm{U}) \mathrm{m}_{2}\right]=(\mathrm{U})\left(\mathrm{m}_{1}+\mathrm{m}_{2}\right)}
\end{aligned}
$$

3.6.2.2 Conservación de la congruencia por inversos.

$$
\begin{gathered}
\mathrm{p} \cong \mathrm{q} \Rightarrow \mathrm{p}^{\prime} \cong \mathrm{q}^{\prime} \\
\text { (Para el lector). Sugerencia: } \\
\mathrm{p} \cong \mathrm{q} \Rightarrow \mathrm{q} \cong \mathrm{p} \text {. Luego, } \mathrm{p}^{\prime} \mathrm{q}^{\prime}=(\mathrm{q} * \mathrm{p})^{\prime}=\left((\mathrm{Um})^{\prime}=(\mathrm{U})(4-\mathrm{m})\right.
\end{gathered}
$$

3.6.2.3 Conservación de la congruencia por simétricos.

$$
\mathrm{p} \cong \mathrm{q} \Rightarrow \mathrm{p}^{\mathrm{S}} \cong \mathrm{q}^{\mathrm{S}}
$$

(Para el lector).

\subsubsection{Configuraciones OLL/PLL congruentes (o Idénticas)}

Sean $\mathrm{C}_{1}$ y $\mathrm{C}_{2}$ las configuraciones resultantes de los algoritmos OLL/PLL p y q. Decimos que son congruentes, $\mathrm{C}_{1} \cong \mathrm{C}_{2}$, si y sólo si sus algoritmos generadores p y q lo son.

$$
\mathbf{C}_{\mathbf{p}} \cong \mathbf{C}_{\mathbf{q}} \quad \Leftrightarrow \mathrm{p} \cong \mathrm{q}
$$


Geométricamente dos configuraciones congruentes son idénticas excepto una rotación de la capa superior.

Desde el punto de vista algebraico puede afirmarse

$$
\begin{aligned}
& \mathbf{C}_{\mathbf{p}} \cong \mathbf{C}_{\mathbf{q}} \quad \Leftrightarrow \quad \mathbf{C}_{\mathbf{p q}},=\mathrm{C}_{\mathrm{I}} \text { (cubo en su estado original) } \\
& \text { ó } \mathrm{Cpq} \text {, }=\mathrm{C}_{\mathrm{I}} \text { excepto una rotación }
\end{aligned}
$$

En aras de relacionar mejor estos conceptos de semejanza y congruencia, seguidamente se presentará dos ilustraciones usando las $\mathrm{T}$ ya conocidas en los dos capítulos anteriores.

\subsection{Nuevos constructor/deconstructor para $T_{1}$}

\subsubsection{Motivación}

Dinámica de $\hat{\mathbf{c}}\left(\mathrm{T}_{1}\right)$ : Se Trabajará con el constructor canónico de $\mathrm{T}_{1}$ (según lo expuesto en 1.6.3) y se aplica a la primera figura de la izquierda de más abajo.
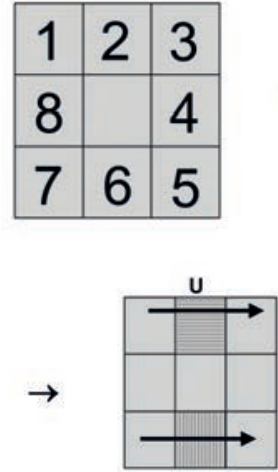

U'
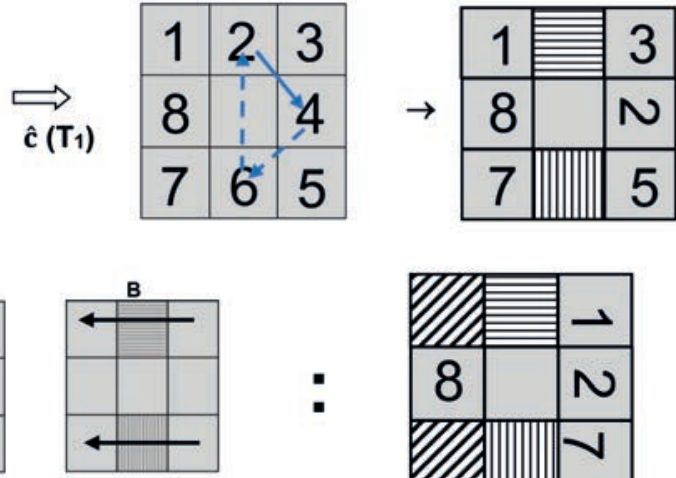

$F^{\prime}$

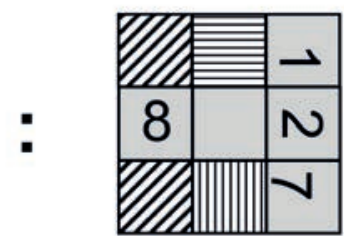

En síntesis:
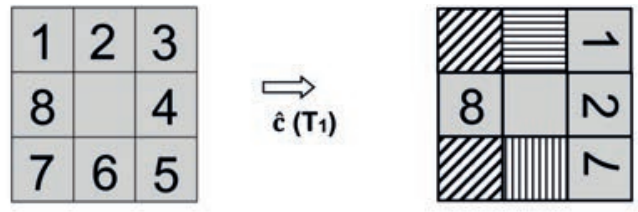

Dinámica de $\mathrm{c}\left(\mathbf{T}_{\mathbf{1}}\right)=\mathrm{Ub} \mathbf{c}\left(\mathbf{T}_{1}\right)$. Jugando de un modo similar, y aprovechando la síntesis de $(1)$, resulta $\mathrm{c}\left(\mathrm{T}_{1}\right)$ un constructor de $\mathrm{T}_{1}$. 


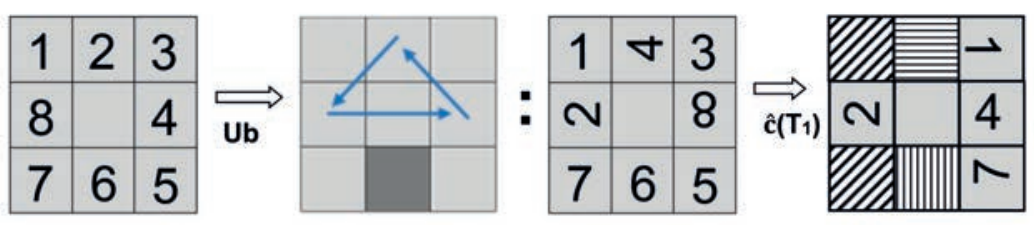

En síntesis:
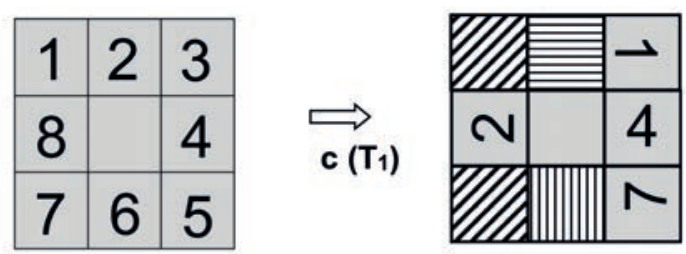

Observación: Los dos algoritmos $\hat{c}\left(\mathrm{~T}_{1}\right)$ y $\mathrm{c}\left(\mathrm{T}_{1}\right)$ realmente construyen a $\mathrm{T}_{1}$, $\mathrm{y}$ sus configuraciones son semejantes pero no congruentes. La diferencia de estas dos radica en sus aristas izquierdas y derechas: Con $\hat{\mathrm{c}}\left(\mathrm{T}_{1}\right)$ la arista izquierda es invariante (la derecha no) en tanto que con $\mathrm{c}\left(\mathrm{T}_{1}\right)$ la arista izquierda NO es invariante (la derecha sí).

\subsubsection{Dos formas de construir/deconstruir $\mathrm{T}_{1}{ }^{19}$}

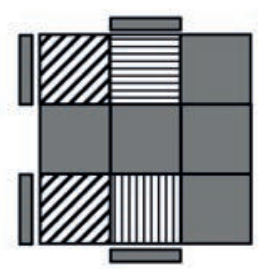

$T_{1}$

\section{$1^{\text {a }}$ Forma}

$$
\begin{aligned}
& c\left(T_{1}\right)=U_{b} \hat{c}\left(T_{1}\right) \\
& d\left(T_{1}\right)=\hat{d}\left(T_{1}\right) U_{a}
\end{aligned}
$$

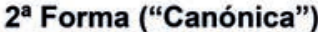

$\hat{\mathbf{c}}\left(\mathrm{T}_{1}\right)=$ F U R U' R' F'

$\hat{d}\left(T_{1}\right)=F R \cup R ' U^{\prime} F^{\prime}$

$(*)$

$$
\begin{aligned}
& \mathbf{U}_{\mathrm{b}} \hat{\boldsymbol{c}}\left(\mathbf{T}_{1}\right)=\left(\text { M2 U M U2 } \mathbf{M}^{\prime} \text { U M2) (F U R U' R' F'), } \mathbf{y}\right. \\
& \hat{\mathbf{d}}\left(\mathbf{T}_{1}\right) \mathbf{U}_{\mathrm{a}}=\text { (F R U R' U' F') (M2 U' M U2 M' U' M2) }
\end{aligned}
$$

\subsubsection{La no congruencia de las dos formas}

En la configuración de la $2^{\text {a }}$ forma la arista izquierda (de la cara superior) está bien acomodada (bien posicionada y sin torsión), lo que no sucede con la $1^{\text {a }}$ forma.

19 Los dos algoritmos de la $2^{\text {a }}$ forma que se presenta en este libro son más simplificados que la primera y se debe en gran parte al trabajo de Jessica Fridrich. 
3.7.4 La semejanza en la configuración de las dos formas Intercambiando constructor/deconstructor.

Sean $\mathbf{m}=\mathbf{c}\left(\mathbf{T}_{1}\right)=\mathrm{Ub} \hat{\mathbf{c}}\left(\mathbf{T}_{1}\right)$ y $\mathbf{n}=\hat{\mathbf{c}}\left(\mathbf{T}_{1}\right)=\mathrm{F}$ U R U' R' F'.

Por definición 3.2.1 m $\sim \mathrm{n}\left(\mathrm{m} * \mathrm{n}^{\prime}=\mathrm{Ub} \mathrm{PLL}\right)$. Algo más, al ejecutar los constructores de ambas formas se obtiene la misma configuración por semejanza:

$$
\widetilde{C_{m}}=\widetilde{C_{n}}
$$

A continuación, se verificará lo anterior también de modo algebraico Intercambiando constructor/deconstructor.

\subsubsection{Situación 1. p: $\mathrm{c}\left(\mathrm{T}_{1}\right) \hat{d}\left(\mathrm{~T}_{1}\right)$, donde}

$$
\begin{gathered}
\mathrm{c}\left(\mathrm{T}_{1}\right)=\mathrm{Ub} \hat{\mathrm{c}}\left(\mathrm{T}_{1}\right)=\mathrm{Ub} \text { F U R U' R' } \mathrm{F}^{\prime} \\
\hat{d}\left(\mathrm{~T}_{1}\right)=\mathrm{F} \text { R U R' U' F' }
\end{gathered}
$$

Diseñar la configuración $I_{1}$ a partir de la $1^{\text {a }}$ forma (constructor $\mathrm{c}\left(\mathrm{I}_{1}\right)$ ) y orientar el cubo usando la $2^{\mathrm{a}}$ forma (deconstructor $\hat{d}\left(\mathrm{I}_{1}\right)$ ).

Sea $\mathrm{C}_{\mathrm{p}}$ la configuración resultante de ejecutar $\mathbf{p}(=\mathrm{Ub})$ desde el cubo en su estado original. Sucede que $\mathrm{C}_{\mathrm{p}}=\mathrm{C}_{\text {T.o. }}$ (cubo totalmente orientado, pero no totalmente organizado).

$$
c\left(\mathrm{~T}_{1}\right) \hat{\mathrm{d}}\left(\mathrm{T}_{1}\right) \longrightarrow \mathrm{C}_{\mathrm{T} . \mathrm{O}}
$$

Para llevar el cubo a su estado original puede aplicarse el PLL: Ua

$$
c\left(T_{1}\right) \hat{d}\left(T_{1}\right) \longrightarrow C_{I}
$$

3.7.4.2 Situación 2. q: $\hat{c}\left(\mathrm{~T}_{1}\right) \mathrm{d}\left(\mathrm{T}_{1}\right)$, donde

$$
\begin{aligned}
& \hat{c}\left(\mathrm{~T}_{1}\right)=\mathrm{F} \mathrm{UR}^{\prime} \mathrm{U}^{\prime} \mathrm{R}^{\prime} \mathrm{F}^{\prime} \\
& \mathrm{d}\left(\mathrm{T}_{1}\right)=\hat{\mathrm{d}}\left(\mathrm{T}_{1}\right) \mathrm{Ua}=\mathrm{F} \quad \mathrm{R} \mathrm{U}^{\prime} \mathrm{R}^{\prime} \mathrm{U}^{\prime} \mathrm{F}^{\prime} \mathrm{Ua}
\end{aligned}
$$


Diseñar la configuración $T_{1}$ a partir de la 2 a forma (constructor $\hat{c}\left(\mathrm{~T}_{1}\right)$ ) y orientar el cubo usando la 1 a forma (deconstructor $\mathrm{d}\left(\mathrm{T}_{1}\right)$ ).

Sea $\mathrm{C}_{\mathrm{q}}$ la configuración resultante de ejecutar $\mathbf{q}(=\mathrm{Ua})$ desde el cubo en su estado original. Sucede que $\mathrm{C}_{\mathrm{q}}=\mathrm{C}_{\text {T.o. }}$. (cubo totalmente orientado, pero no totalmente organizado).

\section{$\hat{\mathbf{c}}\left(\mathrm{T}_{1}\right) \mathbf{d}\left(\mathrm{T}_{1}\right) \longrightarrow \mathrm{C}_{\mathrm{T} . \mathrm{O}}$}

Para llevar el cubo a su estado original puede aplicarse el PLL: Ub.

\section{$\hat{c}\left(T_{1}\right) d\left(T_{1}\right) \longrightarrow C_{1}$}

Observación: Los deconstructores de

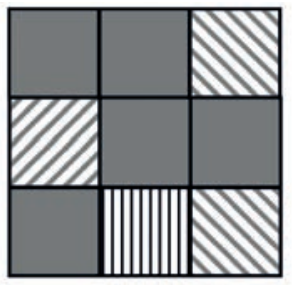
esas dos formas no son semejantes: Si desde el cubo en su estado original se ejecutan uno por uno los deconstructores de ambas formas, se puede notar que llevan a configuraciones no semejantes. Con el deconstructor de la $1^{\text {a }}$ se genera una "Cuasi $Y$ " y con el deconstructor de la $2^{\text {a }}$ se sabe que se genera una "P".

Reforzando esto algebraicamente se tiene:

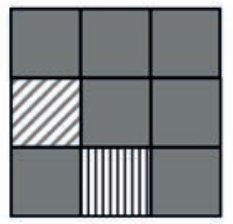

- $\mathrm{d}\left(\mathrm{T}_{1}\right)\left(\hat{\mathrm{d}}\left(\mathrm{T}_{1}\right)\right)^{\prime}=\mathrm{d}\left(\mathrm{T}_{1}\right) \hat{c}\left(\mathrm{~T}_{1}\right)=$ "un pescado de aletas largas "(OLL y no PLL)

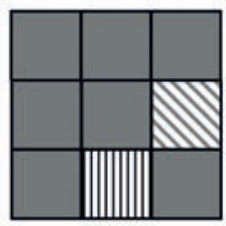

- $\quad \hat{d}\left(\mathrm{~T}_{1}\right) \mathrm{d}\left(\mathrm{T}_{1}\right)^{\prime}=\mathrm{d}\left(\mathrm{T}_{1}\right) \mathrm{c}\left(\mathrm{T}_{1}\right)=$ "un pescado de aletas largas

" (OLL y no PLL)

En consecuencia los algoritmos $d\left(T_{1}\right)$ y $\widehat{d}\left(T_{1}\right)$ NO son semejantes 


\subsection{Nuevos constructor/deconstructor para $T_{2}$.}

\subsubsection{Motivación}

\subsubsection{Dinámica de $\widehat{\mathbf{c}}\left(\mathrm{T}_{2}\right)$ :}

Trabajando con el constructor canónico de $\mathrm{T}_{2}$ (según lo expuesto en 2.1.3) y aplicándolo a la primera figura de la izquierda de más abajo
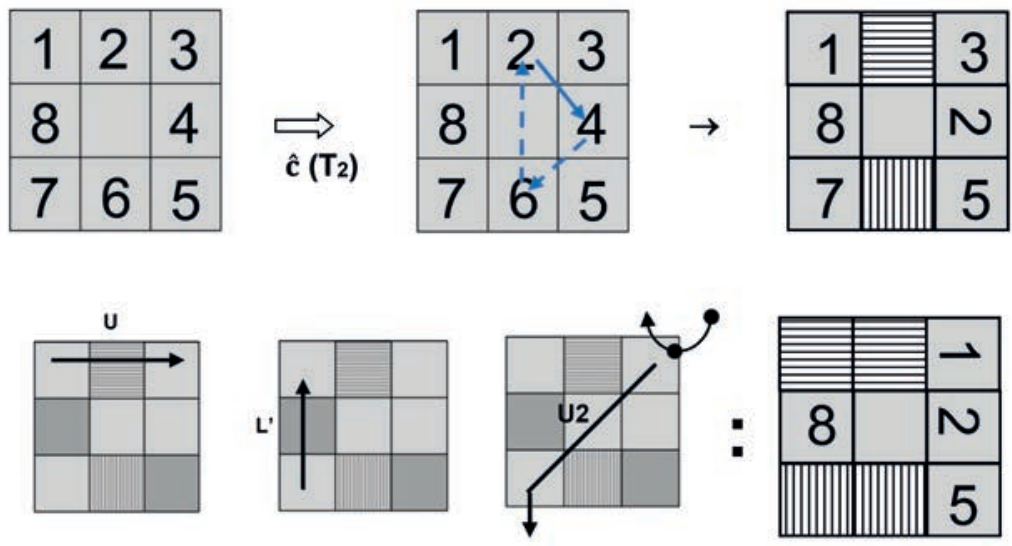

\section{En síntesis:}
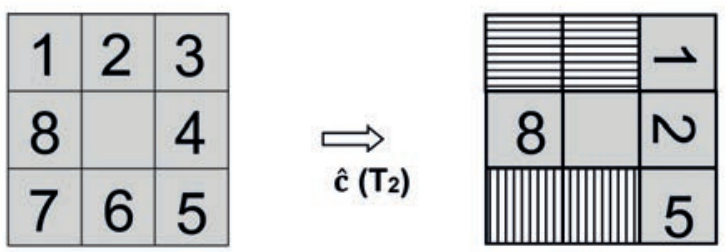

3.8.1.2 Dinámica de $\mathrm{c}\left(\mathrm{T}_{2}\right)=\mathrm{A}_{\mathrm{a}} \widehat{\mathrm{c}}\left(\mathrm{T}_{2}\right)$. Jugando de un modo similar, y aprovechando la síntesis de $(1)$, resulta $\mathrm{c}\left(\mathrm{T}_{2}\right)$ un constructor de $\mathrm{T}_{2}$.

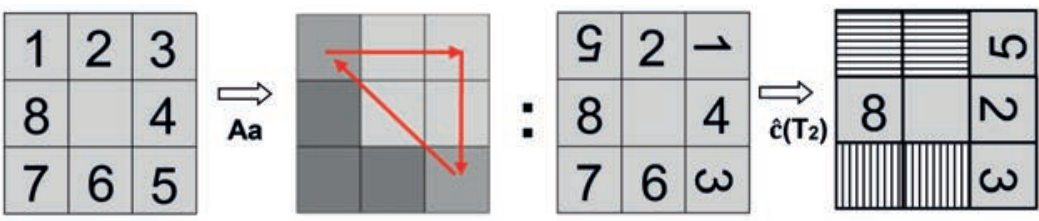


En síntesis:
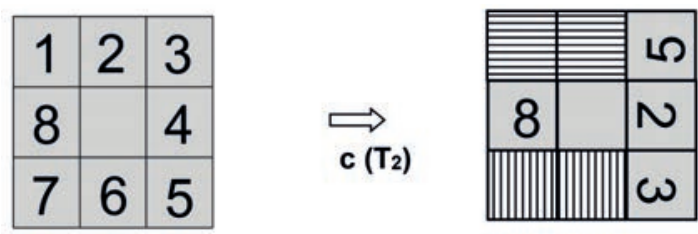

Observación: Los dos algoritmos $\widehat{\mathbf{c}}\left(\mathrm{T}_{2}\right)$ yc $\left(\mathrm{T}_{2}\right)$ realmente construyen a $\mathrm{T}_{2}$, y sus configuraciones son semejantes, pero no congruentes: Con $\widehat{\mathbf{C}}\left(\mathrm{T}_{2}\right)$ el vértice inferior derecho es invariante en tanto que con $\mathrm{c}\left(\mathrm{T}_{2}\right)$ el vértice inferior derecho NO es invariante.

\subsubsection{Dos formas de construir/deconstruir $T_{2}$}

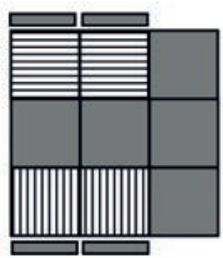

$T_{2}$

\section{$1^{\text {a }}$ Forma}

$c\left(T_{2}\right): A_{a} \hat{\mathbf{c}}\left(T_{2}\right)$ (*)

$d\left(T_{2}\right): R U$ R' U' R' F R F' $\hat{d}\left(T_{2}\right) A_{b}$

$2^{\text {a }}$ Forma ("Canónica")

$\hat{\mathbf{c}}\left(T_{2}\right)$ : F R' F' R U R U' R'

$\hat{d}\left(T_{2}\right): R U R '$ U' R' F R F'

$(*)$

$A_{a} \hat{\mathbf{c}}\left(T_{2}\right)=\left(R^{\prime} F\right.$ R' B2 R F' R R2 B2 R2) (F R' F' R U R U' R'), $y$

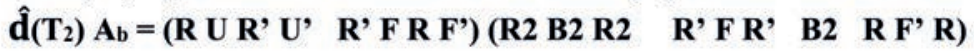

\subsubsection{La no congruencia de las dos formas}

En la configuración de la $2^{\text {a }}$ forma el vértice inferior derecho (de la cara superior) está bien acomodado (bien posicionado y sin torsión), lo que no sucede con la $1^{\text {a }}$ forma.

3.8.4 La semejanza en la configuración de las dos formas Intercambiando constructor/deconstructor.

Sean $\mathbf{m}=\mathbf{c}\left(\mathbf{T}_{2}\right)=$ Aa $\hat{\mathbf{c}}\left(\mathbf{T}_{2}\right)$ y $\mathbf{n}=\hat{\mathbf{c}}\left(\mathbf{T}_{2}\right)=$ F R' F' R U R U' R'.

Por definición 3.2.1. $\mathrm{m} \sim \mathrm{n}\left(\mathrm{m} * \mathrm{n}^{\prime}=\right.$ Aa PLL) . Algo más, al ejecutar los constructores de ambas formas se obtiene la misma configuración por semejanza: 


$$
\widetilde{\boldsymbol{C}_{m}}=\widetilde{\boldsymbol{C}_{n}}
$$

A continuación, se verifica lo anterior también de modo algebraico Intercambiando constructor/deconstructor.

3.8.4.1 Situación 1.1. p: $\mathrm{c}\left(\mathrm{T}_{2}\right) \hat{d}\left(\mathrm{~T}_{2}\right)$, donde

$$
\begin{aligned}
c\left(T_{2}\right)=A_{a} \hat{c}\left(T_{2}\right) & =A_{a} \quad\left(F R^{\prime} F^{\prime} R\right. \text { U R U'R') } \\
\hat{d}\left(T_{2}\right) & =\text { R U R' U' R'FRF' }
\end{aligned}
$$

Diseñar la configuración $I_{1}$ a partir de la $1^{\text {a }}$ forma (constructor $\mathrm{c}\left(\mathrm{T}_{2}\right)$ ) y orientar el cubo usando la $2^{\mathrm{a}}$ forma (deconstructor $\hat{\mathrm{d}}\left(\mathrm{T}_{2}\right)$ ).

Sea $\mathrm{C}_{\mathrm{p}}$ la configuración resultante de ejecutar $\mathbf{p}(=\mathrm{Aa})$ desde el cubo en su estado original. Sucede que $\mathrm{C}_{\mathrm{p}}=\mathrm{C}_{\text {т.o. }}$. (cubo totalmente orientado, pero no totalmente organizado).

$$
\mathbf{c}\left(\mathbf{T}_{2}\right) \hat{\mathbf{d}}\left(\mathrm{T}_{2}\right) \longrightarrow \mathbf{C}_{\mathrm{T} . \mathrm{O}}
$$

Para llevar el cubo a su estado original puede aplicarse el PLL: $\mathbf{A b}$

$$
c\left(T_{2}\right) \hat{d}\left(T_{2}\right) \longrightarrow C_{1}
$$

3.8.4.2 Situación 2. q: $\hat{c}\left(\mathrm{~T}_{2}\right) \mathrm{d}\left(\mathrm{T}_{2}\right)$, donde $\hat{\mathrm{c}}\left(\mathrm{T}_{2}\right)=\mathrm{FR}^{\prime} \mathrm{F}^{\prime} \mathrm{R}$ UR U' R' $\mathrm{d}\left(\mathrm{T}_{2}\right)=\hat{\mathrm{d}}\left(\mathrm{T}_{2}\right) \mathrm{Ab}=\left(\mathrm{R} U \mathrm{R}^{\prime} \mathrm{U}^{\prime} \mathrm{R}^{\prime} \mathrm{F} \mathrm{R} \mathrm{F}^{\prime}\right) \mathrm{Ab}$

Diseñar la configuración $T_{2}$ a partir de la $2^{\text {a }}$ forma (constructor $\hat{\mathrm{c}}\left(\mathrm{T}_{2}\right)$ ) y orientar el cubo usando la 1a forma (deconstructor $\mathrm{d}\left(\mathrm{T}_{2}\right)$ ). 
Sea $\mathrm{C}_{\mathrm{q}}$ la configuración resultante de ejecutar $\mathbf{q}(=\mathrm{Ab})$ desde el cubo en su estado original. Sucede que $\mathrm{C}_{\mathrm{q}}=\mathrm{C}_{\text {т.o. }}$ (cubo totalmente orientado, pero no totalmente organizado).

\section{$\hat{\mathbf{c}}\left(\mathrm{T}_{2}\right) \mathrm{d}\left(\mathrm{T}_{2}\right) \longrightarrow \mathrm{C}_{\mathrm{T} . \mathrm{O}}$}

Para llevar el cubo a su estado original puede aplicarse el PLL: Aa.

\section{$\hat{c}\left(T_{2}\right) d\left(T_{2}\right)$ \\ Aa}

Observación: Los deconstructores de esas dos formas no son semejantes: Si desde el cubo en su estado original se ejecutan uno por uno los deconstructores de ambas formas, se puede notar que llevan a configuraciones no semejantes. Con el deconstructor de la $1^{\text {a }}$ se genera una "Cuasi Y" y con el deconstructor de la $2^{\text {a }}$ se sabe que se genera una "Cometa". Reforzando esto algebraicamente se tiene:

- $\mathrm{d}\left(\mathrm{T}_{2}\right) \hat{\mathbf{d}}\left(\mathbf{T}_{2}\right)$ " $=\mathrm{d}\left(\mathrm{T}_{2}\right) \hat{\mathbf{c}}\left(\mathbf{T}_{2}\right)=$ "Pajarita" (OLL y no PLL)

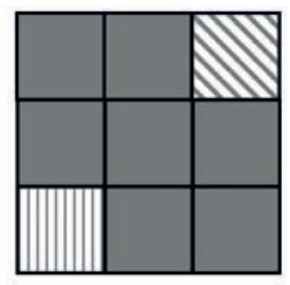

Pajarita

- $\quad \hat{\mathbf{d}}\left(\mathbf{T}_{2}\right) \mathrm{d}\left(\mathrm{T}_{2}\right)$ " = $\hat{\mathbf{d}}\left(\mathbf{T}_{2}\right) \mathrm{c}\left(\mathrm{T}_{2}\right)=$ "Botella" (OLL y no PLL)

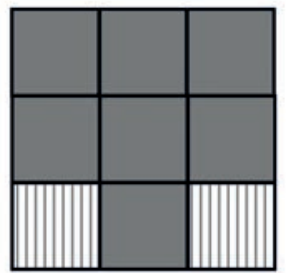

\section{Botella}

En consecuencia, $d\left(\mathrm{~T}_{2}\right)$ y $\hat{\mathbf{d}}\left(\mathrm{T}_{2}\right)$ son algoritmos NO semejantes 


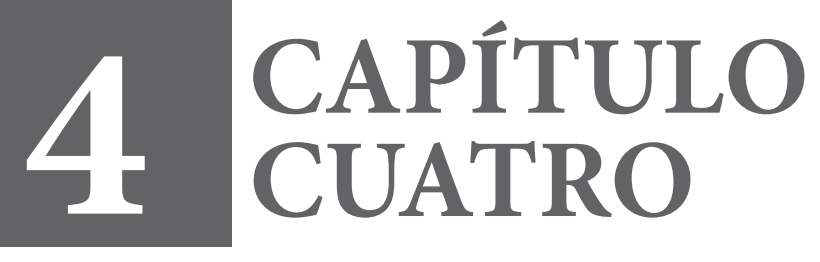




\section{Las potencias dos y tres con $\mathrm{T}_{1}$ : “ $\mathrm{I}_{1}$ " $\mathrm{y}$ "Cruz"}

\section{Los PLL J, N y V}

Este par de OLL son sencillos de memorizar en sus formas canónicas, y en particular la "I" resulta sumamente útil en la deconstrucción de otros OLL, que en su configuración poseen una "mini J" (denominadas "Mini J" ampliadas, a tratar más adelante en este libro). Se observa que un tratamiento adecuado de este par de OLL, lleva a nuevos PLL como son las dos J, las dos $\mathrm{N}$ y la V.

\section{1 $\mathrm{I}_{1}$ : La Primera "I" y dos de sus constructores}

\subsubsection{Motivación}

$\mathrm{I}_{1}=\mathrm{d}\left(\mathrm{T}_{1}\right) \mathrm{d}\left(\mathrm{T}_{2}\right)$. Se aplica el deconstructor canónico de $\mathrm{T}_{2}$ (según lo expuesto en 2.2.2) a la $\mathrm{P}_{1}$ de más abajo $\left(=\mathrm{d}\left(\mathrm{T}_{1}\right)\right.$ )
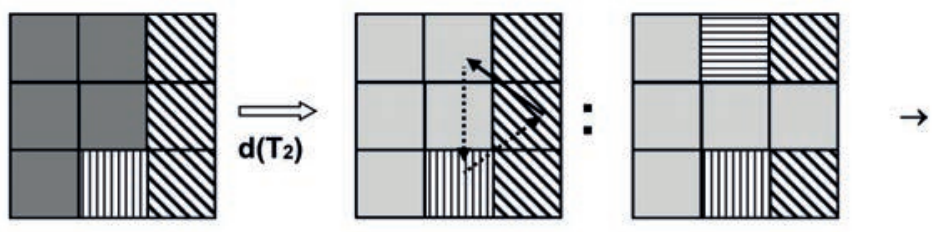

$\mathbf{P}_{1}$
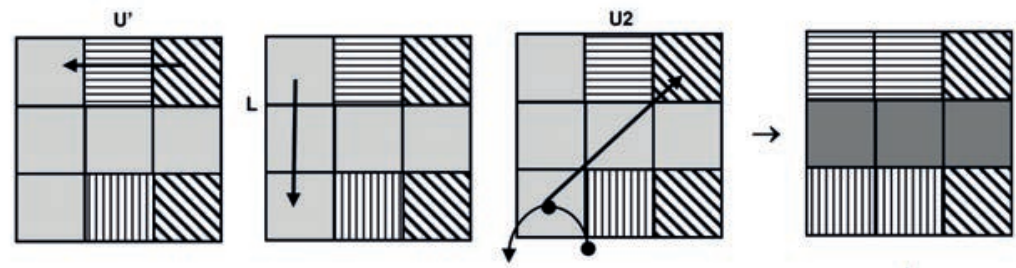

$l_{1}$ 
4.1.2 Dos formas de construir/deconstruir $\mathrm{I}_{1}$

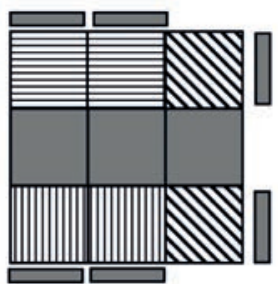

$I_{1}$ $1^{\text {a }}$ Forma

$c\left(I_{1}\right): d\left(T_{1}\right) d\left(T_{2}\right)$

$d\left(I_{1}\right): c\left(T_{2}\right) c\left(T_{1}\right)$

$2^{\text {a }}$ Forma

$\hat{\mathbf{c}}\left(\mathrm{l}_{1}\right): \mathrm{d}\left(\mathrm{T}_{1}\right) \mathbf{2}=\mathbf{F}\left(\mathbf{R} \cup R^{\prime} \mathbf{U}^{\prime}\right) 2 \mathrm{~F}^{\prime}$

$\hat{d}\left(I_{1}\right): c\left(T_{1}\right) 2=F\left(U^{\prime} R^{\prime}\right) 2$ F'

(*) $\mathbf{d}\left(\mathbf{T}_{1}\right) \mathbf{d}\left(\mathbf{T}_{2}\right)=($ F R U R' U' F') ( R U R' U' R' F R F')

$c\left(T_{2}\right) c\left(T_{1}\right)=(F$ R' F' R U R U' R') (F U R U' R' F')

\subsubsection{La no congruencia de las dos formas}

Una diferencia radica en los vértices de la cara superior: Con la $2^{\text {a }}$ forma todos los vértices quedan bien posicionados, cosa que no se da con la $1^{\text {a }}$ forma.

\subsubsection{La semejanza en la configuración de las dos formas}

Al ejecutar los constructores de ambas formas se obtiene la misma configuración por semejanza:

$$
\widetilde{\mathbf{C}_{\mathbf{p}}}=\widetilde{\mathbf{C}_{\mathbf{q}}}
$$

siendo $\mathrm{p}=\mathrm{c}\left(\mathrm{I}_{1}\right)=\mathbf{d}\left(\mathbf{T}_{1}\right) \mathbf{d}\left(\mathbf{T}_{2}\right) \quad \mathbf{y} \quad \mathrm{q}=\hat{\mathbf{c}}\left(\mathrm{I}_{1}\right)=\mathbf{d}\left(\mathbf{T}_{1}\right) \mathbf{2}$

A continuación, se verificará lo anterior también de modo algebraico Intercambiando constructor/deconstructor.

\subsubsection{Situación 1 p: $\mathbf{c}\left(\mathrm{I}_{1}\right) \hat{\mathrm{d}}\left(\mathrm{I}_{1}\right)=\hat{\mathrm{d}}\left(\mathrm{T}_{1}\right) \hat{\mathrm{d}}\left(\mathrm{T}_{2}\right) \hat{\mathbf{c}}\left(\mathrm{T}_{1}\right) \mathbf{2}$}

Donde:

$$
\begin{aligned}
& \hat{\mathbf{d}}\left(\mathbf{T}_{1}\right)=\text { F R U R' U' F' } \hat{\mathbf{d}}\left(\mathbf{T}_{2}\right)=\text { RU R' U' R' FRF' } \\
& \hat{\mathbf{c}}\left(\mathbf{T}_{1}\right) 2=\mathrm{F}\left(\mathrm{UR} \mathrm{U}^{\prime} \mathrm{R}^{\prime}\right) 2 \mathrm{~F}^{\prime} .
\end{aligned}
$$


Diseñar la configuración a partir de la $1^{\text {a }}$ forma (constructor $\mathbf{c}\left(\mathbf{I}_{1}\right)$ ) y orientar el cubo usando la $2^{\text {a }}$ forma (deconstructor $\hat{\mathbf{d}}\left(\mathbf{I}_{1}\right)$ ).

Al ejecutar ese algoritmo p experimentalmente se obtiene el cubo totalmente orientado, pero no en su estado original.

Para llevarlo a ese estado debe ejecutarse el PLL:

\section{U'Aa U}

donde Aa: R' F R' B2 R F' R $\quad$ R2 B2 R2

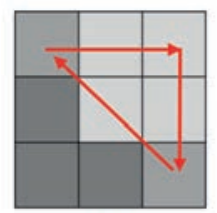

$\mathrm{Aa}$

\title{
4.1.4.2 Situación 2: "Inversa de la situación 1"
}

$$
q: \hat{c}\left(\mathbf{I}_{1}\right) \mathbf{d}\left(\mathbf{I}_{1}\right)=\mathbf{d}\left(\mathbf{T}_{1}\right) \mathbf{2} \quad \mathbf{c}\left(\mathbf{T}_{2}\right) \mathbf{c}\left(\mathbf{T}_{1}\right),
$$

Donde:

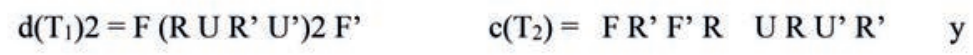

$c\left(T_{1}\right)=$ F U R U' R' F'

Diseñar la configuración "I" a partir de la $2^{\text {a }}$ forma (constructor $\hat{\mathbf{c}}\left(\mathbf{I}_{\mathbf{1}}\right)$ ) y orientar el cubo usando la $1^{\text {a }}$ forma (deconstructor $\mathbf{d}\left(\mathbf{I}_{1}\right)$ ). Al ejecutar ese algoritmo $\mathbf{q}$ experimentalmente se obtiene el cubo totalmente orientado, pero no en su estado original.

Para llevarlo a ese estado debe ejecutarse el PLL:

\author{
U'Ab U \\ donde Ab: R2 B2 R2 R' F R' B2 R F' R
}

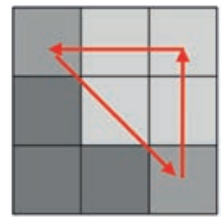

Ab 


\subsubsection{Dinámica del $\widehat{\mathrm{C}}\left(\mathrm{I}_{1}\right)$ en la capa superior}
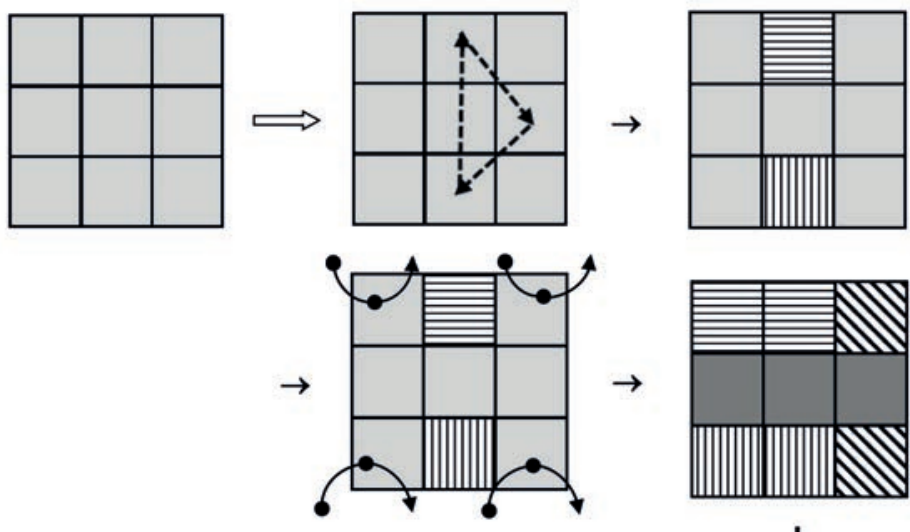

I.

4.1.5.1 Acción de $\widehat{\mathrm{c}}\left(\mathrm{I}_{1}\right)$ sobre sus aristas iniciales $\widehat{\mathrm{c}}\left(\mathrm{I}_{1}\right)$ realiza, al igual que $\mathrm{c}\left(\mathrm{T}_{1}\right)$, una permutación en sentido horario de tres de las aristas distintas de la izquierda (permanece invariante), provocando permutaciones en los stickers de algunas de estas. De modo preciso:

- La arista superior pasa a ser la arista derecha;

- La arista derecha pasa a ser la inferior y sus stickers permutan;

- La arista inferior pasa a ser la superior y sus stickers permutan.

4.1.5.2 Acción de $\widehat{c}\left(\mathrm{I}_{1}\right)$ sobre sus vértices iniciales.

- Los vértices frontales (inferior izquierdo e inferior derecho) reciben una torsión "horaria" de tres giros (1.6.3.3).

- Los vértices de atrás (superior izquierdo y superior derecho) reciben una torsión "antihoraria" de tres giros (1.6.3.3). 
4.2 Nuevos constructor/deconstructor de $\mathrm{I}_{1}$. Los PLL "J"

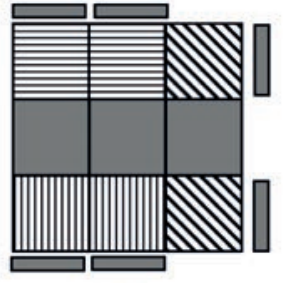

$I_{1}$
19 Forma ("Nueva"):

$c\left(l_{1}\right)=d\left(T_{1}\right)^{s} d\left(T_{2}\right)^{s} U_{2}$

$d\left(l_{1}\right)=U 2 c\left(T_{2}\right)^{s} c\left(T_{1}\right)^{s}$

$2^{\text {a }}$ Forma ("Canónica"):

$\hat{c}\left(\mathbf{l}_{1}\right): F\left(R \cup R^{\prime} U^{\prime}\right) 2 F^{\prime}$

d (li): F (U R U' R')2 F'

(*) $d\left(T_{1}\right)^{S} d\left(T_{2}\right)^{S}=\left(F^{\prime} L^{\prime} U^{\prime} L U^{\prime} F\right.$ ) ( L' U' L U L F' L' F) $c\left(T_{2}\right)^{S} \mathbf{c}\left(T_{1}\right)^{S}=\left(F^{\prime} L\right.$ F L' U' L' U L) (F' U' L' U L F)

4.2.1 La no congruencia de las dos formas

En la configuración de la $2^{\text {a }}$ forma el vértice inferior derecho (de la cara superior) está bien posicionado, lo que no sucede con la $1^{\text {a }}$ forma.

\subsubsection{La semejanza en la configuración de las dos formas}

Intercambiando constructor/deconstructor. La permutación "J ${ }_{D}$ ".

Sean $\mathbf{m}=\mathbf{c}\left(\mathbf{m I} \mathbf{I}_{1}\right): \mathbf{d}\left(\mathbf{T}_{1}\right)^{\mathbf{s}} \mathbf{d}\left(\mathbf{T}_{2}\right)^{\mathbf{s}} \mathrm{U} 2$ y $\mathbf{n}=\hat{\mathbf{c}}\left(\mathbf{m} \mathbf{I}_{\mathbf{1}}\right): F \quad\left(R \mathrm{R}^{\prime} \mathrm{U}^{\prime}\right) 2$ F'. Al ejecutar los constructores de ambas formas se obtiene la misma configuración por semejanza:

$$
\widetilde{\mathbf{C}_{\mathbf{m}}}=\widetilde{\mathbf{C}_{\mathbf{n}}}
$$

A continuación, se verificará lo anterior también de modo algebraico Intercambiando constructor/deconstructor.

4.2.2.1 Situación 1. p: $\mathbf{c}\left(\mathbf{I}_{1}\right) \mathbf{d}\left(\mathbf{I}_{1}\right)$, donde

$$
\begin{gathered}
\mathrm{c}\left(\mathrm{I}_{1}\right)=\mathrm{d}\left(\mathrm{T}_{1}\right)^{\mathrm{S}} \mathrm{d}\left(\mathrm{T}_{2}\right)^{\mathrm{S}} \mathrm{U} 2=\left(\mathrm{F}^{\prime} \mathrm{L}^{\prime} \mathrm{U}^{\prime}\right. \text { LUF) (L'U'LU LF'L'F) U2 } \\
\mathrm{d}\left(\mathrm{I}_{1}\right)=\mathrm{F}(\mathrm{UR} \text { U'R')2 F' }
\end{gathered}
$$

Diseñar la configuración $I_{1}$ a partir de la $1^{\text {a }}$ forma (constructor $\mathrm{c}\left(\mathrm{I}_{1}\right)$ ) y orientar el cubo usando la $2^{\text {a }}$ forma (deconstructor $\left.\hat{\mathrm{d}}\left(\mathrm{I}_{1}\right)\right)$. 
Sea $C_{p}$ la configuración resultante de ejecutar $\mathbf{p}$ desde el cubo en su estado original. Sucede que $\mathrm{C}_{\mathrm{p}}=\mathrm{C}_{\text {т.о. }}$ (cubo totalmente orientado, pero no totalmente organizado).

$$
\mathbf{C}\left(\mathrm{I}_{1}\right) \hat{\mathbf{d}}\left(\mathrm{I}_{1}\right) \longrightarrow \mathrm{C}_{\mathrm{T} .0}
$$

Para llevar el cubo a su estado original puede aplicarse el PLL:

$$
\mathrm{U} 2 \mathrm{~J}_{\mathrm{D}} \mathrm{U}^{\prime}
$$

donde $\mathrm{J}_{\mathrm{D}}$ : Ua U Aa U2

$$
\hat{c}\left(I_{1}\right) d\left(I_{1}\right) \underset{U 2 ~ J_{D} U}{\longrightarrow} C_{1}
$$

\subsubsection{Situación 2. $\hat{\mathbf{c}}\left(\mathbf{I}_{1}\right) \mathrm{d}\left(\mathrm{I}_{1}\right)$, donde \\ $\widehat{\mathrm{c}}\left(\mathrm{I}_{1}\right)=\mathrm{F}(\mathrm{R}$ U R' U') 2 F' \\ $\mathrm{d}\left(\mathrm{I}_{1}\right)=\mathrm{U} 2 \mathrm{c}\left(\mathrm{T}_{2}\right)^{\mathrm{S}} \mathrm{c}\left(\mathrm{T}_{1}\right)^{\mathrm{S}}=\mathrm{U} 2$ (F'LF L' U'L'UL) (F'U'L' ULF)}

Diseñar la configuración $I_{1}$ a partir de la $2^{\text {a }}$ forma (constructor $\left.\hat{c}\left(\mathrm{I}_{1}\right)\right)$ y orientar el cubo usando la $1^{\text {a }}$ forma (deconstructor $\mathrm{d}\left(\mathrm{I}_{1}\right)$ ).

Sea $\mathrm{C}_{\mathrm{q}}$ la configuración resultante de ejecutar $\mathbf{q}$ desde el cubo en su estado original. Sucede que $\mathrm{C}_{\mathrm{q}}=\mathrm{C}_{\text {T.o. }}$ (cubo totalmente orientado, pero no totalmente organizado).

$$
\mathrm{c}\left(\mathrm{I}_{1}\right) \hat{\mathbf{d}}\left(\mathrm{I}_{1}\right) \longrightarrow \mathrm{C}_{\mathrm{T} .0}
$$

Para llevar el cubo a su estado original puede aplicar el PLL: $\mathbf{U} \mathbf{J}_{\mathbf{D}} \mathbf{U}$ 2.

$$
\hat{c}\left(I_{1}\right) d\left(I_{1}\right) \underset{U J_{D} U 2}{\longrightarrow} C_{1}
$$

con $J_{\mathrm{D}}: \quad \mathrm{UaU} \mathrm{Ua} \mathbf{2}$ 


\subsection{Las fascinantes permutaciones " $\mathrm{J}_{\mathrm{D}}$ " $\mathrm{y}$ “ $\mathrm{J}_{\mathrm{I}}$ "}

Innegablemente es uno de los PLL más agradables para memorizar (de pronto no es el más fácil en ese sentido). Junto con la otra $\mathrm{J}\left(\mathrm{J}_{1}\right)$ se denominan "Algoritmos fieles": una vez practicada y observada con cuidado su construcción, es muy difícil que se olviden en el futuro.

Objetivo de las "J": Estas permutaciones lo que hacen es intercambiar dos vértices contiguos y a la vez intercambia la arista comprendida entre estos y su arista contigua.

\subsubsection{La permutación $\mathrm{J}_{\mathrm{D}}$}

- Motivación: Ua U Aa U2 = J
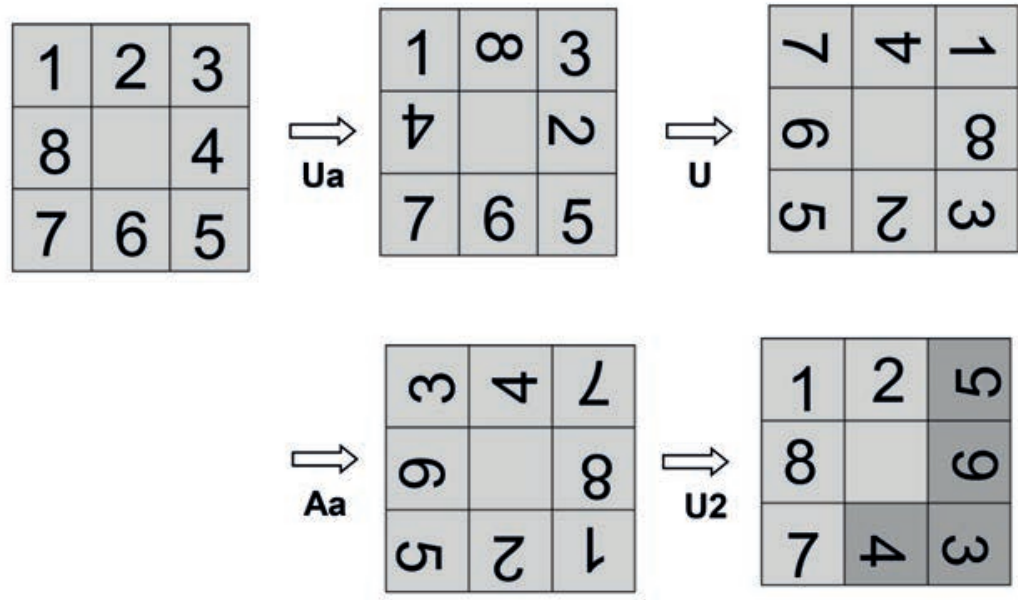

En síntesis:
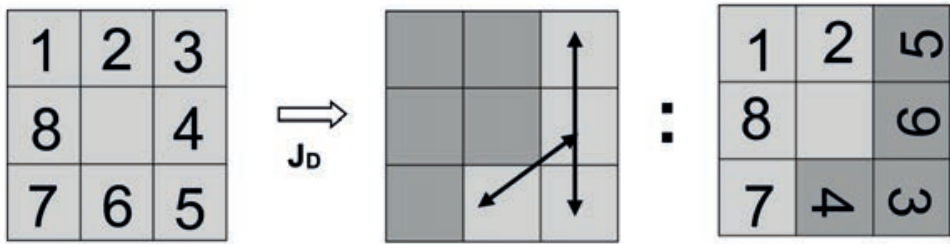
- Forma canónica del PLL “ $\mathrm{J}_{\mathrm{D}}$ ” (Permuta simultáneamente los dos vértices del lado

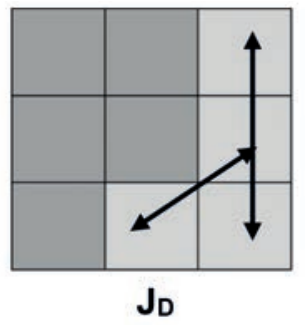
derecho y las aristas inferior y derecha).

\section{B U’ B L2 F’ D F’ D’ F2 L2 B2}

Con el cubo en su estado original y ejecutando "J" surge esa configuración. Una vez hecho " $J_{D}$ " para volver al cubo en su estado original debe repetir tal ejecución.

4.3.2 $\mathrm{La}_{\mathrm{I}}$ : La simétrica de $\mathrm{J}_{\mathrm{D}}$

- Motivación: Ub U' Aa ${ }^{\mathrm{s}} \mathrm{U} 2=\mathrm{J}_{\mathrm{I}}$
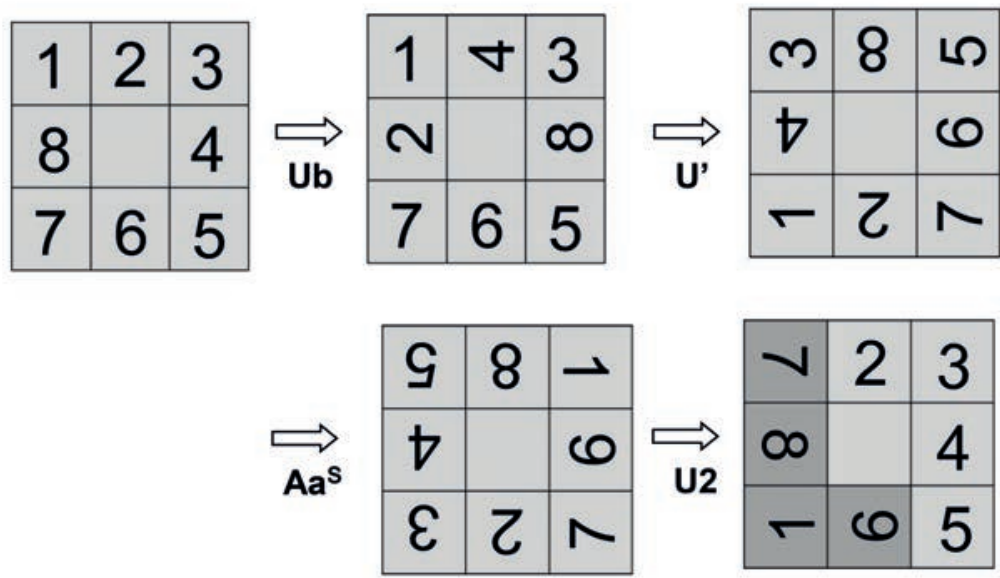

En síntesis:
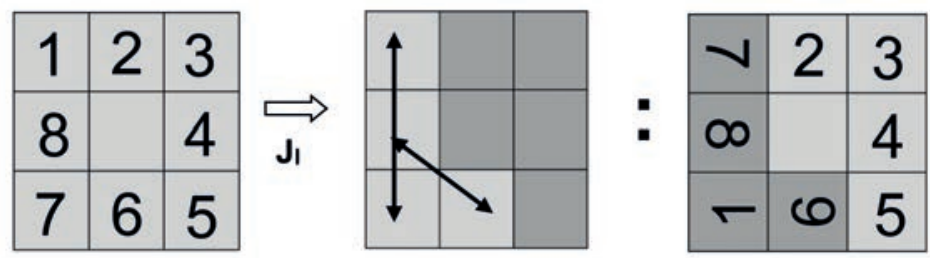
- Forma canónica del PLL “J” (Permuta simultáneamente los dos vértices del lado izquierdo y las aristas inferior e izquierda).

\section{B' U B' R2 F D' F D F2 R2 B2}

Con el cubo en su estado original y ejecutando "J" surge esa configuración. Una vez hecho "J." para volver al cubo en su estado original debe repetir tal ejecución.

\subsubsection{Configuración para aplicar los PLL J}

- Para realizar esta configuración únicamente se tendrá en cuenta la capa superior, especialmente en los lados de arriba de las caras laterales (las otras dos capas están ya organizadas y no nos interesan mucho en este momento).

- Los tres stickers de una de las caras laterales (lado de arriba) tienen el mismo color, en tanto que en las otras caras no sucede eso, pero en cada una de ellas se observan bloques de dos sticker juntos del mismo color mientras que el tercer sticker siempre queda a la derecha o a la izquierda de dicho bloque en c/u de esos lados. Llamamos a ese tercer sticker "sticker referencial para la J"

\section{Ejecución y determinación del "J" adecuado:}

a) Si "sticker referencial para la J" queda ubicado a la izquierda (los bloques de dos stickers juntos quedan a la derecha), colocamos el lado de los 3 stickers del mismo color en la cara izquierda y aplicamos “ $J_{\mathrm{D}}$ ”. De lo contrario,

b) Si "sticker referencial para la J" queda ubicado a la derecha (los bloques de dos stickers juntos quedan a la izquierda), colocamos el lado de los 3 stickers del mismo color en la cara derecha y aplicamos "J".

Al final con movimientos $\mathrm{U}$ se organiza totalmente el cubo. 


\subsection{Las Permutaciones "N"}

Habiendo conocido ya las permutaciones "J", nos disponemos abordar dos interesantes permutaciones que surgen al interactuar las "J" con las rotaciones U de la capa superior. Son las permutaciones que permiten intercambiar dos vértices opuestos entre si y a la vez dos aristas opuestas.

\subsubsection{La permutación $\mathrm{N}_{\mathrm{D}}$}

- Motivación: $\mathrm{UJ}_{\mathrm{D}} \mathrm{U} 2 \mathrm{~J}_{\mathrm{D}}=\mathrm{N}_{\mathrm{D}}$
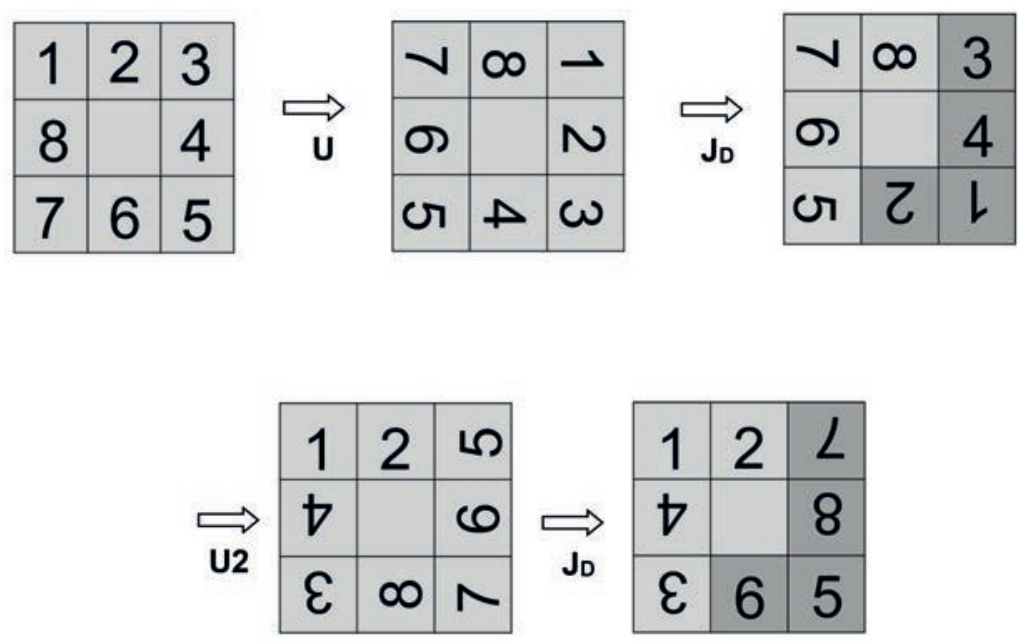

En síntesis:
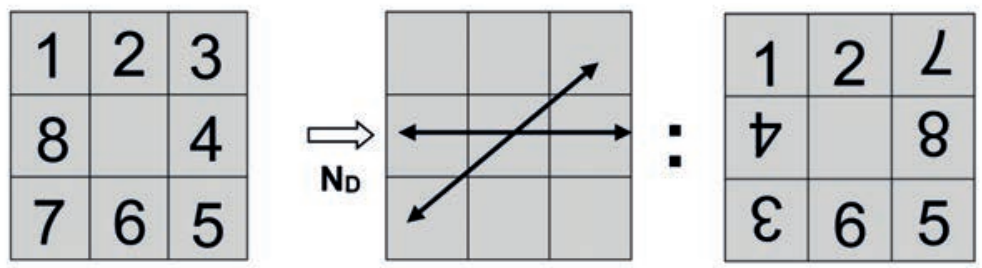

- Forma canónica del PLL “ $\mathrm{N}_{\mathrm{D}}$ ”

(RU'L U2 R'UL')2 U' 
Con el cubo en su estado original y ejecutando " $\mathbf{N}_{\mathrm{D}}$ " surge esa configuración. Una vez hecho " $\mathbf{N}_{\mathrm{D}}$ " para volver al cubo en su estado original debe repetir tal ejecución.

Con $\mathbf{N}_{\mathbf{D}}$ se conmutan los vértices de la diagonal principal y también las aristas derecha e izquierda de la cara superior.

\subsubsection{La permutación $\mathrm{N}_{\mathrm{I}}$ : La simétrica de $\mathrm{N}_{\mathrm{D}}$.}

- Motivación: U’J, U2 J $=N_{1}$
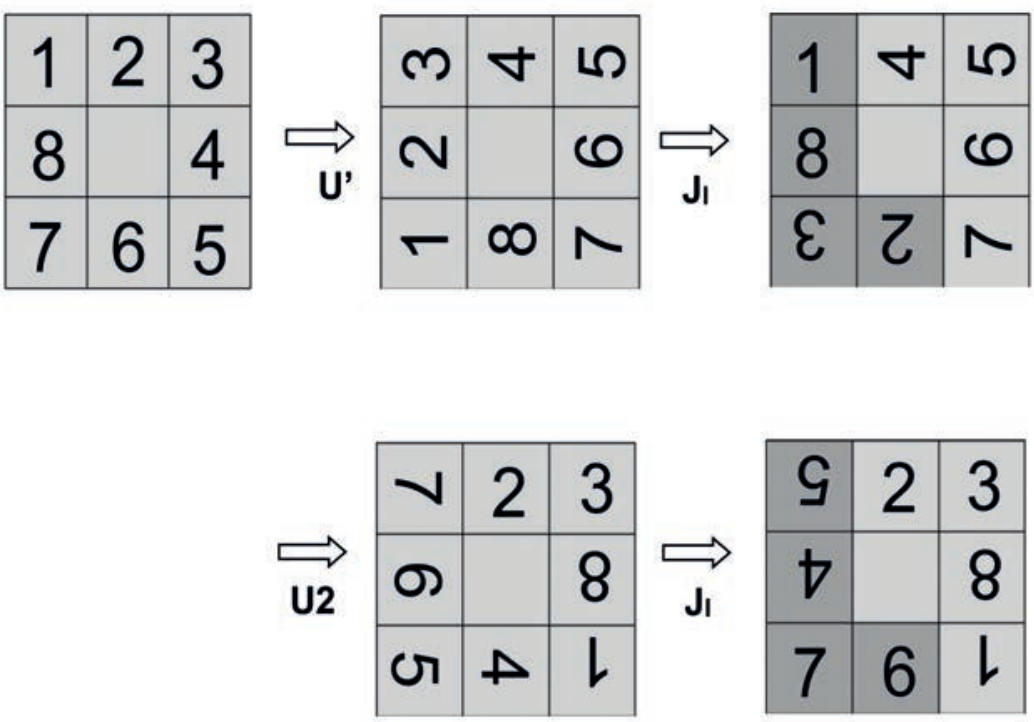

En síntesis:
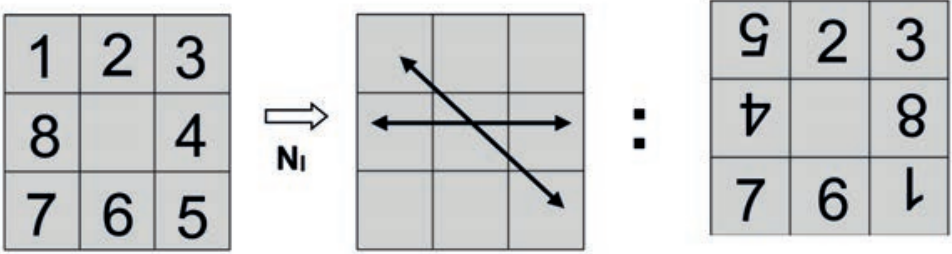

- Forma canónica del PLL “ $\mathrm{N}_{\mathrm{I}}$ ”

(L'UR' U2 LU'R)2 U 


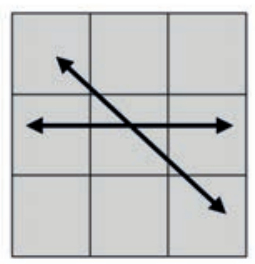

Con el cubo en su estado original y ejecutando " $N_{I}$ " surge esa configuración. Una vez hecho " $\mathbf{N}_{\mathrm{I}}$ " para volver al cubo en su estado original debe repetir tal ejecución.

Con $\mathbf{N}_{\mathrm{I}}$ se conmutan los vértices de la diagonal NO principal y también las aristas derecha e izquierda de la cara superior.

\subsubsection{Configuración para aplicar los PLL N}

- Para realizar esta configuración únicamente se tendrá en cuenta la capa superior, especialmente en los lados de arriba de las caras laterales (las otras dos capas están ya organizadas y no nos interesan mucho en este momento).

- En cada uno de esos lados se observa un bloque de dos stickers juntos del mismo color ${ }^{20}$, mientras que el tercer sticker siempre queda a la derecha o a la izquierda de dicho bloque en c/u de esos lados. Llamamos a ese tercer sticker "sticker referencial para la N"

\section{Ejecución y determinación del "N" adecuado:}

a) Si "sticker referencial para la N" queda ubicado a la izquierda (los bloques de dos stickers juntos quedan a la derecha), aplicamos " $\mathrm{N}_{\mathrm{D}}$ ". De lo contrario,

b) Si "sticker referencial para la N" queda ubicado a la derecha (los bloques de dos stickers juntos quedan a la izquierda), aplicamos “ $\mathrm{N}_{\mathrm{I}}$ ".

Al final, con movimientos $U$, se organiza totalmente el cubo.

20 El lector puede comprobar que hay dos vértices diagonales bien acomodados (los de la diagonal no principal) y dos aristas opuestas bien acomodadas (la superior y la inferior). 
4.5 $\mathrm{I}_{1}^{\mathrm{S}}$ : La simétrica de "I $\mathrm{I}_{1}$ ” dos de sus constructores

4.5.,1 Motivación: $I_{1}{ }^{S}=d\left(T_{1}\right)^{s} d\left(T_{2}\right)^{s}$

$\mathrm{I}_{1}^{\mathrm{S}}=\mathbf{d}\left(\mathrm{T}_{1}\right)^{\mathrm{S}} \mathbf{c}\left[\left(\mathrm{T}_{2}^{\mathrm{S}}\right)^{\prime}\right]$. Apliquemos el constructor canónico de $\left(\mathrm{T}_{2}{ }^{\mathrm{S}}\right)^{\prime}$ (según lo expuesto en 2.4.2) a la $\mathbf{q}_{\mathbf{1}}$ de más abajo (= $\left.\mathrm{d}\left(\mathrm{T}_{1}\right)^{\mathrm{S}}\right)$

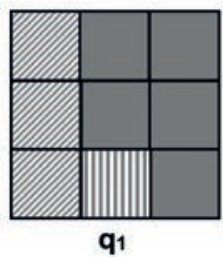

U
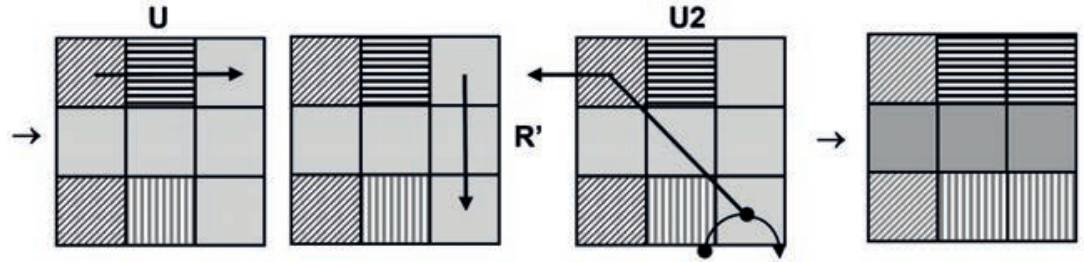

$\mathrm{I}_{1}^{\mathrm{S}}$

\subsubsection{Dos formas de construir/deconstruir $\mathrm{I}_{1}^{\mathrm{s}}$}
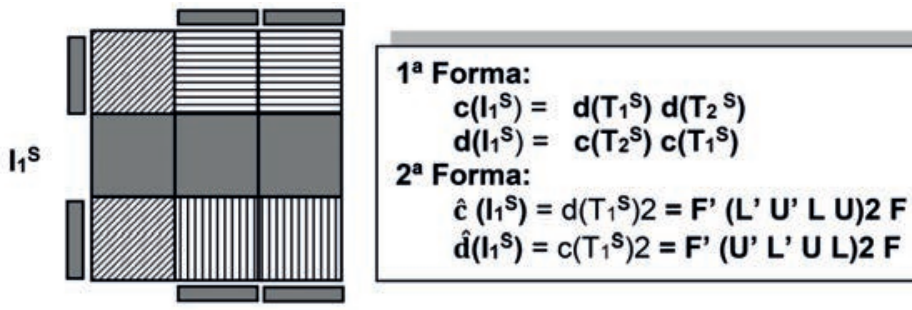
(*) $\quad d\left(T_{1}{ }^{S}\right) d\left(T_{2} s^{S}\right)=\left(F^{\prime} L^{\prime} U^{\prime}\right.$ L U F) ( L' U' L U L F' L' F )
$c\left(T_{2}{ }^{S}\right) c\left(T_{1}{ }^{S}\right)=\left(F^{\prime} L F^{\prime} L^{\prime} \quad U^{\prime} L^{\prime}\right.$ U L) (F' U' L' U L F)

\subsubsection{La no congruencia de las dos formas}

Una diferencia radica en los vértices de la cara superior: Con la $2^{\mathrm{a}}$ forma todos los vértices quedan bien posicionados, cosa que no se da con la $1^{\text {a }}$ forma. 
4.5.4 La semejanza en la configuración de las dos formas

$\mathrm{Al}$ ejecutar los constructores de ambas formas se obtiene la misma configuración por semejanza:

$$
\widetilde{\mathbf{C}_{\mathbf{p}}}=\widetilde{\mathbf{C}_{\mathbf{q}}}
$$

con $\mathrm{p}=\mathrm{c}\left(\mathrm{I}_{1}^{\mathrm{S}}\right)=\mathrm{d}\left(\mathrm{T}_{1}{ }^{\mathrm{S}}\right) \mathrm{d}\left(\mathrm{T}_{2}{ }^{\mathrm{S}}\right)$ y $\mathrm{q}=\hat{\mathrm{c}}\left(\mathrm{I}_{1}^{\mathrm{S}}\right)=\mathrm{c}\left(\mathrm{T}_{1}\right) 2=\mathrm{F}^{\prime}\left(\mathrm{L}^{\prime} \mathrm{U}^{\prime} \mathrm{L} \mathrm{U}\right) 2 \mathrm{~F}$

A continuación, se verificará lo anterior también de modo algebraico intercambiando constructor/deconstructor.

4.5.4.1 Situación 1. p: $\mathrm{e}\left(\mathrm{I}_{1}^{\mathrm{S}}\right) \mathrm{d}\left(\mathrm{d}_{1}^{\mathrm{S}}\right)=\mathrm{d}\left(\mathrm{T}_{1}{ }^{\mathrm{S}}\right) \mathrm{d}\left(\mathrm{T}_{2}^{\mathrm{S}}\right) \mathrm{c}\left(\mathrm{T}_{1}{ }^{\mathrm{s}}\right) 2$

donde

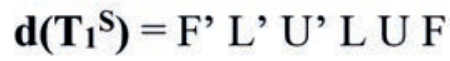

$$
\begin{aligned}
& \mathbf{d}\left(\mathbf{T}_{2}{ }^{\mathbf{S}}\right)=\text { L'U' L U }^{\prime} \text { L F' L'F } \quad \mathrm{y} \\
& \hat{\mathbf{c}}\left(\mathbf{T}_{1}{ }^{\mathbf{S}}\right) 2=\mathrm{F}^{\prime}\left(\mathrm{U}^{\prime} \mathrm{L}^{\prime} \mathrm{U} \mathrm{L}\right) 2 \mathrm{~F}
\end{aligned}
$$

Diseñar la configuración a partir de la $1^{\text {a }}$ forma (constructor $\mathbf{c}\left(\mathbf{I}^{\mathrm{S}}\right)$ ) v orientar el cubo usando la $2^{\mathrm{a}}$ forma (deconstructor $\hat{\mathbf{d}}\left(\mathbf{I}_{1} \mathbf{S}\right)$ ). Al ejecutar ese algoritmo p experimentalmente obtenemos el cubo totalmente orientado, pero no en su estado original. Para llevarlo a ese estado debe ejecutarse el PLL:

\section{U2 Ab U2}

donde $\mathrm{Ab}=\mathrm{R} 2 \mathrm{~B} 2 \mathrm{R} 2 \quad \mathrm{R}^{\prime} \mathrm{F} \mathrm{R}^{\prime} \quad \mathrm{B} 2 \quad \mathrm{R} \mathrm{F}^{\prime} \mathrm{R}, 2.6 .2 .2$.

\subsubsection{Situación 2: "Inversa de la situación 1"}

$$
\text { q: } \hat{\mathbf{c}}\left(\mathrm{I}_{1}^{\mathrm{S}}\right) \mathbf{d}\left(\mathrm{I}_{1}^{\mathrm{S}}\right)=\mathbf{d}\left(\mathrm{T}_{1}^{\mathrm{S}}\right) 2 \quad \mathrm{c}\left(\mathrm{T}_{2}^{\mathrm{S}}\right) \mathbf{c}\left(\mathrm{T}_{1}^{\mathrm{S}}\right),
$$

donde

$$
\begin{aligned}
& \mathrm{d}\left(\mathrm{T}_{1}^{\mathrm{S}}\right) 2=\mathrm{F}^{\prime}\left(\mathrm{L}^{\prime} \mathrm{U}^{\prime} \mathrm{L} \text { U) } 2 \mathrm{~F}\right. \\
& c\left(T_{2}{ }^{S}\right)=F^{\prime} L F L \quad U^{\prime} L^{\prime} U L \quad y \\
& c\left(T_{1}^{S}\right)=F^{\prime} U^{\prime} L^{\prime} U L \text { F. }
\end{aligned}
$$


Diseñar la configuración " $\mathrm{I}$ " a partir de la $2^{\mathrm{a}}$ forma (constructor $\hat{\mathbf{c}}\left(\mathbf{I}_{1} \mathbf{S}\right)$ ) y orientar el cubo usando la $1^{\text {a }}$ forma (deconstructor $\mathbf{d}\left(\mathbf{I}_{1}^{s}\right)$ ). Al ejecutar ese algoritmo $\mathbf{q}$ experimentalmente obtenemos el cubo totalmente orientado, pero no en su estado original. Para llevarlo a ese estado ejecutar el PLL:

\section{U2 Aa U2}

donde Aa: R' F R' B2 R F' R R2 B2 R2, 2.6.1.2.

\subsubsection{Dinámica del $\hat{c}\left(I_{1}{ }^{S}\right)$ en la capa superior.}
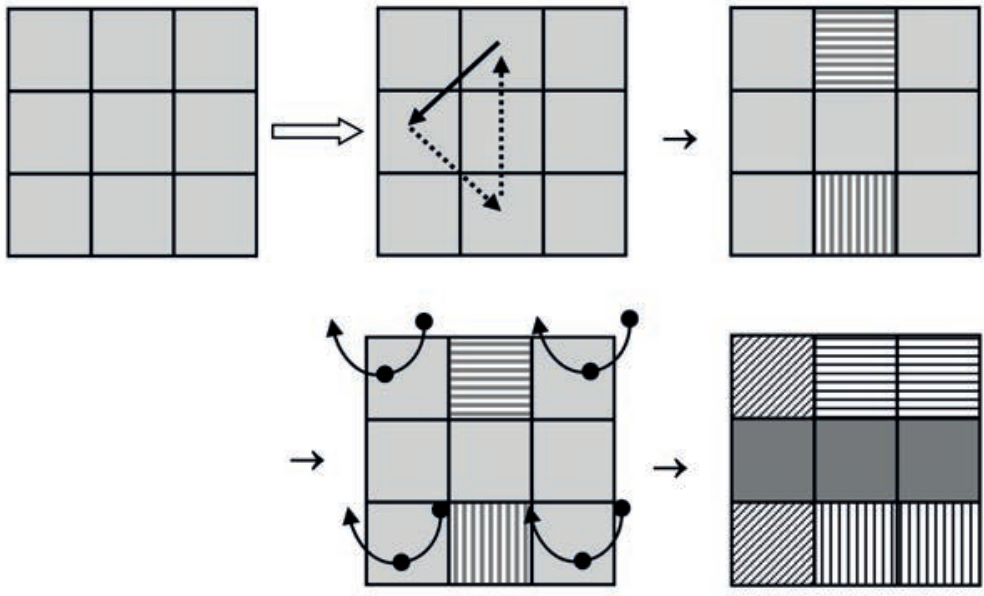

$$
\mathrm{I}_{1} \mathrm{~S}
$$

\subsubsection{Acción de $\hat{\mathbf{c}}\left(\mathbf{I}_{1} \mathbf{S}\right)$ sobre sus aristas iniciales $\hat{\mathbf{c}}\left(\mathbf{I}_{1} \mathbf{S}\right)$}

realiza una permutación en sentido antihorario de tres de las aristas distintas de la derecha (permanece invariante), provocando permutaciones en los stickers de algunas de estas.

De modo preciso:

- La arista superior pasa a ser la arista izquierda;

- La arista izquierda pasa a ser la inferior y sus stickers permutan;

- La arista inferior pasa a ser la superior y sus stickers permutan. 


\subsubsection{Acción de $\hat{\mathbf{c}}\left(\mathrm{I}_{1}{ }^{\mathrm{S}}\right)$ sobre sus vértices iniciales}

- Los vértices frontales (inferior izquierdo e inferior derecho) reciben una torsión "antihoraria" de tres giros (1.6.3.3).

- Los vértices de atrás (superior izquierdo y superior derecho) reciben una torsión "horaria" de tres giros (1.6.3.3).

\subsection{La Cruz en su forma canónica}

Este OLL hace parte de un grupo denominados "Los siete de cruz ampliada". El nombre obedece a que todos tienen las aristas bien orientadas, formando con el centro de la capa superior una cruz.

La "Cruz" tiene simetría con respecto a sus lados izquierdo y derecho. Es además muy sencillo de memorizar en su forma canónica pues su constructor es igual a su deconstructor, resultando una ampliación del $\mathrm{I}_{1}$ en su forma canónica.

\subsubsection{Motivación $\mathrm{Cruz}_{1}=\hat{\mathrm{c}}\left(\mathrm{I}_{1}\right) \mathrm{d}\left(\mathrm{T}_{1}\right)$}

$\mathbf{C r u z}_{1}=\hat{\mathbf{c}}\left(\mathbf{I}_{\mathbf{1}}\right) \mathbf{d}\left(\mathbf{T}_{\mathbf{1}}\right)$. Apliquemos el deconstructor canónico de $\mathrm{T}_{1}$ (según lo expuesto en 1.7.3) a la $\mathrm{I}_{1}$ de más abajo $\left(=\hat{\mathrm{c}}\left(\mathrm{I}_{1}\right)\right)$

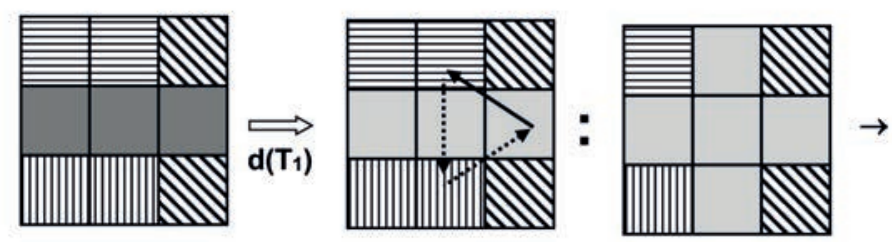

$\mathbf{I}_{1}$
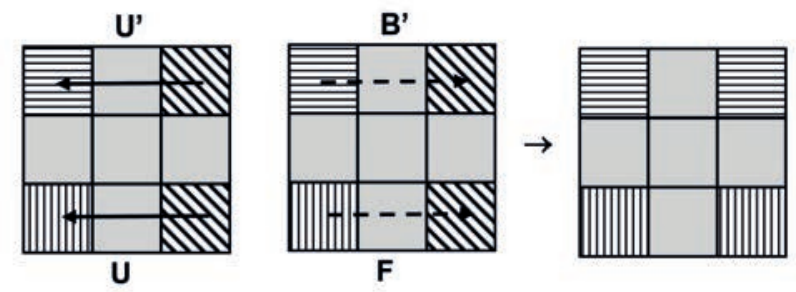

Cruz $_{1}$ 
Así $c\left(\right.$ Cruz $\left._{1}\right)=\widehat{\mathbf{C}}\left(\mathrm{I}_{1}\right) \mathrm{d}\left(\mathrm{T}_{1}\right)=\left[\mathrm{F}\left(\mathrm{RUR}^{\prime} \mathrm{U}^{\prime}\right) 2 \mathrm{~F}^{\prime}\right]\left(\mathrm{FRUR} \mathrm{R}^{\prime} \mathrm{U}^{\prime} \mathrm{F}^{\prime}\right)=\mathbf{F}\left(\mathrm{RUR}^{\prime} \mathrm{U}^{\prime}\right) \mathbf{3}$ F'

Pero también, como lo puede comprobar el lector usando su cubo de Rubik, es válido que $\mathrm{Cruz}_{1}$ se deconstruye con $\mathrm{c}\left(\mathrm{Cruz}_{1}\right)$.

4.6.2 Constructor/deconstructor canónicos de Cruz

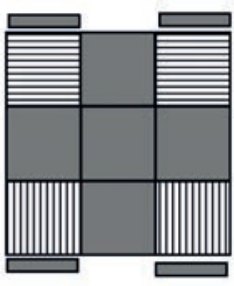

$\hat{\mathbf{c}}\left(\mathrm{Cruz}_{1}\right)=\hat{\mathrm{d}}\left(\mathrm{Cruz}_{1}\right)=\mathrm{F}\left(\mathrm{RUR}^{\prime} \mathrm{U}^{\prime}\right) 3 \mathrm{~F}^{\prime}$

4.6.3 Dinámica del $\widehat{\mathrm{C}}\left(\mathrm{Cruz}_{1}\right)$ en la capa superior
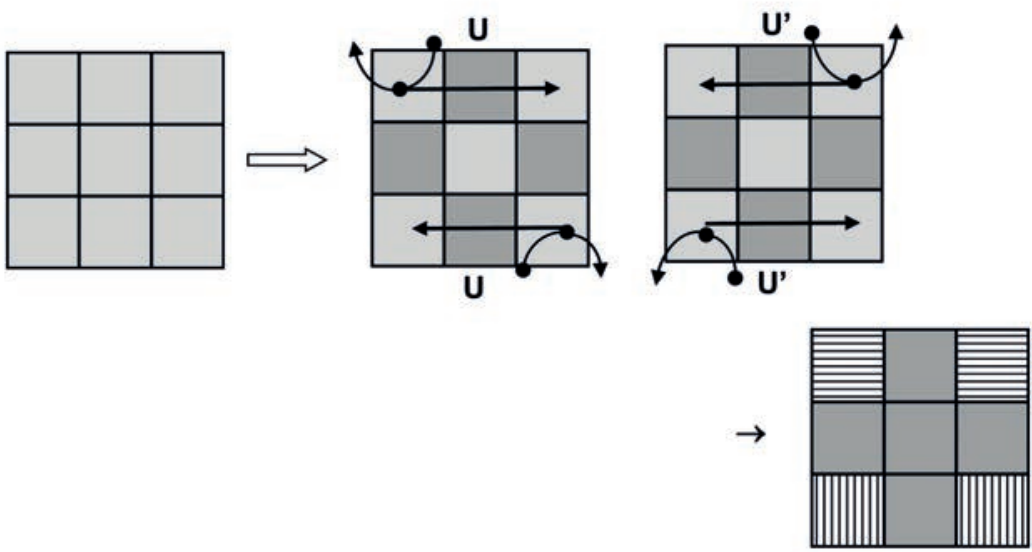

Cruz1

\subsubsection{Acción de $\widehat{\mathbf{C}}\left(\mathrm{Cruz}_{1}\right)$ sobre sus aristas iniciales}

$\widehat{\mathbf{c}}\left(\mathrm{Cruz}_{1}\right)$ deja invariantes las aristas: bien acomodadas (bien posicionadas y bien orientadas)

\subsubsection{Acción $\widehat{\mathbf{c}}\left(\mathrm{Cruz}_{1}\right)$ sobre sus vértices iniciales}

- Los vértices opuestos inferior derecho y superior izquierdo reciben una torsión "horaria" de tres giros (1.6.3.3) y luego un movimiento $\mathrm{U}$. 
- Los vértices opuestos inferior izquierdo y superior derecho reciben una torsión "antihoraria" de tres giros (1.6.3.3) y luego un movimiento U'.

\subsection{Nuevos constructores/deconstructores de la Cruz ${ }_{1}$ El PLL V}

Esta nueva forma solo usa los movimientos $\mathrm{R}$ y $\mathrm{U}$, y será usada en el próximo capítulo; razón por la cual es bueno que el lector la practique y la memorice. Al interactuar esta nueva forma con la canónica de la surge la permutación PLL V, de la cual también tratamos en esta sección.

\subsubsection{Dos formas de construir/deconstruir Cruz}
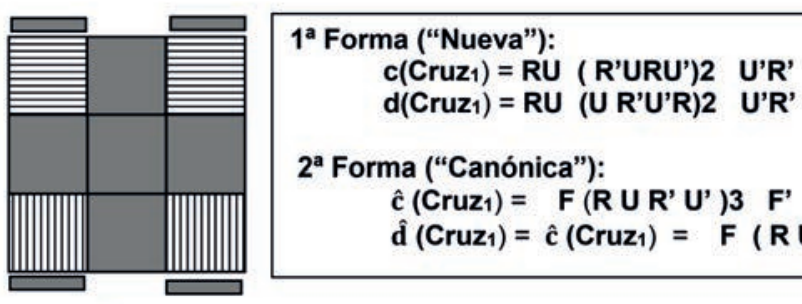

Cruz1

\subsubsection{La no congruencia de las dos formas}

En la configuración de la $2^{\text {a }}$ forma todas las aristas están bien acomodadas, lo que no sucede con la $1^{\text {a }}$ forma.

\subsubsection{La semejanza de la configuración de las dos formas}

Intercambiando constructor/deconstructor. La permutación "V".

Sean $\mathbf{m}=\mathbf{c}\left(\mathbf{C r u z}_{\mathbf{1}}\right)$ : RU( R'URU')2 U'R' y $\mathbf{n}=\widehat{\mathbf{c}}\left(\mathbf{C r u z}_{\mathbf{1}}\right)$ : F ( R U R' U')3 F'.

Al ejecutar los constructores de ambas formas se obtiene la misma configuración por semejanza:

$$
\widetilde{\mathrm{C}_{\mathrm{m}}}=\widetilde{\mathbf{C}_{\mathrm{n}}}
$$




\subsubsection{Situación 1.p: c( $\left.\mathrm{Cruz}_{1}\right) \hat{\mathrm{d}}\left(\mathrm{Cruz}_{1}\right)$}

donde

$$
\begin{aligned}
& \mathrm{c}\left(\mathrm{Cruz}_{1}\right)=\mathrm{RU}\left(\mathrm{R}^{\prime} \mathrm{URU}^{\prime}\right) 2 \mathrm{U}^{\prime} \mathrm{R}^{\prime} \\
& \hat{\mathrm{d}}\left(\mathrm{Cruz}_{1}\right)=\mathrm{F} \text { (R U R' U')3 F' }
\end{aligned}
$$

Diseñar la configuración $I_{1}$ a partir de la $1^{\text {a }}$ forma (constructor $\mathrm{c}\left(\mathrm{Cruz}_{1}\right)$ ) y orientar el cubo usando la $2^{\mathrm{a}}$ forma (deconstructor $\left.\mathrm{d}\left(\mathrm{Cruz}_{1}\right)\right)$

Sea $C_{p}$ la configuración resultante de ejecutar $\mathbf{p}$ desde el cubo en su estado original. Sucede que $\mathrm{C}_{\mathrm{p}}=\mathrm{C}_{\text {т.o. }}$ (cubo totalmente orientado, pero no totalmente organizado).

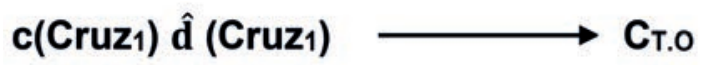

Para llevar el cubo a su estado original aplicar el PLL: U2 V U

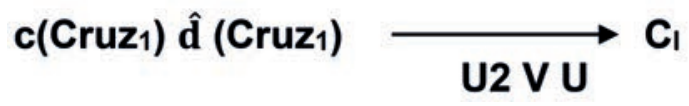
donde $\mathbf{V}=\mathbf{U} 2 \mathbf{U b} \mathbf{E} \mathbf{U}^{\prime}$

Recordar: $E=\left(R^{\prime}\right.$ UL' D2 LU' R) (L'UR' D2 RU' L), 2.9.2

\subsubsection{Situación 2. q: $\widehat{c}\left(\mathrm{Cruz}_{1}\right) \mathrm{d}\left(\mathrm{Cruz}_{1}\right)$,} donde:

$$
\begin{aligned}
& \hat{c}\left(\mathrm{Cruz}_{1}\right)=\mathrm{F}\left(\mathrm{R} \mathrm{U} \mathrm{R}^{\prime} \mathrm{U}^{\prime}\right) 3 \mathrm{~F}^{\prime} \\
& \mathrm{d}\left(\mathrm{Cruz}_{1}\right)=\mathrm{RU}\left(\mathrm{U}^{\prime} \mathrm{R}^{\prime} \mathrm{R}\right) 2 \text { U'R' }
\end{aligned}
$$

Diseñar la configuración Cruz ${ }_{1}$ a partir de la $2^{\text {a }}$ forma (constructor $\widehat{\mathbf{C}}\left(\mathrm{Cruz}_{1}\right)$ ) y orientar el cubo usando la $1^{\mathrm{a}}$ forma deconstructor $\mathrm{d}\left(\mathrm{Cruz}_{1}\right)$

Sea $\mathrm{C}_{\mathrm{q}}$ la configuración resultante de ejecutar $\mathbf{q}$ desde el cubo en su estado original. Sucede que $\mathrm{C}_{\mathrm{q}}=\mathrm{C}_{\text {T.o. }}$ (cubo totalmente orientado, pero no totalmente organizado). 


$$
\text { c(Cruz } 1) \text { d̂ (Cruz } 1)
$$

$\mathbf{C}_{\mathrm{T} . \mathrm{O}}$

Para llevar el cubo a su estado original aplicar el PLL: U' V U2.

$$
\mathrm{c}\left(\mathrm{Cruz}_{1}\right) \hat{\mathrm{d}} \text { (Cruz1) } \underset{\text { U' V U2 }}{\longrightarrow} \mathrm{C}_{1}
$$

$$
\operatorname{con} \mathbf{V}=\mathbf{U} 2 \mathbf{U b} \mathbf{E} \mathbf{U}^{\prime}
$$

4.8 La Permutación "V": Su canónica y configuración

4.8.1 Motivación: U2 Ub E U’ =V
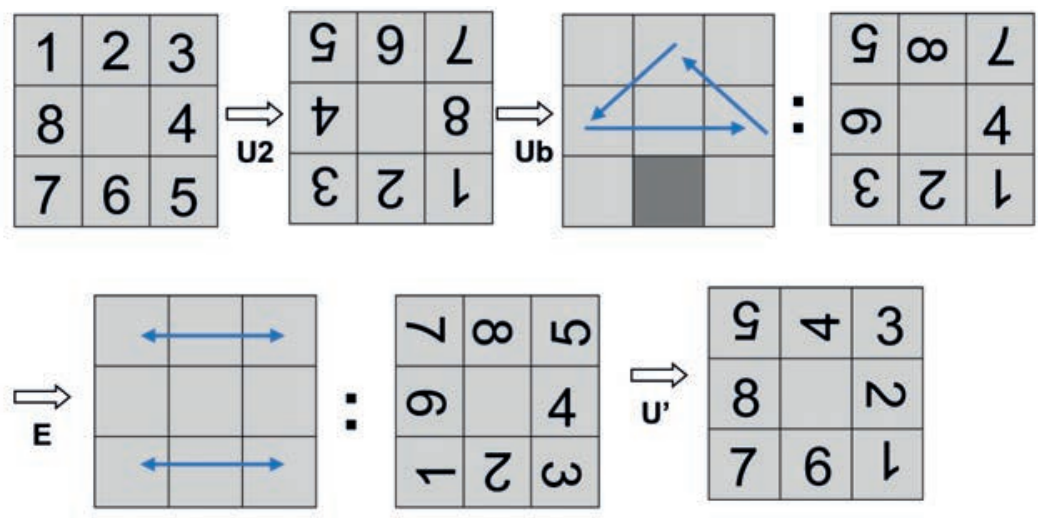

En síntesis:
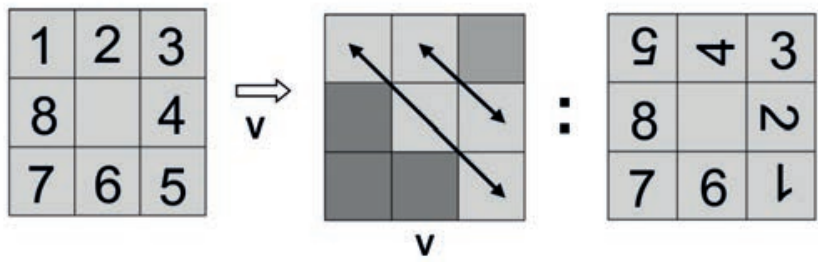

4.8.2 Forma canónica del PLL "V"

(Permutación de los vértices diagonales que

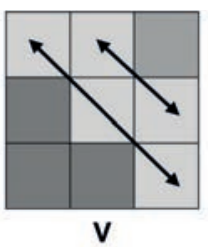
están en la diagonal NO principal y las aristas diagonales que están arriba de esa diagonal: las aristas superior y derecha).

$$
\text { (R' U2 R U2) (L U’ R' U L' U L U’ R U L') }
$$


Con el cubo en su estado original y ejecutando "V" surge esa configuración. Una vez hecho "V" para volver al cubo en su estado original debe repetir tal ejecución.

\subsubsection{Configuración para aplicar el algoritmo "V"}

La configuración "V" es muy parecida a las "A" en el sentido de hay dos y solamente dos bloques de dos sticker juntos cada uno del mismo color en dos lados superiores contiguos, formando una " $V$ " de dos colores. Pero la diferencia radica que mientras en "A" los stickers restantes en ambos bloques son de igual color, en la configuración "V" son de color distintos ${ }^{21}$.

Para aplicar correctamente el algoritmo "V" el vértice común en los antes mencionados bloques debe ubicarse como vértice inferior izquierdo $\left(\mathbf{V}_{\text {I.I. }}\right)$ de la capa superior.

\section{Síntesis tabular OLL “Canónica” (Capítulo 4) 3 OLL para practicar y memorizar}

\begin{tabular}{|c|c|c|}
\hline 4.1.2 $\mathrm{I}_{1}$ & $\begin{array}{l}\hat{\mathbf{c}}\left(\mathbf{I}_{1}\right)=\mathbf{F}\left(\mathbf{R} \mathbf{U} \mathbf{R}^{\prime} \mathbf{U}^{\prime}\right) \mathbf{2} \mathbf{F}^{\prime} \\
\hat{\mathbf{d}}\left(\mathbf{I}_{1}\right)=\mathbf{F}\left(\mathbf{U} \mathbf{R} \mathbf{U}^{\prime} \mathbf{R}^{\prime}\right) 2 \mathbf{F}^{\prime}\end{array}$ & E" \\
\hline 4.5.2 $\mathbf{I}_{1}{ }^{\mathrm{s}}$ & $\begin{array}{l}\hat{\mathbf{c}}\left(\mathbf{I}_{1}{ }^{S}\right)=\mathbf{F}^{\prime}\left(\mathbf{L}^{\prime} \mathbf{U}^{\prime} \mathbf{L} \mathbf{U}\right) 2 \mathbf{F} \\
\hat{\mathbf{d}}\left(\mathbf{I}_{1}{ }^{S}\right)=\mathbf{F}^{\prime}\left(\mathbf{U}^{\prime} \mathbf{L}^{\prime} \mathbf{U} \mathbf{L}\right) 2 \mathbf{F}\end{array}$ & 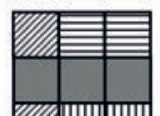 \\
\hline 4.6.2 $\mathrm{Cruz}_{1}$ & $\begin{array}{c}\hat{\mathbf{c}}\left(\mathrm{Cruz}_{1}\right)=\mathbf{F}\left(\mathbf{R} \mathbf{U} \mathbf{R}^{\prime} \mathbf{U}^{\prime}\right) \mathbf{3} \mathbf{F}^{\prime} \\
\hat{\mathbf{d}}\left(\mathrm{Cruz}_{1}\right)=\hat{\mathbf{c}}\left(\mathrm{Cruz}_{1}\right)\end{array}$ & 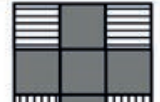 \\
\hline
\end{tabular}

21 De modo preciso: cada uno de los stickers restante de esos lados es de color opuesto al del bloque de dos stickers juntos (= color) donde se hallan tales stickers. 
Síntesis tabular PLL "Canónica” (Capítulo 4)

Para practicar y memorizar: 5 PLL

\begin{tabular}{|r|r|l|l|}
\hline 4.3 .1 & & \\
\hline
\end{tabular}




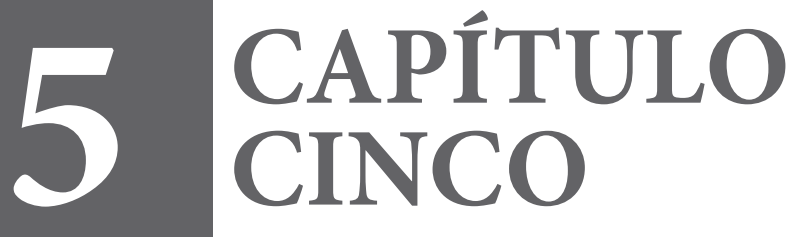




\section{La primera Mini J y Las $4 \mathrm{G}$}

La primera Mini J, notada Mini $J_{1}$ o Mini J $(2,1,1,2)$, es la inversa de la $\mathrm{I}_{1}$ tratada en el capítulo anterior; Innegablemente es uno de los algoritmos, como veremos en próximos capítulos, más usados para construir/deconstruir configuraciones en las cuales se pueda distinguir una Mini J y que bien podríamos llamar las "Mini J ampliadas".

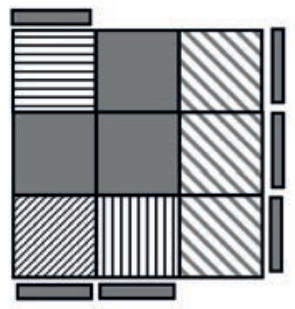

$\mathrm{mJ}_{3}$

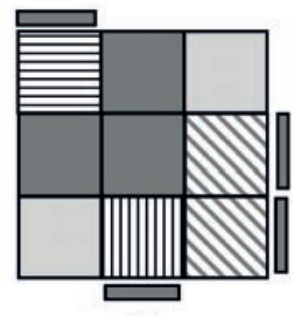

W

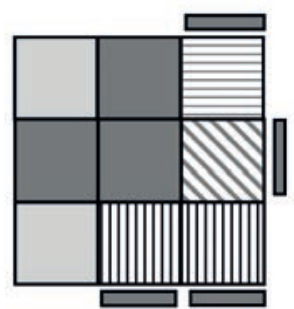

$\mathbf{P}_{2}$

\subsection{La Primera Mini J: $\mathrm{mJ}_{1}$ y su forma canónica}

Mini $J_{1}$ es la inversa de la canónica de $I_{1}$ dada en la sección 4.1.2 (segunda forma). Mini $J_{1}=$ Mini $J(2,1,1,2)$.

\subsubsection{Motivación:}

$\mathrm{T}_{1}+\mathrm{T}_{1}=\mathrm{mJ}_{1}$. Apliquemos el constructor canónico de $\mathrm{T}_{1}$ (según lo expuesto en 1.6.3) a la $\mathrm{T}_{1}$ de más abajo $\left(=\mathrm{c}\left(\mathrm{T}_{1}\right)\right)$
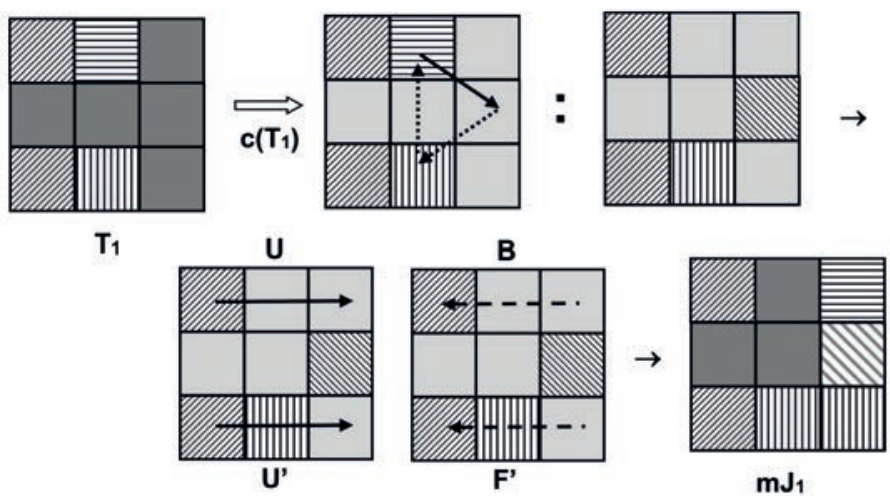
5.1.2 Constructor/deconstructor canónicos de “ $\mathrm{mJ}_{1}$ ” (= $\left.\mathrm{I}_{1}{ }^{\prime}\right)$

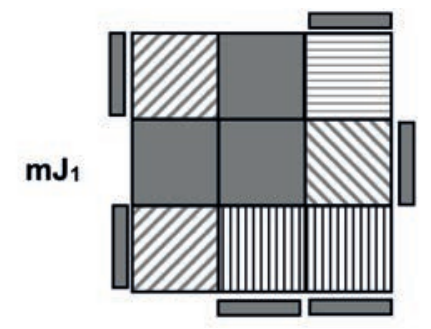

$$
\begin{aligned}
& \hat{\mathbf{c}}\left(m J_{1}\right)=F\left(U R U^{\prime} R^{\prime}\right) 2 F^{\prime} \\
& \hat{\mathbf{d}}\left(m J_{1}\right)=F\left(R U R^{\prime} U^{\prime}\right) 2 F^{\prime}
\end{aligned}
$$

5.1.3 Dinámica del $\widehat{\mathrm{C}}\left(\mathrm{mJ}_{1}\right)$ en la capa superior
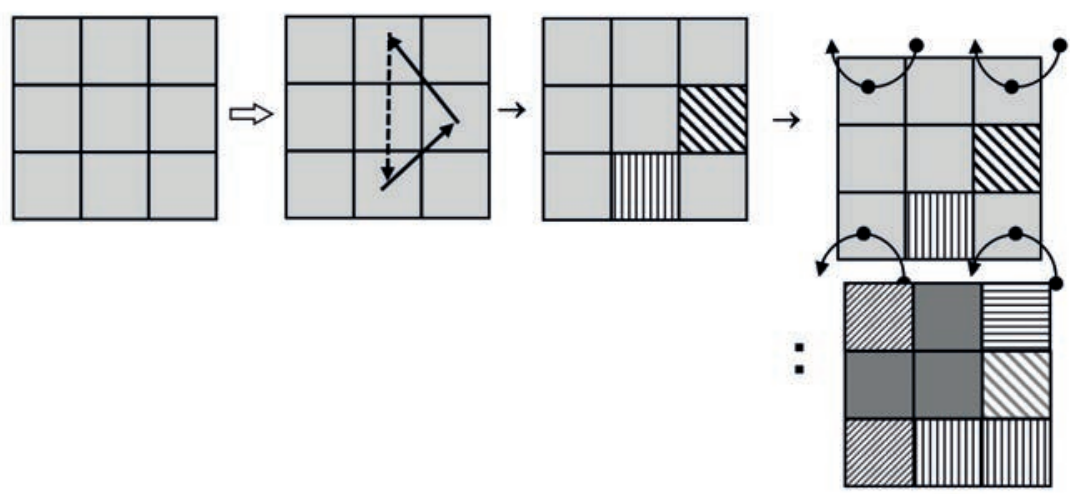

$\mathrm{mJ}_{1}$

5.1.3.1 Acción de $\widehat{\mathrm{c}}\left(\mathrm{mJ}_{1}\right)$ sobre sus aristas iniciales: $\widehat{\mathrm{c}}$ $\left(\mathbf{m J}_{1}\right)$ realiza una permutación en sentido antihorario de tres de las aristas distintas de la izquierda (permanece invariante), provocando permutaciones en los stickers de algunas de estas.

De modo preciso:

- La arista derecha pasa a ser la arista superior.

- La arista superior pasa a ser la inferior y sus stickers permutan.

- La arista inferior pasa a ser la derecha y sus stickers permutan. 


\subsubsection{Acción de $\widehat{\mathrm{c}}\left(\mathrm{mJ}_{1}\right)$ sobre sus vértices iniciales}

- Los vértices frontales (inferior izquierdo e inferior derecho) reciben una torsión "antihoraria" de tres giros (1.6.3.3).

- Los vértices de atrás (superior izquierdo y superior derecho) reciben una torsión "horaria" de tres giros (1.6.3.3).

5.2 Un nuevo constructor de $\mathrm{mJ}_{1}$. Inicio de los PLL “Ga y Gb”

5.2.1 Motivación:

$\mathrm{I}_{1}^{\mathrm{S}} \mathrm{T}_{2}=\mathrm{mJ}_{1}$. Apliquemos el constructor canónico de $\mathrm{T}_{2}$ (según lo expuesto en 2.1.3) a la $\mathrm{I}_{1}^{\mathrm{S}}$.
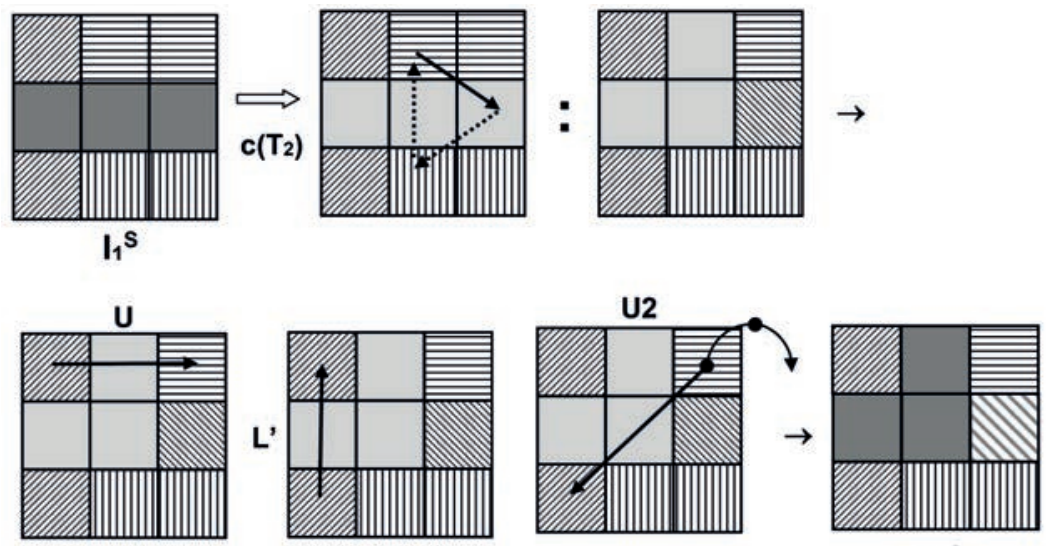

$\mathrm{mJ}_{1}$

5.2.2 Nuevo constructor/deconstructor de $\mathrm{mJ}_{1} \mathrm{o} \mathrm{mJ}(2,1,1,2)$
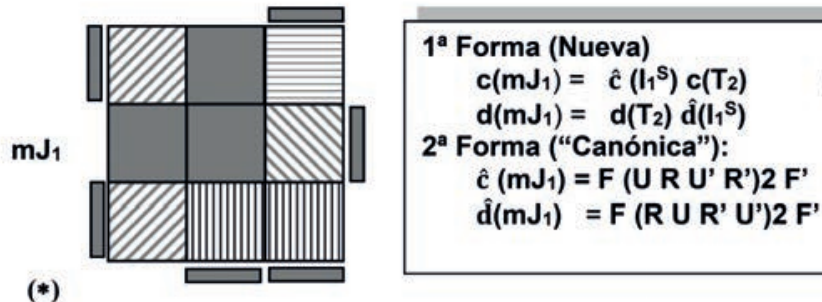

$2^{\text {a }}$ Forma ("Canónica"):

$\hat{c}\left(m J_{1}\right)=F\left(U R U^{\prime} R^{\prime}\right) 2 F^{\prime}$

$\hat{d}\left(m^{\prime} J_{1}\right)=F\left(R \cup R^{\prime} U^{\prime}\right) 2 F^{\prime}$

$\hat{\mathbf{c}}\left(\mathbf{I}_{1}{ }^{\mathrm{S}}\right) \mathbf{c}\left(\mathbf{T}_{2}\right)=\mathrm{F}^{\prime}$ ( L' U' L U)2 F (F R' F' R U R U' R')

$d\left(T_{2}\right) \hat{d}\left(I_{1}{ }^{S}\right)=(R$ U R' U' R' F R F') F' (U' L' U L)2 F 


\subsubsection{La no congruencia de las dos formas}

Una diferencia radica en los vértices de la cara superior: Con la $2^{\text {a }}$ forma todos los vértices quedan bien posicionados, cosa que no se da con la $1^{\text {a }}$ forma.

\subsubsection{La semejanza en la configuración de las dos formas}

$\mathrm{Al}$ ejecutar los constructores de ambas formas se obtiene la misma configuración por semejanza:

$$
\widetilde{\mathrm{C}_{\mathrm{p}}}=\widetilde{\mathrm{C}_{\mathrm{q}}}
$$

siendo $\mathrm{p}=\mathrm{c}\left(\mathrm{mJ}_{1}\right)=\hat{\mathbf{c}}\left(\mathbf{I}_{1}{ }^{\mathrm{S}}\right) \mathbf{c}\left(\mathbf{T}_{2}\right) \quad \mathbf{y} \quad \mathrm{q}=\hat{\mathbf{c}}\left(\mathbf{m J _ { 1 }}\right)=\mathbf{F}\left(\mathbf{U} \mathbf{R} \mathbf{U}^{\prime} \mathbf{R}^{\prime}\right) \mathbf{2} \mathbf{F}^{\prime}$

A continuación, se verificará lo anterior también de modo algebraico Intercambiando constructor/deconstructor.

\subsubsection{Situación 1. p: $\mathrm{c}\left(\mathrm{mJ}_{1}\right) \hat{\mathbf{d}}\left(\mathrm{mJ}_{1}\right)$} donde

$$
\mathbf{c}\left(\mathbf{m} \mathbf{J}_{1}\right)=\hat{\mathbf{c}}\left(\mathbf{I}_{1}{ }^{\mathbf{S}}\right) \mathbf{c}\left(\mathbf{T}_{2}\right) \text { y } \hat{\mathbf{d}}\left(\mathbf{m} \mathbf{J}_{1}\right)=\mathbf{F}\left(\mathbf{R} \mathbf{U} \mathbf{R}^{\prime} \mathbf{U}^{\prime}\right) \mathbf{2} \mathbf{F} \text { ' }
$$

Sea $C_{p}$ la configuración resultante de ejecutar $\mathbf{p}$ desde el cubo en su estado original. Sucede que $\mathrm{C}_{\mathrm{p}}=\mathrm{C}_{\text {т.o. }}$ (cubo totalmente orientado, pero no totalmente organizado).

$$
\mathbf{c}\left(\mathbf{m J}_{1}\right) \hat{\mathbf{d}}\left(\mathbf{m J}_{\mathbf{1}}\right) \longrightarrow \mathbf{C}_{\mathrm{T} .0}
$$

Para llevar el cubo a su estado original puede aplicarse el PLL: UGaU'

$$
c\left(m J_{1}\right) \hat{d}\left(m J_{1}\right) \underset{U G a U}{\longrightarrow} C_{1}
$$

donde $\mathbf{G a}=\mathbf{U}^{\prime} \mathbf{A a}^{\mathrm{s}} \mathbf{U a} \mathbf{U}$

\subsubsection{Situación 2. q: $\widehat{c}\left(\mathrm{~mJ}_{1}\right) \mathrm{d}\left(\mathrm{mJ}_{1}\right)$} donde

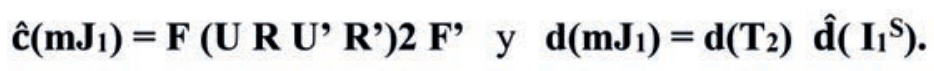

El lector puede comprobar la validez del esquema siguiente:

$$
\hat{c}\left(m J_{1}\right) d\left(m J_{1}\right) \underset{U G b U}{\longrightarrow} C_{1}
$$

donde $\mathbf{G b}=\mathbf{G a}^{\prime}=\mathbf{U}^{\prime} \mathbf{U b}$ AbS U 
5.3 La permutación "Ga”

5.3.1 Motivación: U’ $\mathrm{Aa}^{\mathrm{s}} \mathrm{Ua} \mathrm{U}=$ "Ga”

\begin{tabular}{|l|l|l|}
\hline 1 & 2 & 3 \\
\hline 8 & & 4 \\
\hline 7 & 6 & 5 \\
\hline
\end{tabular}
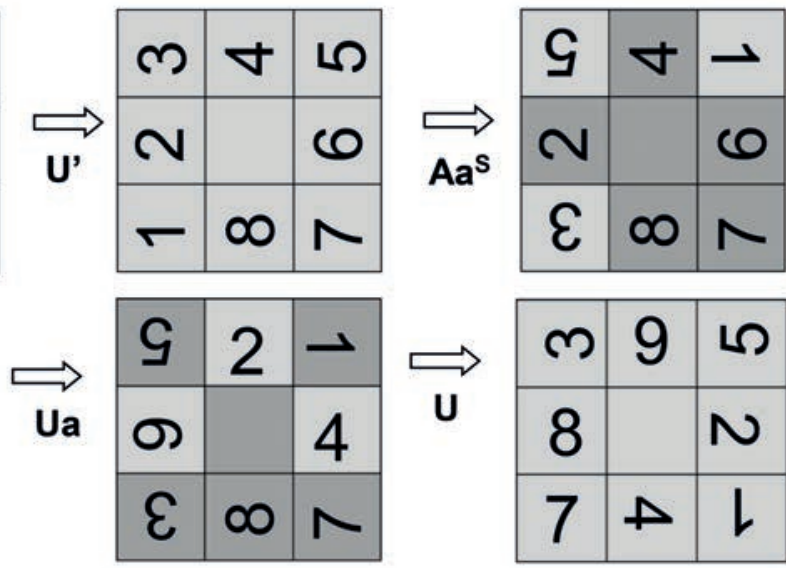

En síntesis:
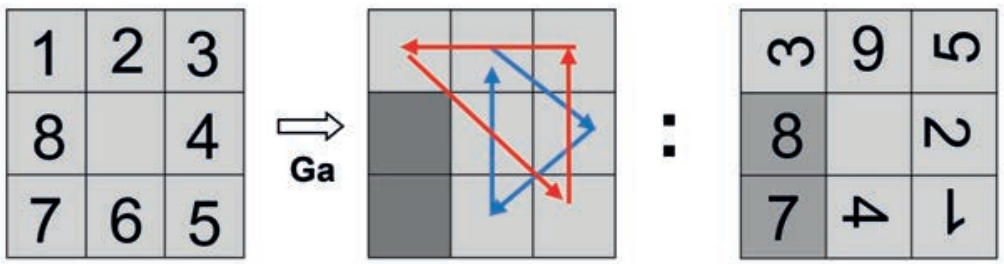

5.3.2 Objetivo de Ga (en la capa superior)

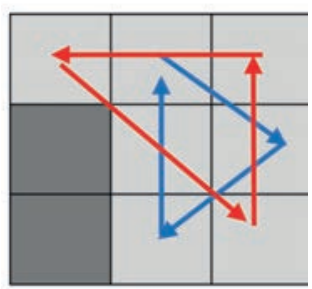

Permutar en sentido horario las tres aristas distintas a la izquierda $y$, permutar en sentido antihorario los tres vértices distintos al inferior izquierdo.

Ga 


\subsubsection{Forma canónica del PLL “Ga”}

\section{L' U'L F2 D R' U R U'R D' F2}

Con el cubo en su estado original y ejecutando:

$$
\mathrm{Gb}=\mathrm{Ga}^{-1}=\mathrm{F} 2 \text { D R' U R' U' R D' F2 L' U L surge la }
$$
configuración dada en la figura de arriba a la derecha.

Al aplicar a esta configuración el algoritmo Ga se orienta todo el cubo (en este caso en especial se vuelve al cubo en su estado original).

\subsubsection{Configuración y Ayuda aplicativa de la permutación "Ga"}

- Nos concentramos únicamente en la capa superior, especialmente en los lados de arriba de las caras laterales.

- Hay una y sólo una cara lateral donde en su lado de arriba se observa un "bloque de dos sticker contiguos "a derecha" del mismo color" (opuesto al del sticker lateral del vértice que se halla en dicho lado). Ese lado de arriba se ubica en la cara izquierda del cubo (no importa cómo quede el resto del cubo).

- Al colocar en la cara izquierda el lado antes descrito, se observa en la cara frontal que los tres stickers del lado superior son de colores distintos, pero los dos primeros stickers de izquierda a derecha $(\rightarrow)$ tienen colores opuestos.

- De tener una configuración en la capa superior como la descrita en los dos párrafos anteriores se aplica la permutación $\mathrm{Ga}$. Al final con movimientos U se organiza totalmente el cubo. 
5.4 La permutación “Gb”: Inversa de Ga

5.4.1 Motivación: U’ Ub AbS U = “Gb”
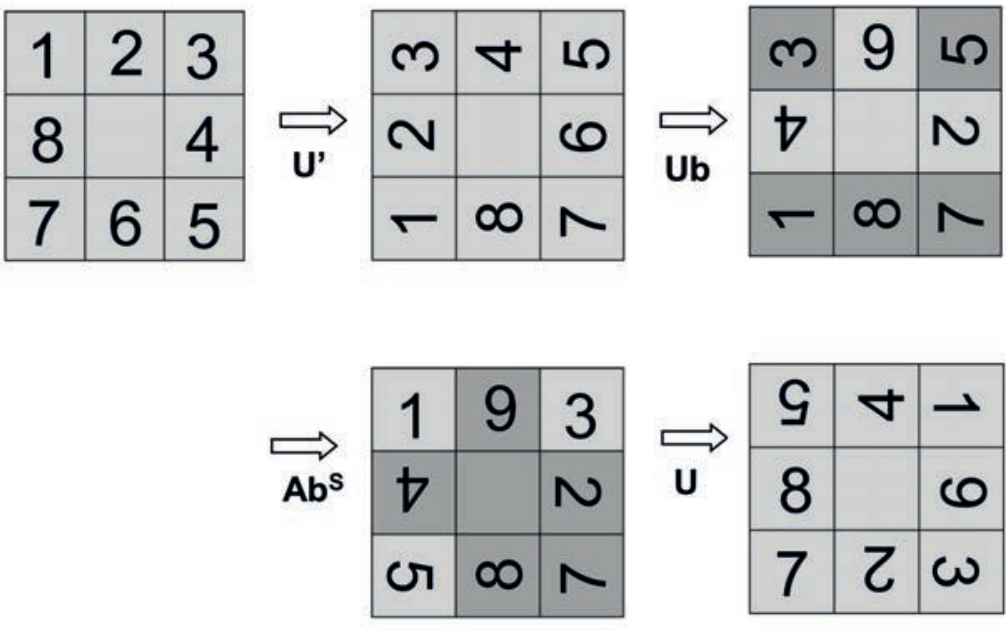

En síntesis:
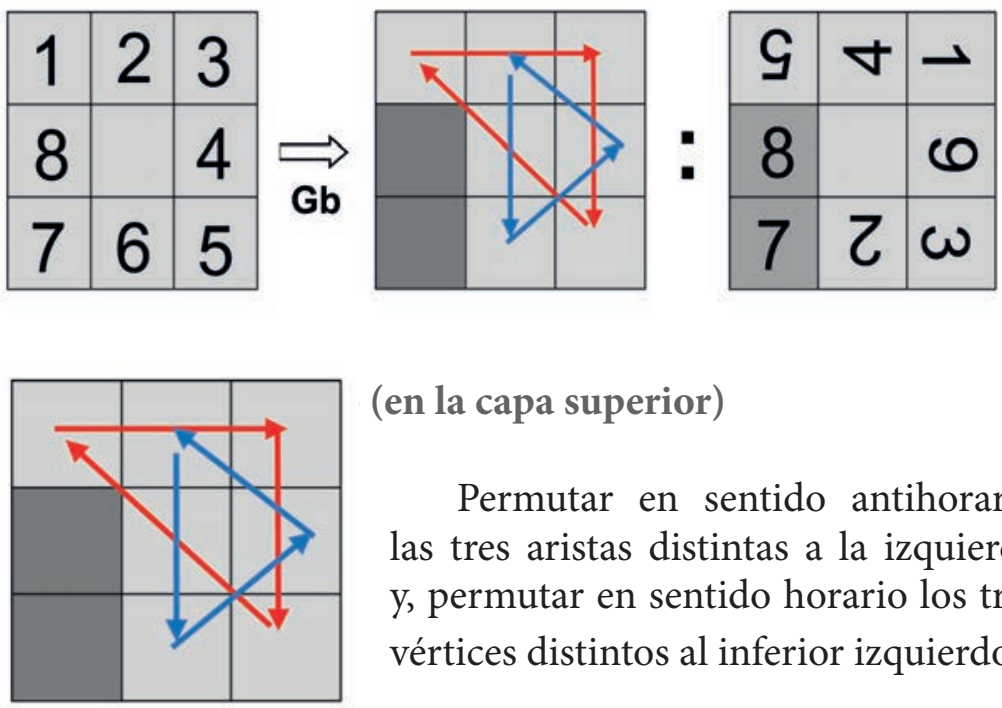

(en la capa superior)

Permutar en sentido antihorario las tres aristas distintas a la izquierda $y$, permutar en sentido horario los tres vértices distintos al inferior izquierdo.

\section{Gb}




\subsubsection{Forma canónica del PLL “Gb"}

\section{F2 D R' U R' U’ R D' F2 L' U L}

Con el cubo en su estado original y ejecutando:

$\mathrm{Ga}=\mathrm{Gb}^{-1}=\mathrm{L}^{\prime} \mathrm{U}^{\prime} \mathrm{L} \quad$ F2 D R' U R $\quad$ U' R D' F2 surge la configuración dada en la figura de arriba a la derecha.

Al aplicar a esta configuración el algoritmo Gb se orienta todo el cubo (en este caso en especial se vuelve al cubo en su estado original).

\subsubsection{Configuración y Ayuda aplicativa de la permutación “Gb”}

- Nos concentramos únicamente en la capa superior, especialmente en los lados de arriba de las caras laterales.

- Hay una y sólo una cara lateral donde en su lado de arriba se observa un "bloque de dos sticker contiguos "a derecha" del mismo color."

Ese lado de arriba se ubica en la cara izquierda del cubo (no importa cómo quede el resto del cubo).

- Al colocar en la cara izquierda el lado antes descrito, se observa en la cara frontal que "los tres stickers del lado superior son de colores distintos" y los de los primeros de izquierda a derecha $(\rightarrow)$ "no son opuestos."

- De tener una configuración en la capa superior como la descrita en los dos párrafos anteriores se aplica la permutación $\mathrm{Gb}$. Al final con movimientos U se organiza totalmente el cubo.

Una vez tratadas dos de las $4 \mathrm{G}$ aprovechamos para estudiar las otras dos (simétricas de las ya descritas) en aras de completar su buen conocimiento. Precisamente lo hacemos en las siguientes dos secciones. 
5.5 La permutación "Gd”: Simétrica de "Ga”

5.5.1 Motivación: U Aa Ub U” = “Gd”
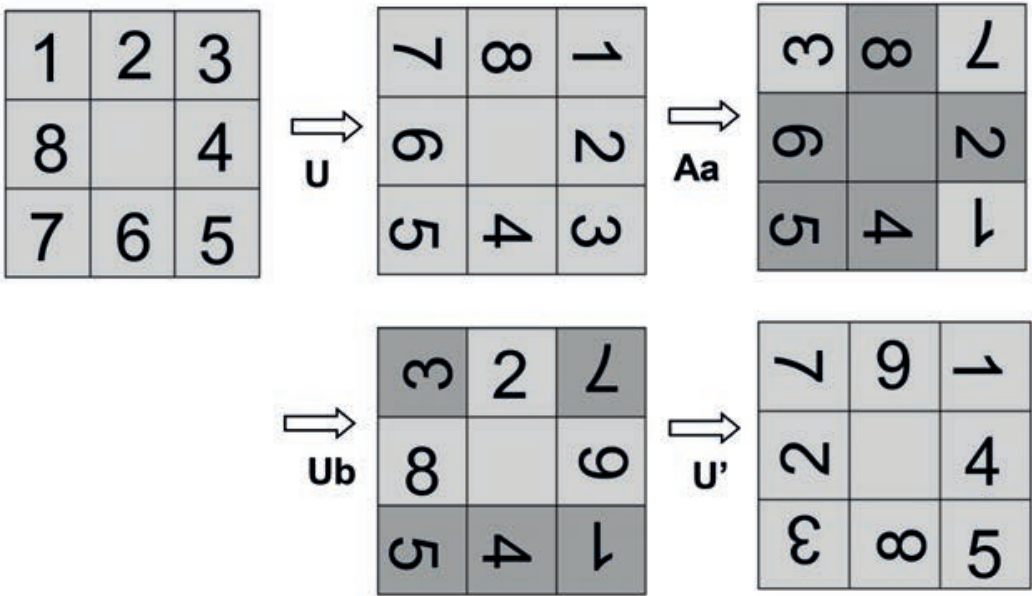

En síntesis:
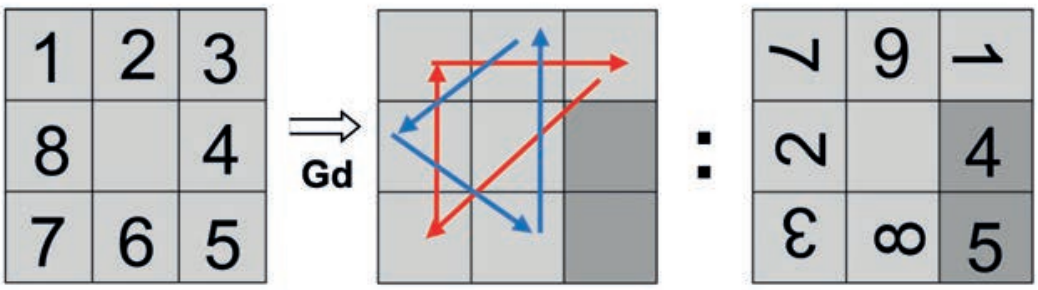

\subsubsection{Objetivo de Gd (en la capa superior)}

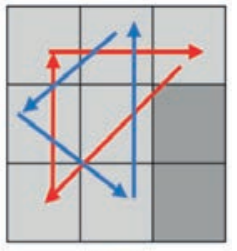

Gd

Permutar en sentido antihorario las tres aristas distintas a la derecha $y$, permutar en sentido horario los tres vértices distintos al inferior derecho.

5.5.3 Forma canónica del PLL “Gd”

$$
\text { RUR' F2 D' L U' L' U L'D F2 }
$$

Con el cubo en su estado original y ejecutando: 
$\mathrm{Gc}=\mathrm{Gd}^{-1}=\mathrm{F} 2 \mathrm{D}^{\prime} \mathrm{L} \mathrm{U}^{\prime} \mathrm{L}$ U L' D F2 R U' R' surge la configuración dada en la figura de arriba a la derecha. Al aplicar a esta configuración el algoritmo Gd se orienta todo el cubo (en este caso en especial se vuelve al cubo en su estado original).

\subsubsection{Configuración y Ayuda aplicativa de la permutación "Gd"}

- Nos concentramos únicamente en la capa superior, especialmente en los lados de arriba de las caras laterales.

- Hay una y sólo una cara lateral donde en su lado de arriba se observa un "bloque de dos sticker contiguos "a izquierda" del mismo color" (opuesto al del sticker lateral del vértice que se halla en dicho lado). Ese lado de arriba se ubica en la cara derecha del cubo (no importa cómo quede el resto del cubo).

- Al colocar en la cara derecha el lado antes descrito, se observa en la cara frontal que los tres stickers del lado superior son de colores distintos, pero los dos primeros stickers de derecha a izquierda $(\leftarrow)$ tienen colores opuestos.

- De tener una configuración en la capa superior como la descrita en los dos párrafos anteriores se aplica la permutación Gd Al final con movimientos U se organiza totalmente el cubo. 
5.6 La permutación “Gc”: Simétrica de Gb

5.6.1 Motivación: U Ua Ab U’ = “Gc”

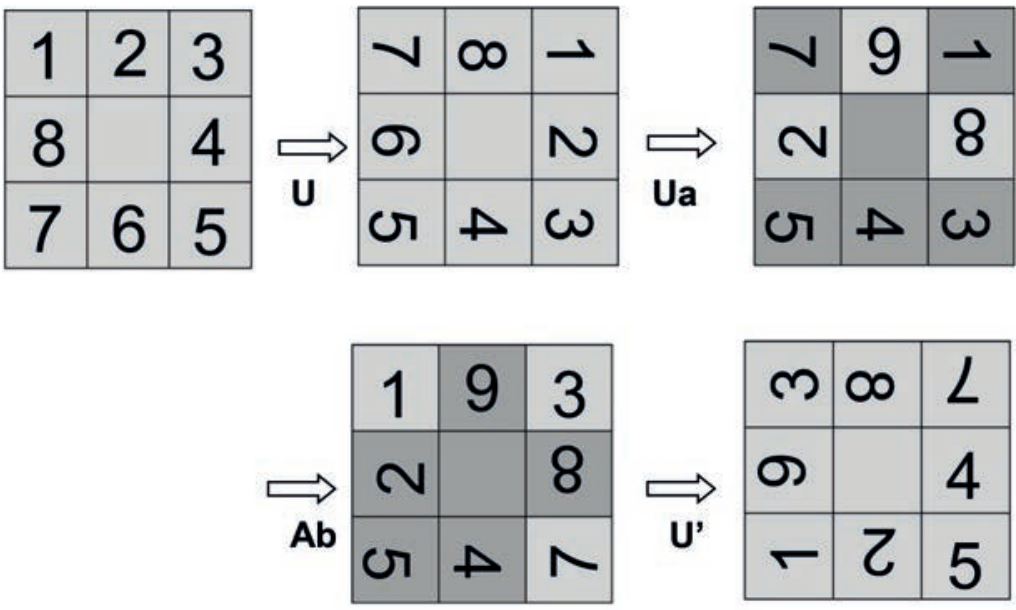

En síntesis:

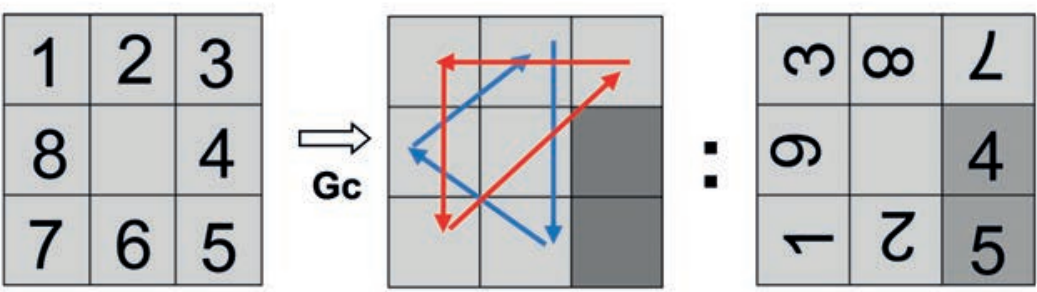

5.6.2 Objetivo de Gc (en la capa superior)

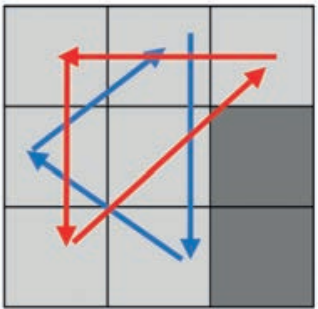

Gc

Permutar en sentido horario las tres aristas distintas a la derecha y, permutar en sentido antihorario los tres vértices distintos al inferior izquierdo. 


\subsubsection{Forma canónica del PLL “Gc"}

\section{F2 D R' U R' U' R D' F2 L' U L}

Con el cubo en su estado original y ejecutando:

$\mathrm{Gd}=\mathrm{Gc}^{-1}=\mathrm{RUR}^{\prime} \quad \mathrm{F} 2 \mathrm{D}^{\prime} \mathrm{L}$ U' $\quad$ L' U L' D $\quad$ F2 surge la configuración dada en la figura de arriba a la derecha. Al aplicar a esta configuración el algoritmo Gc se orienta todo el cubo (en este caso en especial se vuelve al cubo en su estado original).

\subsubsection{Configuración y Ayuda aplicativa de la permutación} "Gc"

- Hay uno y sólo una cara lateral donde en su lado de arriba se observa un "bloque de dos sticker contiguos "a izquierda" del mismo color" (opuesto al del sticker lateral del vértice que se halla en dicho lado). Ese lado de arriba se ubica en la cara derecha del cubo (no importa cómo quede el resto del cubo).

- Al colocar en la cara derecha el lado antes descrito, se observa en la cara frontal que "los tres stickers del lado superior son de colores distintos" y los de los primeros de derecha a izquierda $(\leftarrow)$ "no son opuestos"

- De tener una configuración en la capa superior como la descrita en los dos párrafos anteriores se aplica la permutación Gc. Al final con movimientos U se organiza totalmente el cubo. 
Síntesis tabular "Canónica” (Capítulo 5)

Para practicar y memorizar: 1 OLL Y 4 PLL

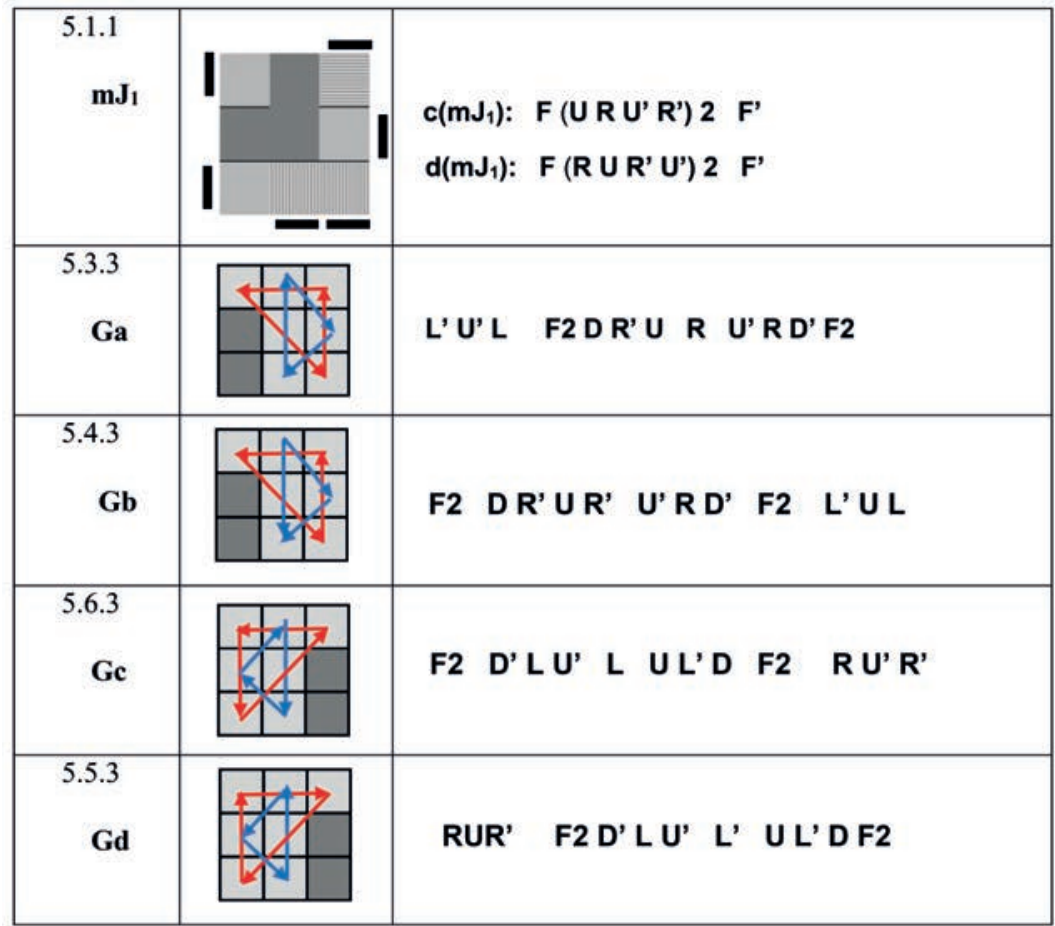


6 CAPÍTULO $_{\text {SEIS }}$ 


\section{Los Restantes 5 PLL}

Hasta este momento hemos tratado 16 PLL: 7 en el capítulo segundo, 5 en el cuarto y 4 en el capítulo anterior. Bien, del tratamiento de la primera Mini J, con otros algoritmos desde luego, surgen los restantes 5 del famoso grupo de "Los 21 clásicos PLL”.

\subsection{Las permutaciones " $R$ "}

Aprovechando el conocimiento de los PLL "J" y el reciente de las $4 \mathrm{G}$ seguidamente estudiamos las "R", Estas permutaciones tienen algo de parecido a la "J" en cuanto a que permutan vértices contiguos y aristas contiguas, pero difieren en que ninguna de las aristas a permutar está ubicada en el lado de los vértices a permutar; y en su configuración tienen algo de parecidas a las $G$ en cuanto a que poseen un único bloque de dos stickers juntos del mismo color.

\subsubsection{La “ $R_{D}$ ” o Primera R.}

- Motivación: $J_{\mathrm{I}}$ Gb U2 = “ $\mathrm{R}_{\mathrm{D}}$ ”

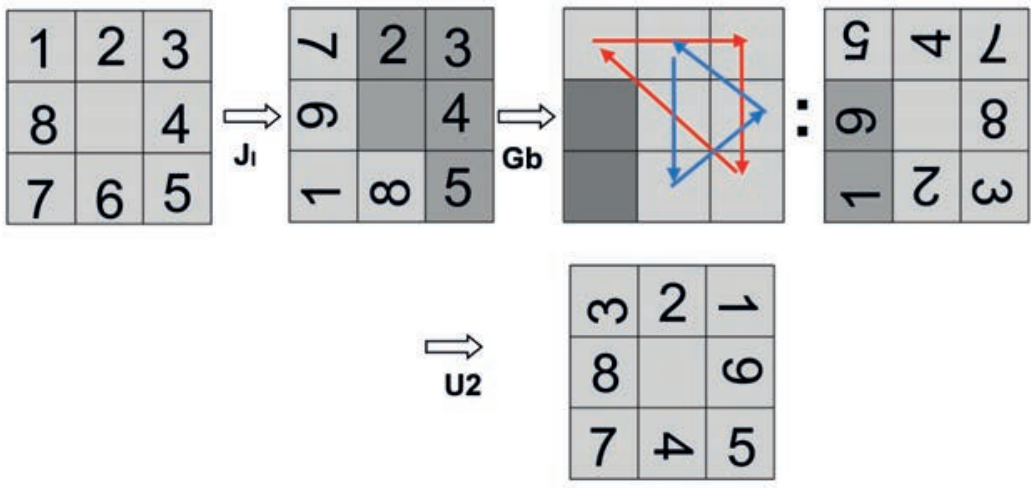

En síntesis: 

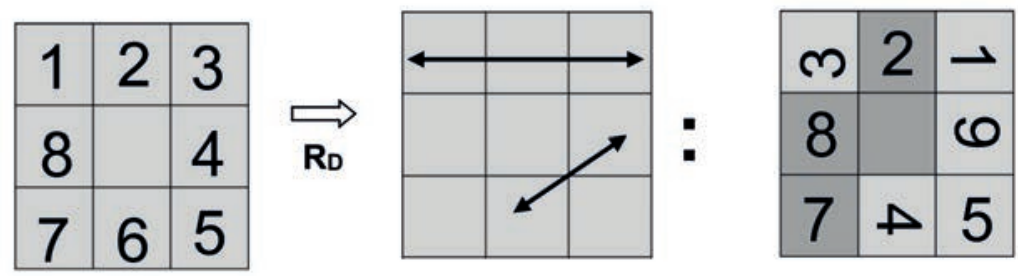

- Forma canónica del PLL " $R_{D}$ ”

(Permutación de los dos vértices superiores

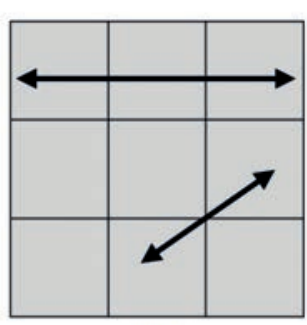

$\mathbf{R a}$ y también las aristas diagonales inferior y derecha).

\section{R' U2 R U2 R' FRU R' U'R' F' R2 U'}

Con el cubo en su estado original $\mathrm{y}$ ejecutando " $R_{D}$ " surge esa configuración. Una vez hecho " $\mathbf{R}_{\mathrm{D}}$ ” para volver al cubo en su estado original debe repetir tal ejecución

\subsubsection{La " $R_{I}$ ” o Simétrica de la Primera $R$}

- Motivación: $J_{\mathrm{D}}$ Gc U2 = "R "
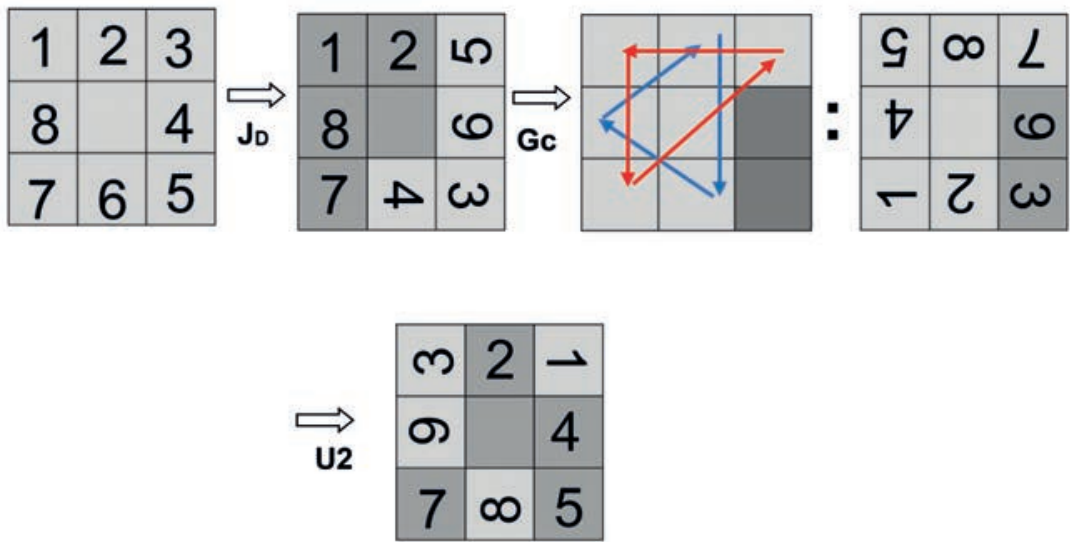

En síntesis: 

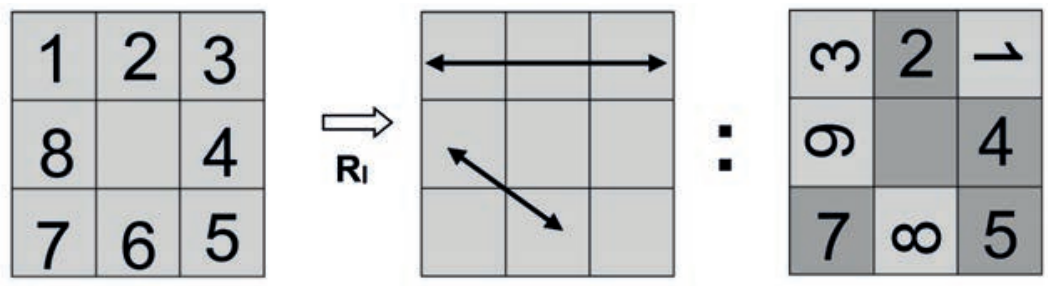

- Forma canónica del PLL "R"

(Permutación de los dos vértices superiores

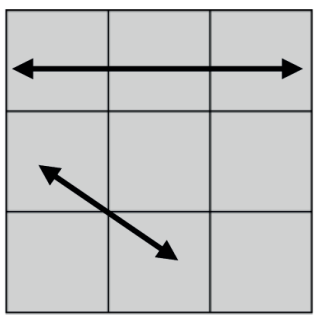

$\mathbf{R}$ y también las aristas diagonales inferior e izquierda).

\section{U2 L' U2 L F' L' U' L U L F L2 U}

Con el cubo en su estado original y ejecutando " $\mathbf{R}_{\mathrm{I}}$ " surge esa configuración. Una vez hecho " $\mathbf{R}_{\mathrm{I}}$ " para volver al cubo en su estado original debe repetir tal ejecución

\subsubsection{Configuraciones para aplicar los algoritmos " $R$ "}

- Las configuraciones para aplicar " $\mathrm{R}$ " son parecidas a las "G" en cuanto se refiere a las aristas contiguas a la inferior, pero difieren ya en lo relacionado con los vértices inferiores:

- De una parte, en las "R" los stickers frontales de esos vértices tienen el mismo color (lo que las hace diferentes de las configuraciones $\mathrm{Gb}$ y Gc donde son de color opuesto) y de otra parte, ese color no es el opuesto al del sticker frontal de la arista inferior (como sucede en Ga y $\mathrm{Gd)}$.

- Teniendo claro lo anterior precisamos las configuraciones " $\mathrm{R}_{\mathrm{D}}$ ” $\mathrm{y}$ “ $\mathrm{R}_{\mathrm{I}}$. En ambos casos nos concentramos únicamente en la capa superior, especialmente en los lados de arriba de las caras laterales. 


\subsubsection{Configuración y Ayuda aplicativa de " $R_{D}$ "}

- Hay una y sólo una cara lateral donde en su lado de arriba se observa un "único bloque de dos sticker contiguos "a derecha" del mismo color" ${ }^{\prime 2}$. Ese lado de arriba se ubica en la cara izquierda del cubo (no importa cómo quede el resto del cubo).

- Al colocar en la cara "izquierda" el lado antes descrito, observamos en el lado superior de la cara frontal que sus tres sticker alternan de modo ajedrezado en dos colores

- De tener una configuración en la capa superior como la descrita en los dos párrafos anteriores se aplica la permutación $\mathrm{R}_{\mathrm{D}}$ (Independiente de cómo sea el resto del cubo). Al final con movimientos $U$ se organiza totalmente el cubo.

\subsubsection{Configuración y Ayuda aplicativa de " $R_{I}$ "}

- Hay una y sólo una cara lateral donde en su lado de arriba se observa un "bloque de dos sticker contiguos "a izquierda" del mismo color" (opuesto al del sticker derecho del vértice que se halla en dicho lado). Ese lado de arriba se ubica en la cara derecha del cubo (no importa cómo quede el resto del cubo).

- Al colocar en la cara derecha el lado antes descrito, observamos en el lado superior de la cara frontal que sus tres sticker alternan de modo ajedrezado en dos colores.

- De tener una configuración en la capa superior como la descrita en los dos párrafos anteriores se aplica la permutación $\mathrm{R}_{\mathrm{I}}$ (Independiente de cómo sea el resto del cubo). Al final con movimientos $U$ se organiza totalmente el cubo.

22 Los R y los G. son los únicos PLL donde se observan esos únicos bloques de dos stickers laterales del mismo color 
Actividad (para el lector). Otra forma Constructiva de las " $R$ " usando " $U$ " $y$ "A"

únicamente. Usando el cubo de Rubik comprobar la validez de las siguientes igualdades:

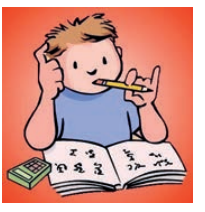

$$
\begin{aligned}
& R_{D}=U^{\prime} A b U 2 ~ U b \\
& R_{I}=U(A b)^{S} U 2 ~ U a
\end{aligned}
$$

\subsection{Otro constructor de $\mathrm{mJ}_{1}$. Inicio de los PLL “T” $\mathrm{y}$ “F”}

\subsubsection{Motivación}

$\mathbf{m J}_{1}=\mathrm{I}_{1}+\mathrm{U} 2+\mathrm{T}_{2}$. Apliquemos U2 y luego el constructor canónico de $\mathrm{T}_{2}$ (según lo expuesto en 2.1 .3 ) ${ }^{23}$ a la $\mathrm{I}_{1}$ de más abajo.
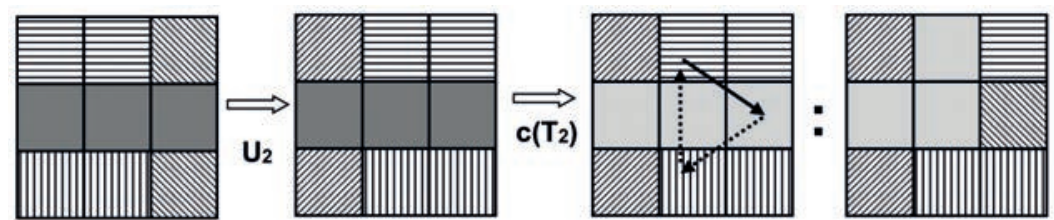

$\mathbf{l}_{1}$
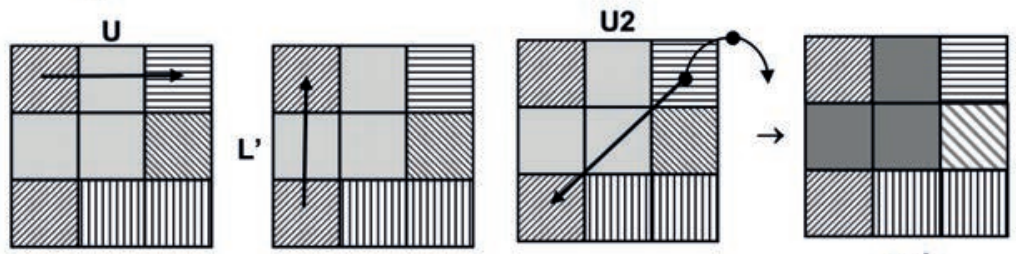

$\mathrm{mJ}_{1}$

\subsubsection{Nuevo constructor/deconstructor de $\mathrm{mJ}_{1}$ o $\mathrm{mJ}(2,1,1,2)$}
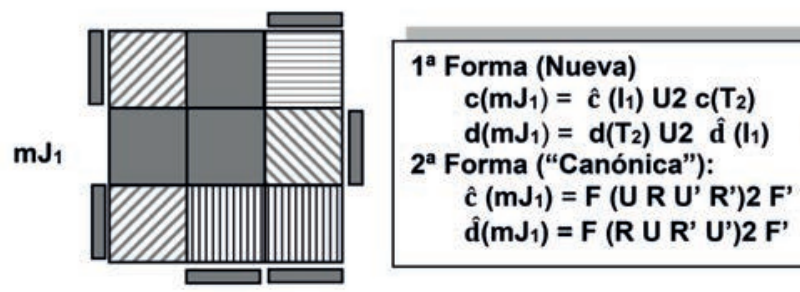

23 Recordar. Este constructor actúa sobre el vértice superior derecho del modo siguiente: le da una torsión horaria de tres giros y luego lo rota $180^{\circ}$. 
(*)

ć(I) U2 c(T2) = F (R U R' U')2 F' U2 (F R' F' R U R U' R')

d(T $\left.T_{2}\right)$ U2 $\hat{d}\left(I_{1}\right)=(R$ U R' U' R' F R F') U2 F(U R U' R')2 F',

\subsubsection{La no congruencia de las dos formas}

Una diferencia radica en los vértices de la cara superior: Con la $2^{\text {a }}$ forma todos los vértices quedan bien posicionados, cosa que no se da con la $1^{\text {a }}$ forma.

6.2.4 La semejanza en la configuración de las dos formas.

$\mathrm{Al}$ ejecutar los constructores de ambas formas se obtiene la misma configuración por semejanza:

$$
\widetilde{\mathrm{C}_{\mathrm{p}}}=\widetilde{\mathrm{C}_{\mathrm{q}}}
$$

siendo $\mathrm{p}=\mathrm{c}\left(\mathrm{mJ}_{1}\right)=\hat{\mathbf{c}}\left(\mathbf{I}_{1}\right) \mathbf{U} \mathbf{2}\left(\mathbf{T}_{2}\right) \quad$ y $\quad \mathrm{q}=\hat{\mathbf{c}}\left(\mathbf{m J _ { 1 }}\right)=\mathbf{F}\left(\mathbf{U} \mathbf{R} \mathbf{U}^{\prime} \mathbf{R}^{\prime}\right) \mathbf{2}$ F',

A continuación, se verificará lo anterior también de modo algebraico Intercambiando constructor/deconstructor.

6.2.4.1 Situación 1.p: $\mathrm{c}\left(\mathrm{mJ}_{1}\right) \hat{\mathbf{d}}\left(\mathrm{mJ}_{1}\right)$ donde $\mathbf{c}\left(\mathbf{m J}_{1}\right)=\hat{\mathbf{c}}\left(\mathbf{I}_{1}\right) \mathbf{U} \mathbf{2} \mathbf{c}\left(\mathbf{T}_{2}\right)$ y $\hat{\mathbf{d}}\left(\mathbf{m J}_{1}\right)=\mathbf{F}\left(\mathbf{R} \mathbf{U} \mathbf{R}^{\prime} \mathbf{U}^{\prime}\right) \mathbf{2}$ F'

Sea $C_{p}$ la configuración resultante de ejecutar $\mathbf{p}$ desde el cubo en su estado original. Sucede que $\mathrm{C}_{\mathrm{p}}=\mathrm{C}_{\text {T.o. }}$ (cubo totalmente orientado, pero no totalmente organizado).

$$
\mathbf{c}\left(\mathbf{m J}_{\mathbf{1}}\right) \hat{\mathbf{d}}\left(\mathbf{m J}_{\mathbf{1}}\right) \longrightarrow \mathbf{C}_{\text {T.O }}
$$

Para llevar el cubo a su estado original puede aplicarse el PLL: U' T U2

$$
c\left(m J_{1}\right) \hat{d}\left(m^{\prime} J_{1}\right) \underset{U^{\prime} T U 2}{\longrightarrow} c_{1}
$$

donde $\mathbf{T}=\mathbf{U}^{\prime} \mathbf{A b} \mathbf{U Z Z}$ Ab o simplemente $\mathbf{T}=\mathbf{F} \mathbf{A b}$ $\operatorname{con} \mathbf{F}=\mathbf{U}^{\prime} \mathbf{A b} \mathbf{U Z U}$. 


\subsubsection{Situación 2. q: $\widehat{\mathrm{c}}\left(\mathrm{mJ}_{1}\right) \mathrm{d}\left(\mathrm{mJ}_{1}\right)$}

donde $\hat{\mathbf{c}}\left(\mathbf{m} \mathbf{J}_{1}\right)=\mathbf{F}\left(\mathbf{U} \mathbf{R} \mathbf{U}^{\prime} \mathbf{R}^{\prime}\right) \mathbf{2} \mathbf{F}^{\prime}$ y $\mathbf{d}\left(\mathbf{m J}_{1}\right)=\mathbf{d}\left(\mathbf{T}_{2}\right) \mathbf{U} \mathbf{2}$ d̂ $\left(\mathbf{I}_{1}\right)$.

El lector puede comprobar la validez del esquema siguiente:

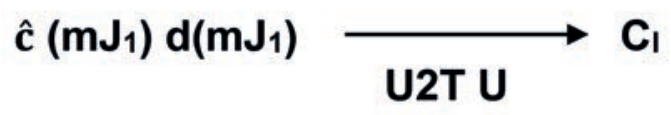

donde $\mathbf{T}=\mathbf{F} \mathbf{A b}$ con $\mathbf{F}=\mathbf{U}^{\prime} \mathbf{A b} \mathbf{U Z Z}$.

\subsection{La permutación "F"}

6.3.1 Motivación: U’ Ab UZU = “F”

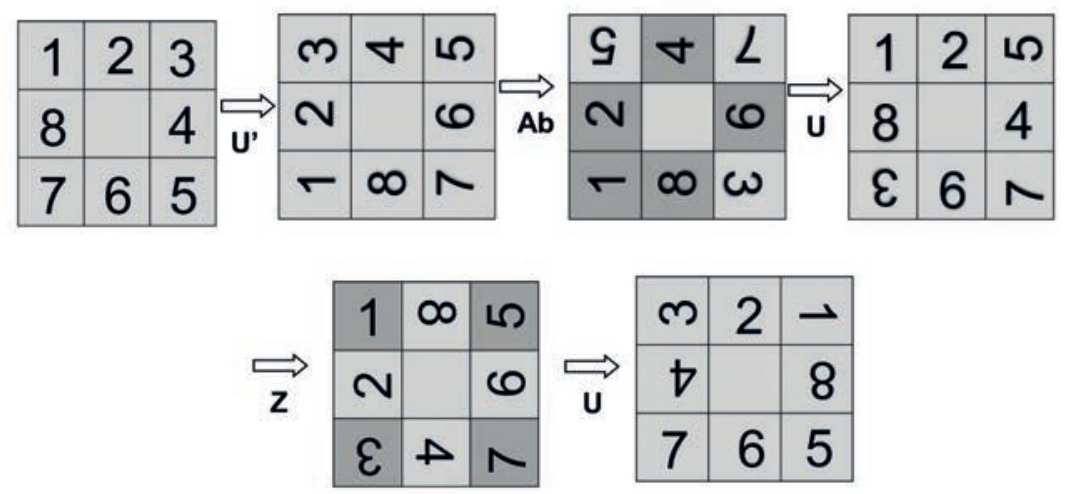

En síntesis:
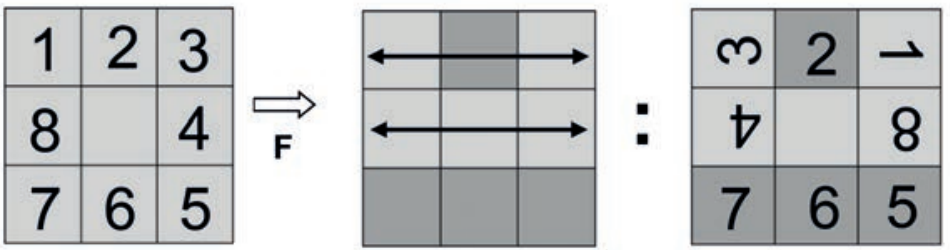

6.3.2 Objetivo de la "F"

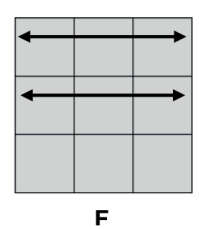

Esta permutación lo que hace es intercambiar los dos vértices superiores y a la vez intercambia las aristas izquierda y derecha 


\subsubsection{Forma canónica del PLL “F”}

\section{(R' U R U') R2 (F'U'FU RFR'F') R2 U'}

Con el cubo en su estado original y ejecutando "F" surge esa configuración. Una vez hecho " $F$ " para volver al cubo en su estado original debe repetir tal ejecución.

\subsubsection{Configuración y Ayuda aplicativa de la permutación} "F"

- Nos concentramos únicamente en la capa superior, especialmente en los lados de arriba de las caras laterales (las otras dos capas están ya organizadas y no nos interesan mucho en este momento).

- Existe uno y solo un lado de arriba totalmente organizado (todos sus stickers son del mismo color). Colocamos ese lado en la cara frontal.

- En cada uno de los lados contiguos al antes mencionado hay un bloque de dos sticker juntos de color opuesto; los colores de los stickers en ambos bloques son los mismos pero intercalados.

- Una vez examinadas estas características y ratificadas que se dan, se coloca frontalmente el lado completamente organizado (no interesa como quede el resto del cubo) y se aplica el algoritmo "F". Al final con movimientos U se organiza totalmente el cubo.

\subsection{La permutación “T”}

\subsubsection{Motivación: $\mathrm{F} \mathrm{Ab}=$ “ $\mathrm{T}$ ”}

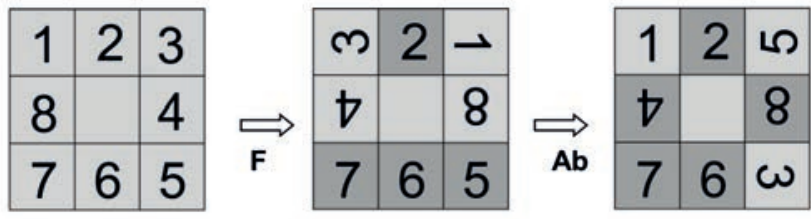

\section{En síntesis:}




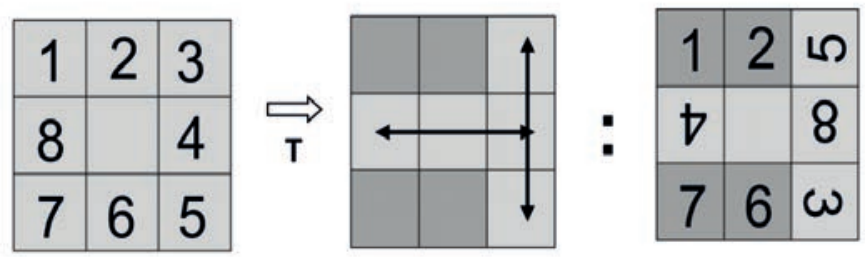

6.4.2 Objetivo de la " $T$ ”

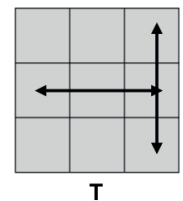

Esta permutación lo que hace es intercambiar los dos vértices derechos y a la vez intercambia las aristas izquierda y derecha.

\subsubsection{Forma canónica del PLL “T”}

\section{RU R' U' R' FR2 U'R' U'RU R'F'}

Con el cubo en su estado original y ejecutando "T" surge esa configuración. Una vez hecho "T" para volver al cubo en su estado original debe repetir tal ejecución.

\subsubsection{Configuración y ayuda aplicativa de la permutación} "T"

- Nos concentramos únicamente en la capa superior, especialmente en los lados de arriba de las caras laterales (las otras dos capas están ya organizadas y no nos interesan mucho en este momento).

- Se observan dos bloques y únicamente dos constituidos por dos sticker juntos del mismo color. Estos bloques están en lados opuestos y sus colores también son opuestos.

- Los stickers restantes (uno por cada lado) que acompañan a tales bloques son del mismo color. Ubicamos esos stickers en el lado derecho de la capa superior; Así "uno de los bloques" mencionados en el párrafo anterior "queda frontalmente y a la "izquierda"” de uno de dichos stickers. 
- Una vez examinadas estas características y ratificadas que se dan, se aplica el algoritmo "T". Al final con movimientos $\mathrm{U}$ se organiza totalmente el cubo.

A continuación, y aprovechando esta última permutación $\mathrm{T}$ y la $\mathrm{J}_{\mathrm{I}}$ damos lugar a una interesante permutación como es la " $\mathrm{Y}$ "

\subsection{La permutación "Y"}

6.5.1 Motivación: U J T $=$ "Y"24

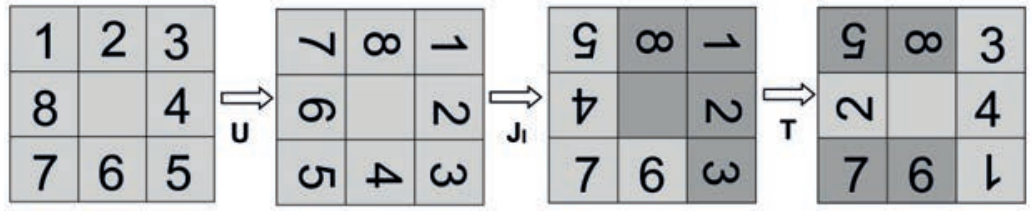

En síntesis:
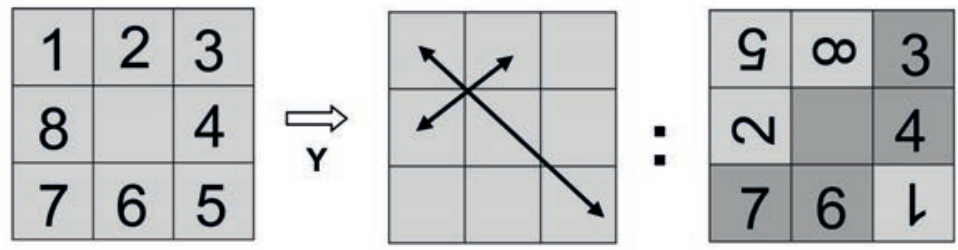

\subsubsection{Objetivo de la "Y"}

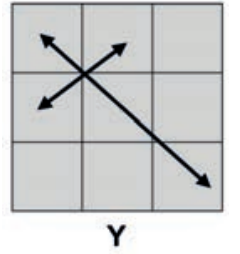

Esta permutación lo que hace es intercambiar los vértices que están en la diagonal NO principal (superior izquierdo e inferior derecho) y a la vez intercambia las aristas diagonales contiguas al vértice superior izquierdo (aristas superior e izquierda).

\subsubsection{Forma canónica del PLL "Y"}

$$
\begin{aligned}
& F R\left(U^{\prime} R^{\prime} U^{\prime}\right)\left(R U^{\prime} R^{\prime}\right) d\left(T_{2}\right) \\
& d\left(T_{2}\right)=\left(R R^{\prime} U^{\prime}\right)\left(R^{\prime} F\right. \text { R F') }
\end{aligned}
$$

$24 \mathrm{~J}_{\mathrm{I}}=\mathrm{B}^{\prime}$ U B' R2 F D' F D $\quad$ F2 R2 B2, 4.4.2 
Con el cubo en su estado original y ejecutando "Y" surge esa configuración. Una vez hecho "Y" para volver al cubo en su estado original debe repetir tal ejecución.

\subsubsection{Configuración y Ayuda aplicativa de la permutación "Y"}

- Esta configuración tiene algo de parecido con la configuración de las "N" en el sentido que existe un par de lados superiores de las caras laterales (a la superior) con un bloque de dos stickers juntos del mismo color, pero la diferencia radica en que cuando se trata de la "N" esto se observa en cada lado superior de las caras laterales a la superior, en tanto que en la "Y" esto se da únicamente en dos lados contiguos.

- Nos concentramos únicamente en la capa superior, especialmente en los lados de arriba de las caras laterales.

- Hay un par de lados contiguos y sólo un par donde en cada uno de ellos se observa un bloque de dos stickers laterales (no superiores) del mismo color en tanto que el color del tercer sticker lateral (del vértice restante) tiene color opuesto al así exhibido en ese bloque. Esos dos bloques ${ }^{25}$ están separado por un vértice que bien podríamos llamar "Vértice de interés para Y"; los stickers laterales de ese vértice son de colores opuestos al de los dos bloques que comunica.

- Ubicamos el "vértice de interés para Y" como vértice inferior derecho de la capa superior.

- De tener una configuración de ese tipo en la capa superior se aplica el algoritmo "Y".

25 Esos bloques están orientados de modo opuesto: Uno de ellos tiene los stickers juntos "a derecha" en tanto que el otro tiene sus sticker juntos "a izquierda". 
Síntesis tabular "Canónica" (Capítulo 6)

Para practicar y memorizar: 5 PLL

\begin{tabular}{|c|c|c|}
\hline $\begin{array}{r}6.1 .1 \\
\text { RD }\end{array}$ & $\longleftrightarrow$ & FRU R' U'R' F' R2 U' \\
\hline $\begin{array}{r}6.1 .2 \\
\mathbf{R}_{\mathbf{I}}\end{array}$ & & L U2 L'U2 L F'L'U' L ULFL2U \\
\hline $\begin{array}{r}6.3 .2 \\
\mathbf{F}\end{array}$ & & (R' U R U') R2 (F'U'FU RFR'F') R2 U' \\
\hline $\begin{array}{r}6.4 .2 \\
T\end{array}$ & & FR2 U'R' U'RU R'F' \\
\hline $\begin{array}{r}6.5 .2 \\
\mathbf{Y}\end{array}$ & & F R (U' R' U') (R U R' F') (R U R'U' R'F R F') \\
\hline
\end{tabular}




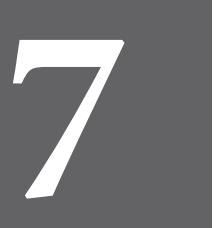

CAPÍTULO SIETE 


\section{La primera Mini L y otros. . . Cuatro de "Cruz Ampliada"}

La primera Mini L, notada Mini L1 o Mini L $(2,2,1,1)$, es la simétrica de la mini $\mathrm{J}_{1}$ tratada en el capítulo anterior; y junto con esta se constituyen en algoritmos, como veremos en este y en la parte II de este trabajo en otro libro, muy usados para construir/ deconstruir configuraciones en las cuales se pueda distinguir una Mini L como el caso de las "Cruces ampliadas" y en otras que llamaremos las "Mini L ampliadas"26
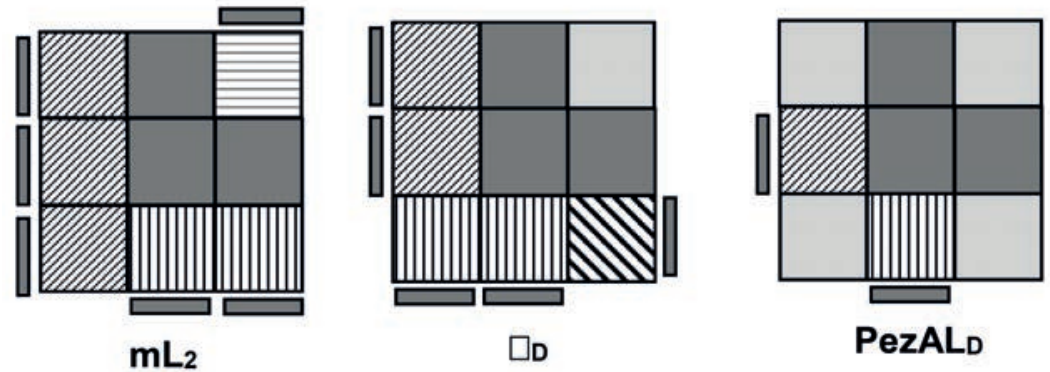

Uno de los objetivos de este capítulo es permitir que el lector se familiarice un poco más con lo simétrico haciendo que lo realizado con la derecha se practique con la izquierda obteniendo nuevos y exitosos resultados. Es así como haremos un trabajo simétrico con partes del capítulo anterior para la, Mini $\mathrm{L}_{1}$, simétrico de Mini $\mathrm{J}_{1}$; practicando de paso varios de los PLL allí exhibidos.

Jugando con estas Mini obtendremos unos nuevos OLL, un objetivo más del capítulo. De modo preciso damos a conocer otros cuatro del grupo de las "7 cruces ampliadas": "La Cruz, el camaleón, la Pajarita y la botella." Ya en el capítulo 4 habíamos hablado dela Cruz $z_{1}$, perteneciente a dicho grupo, cuya característica fundamental es la de cada uno de sus siete integrantes tiene "todas tus aristas bien orientadas."

26 Estas "Mini L ampliadas" No son "cruces ampliadas". Algo más, no es recomendable usar "Mini $\mathrm{L}_{1}$ ” para deconstruir tales cruces ampliadas. 
- En este capítulo y en adelante, se va a usar la configuración adición de otras dos (3.1.3), $\mathrm{A}=\mathrm{B}+\mathrm{C}$, a partir de los constructores canónicos de $\mathrm{B}$ y $\mathrm{C}$ :

\section{$\mathbf{A}=\mathbf{B}+\mathbf{C}$ significa que $\hat{\boldsymbol{c}}(\mathbf{B}) \hat{\boldsymbol{c}}(\mathbf{B})$ es un constructor de $\mathbf{A}$} $\mathrm{k}, \mathrm{m}=0,1,2,3$.

\section{$7.1 \mathrm{~mL}_{1}$ : La Primera Mini $\mathrm{L}(\mathrm{mL}(2,2,1,1))$ o Simétrica de $\mathrm{mJ}_{1}$}

\subsubsection{Motivación:}

$\mathbf{m L}_{1}=\mathrm{T}_{1} \mathrm{~s}+\mathrm{T}_{1} \mathrm{~s}$. Apliquemos el constructor canónico de $\mathrm{T}_{1}^{\mathrm{S}}$ (según lo expuesto en 1.8.3) a la $\mathrm{T}_{1}^{\mathrm{S}}$ de más abajo $\left(=\mathrm{c}\left(\mathrm{T}_{1}\right)^{\mathrm{S}}\right)$
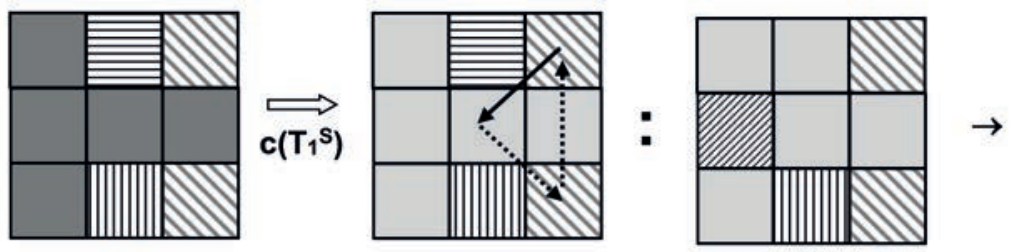

$T_{1} s$

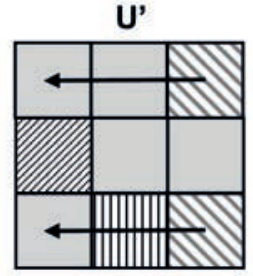

$\mathbf{U}$

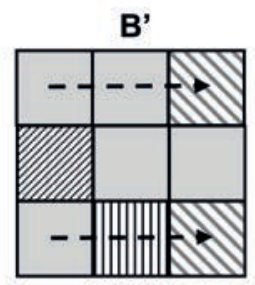

$\mathbf{F}$

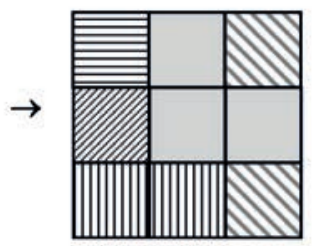

$\mathrm{mL}_{1}$

7.1.2 Constructor/deconstructor canónicos de “ $\mathrm{mL}_{1}$ ” (= $\mathrm{mJ}_{1}{ }^{\mathrm{S}}$ )

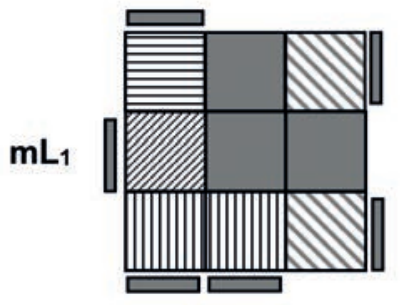

$$
\begin{aligned}
& \hat{\mathbf{c}}\left(\mathrm{mL}_{1}\right)=F^{\prime}\left(U^{\prime} L^{\prime} U^{\prime} L^{\prime}\right) 2 \mathrm{~F} \\
& \hat{\mathbf{d}}\left(\mathrm{m}_{1}\right)=F^{\prime}\left(L^{\prime} U^{\prime} L U\right) 2 \mathrm{~F}
\end{aligned}
$$




\subsubsection{Dinámica del $\widehat{c}\left(\mathrm{~mL}_{1}\right)$ en la capa superior}
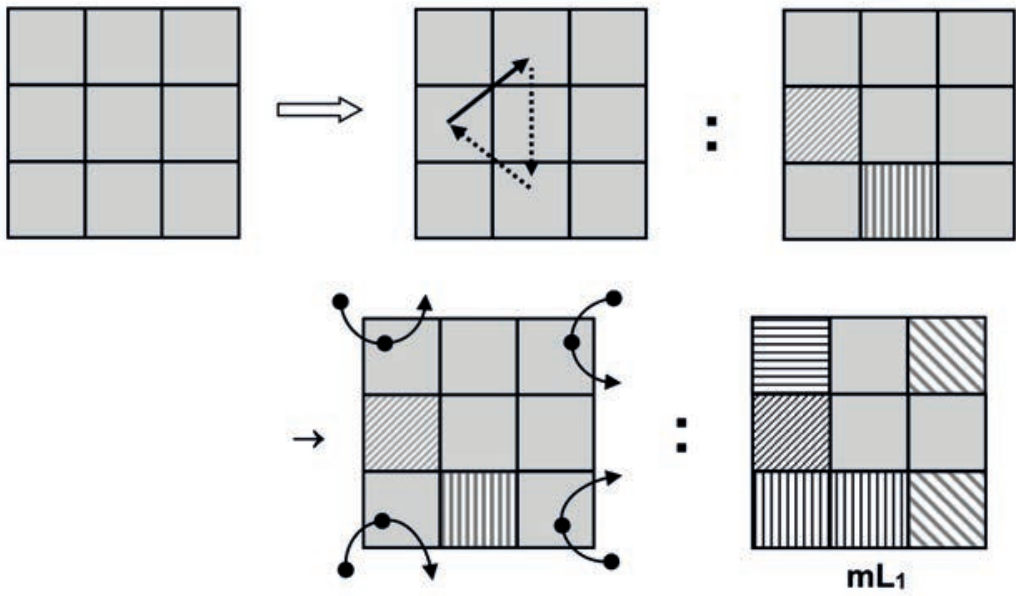

\subsubsection{Acción de $\widehat{c}\left(\mathrm{~mL}_{1}\right)$ sobre sus aristas iniciales}

$\hat{\mathbf{c}}\left(\mathbf{m L}_{\mathbf{1}}\right)$ realiza una permutación en sentido antihorario de tres de las aristas distintas de la derecha (permanece invariante), provocando permutaciones en los stickers de algunas de estas. De modo preciso:

- La arista izquierda pasa a ser la arista superior;

- La arista superior pasa a ser la inferior y sus stickers permutan;

- La arista inferior pasa a ser la derecha y sus stickers permutan.

\subsubsection{Acción de $\hat{\mathbf{c}}\left(\mathrm{mL}_{1}\right)$ sobre sus vértices iniciales.}

- Los vértices frontales (inferior izquierdo e inferior derecho) reciben una torsión "horaria" de tres giros (1.6.3.3).

- Los vértices de atrás (superior izquierdo y superior derecho) reciben una torsión "antihoraria" de tres giros (1.6.3.3). 
7.2 Nuevos constructores/ deconstructores de $\mathrm{mL}_{1}$

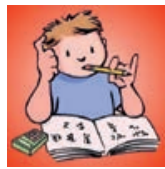

Esta sección la debe desarrollar activamente el lector por su propia cuenta con la información acá consignada.

7.2.1 Constructor/deconstruir de $\mathrm{mL}_{1}$ a partir de $\mathrm{I}_{1} \mathrm{y} \mathrm{T}_{2} \mathrm{~S}$. Sean:

$$
\begin{aligned}
& \mathrm{c}\left(\mathrm{mL}_{1}\right)=\mathbf{c}\left(\mathbf{I}_{1}\right) \mathbf{c}\left(\mathbf{T}_{2}{ }^{\mathbf{S}}\right)=\mathbf{F}\left(\mathbf{R U} \mathbf{R}^{\prime} \mathbf{U}^{\prime}\right) 2 \text { F' F' LFL' U'L' U L }
\end{aligned}
$$

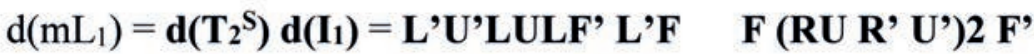

Usando el cubo de Rubik en su estado original comprobar la veracidad de los siguientes diagramas

$$
\begin{aligned}
& \mathbf{c}\left(m L_{1}\right) \hat{\mathbf{d}}\left(m L_{1}\right) \underset{\mathbf{U}^{\prime} \mathbf{G d ~ U}}{\longrightarrow} \mathbf{C}_{\mathbf{I}} \\
& \hat{\mathbf{c}}\left(m L_{1}\right) \mathbf{d}\left(m L_{1}\right) \underset{\mathbf{U}^{\prime} \mathbf{G c ~ U}}{\longrightarrow} \mathbf{C}_{\mathbf{I}}
\end{aligned}
$$

donde $\hat{\mathbf{c}}\left(\mathbf{m L}_{1}\right)=\mathrm{F}^{\prime}\left(\mathrm{U}^{\prime} \mathrm{L}^{\prime} \mathrm{UL}\right) 2 \mathrm{~F} \quad \hat{\mathrm{d}}\left(\mathbf{m L}_{\mathbf{1}}\right)=\mathrm{F}^{\prime}\left(\mathrm{L}^{\prime} \mathrm{U}^{\prime} \mathrm{L}^{\prime} \mathrm{U}\right) 2 \mathrm{~F}$

$$
\text { Gd = RUR' F2 D' L U' L' U L' D F2 (5.5.3) }
$$

$$
\mathbf{G c}=\text { F2 D' L U' L U L' DF2 R U'R' (5.6.3) }
$$

7.2.2 Constructor/deconstruir de $\mathrm{mL}_{1}$ a partir de $\mathrm{I}_{1}{ }^{\mathrm{s}} \mathrm{y} \mathrm{T}_{2} \mathrm{~s}^{\mathrm{s}}$ : Sean

$$
\begin{aligned}
& \mathrm{c}\left(\mathrm{mL}_{1}\right)=\mathbf{c}\left(\mathrm{I}_{1}^{\mathrm{S}}\right) \mathbf{U} 2 \mathbf{c}\left(\mathbf{T}_{2}{ }^{\mathrm{S}}\right)=\mathrm{F}^{\prime} \text { (L'U' L U)2 F U2 F' LFL' U'L' U L }
\end{aligned}
$$

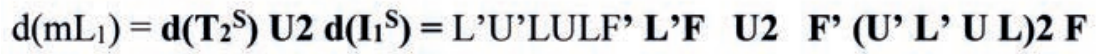

Usando el cubo de Rubik en su estado original comprobar la veracidad de los siguientes diagramas

$$
\begin{aligned}
& \mathbf{c}\left(m L_{1}\right) \hat{\mathbf{d}}\left(m L_{1}\right) \underset{\mathbf{U}^{\prime} \mathbf{T}}{\longrightarrow} \mathbf{C}_{1} \\
& \hat{\mathbf{c}}\left(m L_{1}\right) \mathbf{d}\left(m L_{1}\right) \underset{\text { T U }}{\longrightarrow} \mathbf{C}_{1}
\end{aligned}
$$


donde $\hat{\mathbf{c}}\left(\mathbf{m L}_{\mathbf{1}}\right)=\mathrm{F}^{\prime}\left(\mathrm{U}^{\prime} \mathrm{L}^{\prime} \mathrm{U} \mathrm{L}\right) 2 \mathrm{~F}$

$$
\begin{aligned}
& \hat{\mathrm{d}}\left(\mathrm{mL}_{1}\right)=\mathrm{F}^{\prime}\left(\mathrm{L}^{\prime} \mathrm{U}^{\prime} \mathrm{L} \mathrm{U}\right) 2 \mathrm{~F} \\
& \text { T }=\text { RU R' U' R' FR2 U'R' U' RU R'F' (6.4.2) }
\end{aligned}
$$

El resto del capítulo se dedica a cinco configuraciones OLL del Grupo de "Las siete cruces ampliadas", cuatro de estas nuevas pues la primera que seguidamente analizamos, la $\mathrm{Cruz}_{1}$, ya apareció en las secciones 4.7.1 y 4.8. del cuarto capítulo.

7.3 Nuevo constructor/deconstructor de $\mathrm{Cruz}_{1}$ con la simétrica de $\mathrm{T}_{1}$ y la primera mini $\mathrm{L} . \mathrm{Cruz}_{1=} \mathrm{T}_{1}^{\mathrm{S}}+\operatorname{Mini} \mathrm{L}_{1}$

\subsubsection{Motivación:}

$\mathrm{Cruz}_{1}=\hat{\mathbf{c}}\left(\mathrm{T}_{1}{ }^{\mathrm{s}}\right) \hat{\mathbf{c}}\left(\right.$ Mini $\left.\mathrm{L}_{1}\right)$ : Apliquemos el constructor canónico de $\mathrm{mL}_{1}$ (según lo expuesto en 7.1.2) a la $\mathrm{T}_{1}{ }^{\mathrm{S}}$ de más abajo $\left(=\widehat{\mathbf{c}}\left(\mathrm{T}_{1}^{\mathrm{S}}\right)\right)$
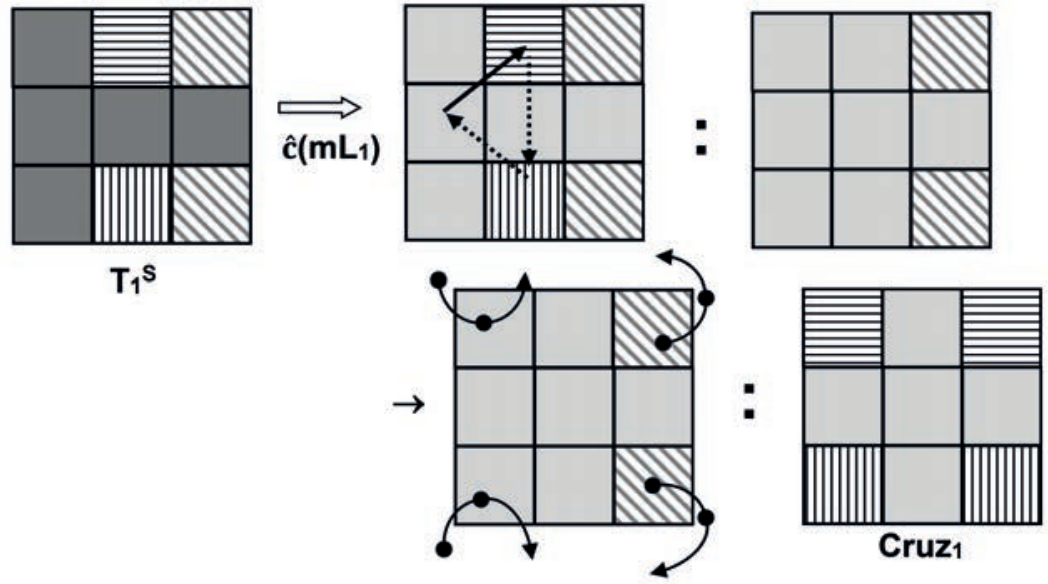

7.3.2 Nuevo constructor/deconstructor de la Cruz
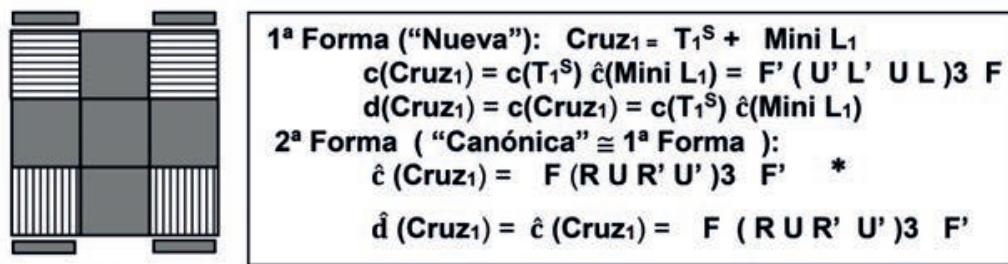

Cruz1 
* $\mathbf{c}\left(\mathbf{T}_{1}{ }^{\mathbf{S}}\right) \mathbf{c}\left(\right.$ Mini $\left.\mathbf{L}_{1}\right)=\left(F^{\prime} \mathrm{U}^{\prime} \mathrm{L}^{\prime} \mathrm{U}\right.$ L F) F' (U' L' U L) $2 \mathrm{~F}=\mathrm{F}^{\prime}\left(\mathbf{U}^{\prime} \mathbf{L}^{\prime} \mathbf{U} \mathbf{L}\right) 3 \mathbf{~ F}$

Comentario: Estas dos formas son congruentes

$\mathbf{c}\left(\mathrm{Cruz}_{1}\right) \hat{\mathbf{d}}\left(\mathbf{C r u z}_{1}\right) \quad=\mathbf{C}_{\mathbf{l}}$ : Cubo en su estado inicial.

$\hat{\mathbf{c}}\left(\mathrm{Cruz}_{1}\right) \mathrm{d}\left(\mathrm{Cruz}_{1}\right)=\mathrm{Cl}$ : Cubo en su estado inicial.

7.4 La segunda Cruz: $\operatorname{Cruz}_{2}=\mathrm{I}_{1}+\mathrm{Mini} \mathrm{L}_{1}$

7.4.1 Motivación. $\mathrm{Cruz}_{2}=\widehat{\mathrm{C}}\left(\mathrm{I}_{1}\right) \widehat{\mathrm{C}}\left(\mathrm{Mini}_{1}\right)$
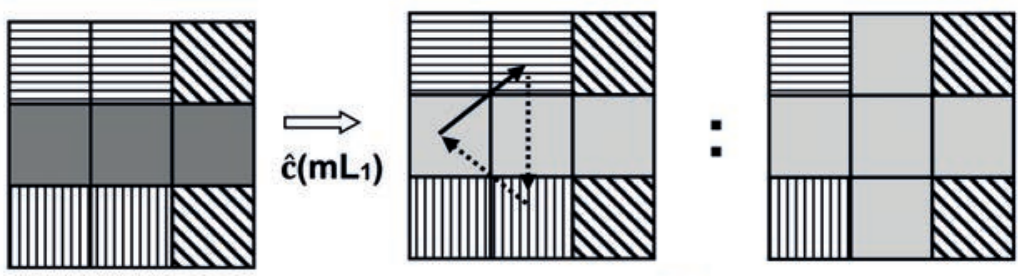

$l_{1}$
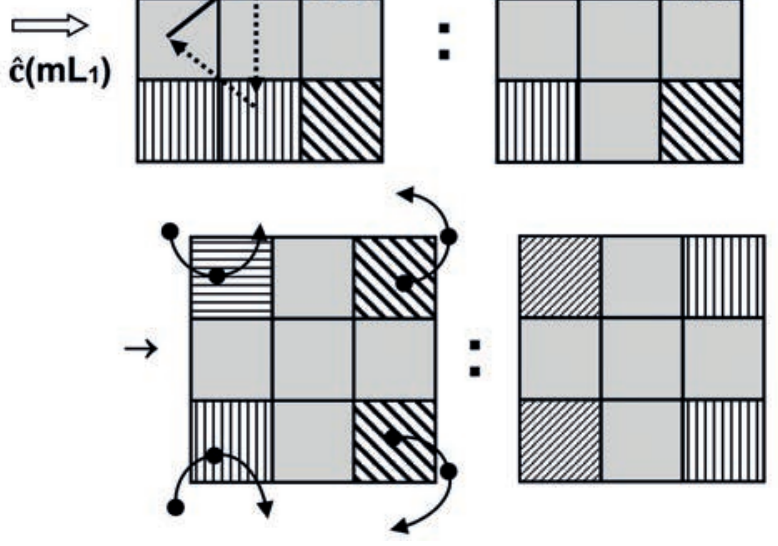

7.4.2 Dos formas de construir/deconstruir Cruz,

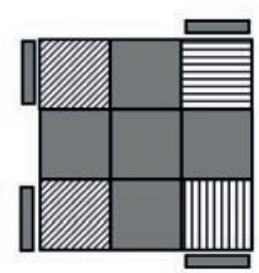

$1^{\text {a }}$ Forma: $\operatorname{Cruz}_{2}=I_{1}+$ Mini $L_{1}$

$$
\begin{aligned}
& c\left(\text { Cruz }_{2}\right)=\hat{c}\left(I_{1}\right) \hat{c}\left(\text { Mini } L_{1}\right) \\
& \mathbf{d}\left(\text { Cruz }_{2}\right)=\hat{d}\left(\text { Mini } L_{1}\right) \hat{d}\left(I_{1}\right)
\end{aligned}
$$

2a Forma("canónica"):

$\hat{\mathbf{c}}\left(\mathrm{Cruz}_{2}\right)=\mathbf{R}^{\prime} \mathbf{U} 2 \mathbf{R 2} \mathbf{U} \mathbf{R 2} \mathbf{U} \mathbf{R 2} \mathbf{U} 2 \mathbf{R}^{\prime}$

$\mathrm{Cruz}_{2}$

d̂(Cruz2) = R U2 R2 U' R2 U' R2 U2 R

(*) $\quad \hat{\mathbf{c}}\left(\mathbf{I}_{1}\right) \hat{\mathbf{c}}\left(\right.$ Mini $\left.\mathrm{L}_{1}\right)=\mathbf{F}($ R U R' U')2 F' F' (U' L' U L)2 F

d̂(Mini $\left.L_{1}\right)$ d̂(I $I_{1}=$ F' (L' U' L U)2 F F (U R U' R' )2 F', 


\subsubsection{La no congruencia de las dos formas}

En la configuración de la $2^{\text {a }}$ forma todos los vértices iniciales inferiores adquieren torsión horaria de tres giros y los superiores torsión antihoraria de tres giros; en tanto que con la primera sucede todo lo contrario: todos los vértices iniciales inferiores adquieren torsión antihoraria de tres giros y los superiores torsión horaria de tres giros.

7.4.4 La semejanza de la configuración de las dos formas: Intercambiando constructor/deconstructor.

7.4.4.1 Situación 1. p: $\mathbf{c}\left(\mathrm{Cruz}_{2}\right) \hat{\mathbf{d}}\left(\mathrm{Cruz}_{2}\right)$, donde $\mathrm{c}\left(\mathrm{Cruz}_{2}\right)=\hat{\mathrm{c}}\left(\mathrm{I}_{1}\right) \hat{\mathrm{c}}\left(\right.$ Mini L $\left._{1}\right)=\mathrm{F}\left(\mathrm{R} \mathrm{U} \mathrm{R}^{\prime} \mathrm{U}^{\prime}\right) 2 \mathrm{~F}^{\prime} \quad \mathrm{F}^{\prime}\left(\mathrm{U}^{\prime} \mathrm{L} \mathrm{L}^{\prime} \mathrm{U} \mathrm{L}\right) 2 \mathrm{~F}$

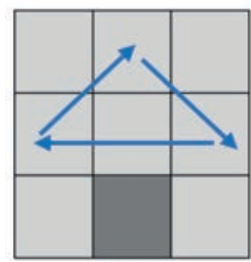

$\mathrm{Ua}$ $\widehat{d}\left(\mathrm{Cruz}_{2}\right)=\mathrm{R}$ U2 R2 U' R2 U' R2 U2 R

El lector puede comprobar la validez del esquema siguiente:

\section{$\mathrm{c}\left(\mathrm{Cruz}_{2}\right) \hat{\mathrm{d}}\left(\mathrm{Cruz}_{2}\right) \stackrel{\mathrm{U} 2 \mathrm{Ua}}{\longrightarrow} \mathrm{C}_{1}$}

7.4.4.2 Situación 2. q: $\left.\hat{\mathbf{c}} \mathrm{Cruz}_{2}\right) \mathbf{d}\left(\mathrm{Cruz}_{2}\right)$, donde $\widehat{\mathrm{c}}\left(\mathrm{Cruz}_{2}\right)=$ R' U2 R2 U R2 U R2 U2 R' $\mathrm{d}\left(\mathrm{Cruz}_{2}\right)=\hat{\mathrm{d}}\left(\right.$ Mini $\left._{\mathrm{L}}\right) \hat{\mathrm{d}}\left(\mathrm{I}_{1}\right)=\mathrm{F}^{\prime}\left(\mathrm{L}^{\prime} \mathrm{U}^{\prime} \mathrm{L} \mathrm{U}\right) 2 \mathrm{~F} \quad \mathrm{~F}\left(\mathrm{U} \mathrm{R} \mathrm{U}^{\prime} \mathrm{R}^{\prime}\right) 2 \mathrm{~F}^{\prime}$

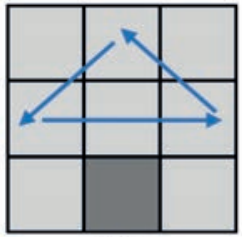

Ub

El lector puede comprobar la validez del esquema siguiente:

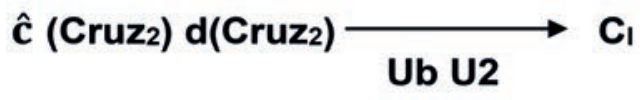

7.4.5 Dinámica del $\widehat{C}\left(\mathrm{Cruz}_{2}\right)$ en la capa superior
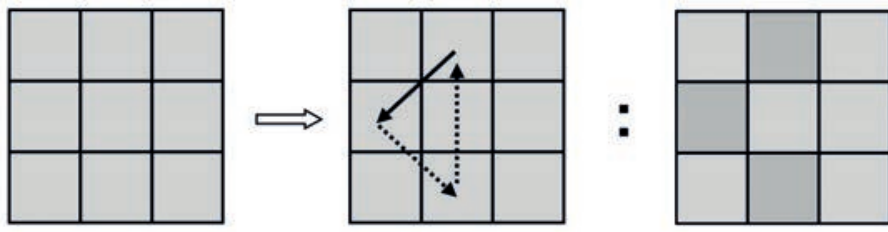

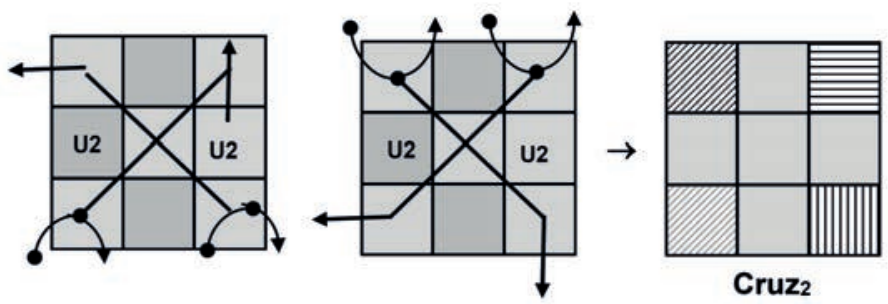

\subsubsection{Acción de $\hat{\mathbf{c}}\left(\mathrm{Cruz}_{2}\right)$ sobre sus aristas iniciales}

$\widehat{\mathrm{c}}\left(\mathrm{Cruz}_{2}\right)$ realiza una permutación en sentido antihorario de tres de las aristas distintas de la derecha (permanece invariante).

\subsubsection{Acción de $\hat{\mathbf{c}}\left(\mathrm{Cruz}_{2}\right)$ sobre sus vértices iniciales}

- Los vértices inferiores izquierdo y derecho reciben una torsión "horaria" de tres giros (1.6.3.3) y luego un movimiento U2.

- Los vértices superior, inferior y derecho reciben una torsión "antihoraria" de tres giros (1.6.3.3) y luego un movimiento U2.

\subsection{Nuevo constructor/deconstructor de $\mathrm{Cruz}_{2} \operatorname{con} \mathrm{mJ}_{1}$ y $\mathrm{T}_{2}$}

\subsubsection{Motivación:}

$\mathrm{Cruz}_{2}=\mathbf{m J}_{1}+\mathbf{T}_{2}$ Apliquemos el constructor canónico de $\mathrm{T}_{2}$ (según lo expuesto en 2.1.3) a la $\mathrm{mJ}_{1}$ de más abajo $\left(=\hat{\mathrm{c}}\left(\mathrm{mJ}_{\mathrm{I}}\right)\right)$
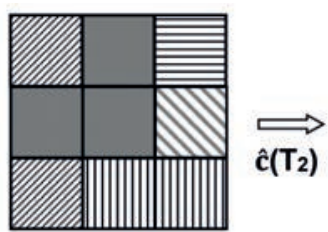

$\mathbf{m} \mathbf{J}_{1}$

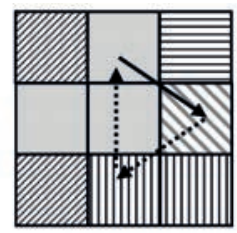

U
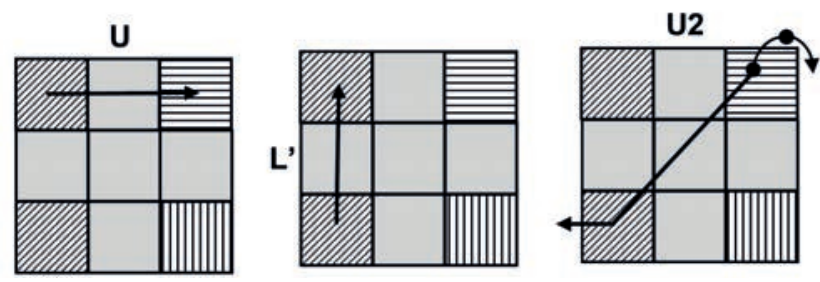


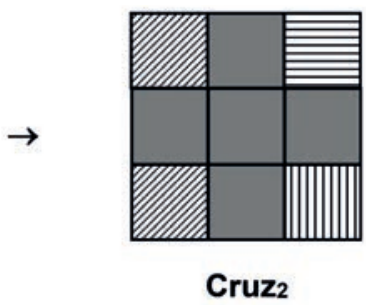

7.5.2 Nuevo constructor/deconstructor de la $\mathrm{Cruz}_{2}$

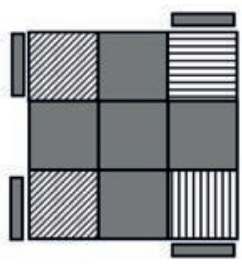

Cruz2 $1^{\text {a }}$ Forma: $\mathrm{Cruz}_{2}=m J_{1}+T_{2}$

$\mathbf{c}\left(\mathrm{Cruz}_{2}\right)=\hat{\mathbf{c}}\left(\mathbf{m} \mathbf{J}_{1}\right) \hat{\mathbf{c}}\left(\mathrm{T}_{2}\right)$

$\mathbf{d}\left(\mathrm{Cruz}_{2}\right)=\hat{d}\left(\mathrm{~T}_{2}\right) \hat{d}\left(\mathrm{~mJ}_{1}\right)$

$2^{a}$ Forma("canónica"):

$\hat{c}\left(\right.$ Cruz2 $\left._{2}\right)=R^{\prime}$ U2 R2 U R2 U R2 U2 R'

d̂(Cruz2) = R U2 R2 U' R2 U' R2 U2 R

(*) $\quad \hat{\mathbf{c}}\left(\mathrm{mJ}_{1}\right) \hat{\mathbf{c}}\left(\mathrm{T}_{2}\right) \quad=\mathbf{F}$ (U R U' R' )2 F' (F R' F' R URU' R') $\hat{\mathbf{d}}\left(\mathbf{T}_{2}\right) \hat{\mathbf{d}}\left(\mathbf{m J}_{1}\right)=($ RU R' U' R' F R F') $\quad$ F (R U R' U' $) 2$ F'

7.5.3 La no congruencia de las dos formas.

En la configuración de la $1^{\text {a }}$ forma todas las aristas quedan bien acomodadas (bien orientadas y bien posicionadas), lo que no sucede con la $2^{\mathrm{a}}$ forma.

7.5.4 La semejanza de la configuración de las dos formas: Intercambiando constructor/deconstructor.

7.5.4.1 Situación 1. p: $\mathbf{c}\left(\mathrm{Cruz}_{2}\right) \hat{\mathbf{d}}\left(\mathrm{Cruz}_{2}\right)$, donde $\mathrm{c}\left(\mathrm{Cruz}_{2}\right)=\mathrm{c}\left(\mathrm{mJ}_{1}\right) \mathrm{c}\left(\mathrm{T}_{2}\right)=\mathrm{F}\left(\mathrm{U}^{\mathrm{R}} \mathrm{U}^{\prime} \mathrm{R}^{\prime}\right) 2 \mathrm{~F}^{\prime}\left(\mathrm{F} \mathrm{R}^{\prime} \mathrm{F}^{\prime} \mathrm{R}\right.$ URU' R') $\widehat{\mathrm{d}}\left(\mathrm{Cruz}_{2}\right)=\mathrm{R}$ U2 R2 U' R2 U' R2 U2 R

El lector puede comprobar la validez del esquema siguiente: 


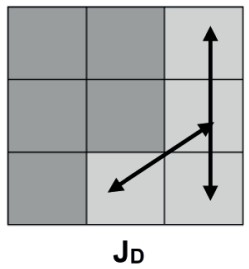

7.5.4.2 Situación 2. q: $\hat{\mathbf{c}}\left(\mathrm{Cruz}_{2}\right) \mathbf{d}\left(\mathrm{Cruz}_{2}\right)$, donde $\hat{\mathrm{c}}\left(\mathrm{Cruz}_{2}\right)=$ R' U2 R2 U R2 U R2 U2 R' $\mathrm{d}\left(\mathrm{Cruz}_{2}\right)=\mathrm{d}\left(\mathrm{T}_{2}\right) \mathrm{d}\left(\mathrm{mJ}_{1}\right)=\left(\right.$ RU R' U' R' F R F') $\mathrm{F}\left(\mathrm{R} \mathrm{U}^{\prime} \mathrm{U}^{\prime}\right)^{\prime} 2 \mathrm{~F}^{\prime}$ El lector puede comprobar la validez del esquema siguiente:

\section{$\hat{c}($ Cruzz) d(Cruzz)}

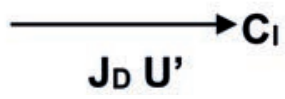

7.6 El Camaleón: $\mathrm{T}_{2}^{\mathrm{s}}+\mathrm{mL}_{1}$

\subsubsection{Motivación:}

Camaleón $=\hat{\mathbf{c}}\left(\mathrm{T}_{2}{ }^{\mathrm{s}}\right) \hat{\mathbf{c}}\left(\mathrm{mL}_{1}\right)$. Apliquemos el constructor canónico de $\mathrm{mL}_{1}$ (según lo expuesto en 6.1.1) a la $\mathrm{T}_{2}^{\mathrm{S}}$ de más abajo $\left(=\widehat{\mathrm{c}}\left(\mathrm{T}_{2}{ }^{\mathrm{S}}\right), 2.3 .1\right)$

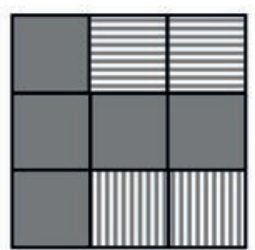

$\mathrm{T}_{2} \mathrm{~S}$
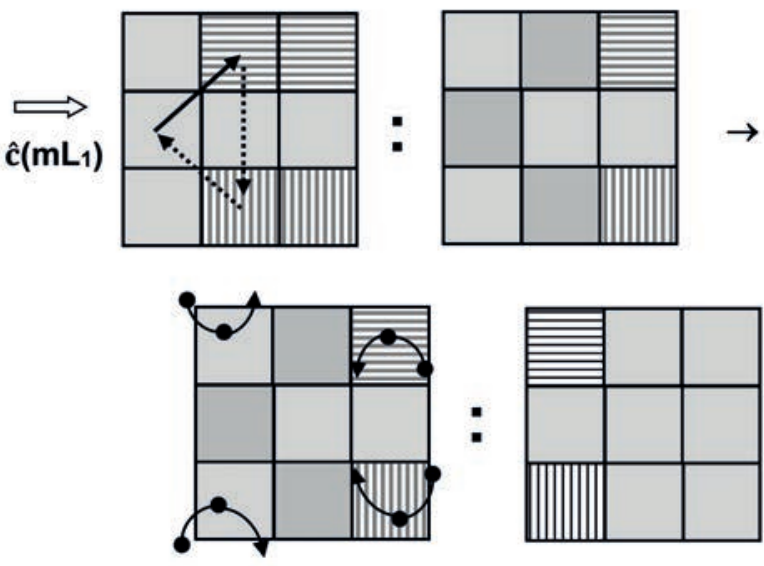
7.6.2 Dos formas de construir/deconstruir el Camaleón

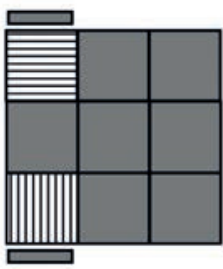

Camaleón

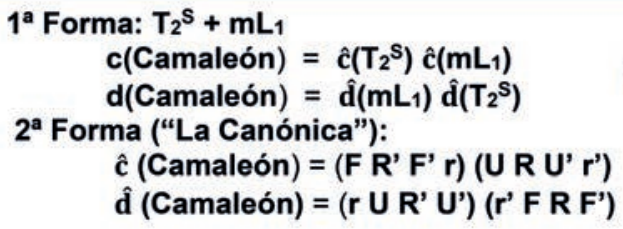

$\mathbf{r}=\mathbf{R M :}$ movimiento parecido al movimiento $\mathrm{R}$ solo que con " $r$ " se rotan simultáneamente las capas $R$ y su intermedia contigua $\mathrm{M}$ (sentido horario $90^{\circ}$ )

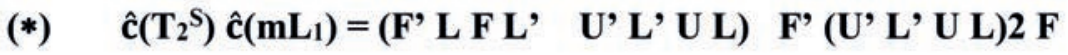
$\hat{d}\left(m_{1}\right) \hat{d}\left(T_{2}{ }^{S}\right)=F^{\prime}\left(L^{\prime} U^{\prime} L U^{\prime}\right) 2$ F (L' U' L U L F' L' F)

\subsubsection{La no congruencia de las dos formas}

En la configuración de la $2^{\text {a }}$ forma las aristas y el vértice inferior derecho quedan bien acomodados (bien posicionados y bien orientados); en tanto que la con la primera, si bien las aristas quedan bien acomodadas, el vértice inferior derecho no queda bien acomodado.

7.6.4 La semejanza en la configuración de las dos formas: Intercambiando constructor/deconstructor.

7.6.4.1 Situación 1.p: c(Camaleón) $\mathbf{d}$ (Camaleón), donde

$\mathrm{c}($ Camaleón $)=\hat{\mathrm{c}}\left(\mathrm{T}_{2}^{\mathrm{S}}\right) \hat{\mathrm{c}}\left(\mathrm{mL}_{1}\right)=\left(\mathrm{F}^{\prime} \mathrm{L} F\right.$ L' U' L' U L ) F' (U' L' U L)2 F $\widehat{\mathrm{d}}($ Camaleón $)=(\mathrm{r}$ U R' U') (r' F R F')

El lector puede comprobar la validez del esquema siguiente:

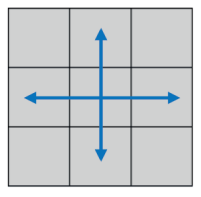

H
c(Camaleón) d̂ (Camaleón)

\section{H U2}

donde $\mathbf{H}=(\mathbf{M} 2 \mathbf{U} \mathbf{M} 2) \mathbf{U} 2$ (M2 U M2), 2.7.2 
7.6.4.2 Situación 2. q: $\hat{\mathbf{c}}$ (Camaleón) d(Camaleón), donde $\hat{c}($ Camaleón $)=\left(F^{\prime}\right.$ ' F' r) (U R U' r')

$\mathrm{d}($ Camaleón $)=\widehat{\mathrm{d}}\left(\mathrm{mL}_{1}\right) \hat{\mathrm{d}}\left(\mathrm{T}_{2}{ }^{\mathrm{S}}\right)=\mathrm{F}^{\prime}$ (L' U' L U)2 F (L' U' L U L F' L' F)

El lector puede comprobar la validez del esquema siguiente:

\section{$\hat{c}$ (Camaleón) d(Camaleón) $\longrightarrow C_{1}$ \\ H U2}

Comentario: El constructor canónico del Camaleón es muy parecido al de la $\mathrm{T}_{2}$, $c\left(T_{2}\right)=\left(F R^{\prime} F^{\prime} R\right)\left(U R U^{\prime} R^{\prime}\right)$. Solo se cambian las $R$ finales del primer y segundo párrafo por $\mathrm{r}$.

\subsubsection{Dinámica del $\widehat{c}$ (Camaleón) en la capa superior}

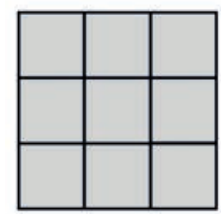

U

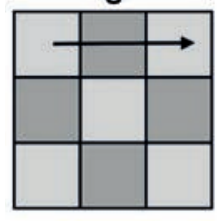

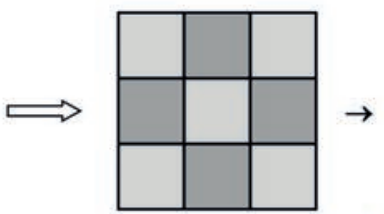

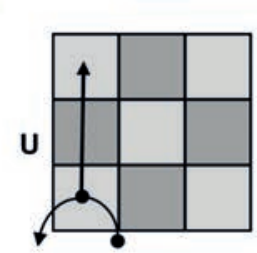

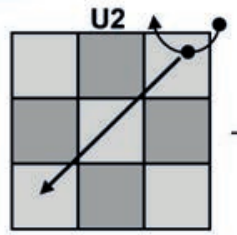

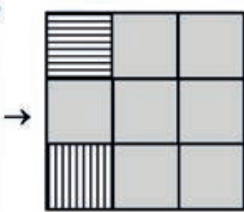

Camaleón

7.6.5.1 Acción de $\hat{\mathbf{c}}$ (Camaleón) sobre sus aristas iniciales $\widehat{\mathbf{c}}$ (Camaleón) deja invariantes las aristas: bien acomodadas (bien posicionadas y bien orientadas)

\subsubsection{Acción de $\hat{\mathbf{c}}$ (Camaleón) sobre sus vértices iniciales}

El vértice inferior derecho inicial queda invariante. Los otros tres giran en sentido horario pero los vértices de la diagonal principal exhiben torsión de tres giros, de modo preciso: 
- El vértice superior derecho inicial adquiere torsión horaria de tres giros y luego realiza un movimiento U2.

- El vértice inferior izquierdo inicial adquiere torsión antihoraria de tres giros y luego realiza un movimiento U.

Actividad para el lector: Una nueva forma para el Camaleón congruente a la canónica usando las configuraciones "T". Sean

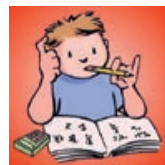

$$
\begin{aligned}
& c(\text { Camaleón })=\mathrm{U} 2 \mathrm{c}\left(\mathrm{T}_{2}\right) \mathrm{d}\left(\mathrm{T}_{1}\right) \mathrm{U} 2 \\
& \mathrm{~d}(\text { Camaleón })=\mathrm{U} 2 \mathrm{c}\left(\mathrm{T}_{1}\right) \mathrm{d}\left(\mathrm{T}_{2}\right) \mathrm{U} 2
\end{aligned}
$$

Usando el cubo de Rubik en su estado original comprobar que:

\section{c(Camaleón) $\mathbf{d}$ (Camaleón $)=$ Cubo en su estado original; y}

$\hat{\mathbf{c}}($ Camaleón $) \mathbf{c}($ Camaleón $)=$ Cubo en su estado original

\subsection{La Botella: Camaleón + Ab}

\subsubsection{Motivación:}

$\hat{\mathbf{c}}$ (Camaleón) Ab = Botella Apliquemos el PLL Ab a la configuración Camaleón de más abajo (= $\widehat{\mathbf{C}}$ (Camaleón), 6.6.1)

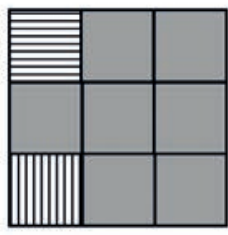

Camaleón
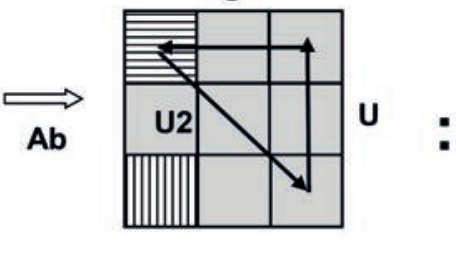

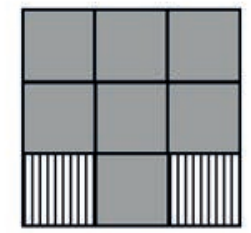

Botella

7.7.2 Dos formas congruentes de construir/deconstruir la Botella

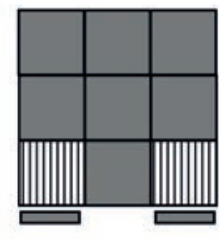

Botella
19 Forma: Botella $=$ Camaleón $+\mathbf{A b}$ $\mathbf{c}($ Botella $)=\hat{c}($ Camaleón $) \mathbf{A b}$ $\mathbf{d}($ Botella $)=$ Aa $\hat{\mathbf{d}}($ Camaleón $)$ (*)

$2^{\mathrm{a}}$ Forma ("La Canónica" $\cong 1^{\mathrm{a}}$ Forma): c (Botella): R U2 R D R' U2 R D' R2 d (Botella): R2 DR' U2 RD' R' U2 R' 
(*) $\hat{c}$ (Camaleón) Ab = (FR' F' R) M (UR U' R') M' R2 B2 R2 R' F R' B2 R F' R Aa đ(Camaleón) = R' FR' B2 R F' R R2 B2 R2 M (RUR' U') M' (R' FR F')

Comentario: Ambas formas son congruentes. El lector usando el cubo de Rubik en su estado original puede comprobar que:

$$
\begin{aligned}
& c(\text { Botella }) \hat{d}(\text { Botella })=\text { Cubo en su estado original; } y \\
& \hat{c}(\text { Botella }) c(\text { Botella })=\text { Cubo en su estado original. }
\end{aligned}
$$

7.7.3 Dinámica del $\widehat{c}$ (Botella) en la capa superior
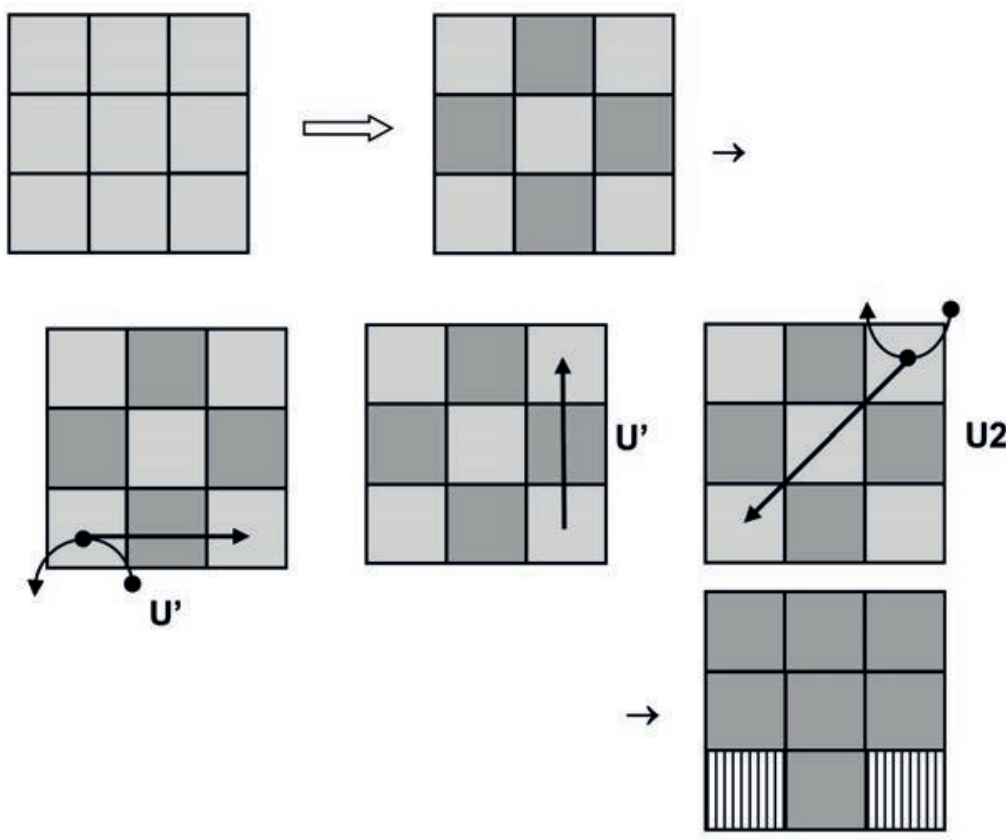

Botella

\subsubsection{Acción de $\hat{\mathbf{c}}$ (Botella) sobre sus aristas iniciales}

$\widehat{\mathbf{c}}$ (Botella) deja invariantes las aristas: bien acomodadas (bien posicionadas y bien orientadas)

\subsubsection{Acción de $\hat{\mathbf{c}}$ (Botella) sobre sus vértices iniciales}

El vértice superior izquierdo inicial queda invariante. Los otros tres giran en sentido antihorario, pero los vértices de la diagonal principal exhiben torsión de tres giros, de modo preciso: 
- El vértice superior derecho inicial adquiere torsión horaria de tres giros y luego realiza un movimiento $\mathrm{U} 2$.

- El vértice inferior izquierdo inicial adquiere torsión antihoraria de tres giros y luego realiza un movimiento U'.

7.8 Constructor/deconstructor de la Botella usando las " $T$ "

Botella $=\mathrm{T}_{1}^{-\mathrm{S}}+\mathrm{U}^{\prime}+\mathrm{T}_{2}$.

\subsubsection{Motivación:}

Botella $=\hat{\mathbf{d}}\left(\mathrm{T}_{1}{ }^{\mathrm{S}}\right) \mathbf{U}^{\prime} \hat{\mathbf{c}}\left(\mathrm{T}_{2}\right)$. Se Aplica un movimiento $\mathrm{U}^{\prime} \mathrm{y}$ luego el constructor canónico de $\mathrm{T}_{2}$ (según lo expuesto en 2.1.3) a la $\mathrm{q}_{1}\left(=\mathrm{d}\left(\mathrm{T}_{1}{ }^{\mathrm{S}}\right), 1.9 .1\right)$

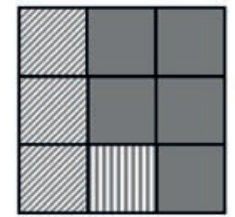

$\mathbf{q}_{1}$
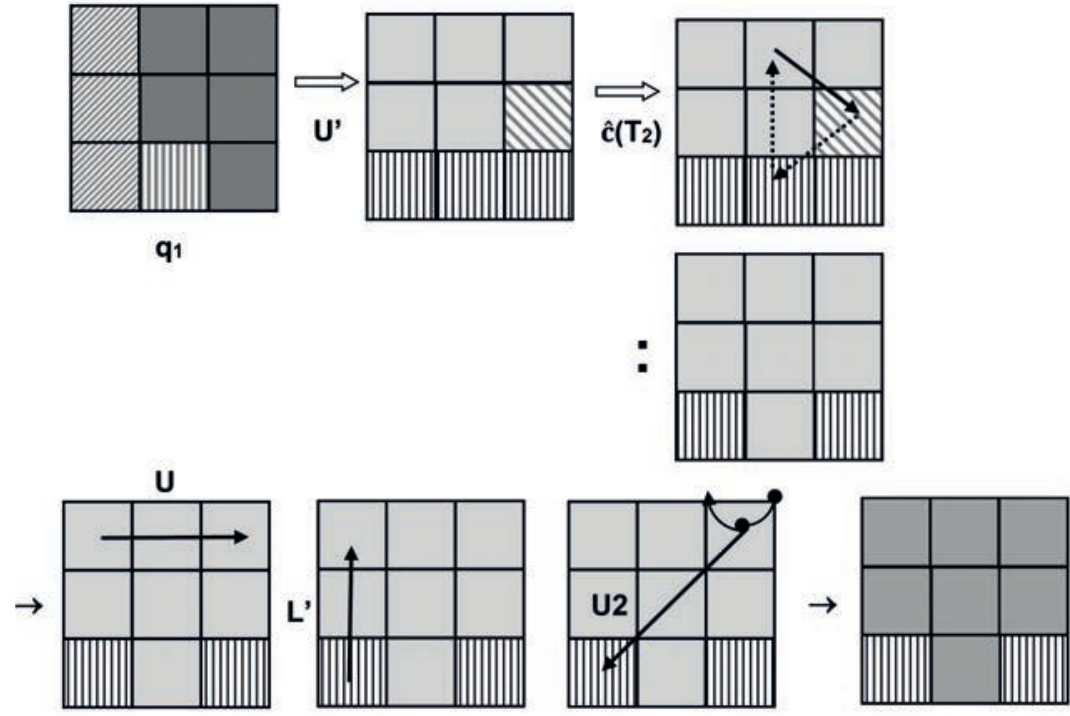

\subsubsection{Nuevos constructor/deconstructor de la Botella}

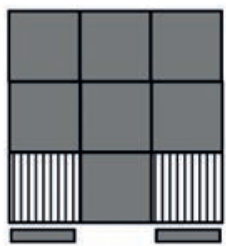

$1^{\text {a }}$ Forma (Nueva): Botella $=T_{1}^{-S}+U^{\prime}+T_{2}$ $\mathbf{c}($ Botella $)=\hat{d}\left(T_{1}{ }^{S}\right) U^{\prime} \hat{c}\left(T_{2}\right)$ $\mathbf{d}($ Botella $)=\hat{d}\left(T_{2}\right) U \hat{c}\left(T_{1} s\right)$

$2^{\mathrm{a}}$ Forma ("La Canónica"):

$\hat{c}$ (Botella) = R U2 R D R' U2 R D' R2 d̂ (Botella) = R2 DR' U2 RD' R' U2 R'

\section{Botella}


(*) $\hat{\mathbf{d}}\left(\mathbf{T}_{1}^{\mathrm{S}}\right) \mathbf{U}^{\prime} \hat{\mathbf{c}}\left(\mathbf{T}_{2}\right)$ = (F' L' U' L U F) U' (F R' F' R U R U' R') $\hat{\mathbf{d}}\left(\mathbf{T}_{2}\right) \mathbf{U} \hat{\mathbf{c}}\left(\mathbf{T}_{1}{ }^{\mathrm{S}}\right)=($ R U R' U' R' F R F') U (F' U' L' U L F)

7.8.3 La no congruencia de las dos formas.

En la configuración de la $2^{\text {a }}$ forma todas las aristas quedan bien acomodados (bien posicionadas y bien orientadas); lo que no sucede con la $1^{\text {a }}$ forma.

7.8.4 La semejanza en la configuración de las dos formas: Intercambiando constructor/deconstructor.

7.8.4.1 Situación 1. p: c(Botella) $\mathbf{d}$ (Botella), donde $c($ Botella $)=\hat{d}\left(T_{1}{ }^{S}\right) U^{\prime} \hat{c}\left(T_{2}\right)=\left(F^{\prime} L^{\prime} U^{\prime} L\right.$ U F) U' (F R' F' R U R U' R') $\hat{\mathrm{d}}($ Botella $)=$ R2 DR' U2 RD' R' U2 R'

El lector puede comprobar la validez del esquema siguiente:

$$
\text { c(Botella) } \hat{\mathbf{d}} \text { (Botella) }
$$

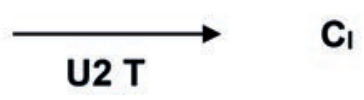

donde T = RU R' U' R' FR2 U'R' U’ RU R'F', 6.4.2 (Capítulo 6)

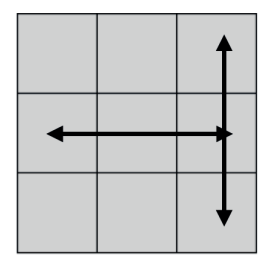

$\mathbf{T}$

7.8.4.2 Situación 2. q: ĉ (Botella) d(Botella), donde $\hat{\mathrm{c}}($ Botella) $=$ R U2 R D R' U2 R D' R2 $\mathrm{d}($ Botella $)=\hat{\mathrm{d}}\left(\mathrm{T}_{2}\right) \mathrm{U} \hat{\mathrm{c}}\left(\mathrm{T}_{1}^{\mathrm{S}}\right)=($ RU R' U' R' F R F') U (F' U' L' U L F)

El lector puede comprobar la validez del esquema siguiente: 


\section{ĉ (Botella) d(Botella)}

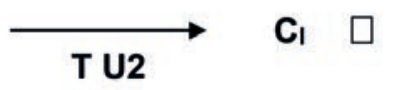

7.9 La Pajarita: Camaleón + Aa Inverso canónico del Camaleón

\subsubsection{Motivación:}

ĉ (Camaleón) Aa = Pajarita Apliquemos el PLL Aa a la configuración Camaleón de más abajo (= ĉc(Camaleón) , 6.6.1)

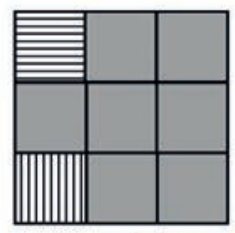

Camaleón

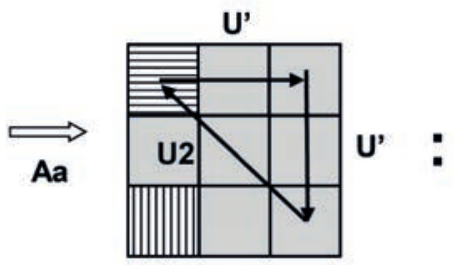

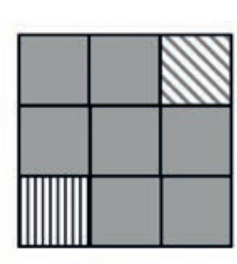

Pajarita

\subsubsection{Dos formas de construir/deconstruir la Pajarita}

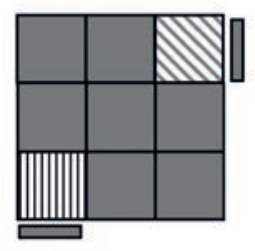

Pajarita

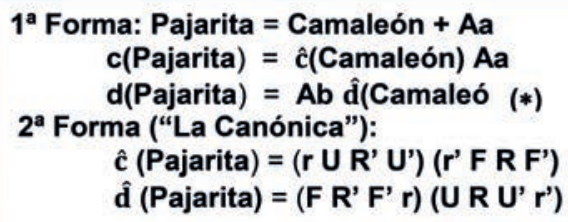

$\mathbf{r}=\mathbf{R M}$ : movimiento parecido al movimiento $\mathrm{R}$ solo que con " $r$ " se rotan simultáneamente las capas $R$ y su intermedia contigua $\mathrm{M}$ (sentido horario $90^{\circ}$ )

(*) $\hat{c}$ (Camaleón) $\mathrm{Aa}=\left(\mathrm{FR}^{\prime} \mathrm{F}^{\prime} \mathrm{R}\right) \mathrm{M}$ (UR U' R') M' R' FR' B2 R F' R R2 B2 R2 $\mathrm{Ab}$ d(Camaleón) = R2 B2 R2 R' F R' B2 R F' R M (RUR' U' ) M' (R' FR F')

\subsubsection{La no congruencia de las dos formas}

En la configuración de la $2^{\text {a }}$ forma todas las aristas quedan bien acomodados (bien posicionadas y bien orientadas); lo que no sucede con la $1^{\text {a }}$ forma.

7.9.4 La semejanza de la configuración de las dos formas. Intercambiando constructor/deconstructor. 
7.9.4.1 Situación 1. p: c(Pajarita) $\mathbf{d}$ (Pajarita), donde

$\mathrm{c}($ Pajarita $)=\widehat{\mathbf{c}}($ Camaleón $)$ Aa

$=(F R$ 'F' R) M (UR U' R') M' R'FR' B2 R F' R' R2 B2 R2

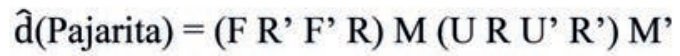

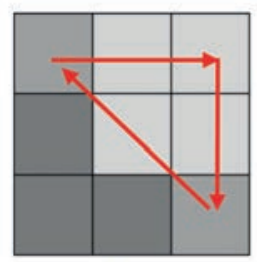

Aa

El lector puede comprobar la validez del esquema siguiente:

c(Pajarita) $\hat{\mathbf{d}}$ (Pajarita)

$\mathrm{Aa}$

$\mathbf{C}_{\mathbf{l}}$

7.9.4.2 Situación 2. q: $\hat{\mathbf{c}}$ (Pajarita) d(Pajarita), donde

$\hat{\mathrm{c}}($ Pajarita $)=\mathrm{M}(\mathrm{R}$ U R' U') M' (R' F R F')

$\mathrm{d}($ Pajarita $)=\mathrm{Ab} \widehat{\mathrm{d}}($ Camaleón $)$

= R2 B2 R2 R'FR' B2RF'R M (RUR' U') M' (R' FR F')

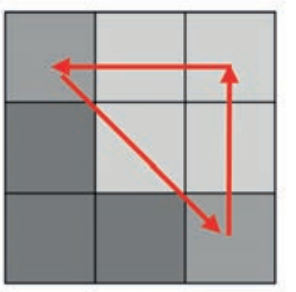

$\mathbf{A b}$

El lector puede comprobar la validez del esquema siguiente:

$\hat{\mathbf{c}}$ (Pajarita) d(Pajarita)

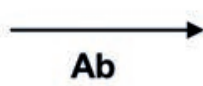

$\mathbf{C}_{\mathbf{l}}$

Comentario: El constructor canónico de la Pajarita es muy parecido al deconstructor de la $T_{2}, d\left(T_{2}\right)=\left(R \cup R^{\prime} U^{\prime}\right)\left(R^{\prime} F R F^{\prime}\right)$. Solo se cambian las $R$ iniciales del primer y segundo párrafo por $r$. 


\subsubsection{Dinámica del $\widehat{c}$ (Pajarita) en la capa superior}
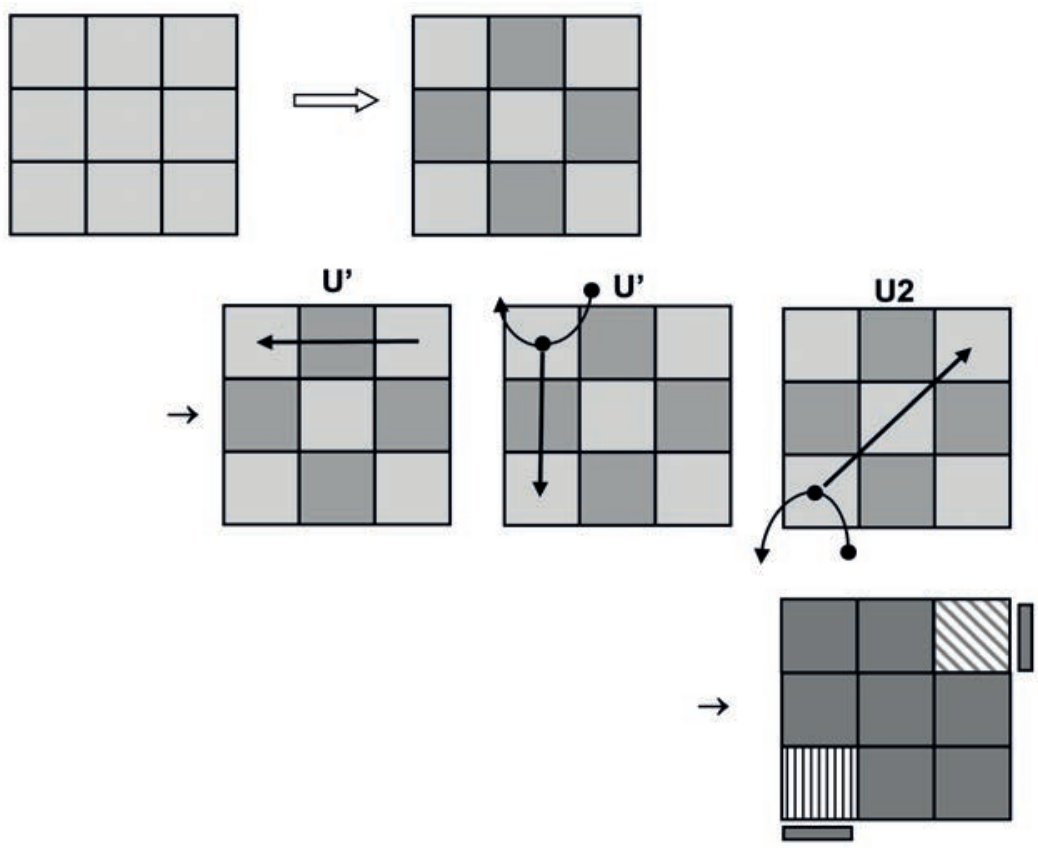

Pajarita

7.9.5.1 Acción de $\hat{\mathbf{c}}$ (Pajarita) sobre sus aristas iniciales $\hat{\mathrm{c}}$ (Pajarita) deja invariantes las aristas: bien acomodadas (bien posicionadas y bien orientadas)

\subsubsection{Acción de $\hat{\mathbf{c}}$ (Pajarita) sobre sus vértices iniciales}

El vértice inferior derecho inicial queda invariante. Los otros tres giran en sentido antihorario, pero los vértices izquierdos iniciales exhiben torsión de tres giros, de modo preciso:

- El vértice inferior izquierdo inicial adquiere torsión antihoraria de tres giros y luego realiza un movimiento U2.

- El vértice superior izquierdo inicial adquiere torsión horaria de tres giros y luego realiza un movimiento U'. 
7.10 Constructor/deconstructor de la Pajarita usando las “ $T$ ”

\subsubsection{Motivación:}

Pajarita $=\hat{\mathbf{c}}\left(\mathrm{T}_{1}\right) \hat{\mathbf{d}}\left(\mathrm{T}_{2}\right)$ U2 Apliquemos el deconstructor canónico de $\mathrm{T}_{2}\left(=\right.$ Cometa $\left._{1}\right)$ a $\mathrm{T}_{1}$, según lo visto en 2.2.2, $\mathrm{y}$ luego a la configuración así obtenida démosle una rotación de $180^{\circ}$ en la capa superior (U2).
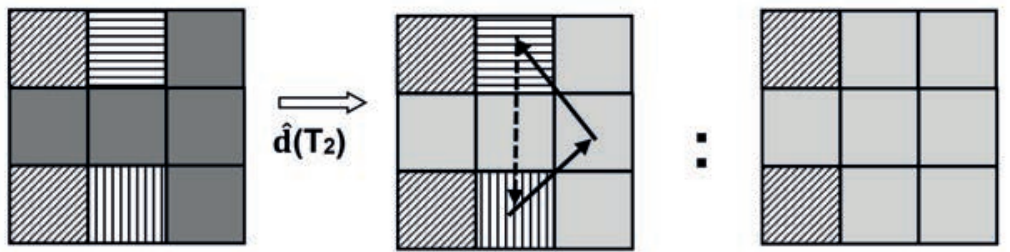

$T_{1}$

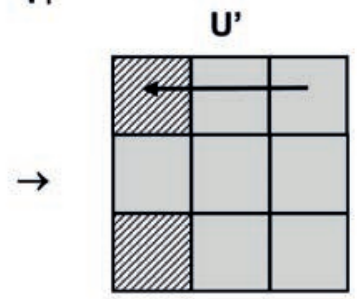

$\mathbf{L}$
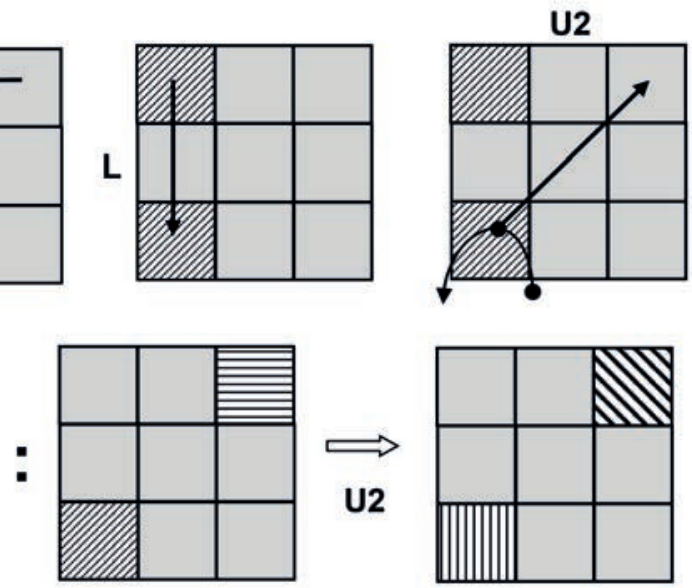

7.10.2 Nuevo constructor/deconstructor de la Pajarita
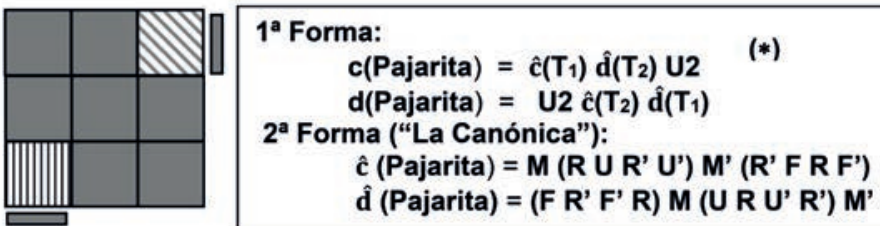

Pajarita

(*) $\quad \hat{\mathbf{c}}\left(\mathbf{T}_{1}\right) \hat{\mathbf{d}}\left(\mathrm{T}_{2}\right) \mathbf{U} \mathbf{2}=($ F U R U' R' F') (R U R' U' R' F R F') U2

U2 $\hat{c}\left(T_{2}\right) \hat{d}\left(T_{1}\right)=$ U2 (F R' F' R U R U' R') (F R U R' U' F') 
7.10.3 La no congruencia de las dos formas

En la configuración de la $2^{a}$ forma todas las aristas y el vértice inferior derecho quedan bien acomodados (bien posicionados y bien orientados); lo que no sucede con la $1^{\text {a }}$ forma.

7.10.4 La semejanza de la configuración de las dos formas Intercambiando constructor/deconstructor.

7.10.4.1 Situación 1. p: c(Pajarita) d̂ (Pajarita), donde $\mathrm{c}($ Pajarita $)=\hat{\mathrm{c}}\left(\mathrm{T}_{1}\right) \hat{\mathrm{d}}\left(\mathrm{T}_{2}\right) \mathrm{U} 2=\left(\mathrm{FUR} \mathrm{U}^{\prime} \mathrm{R}^{\prime} \mathrm{F}^{\prime}\right)\left(\mathrm{R}^{\mathrm{U}} \mathrm{R} \mathrm{R}^{\prime} \mathrm{U}^{\prime} \mathrm{R}^{\prime} \mathrm{F}\right.$ R F') U2 $\widehat{\mathrm{d}}($ Pajarita $)=\left(F^{\prime} \mathrm{R}^{\prime} \mathrm{F}^{\prime} \mathrm{R}\right) \mathrm{M}$ (U R U' R') M'

El lector puede comprobar la validez del esquema siguiente:

$$
\text { c(Pajarita) } \mathbf{d}(\text { Pajarita) }
$$

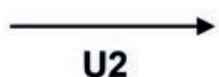

$\mathbf{C}_{1}$

U2

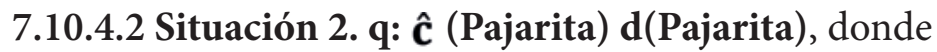

$$
\hat{c} \text { (Pajarita) }=M(R \text { U R' U') M' (R' F R F') }
$$

$\mathrm{d}\left(\right.$ Pajarita) $=\mathrm{U} 2 \hat{\mathrm{c}}\left(\mathrm{T}_{2}\right) \hat{\mathrm{d}}\left(\mathrm{T}_{1}\right)=\mathrm{U} 2$ (FR' F' R U R U' R') (FRU R' U' F')

El lector puede comprobar la validez del esquema siguiente:

ĉ (Pajarita) d(Pajarita)

$\mathbf{C}_{1}$

U2 
Síntesis tabular OLL “Canónica” (Capítulo 7)

5 OLL para practicar y memorizar

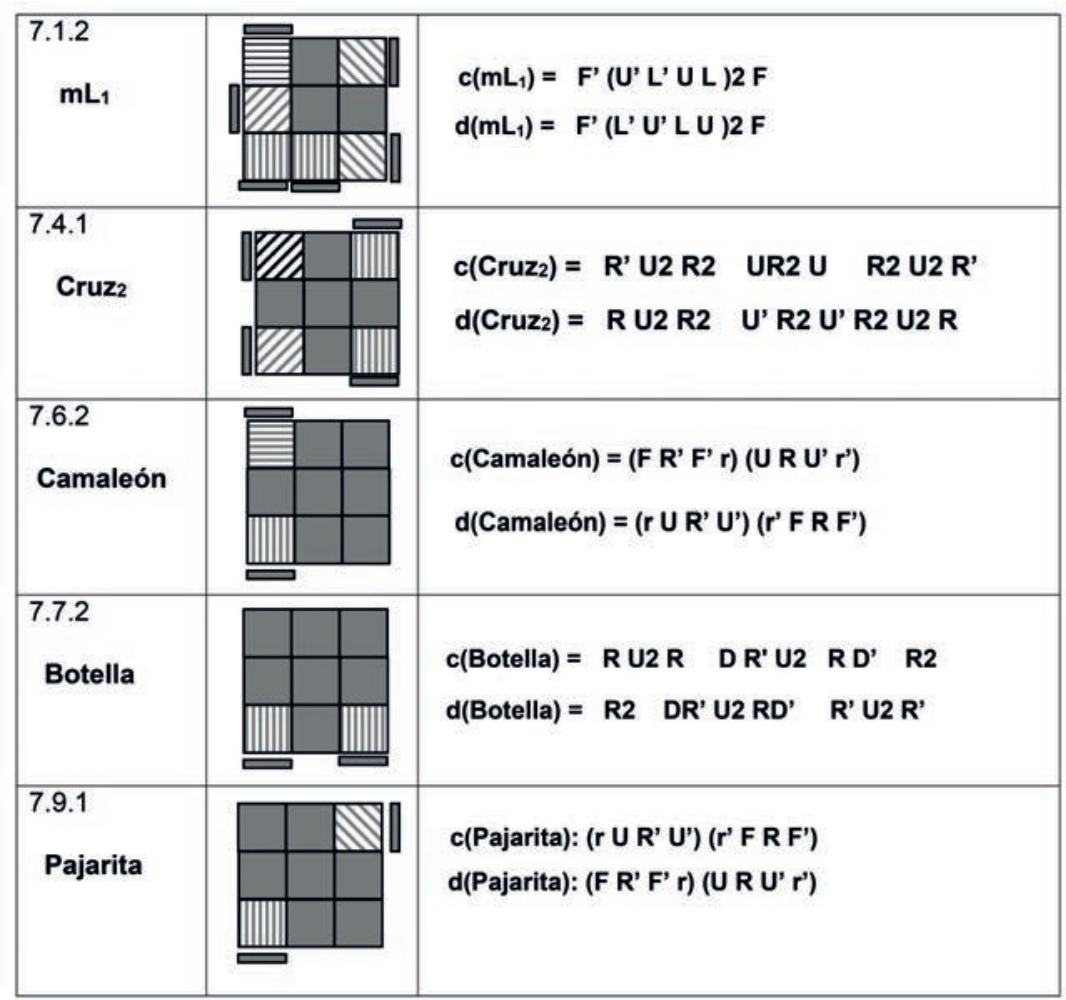




\section{Los dos Peces Básicos Completando el Grupo de "Las 7 cruces ampliadas"}

En los capítulos 4 y 7 hemos conocido cinco configuraciones OLL de "Cruz Ampliada": La Cruz (4.7.2); la Cruz, el Camaleón, la Botella y la Pajarita (7.4.1, 7.6.1, 7.7.2 y 7.9.1 respectivamente). Bien, en este trataremos otras dos, como son los peces básicos $\mathrm{Pez}_{\mathrm{R}}$ y $\mathrm{Pez}_{\mathrm{L}}$, que completan las 7 clásicas "cruces ampliadas". El nombre de "cruces ampliadas" decíamos obedece al hecho de que la propiedad fundamental de los elementos de ese grupo es que todos sus elementos tienen sus cuatro aristas bien orientadas.

Observaremos formas constructivas de los elementos de este grupo a partir de tales peces y de ahí que se les denominen "Básicos". Y volveremos a usar una vez más la adición de configuraciones:

$$
\mathbf{A}=\mathbf{B}+\mathbf{U k}+\mathbf{C}+\mathbf{U m} \text { significa que } \hat{\boldsymbol{c}}(\mathbf{A})=\hat{\boldsymbol{c}}(\mathbf{A}) \mathbf{U k} \hat{\boldsymbol{c}}(\mathbf{B}) \mathbf{U m}
$$

\subsection{El Primer Pez Básico: $\mathrm{Pez}_{\mathrm{R}}$.}

\subsubsection{Motivación:}

$\mathrm{Pez}_{\mathrm{R}}=\mathrm{mL}_{1}+\mathrm{U}^{\prime}+\mathrm{T}_{1}$

Construimos $\mathrm{mL}_{1}$, luego damos un giro U' y por último a esta configuración aplicamos el constructor canónico de $\mathrm{T}_{1}$ (según lo expuesto en 1.6.3).

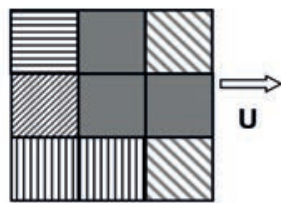

$\mathrm{mL}_{1}$

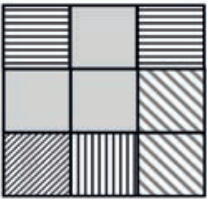

U

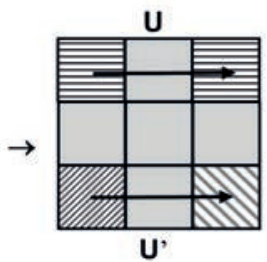

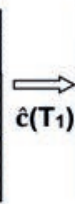
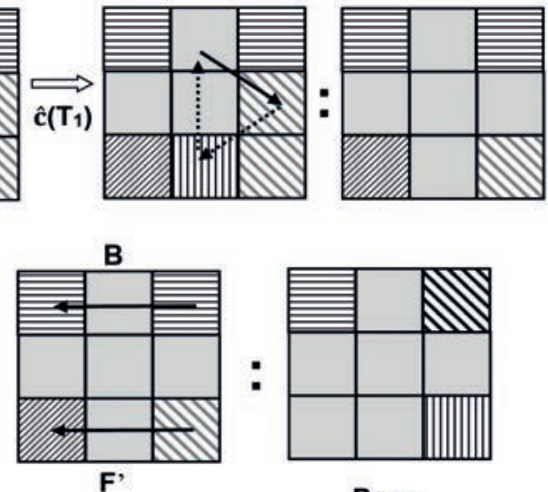

PezR 
8.1.2 Dos Formas de construir/deconstruir PezR

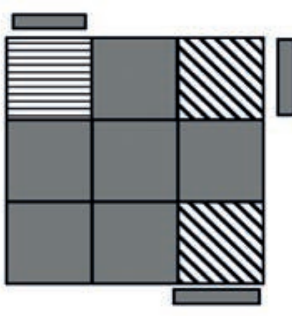

PezR 1a Forma: $P^{\text {a }} Z_{R}=m L_{1}+U^{\prime}+T_{1}$

$$
\begin{aligned}
& c\left(P_{e}\right)=c\left(m_{1}\right) U^{\prime} c\left(T_{1}\right) \\
& d\left(P_{R}\right)=d\left(T_{1}\right) U d\left(m L_{1}\right)
\end{aligned}
$$

2a Forma ("canónica"):

$$
\begin{aligned}
& \hat{c} \text { (PezR) = R U2 R' U' R U' R' } \\
& \widehat{d}\left(\text { PezR }^{\prime}=\mathbf{R} \cup \mathbf{R}^{\prime} \mathbf{R}\right. \text { U2 R' }
\end{aligned}
$$

$$
\begin{aligned}
& c\left(m_{1}\right) U^{\prime} c\left(T_{1}\right)=F^{\prime}\left(U^{\prime} L^{\prime} U \text { L }\right) 2 \text { F U' F URU'R' F' } \\
& \mathrm{d}\left(\mathrm{T}_{1}\right) \mathrm{U} \mathbf{d}\left(\mathrm{mL}_{1}\right)=\text { F RUR'U' F' U F' (L' U' L U)2 F }
\end{aligned}
$$

8.1.3 La no congruencia de las dos formas

En la configuración de la $1^{\text {a }}$ forma el vértice inferior izquierdo inicial de la capa superior queda bien acomodado (bien posicionado y bien orientado), lo que no sucede con la $2^{\text {a }}$ forma.

\subsubsection{La semejanza en la configuración de las dos formas:}

\section{Intercambiando constructor/deconstructor}

8.1.4.1 Situación 1.p: $c\left(\operatorname{Pez}_{R}\right) \hat{\mathbf{d}}\left(\operatorname{Pez}_{R}\right)$, donde

$$
\begin{aligned}
& \mathrm{c}\left(\text { PezR }_{\mathrm{R}}\right)=\mathrm{c}\left(\mathrm{mL}_{1}\right) \mathrm{U}^{\prime} \mathrm{c}\left(\mathrm{T}_{1}\right)=\mathrm{F}^{\prime}\left(\mathrm{U}^{\prime} \mathrm{L}^{\prime} \mathrm{U} \mathrm{L}\right) 2 \mathrm{~F} \quad \mathrm{U}^{\prime} \text { F URU' F' } \\
& \hat{\mathrm{d}}\left(\mathrm{Pez}_{\mathrm{R}}\right)=\mathrm{R} \mathrm{U}^{\prime} \mathrm{U} \quad \text { R U2 R' }
\end{aligned}
$$

Diseñar la configuración $\mathrm{Pez}_{\mathrm{R}}$ a partir de la $1^{\mathrm{a}}$ forma (constructor $\mathrm{c}\left(\mathrm{Pez}_{\mathrm{R}}\right)$ y orientar el cubo con la $2^{\mathrm{a}}$ forma (deconstructor $\left.\hat{\mathrm{d}}\left(\mathrm{Pez}_{\mathrm{R}}\right)\right)$.

Resulta válido el siguiente diagrama:

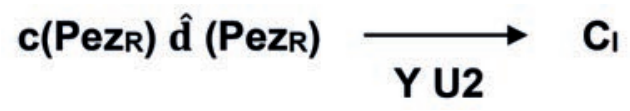

$$
\begin{aligned}
& \text { con } \left.Y \text { : F R (U' R' U') (R U R' F') d(T } T_{2}\right), 5.11 .3 \\
& \text { y } d\left(T_{2}\right)=\left(R \text { R }^{\prime} U^{\prime}\right) \text { (R' F R F'). }
\end{aligned}
$$


8.1.4.2 Situación 2. q: $\hat{\mathbf{c}}\left(\mathrm{Pez}_{\mathrm{p}}\right) \mathbf{d}\left(\mathrm{Pez}_{\mathrm{p}}\right)$, donde

$$
\begin{aligned}
& \hat{\mathrm{c}}\left(\operatorname{Pez}_{\mathrm{R}}\right)=\left(\mathrm{R} \mathrm{U}^{2} \mathrm{R}^{\prime} \mathrm{U} 2\right)\left(\mathrm{U}^{\mathrm{R}} \mathrm{U}^{\prime} \mathrm{R}^{\prime}\right) \\
& \mathrm{d}\left(\mathrm{Pez}_{\mathrm{R}}\right)=\mathrm{d}\left(\mathrm{T}_{1}\right) \mathrm{Ud}\left(\mathrm{mL}_{1}\right)=\mathrm{F} \text { URU' F' U F' (L' U' L U)2 F }
\end{aligned}
$$

Diseñar la configuración $\mathrm{Pez}_{\mathrm{R}}$ a partir de la $2^{\mathrm{a}}$ forma $\left(\hat{c}\left(P e z_{R}\right)\right)$ y orientar el cubo con la $1^{\text {a }}$ forma (deconstructor $\left.\mathrm{d}\left(\mathrm{Pez}_{\mathrm{R}}\right)\right)$.

Resulta válido el siguiente diagrama

$$
\hat{c}\left(P_{R}\right) d\left(P_{e Z R}\right) \underset{\text { U2 Y }}{\longrightarrow} C_{1}
$$

\subsubsection{Dinámica del $\hat{c}\left(\mathrm{Pez}_{\mathrm{R}}\right)$ en la capa superior}

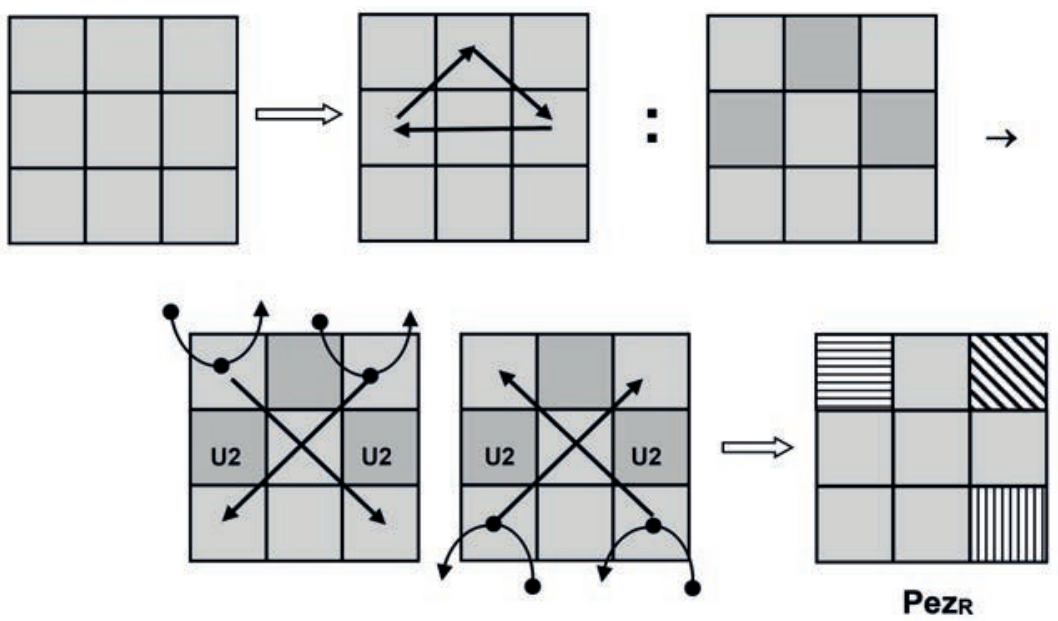

8.1.5.1 Acción de $\hat{\mathbf{c}}\left(\mathrm{Pez}_{\mathrm{R}}\right)$ sobre sus aristas iniciales $\hat{\mathbf{c}}$ $\left(\mathrm{Pez}_{\mathrm{R}}\right)$ realiza una permutación en sentido horario de tres de las aristas distintas de la inferior (permanece invariante).

8.1.5.2 Acción de $\hat{\mathbf{c}}\left(\mathrm{Pez}_{\mathrm{R}}\right)$ sobre sus vértices iniciales Todos los vértices rotan $180^{\circ}$ en la capa superior (U2) pero los distintos al superior derecho inicial reciben torsiones antihorarias de tres giros (1.6.3.3). 
8.2 El segundo Pez Básico: $\mathrm{Pez}_{\mathrm{L}}$ ó Simétrico de $\mathrm{Pez}_{\mathrm{R}}{ }^{27}$

\subsubsection{Motivación:}

$$
\operatorname{Pez}_{\mathrm{L}}=\mathrm{mJ}_{1}+\mathrm{U}+\mathrm{T}_{1}^{\mathrm{s}}
$$

Construimos $\mathrm{mJ}_{1}$, luego damos un giro $\mathrm{U}$ y por último a esta configuración aplicamos el constructor canónico de $\mathrm{T}_{1}^{\mathrm{S}}$ (según lo expuesto en 1.8.3).
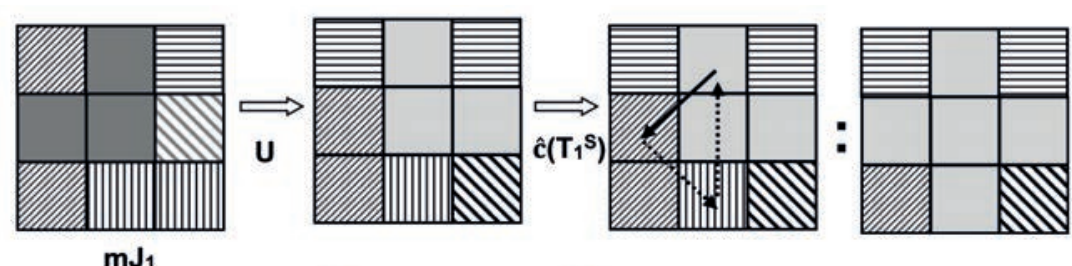

$\mathbf{m} \mathbf{J}_{1}$
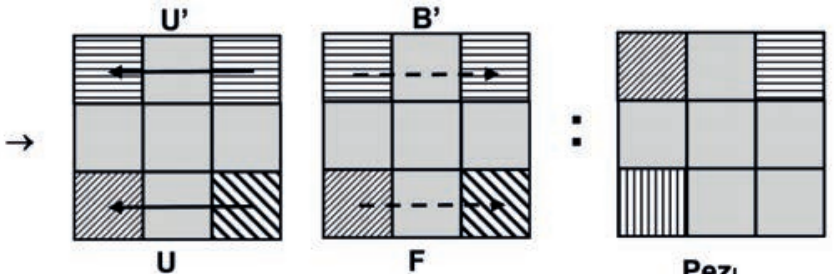

PezL

\subsubsection{Dos Formas de construir/deconstruir PezL}

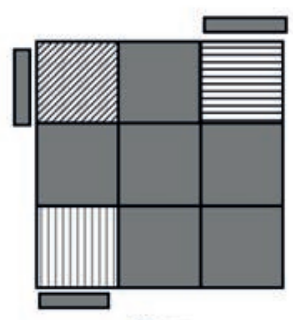

PezL

$$
\begin{aligned}
& \text { 1 }{ }^{\text {a Forma: }} \text { PezL }=\mathrm{mJ}_{1}+\mathrm{U}+\mathrm{T}_{1} \mathrm{~s} \\
& c(\text { Pezl })=c\left(m_{J_{1}}\right) \cup c\left(T_{1}\right)^{s} \\
& d(\text { PezL })=d\left(T_{1}\right)^{s} U^{\prime} d\left(m J_{1}\right)
\end{aligned}
$$

$2^{\text {a }}$ Forma ("canónica"):

$$
\begin{array}{ll}
\hat{c}(\text { PezL })=L^{\prime} U 2 L & \text { U L'U L } \\
\widehat{d}(\text { PezL })=L^{\prime} U^{\prime} L U^{\prime} & L^{\prime} U 2 L
\end{array}
$$

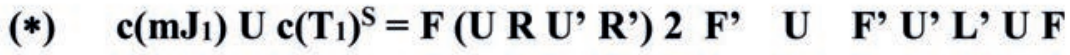
d(T $\left.\mathbf{T}_{1}\right)^{\mathrm{S}} \mathbf{U}^{\prime} \mathbf{d}\left(\mathrm{mJ}_{1}\right)=\mathrm{F}^{\prime} \mathbf{U}^{\prime} \mathrm{L}^{\prime}$ U F U' F (RU R' U')2 F' 


\subsubsection{La no congruencia de las dos formas}

En la configuración de la $1^{\text {a }}$ forma el vértice inferior derecho inicial de la capa superior queda bien acomodado (bien posicionado y bien orientado), lo que no sucede con la $2^{\text {a }}$ forma.

8.2.4 La semejanza en la configuración de las dos formas: Intercambiando constructor/deconstructor

8.2.4.1 Situación 1. p: $c\left(\operatorname{Pez}_{\mathrm{L}}\right) \hat{\mathbf{d}}\left(\mathrm{Pez}_{\mathrm{L}}\right)$, donde $\mathrm{c}\left(\mathrm{Pez}_{\mathrm{L}}\right)=\mathrm{c}\left(\mathrm{mJ}_{1}\right) \mathrm{U} \mathrm{c}\left(\mathrm{T}_{1}\right)^{\mathrm{S}}=\mathrm{F}\left(\mathrm{U}^{\mathrm{R}} \mathrm{U}^{\prime} \mathrm{R}^{\prime}\right) 2 \mathrm{~F}^{\prime} \quad \mathrm{U}^{\prime} \quad \mathrm{F}^{\prime} \mathrm{U}^{\prime} \mathrm{L}^{\prime} \mathrm{U} F$ $\widehat{\mathrm{d}}\left(\mathrm{Pez}_{\mathrm{L}}\right)=\mathrm{L}^{\prime} \mathrm{U}^{\prime} \mathrm{L} \mathrm{U}^{\prime} \quad$ L' U2 L
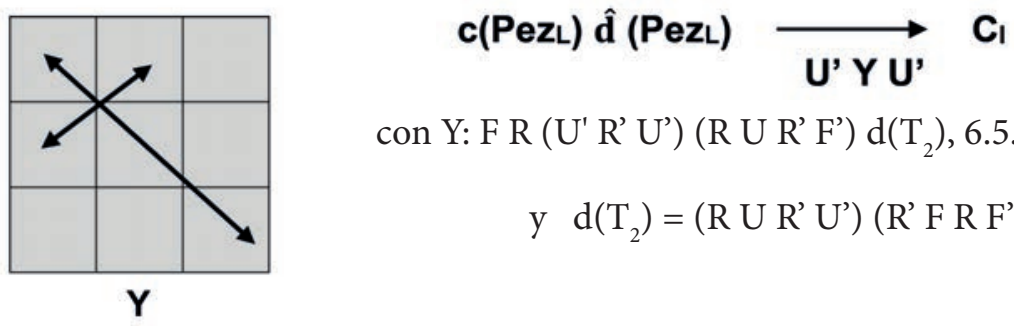

con Y: F R (U' R' U') (R U R' F') d( $\left.\mathrm{T}_{2}\right)$, 6.5.2 y $d\left(T_{2}\right)=($ R U R' U') (R' F R F').

8.2.4.2 Situación 2. q: $\hat{\mathbf{c}}\left(\mathrm{Pez}_{\mathrm{L}}\right) \mathrm{d}\left(\mathrm{Pez}_{\mathrm{L}}\right)$, donde

$$
\begin{aligned}
& \hat{\mathrm{c}}\left(\mathrm{PeZ}_{\mathrm{L}}\right)=\mathrm{L}^{\prime} \mathrm{U} 2 \mathrm{~L} \quad \mathrm{U} \mathrm{L}^{\prime} \mathrm{U} \mathrm{L} \\
& d\left(P e z_{L}\right)=d\left(T_{1}\right)^{S} U^{\prime} d\left(m_{1}\right)=F^{\prime} U^{\prime} L^{\prime} U_{F} \quad U^{\prime} \quad F\left(R R^{\prime} U^{\prime}\right) 2 F^{\prime} \\
& \hat{c} \text { (PezL) d(PezL) } \underset{U Y U}{\longrightarrow} C_{I}
\end{aligned}
$$

8.2.5 Dinámica del $\widehat{C}\left(\mathrm{Pez}_{\mathrm{L}}\right)$ en la capa superior
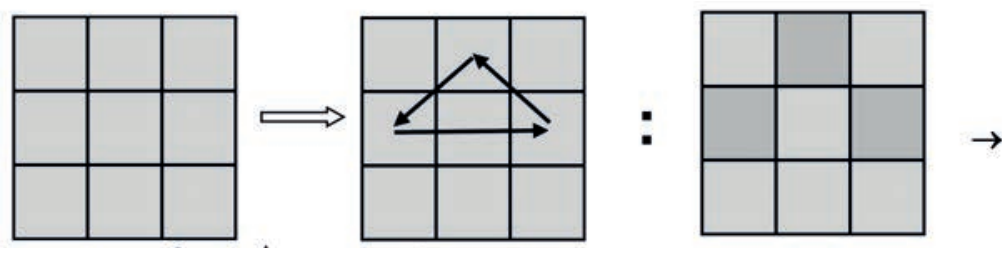

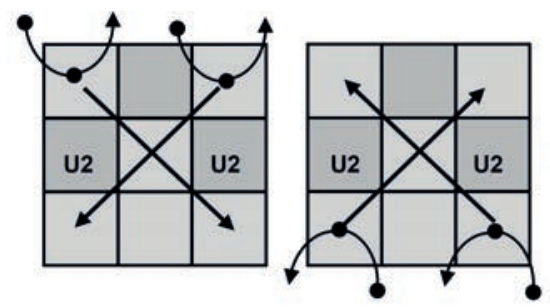

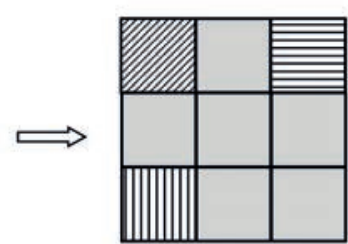

PezL

\subsubsection{Acción de $\hat{\mathbf{c}}\left(\mathrm{Pez}_{\mathrm{L}}\right)$ sobre sus aristas iniciales}

$\hat{\mathbf{c}}\left(\mathbf{P e z}_{\mathrm{L}}\right)$ realiza una permutación en sentido antihorario de tres de las aristas distintas de la inferior (permanece invariante).

\subsubsection{Acción de $\hat{\mathbf{c}}\left(\mathrm{Pez}_{\mathrm{L}}\right)$ sobre sus vértices iniciales}

Todos los vértices rotan $180^{\circ}$ en la capa superior (U2) pero los distintos al superior derecho reciben torsiones horarias de tres giros (1.6.3.3).

\subsection{Construir/deconstruir las "Cruces ampliadas" a partir de} los Peces básicos

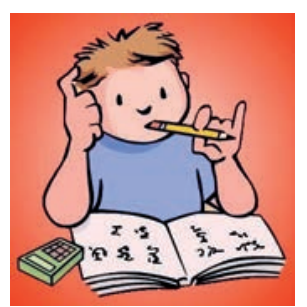

Esta sección se da para que el lector practique y observe la relación entre los dos peces básicos y los restantes 5 OLL del grupo de "las 7 cruces ampliadas.". En el desarrollo de esta actividad se deben utilizar los constructores/ deconstructores "canónicos" de los OLL ya expuestos; y observaremos que todos se pueden deconstruir empezando ese proceso con "el deconstructor canónico de $\mathrm{Pez}_{\mathrm{R} \text { ". }}$ La tarea del lector es comprobar usando el cubo de Rubik que los algoritmos dados luego de cada número es un constructor de la configuración allí mencionada; y además verificar que los diagramas ubicados más abajo también son correctos. 

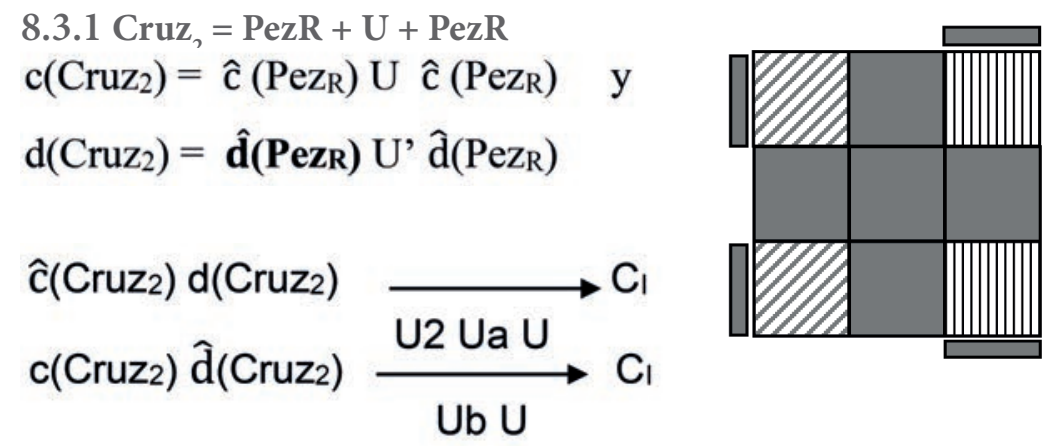

donde:

$$
\begin{aligned}
& \hat{\mathrm{c}}\left(\mathrm{Cruz}_{2}\right)=\mathrm{R} \text { ' U2 R2 U R2 U R2 U2 R', 7.4.1 } \\
& \text { d̂ }\left(\mathrm{Cruz}_{2}\right)=\text { R U2 R'2 U' R2 U' R2 U2 R, 7.4.1 } \\
& \mathrm{Ua}=\mathrm{M} 2 \mathrm{U}^{\prime} \mathrm{M} \quad \mathrm{U} 2 \quad \text { M' U' }^{\prime} \text { 2, 2.5.1.2. } \text { y } \\
& \mathrm{Ub}=\mathrm{M} 2 \mathrm{U} \text { M U2 M' U M2, 2.5.2.2. }
\end{aligned}
$$

8.3.2 Camaleón $=\mathrm{Pez}_{\mathrm{L}}+\mathrm{Pez}_{\mathrm{R}}$

$\mathrm{c}($ Camaleón $)=\hat{\mathrm{c}}(\mathrm{Pez}) \hat{\mathrm{c}}(\mathrm{Pez}) \quad \mathrm{y}$

$\mathrm{d}($ Camaleón $)=\hat{\mathbf{d}}($ PezR $) \widehat{d}\left(\operatorname{Pez}_{\mathrm{L}}\right)$

ĉ(Camaleón) d(Camaleón)

c(Camaleón) $\widehat{d}$ (Camaleón)

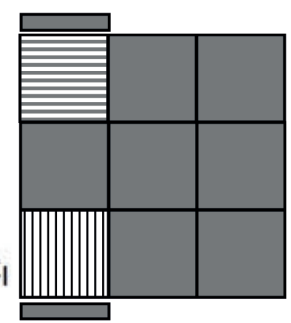

donde:

$$
\begin{aligned}
& \widehat{\mathrm{c}}(\text { Camaleón })=\left(\mathrm{F}^{\prime}\right. \text { ' F' r) (U R U’ r'), 7.6.1 } \\
& \widehat{\mathrm{d}} \text { (Camaleón) }=\text { (r U R' U') (r' F R F'), 7.6.1 } \\
& \mathrm{Ab}=\mathrm{R} 2 \mathrm{~B} 2 \mathrm{R} 2 \quad \mathrm{R}^{\prime} \mathrm{F} \mathrm{R}^{\prime} \quad \mathrm{B} 2 \quad \mathrm{R} \mathrm{F}^{\prime} \mathrm{R}, 2.6 .2 .2 \text {. y } \\
& \mathrm{Aa}=\mathrm{R}^{\prime} \mathrm{F} \text { R' } \quad \text { B2 } \quad \text { R F' R } \quad \text { R2 B2 R2, 2.6.1.2 }
\end{aligned}
$$




$$
\begin{aligned}
& \text { 8.3.3 Pajarita }=\mathrm{Pez}_{\mathrm{r}}+\mathrm{U}+\mathrm{Pez}_{\mathrm{D}} \\
& \mathrm{c}(\text { Pajarita })=\hat{\mathrm{c}}\left(\mathrm{Pez}_{\mathrm{L}}\right) \mathrm{U} \hat{\mathrm{c}}\left(\mathrm{Pez}_{\mathrm{R}}\right) \quad \mathrm{y} \\
& \mathrm{d}(\text { Pajarita })=\hat{\mathbf{d}}\left(\text { PezR }_{\mathbf{R}} \mathrm{U}^{\prime}, \hat{\mathrm{d}}\left(\mathrm{Pez}_{\mathrm{L}}\right)\right. \\
& \hat{c} \text { (Pajarita) d(Pajarita) } \\
& \longrightarrow \mathrm{U}^{\prime} \mathrm{J}_{\mathrm{I}} \mathrm{C}^{\prime} \\
& c(\text { Pajarita) } \hat{\mathrm{d}} \text { (Pajarita) }
\end{aligned}
$$

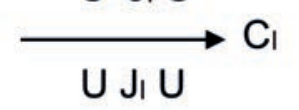

donde

$\widehat{c}($ Pajarita $)=$ r U R' U' r' (F R F'), 7.9.1

$\widehat{\mathrm{d}}$ (Pajarita) $=($ F R' F') r U R U' r', 7.9.1, y

$\mathrm{J}_{\mathrm{I}}=\mathrm{B}^{\prime}$ U B' R2 F D' F D $\quad$ F2 R2 B2, 4.4.2.

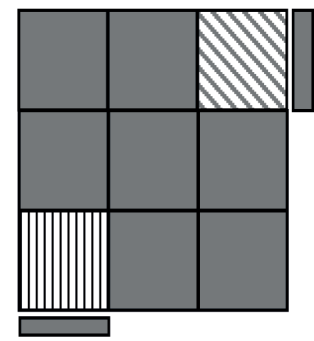

$$
\begin{aligned}
& \text { 8.3.4 Botella }=\text { Pez }_{\mathrm{r}}+\mathrm{U} 2+\mathrm{Pez}_{\mathrm{p}} \\
& \mathrm{c}(\text { Botella })=\hat{\mathrm{c}}\left(\mathrm{Pez}_{\mathrm{L}}\right) \mathrm{U} 2 \hat{\mathrm{c}}\left(\mathrm{Pez}_{\mathrm{R}}\right) \quad \mathrm{y} \\
& \mathrm{d}(\text { Botella })=\hat{\mathbf{d}}(\text { PezR }) \mathrm{U} 2 \hat{\mathrm{d}}\left(\mathrm{Pez}_{\mathrm{L}}\right)
\end{aligned}
$$$$
\text { c (Botella) d(Botella) }
$$$$
c \text { (Botella) } \hat{\mathrm{d}} \text { (Botella) }
$$

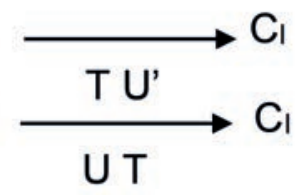

donde

$\hat{\mathrm{c}}($ Botella) $=\mathrm{R}$ U2 R $\quad$ D R' U2 R D' R2, 7.7.2 d̂ (Botella) = R2 DR' U2 RD' R' U2 R', 7.7.2, y T=RU R' U' R' FR2 U'R' U'RU R'F', 6.4.2

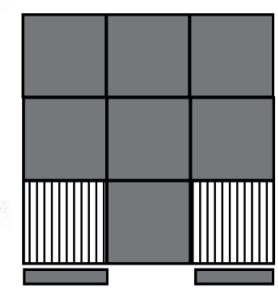

$$
\begin{aligned}
& \text { 8.3.5 } \text { Cruz }_{1}=\text { Pajarita }+\mathrm{U} 2+\mathrm{Pez}_{\mathrm{p}} \\
& \mathrm{c}\left(\mathrm{Cruz}_{1}\right)=\hat{\mathrm{c}}(\text { Pajarita }) \mathrm{U} 2 \hat{\mathrm{c}}\left(\text { Pez }_{\mathrm{R}}\right) \quad \mathrm{y} \\
& \mathrm{d}\left(\mathrm{Cruz}_{1}\right)=\hat{\mathrm{d}}\left(\text { Pez }_{\mathrm{R}}\right) \mathrm{U} 2 \hat{\mathrm{d}} \text { (Pajarita) }
\end{aligned}
$$




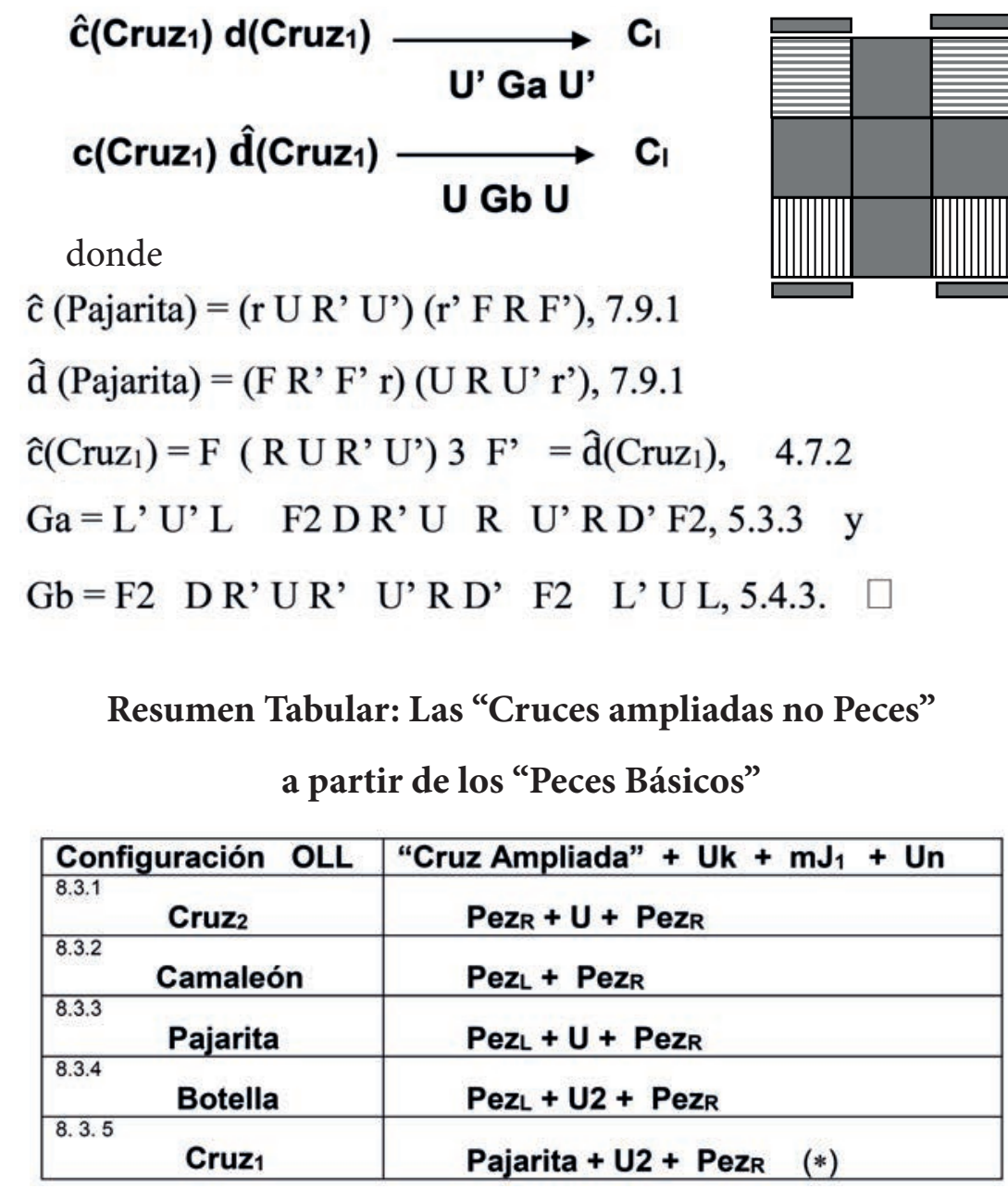

(*): Nótese que la Pajarita se construye a partir de peces básicos 


\section{CAPítulo \\ NUEVE}




\section{Doce Mini J ampliadas Optimizando Tiempo en La Resolución del Cubo de Rubik: Parte $1^{\text {a }}$}

Hasta el momento, sehan tratado en estelibrolas configuraciones OLL en formas de $\mathrm{T}\left(\mathrm{T}_{1}\right.$ y $\left.\mathrm{T}_{2}\right)$, la primera barra horizontal $\left(\mathrm{I}_{1}\right)$, la $1^{\text {a }}$ mini J (Mini $\left.\mathrm{J}_{1}\right)$, la $1^{\text {a }}$ mini $\mathrm{L}\left(\right.$ Mini $\left.\mathrm{L}_{1}\right)$ y las Siete "Cruces Ampliadas". Las restantes configuraciones OLL serán tratadas en este y en los tres capítulos siguientes.

\subsection{Clasificación de las restantes configuraciones OLL}

Las configuraciones OLL restantes, las que centrarán nuestra atención desde ahora, se pueden clasificar en mini J ampliadas, mini L ampliadas ${ }^{28}$, Barras Horizontales ampliadas y las Puntuales ampliadas.

\subsubsection{Las Mini J ampliadas:}

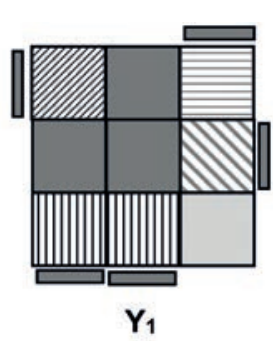
son aquellas donde se observa una mini J normal ó rotada con algunos (3) de sus stickers bien orientados de la capa superior. "Tienen dos y solo dos aristas bien orientadas y tales aristas son consecutivas". Se pueden deconstruir ${ }^{29}$ iniciando ese proceso con el deconstructor canónico de Mini $\mathbf{J}_{1}$ (o uno congruente) y usando además el deconstructor canónico de una "Cruz Ampliada" a precisar en su momento.

Comentario 1. Las Mini $\mathrm{J}$ ampliadas de más fácil construcción se diseñan iniciando ese proceso con el constructor de una "Cruz Ampliada" y operándolo luego con el constructor de mini $\mathrm{J}_{1}$ :

"Cruz Ampliada" + Mini $\mathrm{J}_{1}$

Mini J ampliada

28 Estas mini L ampliadas pueden ser también tratadas como mini L ampliadas al ejecutar sobre esas mini 1 ampliadas una rotación o movimiento U'.

29 Esta afirmación y las que más abajo planteamos en el mismo sentido son en realidad el objetivo central de este y del próximo capítulo. 


\subsubsection{Las Mini L ampliadas:}

son aquellas donde se observa una mini L

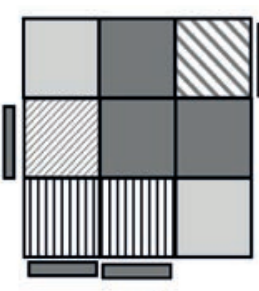
normal ó rotada con algunos (3) de sus stickers bien orientados de la capa superior. "Tienen dos y solo dos aristas bien orientadas y tales aristas son consecutivas". Se pueden deconstruir iniciando ese proceso con el deconstructor canónico de Mini $\mathrm{L}_{1}$ (o uno congruente) y usando además el $\mathbf{W}_{1} \mathbf{s}$ deconstructor canónico de una "Cruz Ampliada" a precisar en su momento.

Comentario 2. Las Mini L ampliadas de más fácil construcción se diseñan iniciando ese proceso con el constructor de una "Cruz Ampliada" y operándolo luego con el constructor de mini $L_{1}$

"Cruz Ampliada" + Mini $L_{1} \longrightarrow \quad$ Mini L ampliada

\subsubsection{Las Barras Centrales Ampliadas:}

son aquellas que No son Mini J ni Mini L

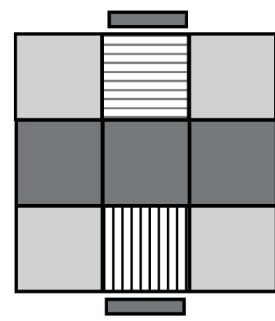
ampliadas, pero con algunos (3) de sus stickers bien orientados se forma una barra central (horizontal ó vertical). "Tienen dos y solo dos aristas bien orientadas y tales aristas son opuestas". Se pueden deconstruir iniciando ese proceso con el deconstructor canónico de $\mathrm{T}_{1}$ (o uno congruente) y usando además el H deconstructor canónico de una "Cruz Ampliada" a precisar en su momento

Comentario 3. Las Barras Centrales ampliadas de más fácil construcción se diseñan iniciando ese proceso con el constructor de una "Cruz Ampliada" y operándolo luego con el constructor de $\mathrm{T}_{1}$ :

"Cruz Ampliada" + $\mathrm{T}_{1} \longrightarrow$ Barra Central Ampliada 


\subsubsection{Las configuraciones Puntuales:}

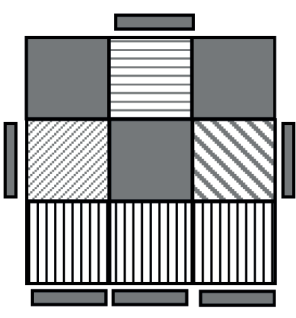

son las que no exhiben mini $\mathrm{J}$ o mini $\mathrm{L}$ alguna y tampoco son Barras Horizontales. "Ninguna de sus aristas está bien orientada".

Se pueden deconstruir iniciando ese proceso con el deconstructor $\mathbf{V}_{1}$ canónico de la "X" (o uno congruente), configuración que trataremos en el último capítulo, y usando además el deconstructor canónico de una "Cruz Ampliada" a

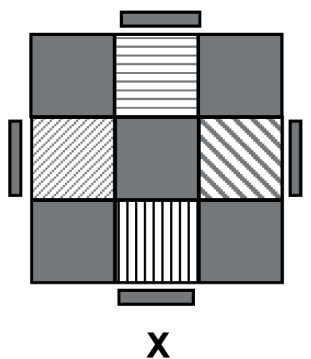
precisar en su momento.

Estos elementos teóricos y los estudiados en los capítulos anteriores constituyen:

\section{El "Método reducido de Fridrich" para la solución de la Última capa del cubo de Rubik}

Las configuraciones $T$, Mini $\mathrm{J}_{1}$, Mini $\mathrm{L}_{1}$ y Las cruces ampliadas $\mathrm{OLL}$, junto con los $21 \mathrm{PLL}$ ya conocidos y las rápidas deconstrucciones arriba mencionadas constituyen en el libro el "Método reducido de Fridrich" para la solución de la última capa del cubo de Rubik.

Para trabajar estas configuraciones primero se construyen con su constructor canónico y luego se deconstruyen iniciando el proceso con uno de los deconstructores antes indicados de acuerdo a la clase (Mini J, Mini L, Barra Horizontal o Puntual) a la cual pertenezca

30 El constructor canónico de " $\mathrm{X}$ " es su deconstructor canónico y está dado como: $\hat{c}(X)=$ (MU MU MU MU) (MU M'U M'U M'U) $=$ (MU) 4 (MU') $4=\hat{d}(X)$ 
9.2 Cinco primeras "Mini J ampliadas"

9.2.1 PezAL ${ }_{\mathrm{I}}=\mathrm{Cruz}_{2}+\mathrm{U} 2+\mathrm{mJ}{ }_{1}+\mathrm{U} 2$ :

El pez de aletas largas con cola a la izquierda ${ }^{31}$

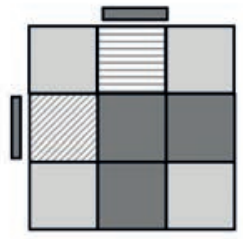

$$
\begin{aligned}
& \text { c }(\text { PezALI) }=\text { M U' M' U2 M U' M' } \\
& d(\text { PezALI })=U 2 \hat{d}\left(m^{\prime}\right) \text { U2 } \hat{d}\left(\text { Cruz }_{2}\right)
\end{aligned}
$$

PezAL

$$
\begin{aligned}
\text { donde } \hat{\mathrm{d}}\left(\mathrm{mJ}_{1}\right) & =\mathrm{F}(\mathrm{R} \text { U R' U') } 2 \text { F', 5.1.1; y } \\
\hat{\mathrm{d}}\left(\mathrm{Cruz}_{2}\right) & =\mathrm{R} \text { U2 R'2 } \quad \text { U' R2 U' R2 U2 R, 7.4.1. }
\end{aligned}
$$

9.2.2 $\square_{\mathrm{I}}=$ Botella $+\mathrm{U} 2+\mathrm{mJ}_{1}$ :

El "Cuadrado a Izquierda"

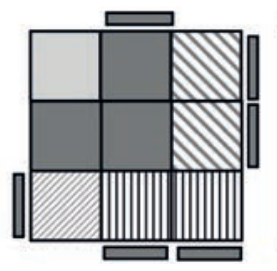

$$
\hat{c}\left(\square_{I}\right)=\mathbf{M} \hat{\mathbf{d}}\left(\text { PezL) } \mathbf{M}^{\prime}=I^{\prime} \mathbf{U}^{\prime} \mathbf{L}^{\prime} \mathbf{U}^{\prime} \quad \mathbf{L}^{\prime} \mathbf{U} \mathbf{2} \mathbf{I}\right.
$$

$\mathbf{d}\left(\square_{1}\right)=\hat{d}\left(m^{\prime} J_{1}\right) \mathbf{U} \hat{d}($ Botella)

l' = ML': movimiento parecido al movimiento L' solo que con I' se rotan simultáneamente las capas L' y su intermedia contigua $\mathrm{M}$ (sentido antihorario $90^{\circ}$ )

$\square$ I

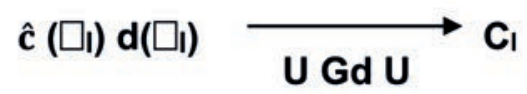

donde $\hat{\mathrm{d}}\left(\mathrm{mJ}_{1}\right)=\mathrm{F}$ ( R U R' U') 2 F', 5.1.1;

d (Botella)=R2 DR' U2 RD' R' U2 R', 7.7.2; y

Gd = RUR' F2 D' L U' L' U L'D F2, 5.5.3.

31 A la configuración hay que aplicarle una rotación de $180^{\circ}$ (ó U2) para obtener la Mini J (eso, de paso, simplifica la memorización de su constructor canónico). 
9.2.3 $\mathrm{mZ}_{1}=\operatorname{Mini} Z(2,2,1,0)=\mathrm{Pez}_{\mathrm{L}}+\mathrm{mJ}_{1}$ :

La Primera Mini $\mathrm{Z}^{32}$

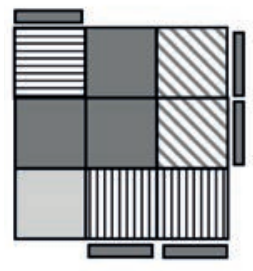

$\hat{c}\left(m Z_{1}\right)=M$ c (PezR) M' = r U2 R' U'R U' r'

$d\left(m Z_{1}\right)=\hat{d}\left(m^{\prime} J_{1}\right) \hat{d}($ PezL $)$

$\mathbf{r}=\mathbf{R M}$ : movimiento parecido al movimiento $\mathrm{R}$ solo que con " $r$ " se rotan simultáneamente las capas $R$ y su intermedia contigua $\mathrm{M}$ (sentido horario $90^{\circ}$ )

$m Z_{1}$

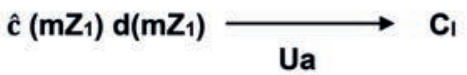

donde $\mathrm{d}\left(\mathrm{mJ}_{1}\right)=\mathrm{F}$ ( R U R' U') 2 F', 5.1.1;

$\widehat{\mathrm{d}}\left(\right.$ PezL $\left._{\mathrm{L}}\right)=$ L' U' L U' L' U2 L, 8.2.2; y

$\mathrm{Ua}=\mathrm{M} 2 \mathrm{U}^{\prime} \mathrm{M} \quad \mathrm{U} 2 \quad \mathrm{M}^{\prime} \mathrm{U}^{\prime} \mathrm{M} 2,2.5 .1 .2$.

9.2.4 $\mathrm{P}_{2}=\mathrm{P}(2,1,1,0)=$ Camaleón $+\mathrm{mJ}_{1}$ :

La Segunda $P$

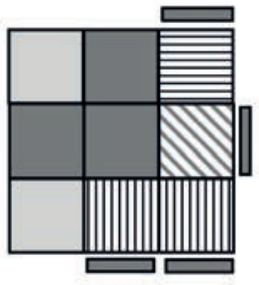

$\mathbf{P}_{2}$
$\hat{c}\left(P_{2}\right)=L$ d̂ $\left(T_{1}{ }^{S}\right)$ U' L' = L (F' L' U' L U F) U' L' $d\left(P_{2}\right)=\hat{d}\left(\mathbf{m J}_{1}\right) \hat{d}$ (Camaleón)

donde $\hat{\mathrm{d}}\left(\mathrm{mJ}_{1}\right)=\mathrm{F}$ ( R U R' U') 2 F', 5.1.1;

d̂ (Camaleón) = (r U R' U') (r' F R F'), 7.6.1; y

$\mathrm{N}_{\mathrm{I}}=\left(L^{\prime} \mathrm{U}^{\prime} \mathrm{R}^{\prime} \quad \mathrm{U} 2\right.$ L U'R) 2 U, 4.5.2.

32 Se le da este nombre a la configuración pues cuando se rota la capa superior $90^{\circ}$ sentido horario se observa una $\mathrm{Z}$ en la parte superior de dicha capa, pero esta $\mathrm{Z}$ es pequeña (existe también una $\mathrm{Z}$ grande) 
9.2.5 $\mathrm{CY}_{1}=$ Cuasi $_{1}(0,2,1,1)=\mathrm{Cruz}_{1}+\mathrm{U}+\mathrm{mJ}_{1}+\mathrm{U}^{\prime}$ :

\section{La $1^{\text {a }}$ Cuasi $Y^{33}$}

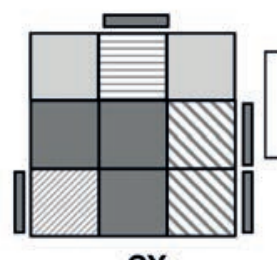

$$
\hat{c}\left(C Y_{1}\right)=M^{\prime} \hat{c}\left(T_{2}\right) U^{\prime} M=M^{\prime}\left(F R^{\prime} F^{\prime} \quad R U R U^{\prime} R^{\prime} U^{\prime}\right) M
$$
$\mathbf{d}\left(\mathrm{CY}_{1}\right)=\mathbf{U} \hat{\mathrm{d}}\left(\mathbf{m J ^ { \prime }}\right) \mathbf{U}^{\prime} \hat{\mathrm{d}}\left(\mathrm{Cruz}_{1}\right)$

$\mathrm{CY}_{1}$

$$
\hat{c}\left(C_{1}\right) d\left(C Y_{1}\right) \underset{U^{\prime} F U^{\prime}}{\longrightarrow} C_{1}
$$

donde $\mathrm{d}\left(\mathrm{mJ}_{1}\right)=\mathrm{F}$ ( R U R' U') 2 F', 5.1.1;

$\hat{\mathrm{d}}\left(\mathrm{Cruz}_{1}\right)=\mathrm{F}(\mathrm{R}$ U R' U')3 F', 4.7.2; y

$F=\left(R^{\prime} U R U^{\prime}\right)$ R2 (F'U'FU RFR'F') R2 U', 6.3.2

Síntesis tabular OLL “Canónica” (9.2.1 $\rightarrow$ 9.2.5)

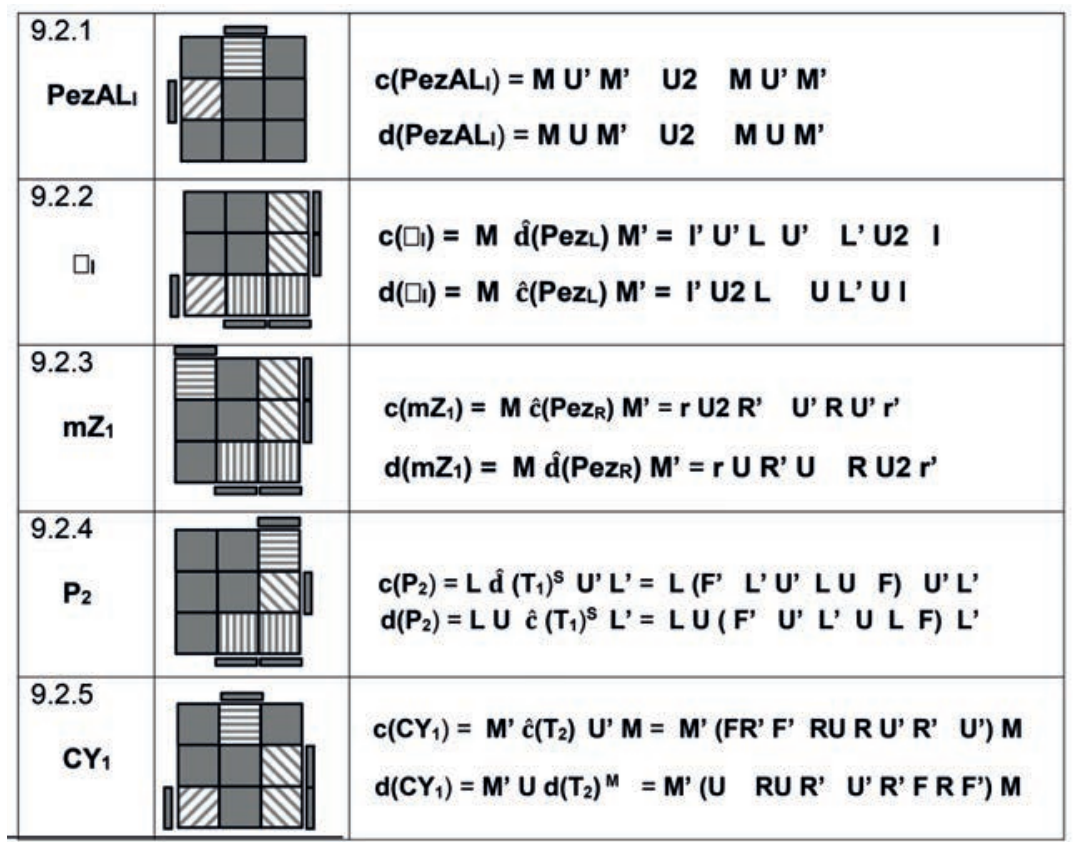

33 La configuración es una Y excepto el sticker de la arista derecha, la cual no está bien orientada. De ahí su nombre. Nótese que su deconstructor no comienza en $\mathrm{d}_{\left(\mathrm{mJ}_{1}\right)}$ ) sino con un movimiento U. 
9.2.6 Las inversas de esas cinco configuraciones

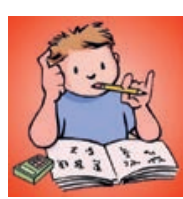

Todas estas inversas son configuraciones que se van a tratar en los próximos dos capítulos, además, el lector puede anticipar conocimiento a partir de su propia experiencia.

\begin{tabular}{|c|c|c|}
\hline & Inversa & constructor/deconstructor de la Inversa \\
\hline PezALI & PezALd & 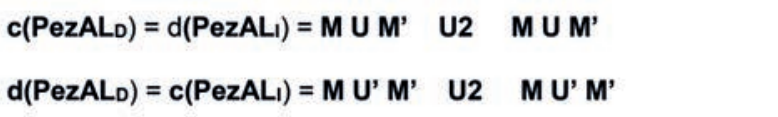 \\
\hline 미 & $\mathrm{mS}_{1}$ & 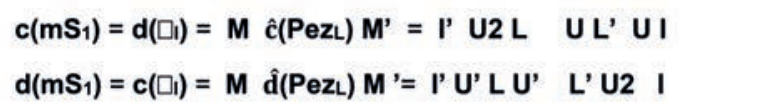 \\
\hline $\mathbf{m} \mathbf{Z}_{1}$ & पo & $\begin{array}{l}c(\square v)=d\left(m Z_{1}\right)=M d\left(P e z_{R}\right) M^{\prime}=r \text { U R' U R U2 r' } \\
d(\square v)=c\left(m Z_{1}\right)=M \hat{c}\left(P e z_{R}\right) M^{\prime}=r \text { U2 R' } U^{\prime} R U^{\prime} r^{\prime}\end{array}$ \\
\hline $\mathbf{P}_{\mathbf{2}}$ & Z & $\begin{array}{l}c(Z)=d\left(P_{2}\right)=L U \hat{c}\left(T_{1}\right)^{s} L^{\prime}=L U\left(F^{\prime} U^{\prime} L^{\prime} U L F\right) L^{\prime} \\
d(Z)=c\left(P_{2}\right)=L \hat{d}\left(T_{1}\right)^{s} U^{\prime} L^{\prime}=L\left(F^{\prime} L^{\prime} U^{\prime} L U F\right) U^{\prime} L^{\prime}\end{array}$ \\
\hline $\mathrm{CY}_{1}$ & $\begin{array}{l}\mathrm{CY}_{1}-1 \\
(\sim \mathrm{Z})\end{array}$ & $\begin{array}{l}c\left(C Y_{1}-1\right)=d\left(C Y_{1}\right)=M^{\prime} U d\left(T_{2}\right) M=M^{\prime}\left(U R U R^{\prime} U^{\prime} R^{\prime} F R F^{\prime}\right) M \\
d\left(C Y_{1}-1\right)=c\left(C Y_{1}\right)=M^{\prime} \hat{c}\left(T_{2}\right) U^{\prime} M=M^{\prime}\left(F R^{\prime} F^{\prime} \text { RU R U' R' U') } M\right.\end{array}$ \\
\hline
\end{tabular}

La semejanza $(\sim)$ acá es con una congruente a la configuración allí nominada

\subsection{Otras Cuatro "Mini J Ampliadas"}

$$
\text { 9.3.1 } \mathrm{mS}_{2}=\text { Mini } S(1,2,1,1)=\text { Camaleón }+U+\mathrm{mJ}_{1}+\mathrm{U}^{\prime} \text { : }
$$

La $2^{\text {a }}$ Mini $S^{34}$

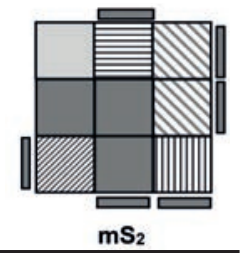

$\hat{c}\left(m S_{2}\right)=\hat{c}\left(T_{1}{ }^{s}\right) U \hat{c}\left(T_{1}{ }^{s}\right)=\left(F^{\prime} U^{\prime} L^{\prime} U L F\right) U$ (F' U'L'UL $\left.F\right)$ $d\left(\mathrm{mS}_{2}\right)=\mathbf{U} \hat{\mathrm{d}}\left(\mathrm{mJ}_{1}\right) \mathbf{U}$ ' $\mathbf{d}$ (Camaleón)

$$
\hat{c}\left(m S_{2}\right) d\left(m S_{2}\right) \underset{U G b}{\longrightarrow} C_{1}
$$

34 La primera Mini S es la simétrica de la primera Mini Z. Se le da el nombre de Mini S pues al rotarse $90^{\circ}$ sentido horario o antihorario tal configuración (movimientos U ó U') se obtiene una $S$ pequeña (existe también $S$ grande a estudiar en el capítulo 11). 
donde $\hat{\mathrm{d}}\left(\mathrm{mJ}_{1}\right)=\mathrm{F}\left(\mathrm{R} U \mathrm{R}^{\prime} \mathrm{U}^{\prime}\right) 2$ F', 5.1.1;

d̂ (Camaleón) = (r U R' U') (r' F R F'), 7.6.1; y

$\mathrm{Gb}=\mathrm{F} 2$ D R'U R' U'R D' F2 L'U L, 5.4.3.

9.3.2 $\mathrm{mJ}_{2}=\operatorname{Mini} \mathrm{J}(3,1,2,0)=$ Botella $+\mathrm{U}+\mathrm{mJ}_{1}$ :

La Segunda Mini J

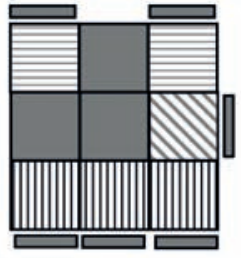

$\mathbf{m} \mathbf{J}_{2}$ $\hat{\mathbf{c}}\left(\mathbf{m J}_{\mathbf{2}}\right)=\mathrm{M} \mathrm{c}_{1}\left(\mathrm{Cruz}_{1}\right) \mathrm{M}^{\prime}=\mathbf{r} \mathbf{U}\left(\mathbf{R}^{\prime} \mathbf{U} \mathbf{U}^{\prime}\right) 2 \mathbf{U}^{\prime} \mathbf{r}^{\prime}$ $d\left(m J_{2}\right)=\hat{d}\left(m J_{1}\right) U ' \hat{d}$ (Botella)

$r=R M$ : movimiento parecido al movimiento $R$ solo que con " $r$ " se rotan simultáneamente las capas $R$ y su intermedia contigua $\mathrm{M}$ (sentido horario $90^{\circ}$ )

$$
\hat{c}\left(m J_{2}\right) d\left(m J_{2}\right)
$$

$\mathbf{C}_{1}$

Ro

donde $\mathbf{c}_{1}\left(\mathbf{C r u z}_{1}\right)=\mathrm{RU}$ (R' U R U')2 U' R', 4.8.1;

d̂ $\left(\mathrm{mJ}_{1}\right)=\mathrm{F}(\mathrm{R}$ U R' U' $) 2$ F', 5.1.1;

d (Botella)= R2 DR' U2 RD' R' U2 R', 7.7.2; y

$\mathrm{R}_{\mathrm{D}}=\mathrm{R}^{\prime}$ U2 R U2 R' FRU R' U'R' F' R2 U', 5.7.1

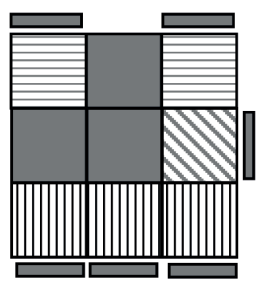

9.3.3 $\mathrm{Y}_{\mathrm{I}}=$ Camaleón $+\mathrm{U}^{\prime}+\mathrm{mJ}_{1}$ :

"Y inclinada a la Izquierda"

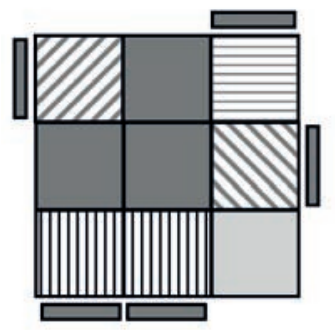

$$
\begin{aligned}
& \widehat{c}\left(Y_{1}\right)=\left(F R^{\prime} F^{\prime}\right) U^{\prime} \text { (FR F' R') U R } \\
& d\left(Y_{1}\right)=\hat{d}\left(m^{\prime} J_{1}\right) U \hat{d}(\text { Camaleón) }
\end{aligned}
$$

$\hat{\mathbf{c}}\left(\mathbf{Y}_{\mathbf{I}}\right) \mathbf{d}\left(\mathbf{Y}_{\mathrm{I}}\right)$

$\mathbf{Y}_{1}$

donde $\widehat{\mathrm{d}}\left(\mathrm{mJ}_{1}\right)=\mathrm{F}\left(\mathrm{R} U \mathrm{R}^{\prime} \mathrm{U}^{\prime}\right) 2$ F', 5.1.1;

d (Camaleón) = (r U R' U') (r' F R F'), 7.6.1; y

$\mathrm{Z}=(\mathrm{M} 2 \mathrm{U}$ M2 U) (MU2 M2 U2 MU2), 2.8.2. 


$$
\begin{aligned}
& \text { 9.3.4 W }=\mathrm{Cruz}_{2}+\mathrm{U}^{\prime}+\mathrm{mJ}_{1} \text { : } \\
& \text { "La W" }
\end{aligned}
$$

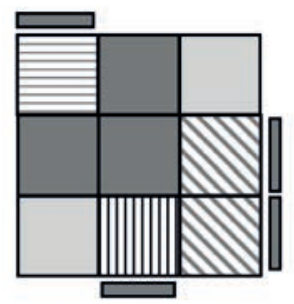

$$
\begin{aligned}
\hat{c}(W) & =\left(F R^{\prime} F^{\prime}\right)(R U R U)\left(R^{\prime} U^{\prime} R U^{\prime} R^{\prime}\right) \\
d(W) & =\hat{d}\left(m J_{1}\right) U \hat{d}\left(C r u z_{2}\right)
\end{aligned}
$$

W

\begin{tabular}{|c|c|}
\hline $\begin{array}{r}9.3 .1 \\
\mathrm{mS}_{2}\end{array}$ & $\begin{array}{l}c\left(m S_{2}\right)=\hat{c}\left(T_{1} s\right) U \hat{c}\left(T_{1} s\right)=\left(F^{\prime} U^{\prime} L^{\prime} U L L\right) U\left(F^{\prime} U^{\prime} L^{\prime} U L F\right) \\
d\left(m S_{2}\right)=\hat{d}\left(T_{1} s\right) U^{\prime} \hat{d}\left(T_{1} s\right)=\left(F^{\prime} L^{\prime} U^{\prime} L U F\right) U^{\prime}\left(F^{\prime} L^{\prime} U^{\prime} L U F\right)\end{array}$ \\
\hline $\begin{array}{r}9.3 .2 \\
\mathbf{m J}_{2}\end{array}$ & $\begin{array}{l}c\left(m J_{2}\right)=r U\left(R^{\prime} U R U^{\prime}\right) 2 U^{\prime} r^{\prime} \\
d\left(m J_{2}\right)=r U\left(U R^{\prime} U^{\prime} R\right) 2 U^{\prime} r^{\prime}\end{array}$ \\
\hline $\begin{array}{r}9.3 .3 \\
Y_{1}\end{array}$ & $\begin{array}{l}c\left(Y_{1}\right)=\left(F R^{\prime} F^{\prime}\right) U^{\prime}\left(F R F^{\prime} R^{\prime}\right) \text { U R } \\
d\left(Y_{1}\right)=R^{\prime} U^{\prime}\left(R R^{\prime} F^{\prime}\right) \text { U (FR F') }\end{array}$ \\
\hline $\begin{array}{r}9.3 .4 \\
\mathrm{~W}\end{array}$ & $\begin{array}{l}c(W)=\left(F R^{\prime} F^{\prime}\right)(R U R U)\left(R^{\prime} U^{\prime} R U^{\prime} R^{\prime}\right) \\
d(W)=\left(R U R^{\prime} \quad U R\right)\left(U^{\prime} R^{\prime} U^{\prime} R^{\prime}\right)\left(F R F^{\prime}\right)\end{array}$ \\
\hline
\end{tabular}

donde $\hat{\mathrm{d}}\left(\mathrm{Cruz}_{2}\right)=\mathrm{R}$ U2 R'2 U' R2 U' R2 U2 R, 7.4.1; y

$$
\text { Gd=RUR' F2 D' L U' L' U L' D F2, 5.5.3. }
$$

Síntesis tabular OLL “Canónica” (9.3.1 $\rightarrow$ 9.3.4)

\subsubsection{Las inversas de esas cuatro configuraciones}

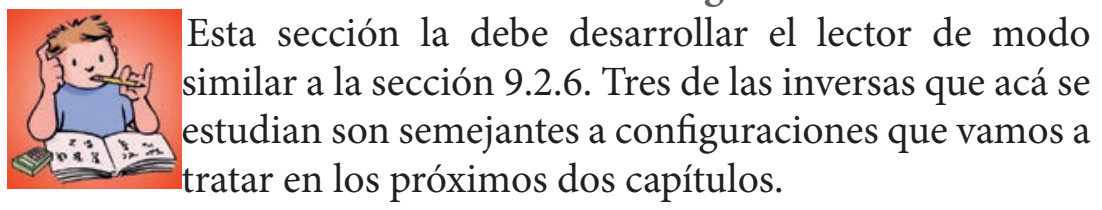

\begin{tabular}{|c|c|c|}
\hline & Inversa & constructor/deconstructor de la Inversa \\
\hline $\mathrm{mS}_{2}$ & $\mathbf{m S _ { 2 } - 1}$ & $\mathbf{c}\left(\mathrm{mS}_{2}{ }^{-1}\right)=\mathrm{d}\left(\mathrm{mS}_{2}\right)=\left(F^{\prime} L^{\prime} U^{\prime}\right.$ LUF) U' (F' L'U' LUF) \\
& $\left(\sim \mathrm{mS}_{1}\right)$ & $\mathbf{d}\left(\mathrm{mS}_{2}{ }^{-1}\right)=\mathbf{c}\left(\mathrm{mS}_{2}\right)=\left(F^{\prime} U^{\prime} L^{\prime}\right.$ UL F) U (F' U'L' \\
\hline
\end{tabular}




\begin{tabular}{|c|c|c|}
\hline $\mathrm{mJ}_{2}$ & $\begin{array}{l}\mathrm{mJ}_{2}-1 \\
\left(\sim \mathrm{mJ}_{2}\right)\end{array}$ & $\begin{array}{l}c\left(m J_{2}{ }^{-1}\right)=d\left(m J_{2}\right)=r U\left(U R^{\prime} U^{\prime} R\right) 2 \quad U^{\prime} r^{\prime} \\
d\left(m J_{2}{ }^{-1}\right)=c\left(m J_{2}\right)=r U\left(R^{\prime} U R U^{\prime}\right) 2 \quad U^{\prime} r^{\prime}\end{array}$ \\
\hline $\mathbf{Y}_{1}$ & $\mathrm{~L}_{1}$ & $\begin{array}{l}c\left(L_{1}\right)=d\left(Y_{1}\right)=R^{\prime} U^{\prime}\left(R R^{\prime} F^{\prime}\right) U\left(F F^{\prime}\right) \\
d\left(L_{1}\right)=c\left(Y_{1}\right)=\left(F R^{\prime} F^{\prime}\right) U^{\prime}\left(F R F^{\prime} R^{\prime}\right) \cup R\end{array}$ \\
\hline w & $\begin{array}{l}W^{-1} \\
\left(\sim q_{2}\right)\end{array}$ & $\begin{array}{l}c\left(W^{-1}\right)=d(W)=\left(R U R^{\prime} \quad U R\right)\left(U^{\prime} R^{\prime} U^{\prime} R^{\prime}\right)\left(F R F^{\prime}\right) \\
d\left(W^{-1}\right)=c(W)=\left(F R^{\prime} F^{\prime}\right) \quad(R U R U)\left(R^{\prime} U^{\prime} R U^{\prime} R^{\prime}\right)\end{array}$ \\
\hline
\end{tabular}

La semejanza $(\sim)$ acá es con una congruente a la configuración allí nominada

\subsection{Las restantes Tres "Mini J Ampliadas"}

Esta sección la debe desarrollar el lector como una buena práctica. En concreto, damos los constructores canónicos de configuraciones de esta clase, su tarea consiste en construirlas usando tales constructores (a partir del cubo en su estado original) y deconstruirlas como en los casos ya ilustrados, es decir, partiendo del deconstructor de Mini $J_{1}$ y luego retornar el cubo a su estado original.

9.4.1 Cometa $_{2}=$ Cometa $(1,1,1,1)$ :

\section{La Segunda Cometa}

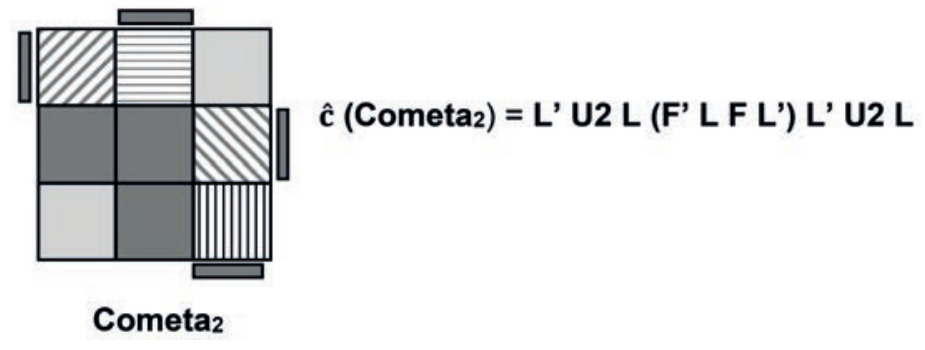

9.4.2 $\mathrm{CY}_{2}=$ Cuasi $\mathrm{Y}_{1}(1,1,0,2)$ :

\section{La Segunda Cuasi Y}

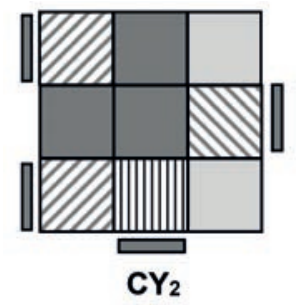

$\hat{c}\left(C Y_{2}\right)=\left(R U^{\prime} R^{\prime}\right) \cup\left(R U^{\prime} R^{\prime}\right)\left(F R^{\prime} F^{\prime} R\right) 2$ 
9.4.3 $\mathrm{mJ}_{3}=\operatorname{Mini} \mathrm{J}(2,3,1,0)$ :

La tercera Mini J.

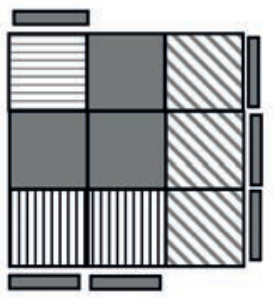

$$
\hat{c}\left(m J_{3}\right)=F\left(R \quad R^{\prime} U^{\prime} R\right) F^{\prime}\left(r \quad U R^{\prime} U^{\prime} r^{\prime}\right)
$$

$\mathbf{m J}_{3}$

Síntesis tabular OLL “Canónica” (9.4.1 $\rightarrow$ 9.4.3)

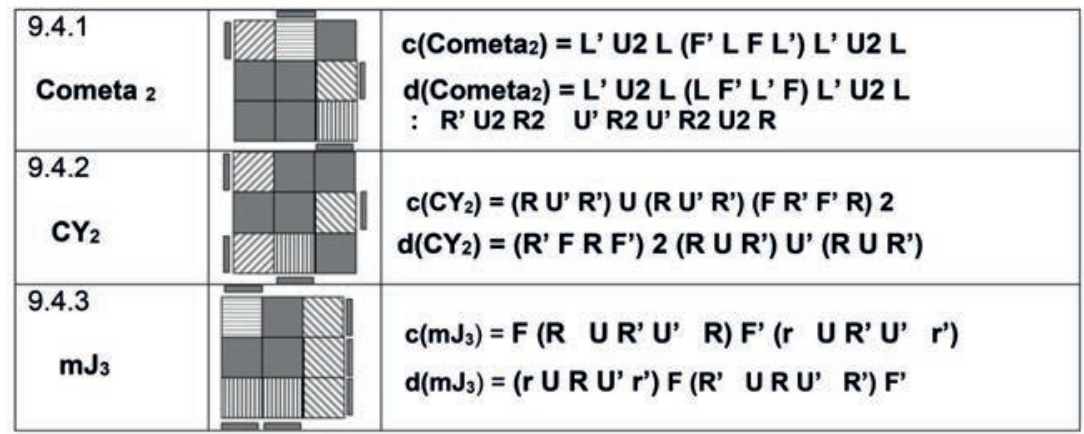

\subsubsection{Las inversas de esas tres configuraciones}

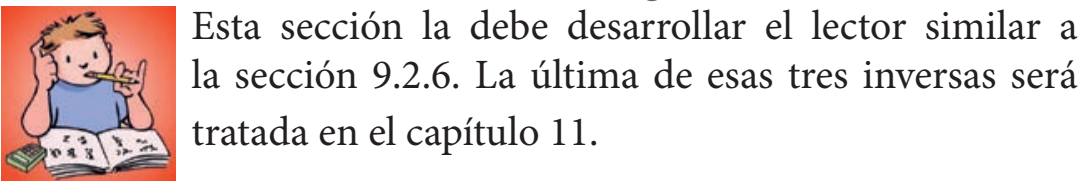

\begin{tabular}{|c|c|c|}
\hline & Inversa & constructor/deconstructor de la Inversa \\
\hline Cometa2 & $\begin{array}{l}\text { Cometa2 }^{-1} \\
\text { ( Cometa1, } \\
\text { 1.9.1) }\end{array}$ & $\begin{array}{l}c\left(\text { Cometa }^{-1}\right)=d\left(\text { Cometa }_{2}\right)=L^{\prime} \text { U2 L } \quad \text { (L F' L' F) L' } \\
d\left(\text { Cometa }^{-1}\right)=c\left(\text { Cometa }_{2}\right)=L^{\prime} \text { U2 L } \quad\left(F^{\prime} L^{\prime} L^{\prime}\right) \quad L^{\prime}\end{array}$ \\
\hline $\mathrm{CY}_{2}$ & $\begin{array}{l}\mathrm{CY}_{2}{ }^{-1} \\
\left(\sim \mathrm{T}_{2}, 2.1 .1\right)\end{array}$ & $\begin{array}{l}c\left(C Y_{2}-^{-1}\right)=d\left(C Y_{2}\right)=\left(R^{\prime} F R F^{\prime}\right) 2 \quad\left(R U R^{\prime}\right) U^{\prime}(R U \\
d\left(C Y_{2}{ }^{-1}\right)=c\left(C Y_{2}\right)=\left(R U^{\prime} R^{\prime}\right) U\left(R U^{\prime} R^{\prime}\right) \quad\left(F R^{\prime} F^{\prime} R\right)\end{array}$ \\
\hline $\mathrm{mJ}_{3}$ & $I_{3}$ & $\begin{array}{l}c\left(I_{3}\right)=d\left(m J_{3}\right)=\left(r U R U^{\prime} r^{\prime}\right) F\left(R^{\prime} \quad U R U^{\prime} R^{\prime}\right) F^{\prime} \\
d\left(I_{3}\right)=c\left(m J_{3}\right)=F\left(R \quad U R^{\prime} U^{\prime} R\right) F^{\prime}\left(r \quad U R^{\prime} U^{\prime} r^{\prime}\right)\end{array}$ \\
\hline
\end{tabular}

La semejanza $(\sim)$ acá es con una congruente a la configuración allí nominada 
9.5 Práctica: Las Mini J "Ampliadas" a partir de las "Cruces ampliadas"

\begin{tabular}{|c|c|}
\hline Configuración OLL & "Cruz Ampliada" + Uk + $\mathrm{mJ}_{1}+\mathrm{Un}$ \\
\hline $\mathbf{m Z _ { 1 }}$ & $P_{e z L}+m_{J}$ \\
\hline 9.2 .5 & $\operatorname{Cruz}_{1}+U+m J_{1}+U^{\prime}$ \\
\hline $\mathrm{mJ}_{3}$ & $\mathrm{Cruz}_{2}+\mathrm{mJ}_{1}$ \\
\hline 9.3 .4 & $\operatorname{Cruz}_{2}+U^{\prime}+m_{1}$ \\
\hline PezALl & $\mathrm{Cruz}_{2}+\mathrm{U}_{2}+\mathrm{mJ}_{1}+\mathrm{U}_{2}$ \\
\hline 9.2 .4 & Camaleón + $\mathrm{mJ}_{1}$ \\
\hline 9.3 .3 & Camaleón + U' + mJ1 \\
\hline $\mathbf{m S}_{2}$ & Camaleón + U+ $m J_{1}+U^{\prime}$ \\
\hline $\mathbf{m J}_{\mathbf{2}}$ & Botella + U + mJ 1 \\
\hline $\mathrm{CY}_{2}$ & Botella + U' + $\mathrm{mJ}_{1}$ \\
\hline 9.2 .2 & Botella + U2 + mJ \\
\hline $\begin{array}{l}9.4 .1 \quad \text { Cometa2 } \\
\end{array}$ & Pajarita + U' + $\mathrm{mJ}_{1}+\mathrm{U}^{\prime}$ \\
\hline
\end{tabular}




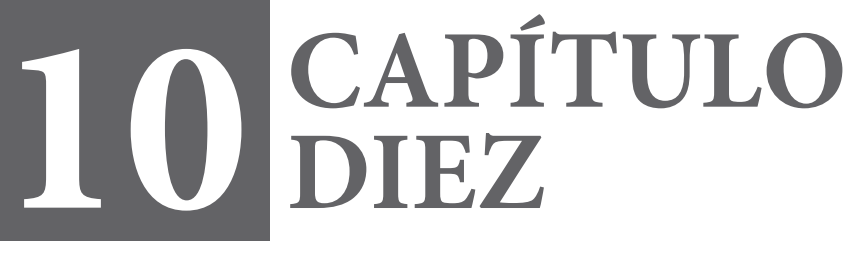




\section{Doce Mini L ampliadas o simétricas de las Mini J ampliadas Optimizando Tiempo en La Resolución del Cubo de Rubik: Parte 2a}

Del capítulo anterior sabemos que estas configuraciones son aquellas donde se observa una mini L normal o rotada diseñada con tres de sus stickers bien orientados de la capa superior. "Tienen dos y solo dos aristas bien orientadas y tales aristas son consecutivas". Acá comprobaremos que estas se pueden deconstruir iniciando ese proceso con "el deconstructor canónico de Mini $\mathrm{L}_{1 \text { " }} \mathrm{y}$ usando además un OLL congruente al "deconstructor canónico de una "Cruz Ampliada"' a precisar en su momento.

Las Mini L ampliadas de más fácil construcción se diseñan iniciando ese proceso con el constructor de una "Cruz Ampliada" y operándolo luego con el constructor de mini $\mathrm{L}_{1}$ :

\section{"Cruz Ampliada" + Mini $L_{1} \longrightarrow$ Mini L ampliada}

\subsection{Cinco primeras "Mini L ampliadas"}

10.1.1 $\operatorname{PezAL}_{\mathrm{D}}=\mathrm{Cruz}_{2}+\mathrm{mL}_{1}+\mathrm{U} 2$ :

El pez de aletas largas con cola a la derecha

(Simétrico del "pez de aletas largas con cola a la izquierda" PezALI, 9.2.1).

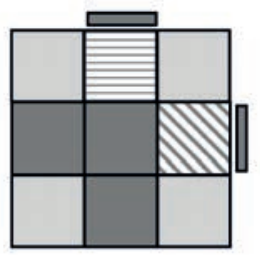

PezALo

$$
\begin{aligned}
& \hat{c}(\text { PezALD })=M \text { U M' U2 M U M' }=\hat{d}(\text { PezAL } 1) \\
& \text { d(PezALD })=U 2 \hat{d}\left(m L_{1}\right) \hat{d}\left(C_{r u z}\right)
\end{aligned}
$$

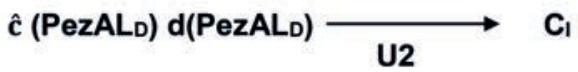


donde $\hat{\mathrm{d}}\left(\mathrm{mL}_{1}\right)=\mathrm{F}^{\prime}$ (L' U' L U ) 2 F, 7.1.2; y

$\widehat{\mathrm{d}}\left(\mathrm{Cruz}_{2}\right)=\mathrm{R}$ U2 R'2 U' R2 U' R2 U2 R, 7.4.2.

10.1.2 $\square_{\mathrm{D}}=$ Botella $+\mathrm{U} 2+\mathrm{mL}_{1}$ :

El "Cuadrado a derecha" 35

(Simétrico del "Cuadrado a Izquierda" $\square_{\mathrm{I}}$, 9.2.2).

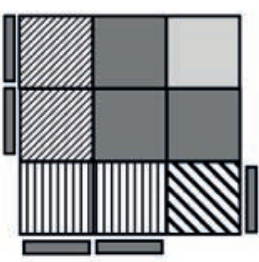

口v

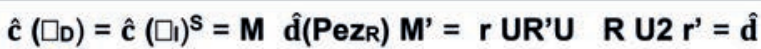
$\left(m Z_{1}\right)$

$\mathbf{d}(\square \mathrm{D})=\hat{\mathbf{d}}\left(\mathrm{mL}_{1}\right) \mathbf{U} \mathbf{2} \hat{\mathbf{d}}($ Botella)

$\mathbf{r}=\mathbf{R M}$ : movimiento parecido al movimiento $\mathrm{R}$ solo que con " $r$ " se rotan simultáneamente las capas $R$ y su intermedia contigua $\mathrm{M}$ (sentido horario $90^{\circ}$ )

donde $\hat{\mathrm{d}}\left(\mathrm{mL}_{1}\right)=\mathrm{F}^{\prime}\left(\mathrm{L}^{\prime} \mathrm{U}^{\prime} \mathrm{L} \mathrm{U}\right) 2 \mathrm{~F}, 7.1 .2$;

d̂ (Botella)=R2 DR' U2 RD' R' U2 R', 7.7.2; y

$\mathrm{J}_{\mathrm{I}}=\mathrm{B}^{\prime}$ U B' R2 F D' F D F2 R2 B2, 4.4.2.

10.1.3 $\mathrm{mS}_{1}=\operatorname{Mini} \mathrm{S}(2,0,1,2)=\mathrm{Pez}_{\mathrm{R}}+\mathrm{mL}_{1}$ :

La Primera Mini $S^{36}$

( Simétrica de la Primera Mini Z, 9.2.3).

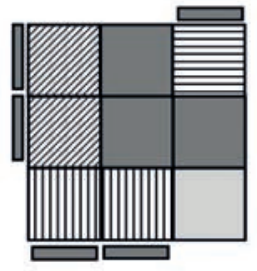

$\mathrm{mS}_{1}$

$\hat{c}\left(m S_{1}\right)=\hat{c}\left(m Z_{1}{ }^{s}\right)=\hat{d}\left(\square_{I}\right)=M \hat{c}\left(P e Z_{L}\right) M^{\prime}=l^{\prime} U 2 L \quad U$ L'U I

$\mathbf{d}\left(\mathbf{m S}_{1}\right)=\hat{\mathbf{d}}\left(\mathbf{m L}_{1}\right) \hat{\mathbf{d}}($ PezR $)$

l' = ML': movimiento parecido al movimiento L' solo que con I' se rotan simultáneamente las capas L' y su intermedia contigua $\mathrm{M}\left(\right.$ sentido antihorario $90^{\circ}$ )

$$
\hat{c}\left(m S_{1}\right) d\left(m S_{1}\right) \longrightarrow C_{1}
$$

35 Este cuadrado resulta ser el inverso de la Primera Mini Z (9.2.3).

36 Se le da este nombre a la configuración pues cuando se rota la capa superior $90^{\circ}$ sentido horario o antihorario se observa una $\mathrm{S}$ en dicha capa, pero esta es pequeña (existe también una $\mathrm{S}$ grande a tratar en el próximo capítulo). Esta Mini S resulta ser la inversa del "Cuadrado a Izquierda" (9.2.2). 
donde $\hat{\mathrm{d}}\left(\mathrm{mL}_{1}\right)=\mathrm{F}^{\prime}$ ( L' U' L U) $2 \mathrm{~F}, \mathrm{~g}$ 7.1.2;

d $($ PezR $)=$ R U R' U R U2 R', 8.1.2; y

$\mathrm{Ub}=\mathrm{M} 2 \mathrm{U}$ M U2 M' U M2, 2.5.2.2

10.1.4 $\mathrm{q}_{2}=\mathrm{q}(2,0,1,1)=$ Camaleón $+\mathrm{U} 2+\mathrm{mL}_{1}$ :

La Segunda q (Simétrica de $\mathrm{P}_{2}, 9.2 .4$ )

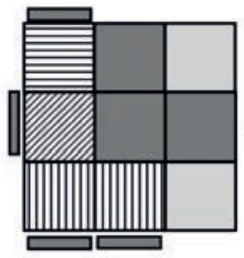

$\hat{c}\left(q_{2}\right)=\hat{c}\left(P_{2}\right)^{s}=R^{\prime} \hat{d}\left(T_{1}\right) U R=R^{\prime}\left(F R U R^{\prime} U^{\prime} F^{\prime}\right) U R$ $\mathbf{d}\left(q_{2}\right)=\hat{d}\left(m L_{1}\right) \mathbf{U} 2 \hat{d}($ Camaleón)

$\mathbf{q}_{2}$

donde $\widehat{\mathrm{d}}\left(\mathrm{mL}_{1}\right)=\mathrm{F}^{\prime}\left(\mathrm{L}^{\prime} \mathrm{U}^{\prime} \mathrm{L} \mathrm{U}\right) 2 \mathrm{~F}, 7.1 .2$;

d (Camaleón) = (r U R' U') (r' F R F'), 7.6.2; y

$\mathrm{Z}=(\mathrm{M} 2 \mathrm{U}$ M2 $\mathrm{U})(\mathrm{MU} 2 \mathrm{M} 2 \mathrm{U} 2 \mathrm{MU} 2)$, 2.8.2.

10.1.5 $\mathrm{CY}_{3}=$ Cuasi $_{3}(0,1,1,2)=\mathrm{Cruz}_{1}+\mathrm{U}+\mathrm{mL}_{1}+\mathrm{U}$ :

La $3^{\text {a }}$ Cuasi Y $^{37}$ (Simétrica de la Primera Cuasi Y, 9.2.5).

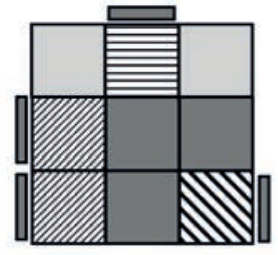

$$
\begin{aligned}
\hat{c}\left(C Y_{3}\right) & =\hat{c}\left(C Y_{1}\right)^{S}=M^{\prime} \hat{c}\left(T_{2}\right)^{s} U M \\
& =M^{\prime}\left(F F^{\prime} L^{\prime} L^{\prime} U^{\prime} L^{\prime} U L\right. \\
d\left(C Y_{3}\right) & =U^{\prime} \hat{d}\left(m L_{1}\right) U^{\prime} \hat{d}\left(C r u z_{1}\right)
\end{aligned}
$$

$\mathrm{CY}_{3}$

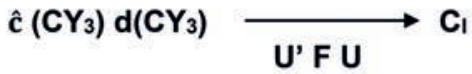

donde $\hat{\mathrm{d}}\left(\mathrm{mL}_{1}\right)=\mathrm{F}^{\prime}\left(\mathrm{L}^{\prime} \mathrm{U}^{\prime} \mathrm{L} \mathrm{U}\right) 2 \mathrm{~F}, 7.1 .2$;

$\widehat{\mathrm{d}}\left(\mathrm{Cruz}_{1}\right)=\mathrm{F}(\mathrm{R}$ U R' U')3 F', 4.7.2; y

$F=\left(R^{\prime}\right.$ U R U') R2 (F'U'FU RFR'F') R2 U', 6.3.2.

37 La configuración es una Y excepto el sticker de la arista izquierda, la cual no está bien orientada.

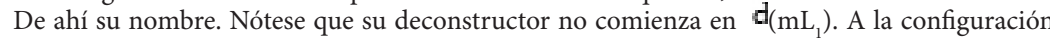
hay que aplicarle un movimiento U' para obtener la Mini L (eso, de paso, simplifica la memorización de su constructor canónico). 
Síntesis tabular OLL “Canónica” $(9.2 .1 \rightarrow 9.2 .5)^{38}$

\begin{tabular}{|c|c|}
\hline $\begin{array}{l}10.1 .1 \\
\text { PezALo }\end{array}$ & $\begin{array}{l}c(\text { PezALD })=\hat{d}(\text { PezALL })=M M^{\prime} \quad \text { U2 } M U^{\prime} M^{\prime} \\
d(\text { PezALD })=\hat{c}(\text { PezALI })=M U^{\prime} M^{\prime} \text { U2 } M U^{\prime} M^{\prime}\end{array}$ \\
\hline $\begin{array}{r}10.1 .2 \\
\square \text { D }\end{array}$ & 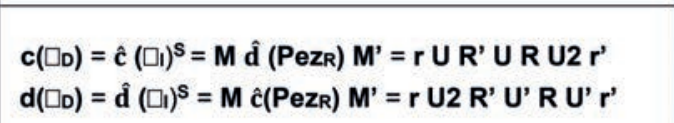 \\
\hline $\begin{array}{r}10.1 .3 \\
\mathrm{mS}_{1}\end{array}$ & $\begin{array}{l}c\left(m S_{1}\right)=\hat{d}\left(\square_{1}\right)=M \hat{c}\left(P e z_{L}\right) M^{\prime}=I^{\prime} U_{2} L \quad U L^{\prime} U^{\prime} \\
d\left(m S_{1}\right)=\hat{c}\left(\square_{I}\right)=M \hat{d}\left(P e z_{L}\right) M^{\prime}=I^{\prime} U^{\prime} L U^{\prime} L^{\prime} U_{2} I\end{array}$ \\
\hline $\begin{array}{c}10.1 .4 \\
\mathbf{q}_{2}\end{array}$ & $\begin{array}{l}c\left(q_{2}\right)=\hat{c}\left(P_{2}\right)^{s}=R^{\prime} \hat{d}\left(T_{1}\right) U R=R^{\prime}\left(F R U R^{\prime} U^{\prime} F^{\prime}\right) U R \\
d\left(q_{2}\right)=\hat{d}\left(P_{2}{ }^{s}\right)=R^{\prime} U^{\prime} \hat{c}\left(T_{1}\right) R=R^{\prime} U^{\prime}\left(F \cup R U^{\prime} R^{\prime} F^{\prime}\right) R\end{array}$ \\
\hline $\begin{array}{r}10.1 .5 \\
\mathrm{CY}_{3}\end{array}$ & $\begin{array}{l}c\left(C Y_{3}\right)=\hat{c}\left(Y_{1}\right)^{s}=M^{\prime} \hat{c}\left(T_{2}\right)^{s} U M=M^{\prime}\left(F^{\prime} L F \quad L^{\prime} U^{\prime} L^{\prime} U L \text { U) } M\right. \\
d\left(C Y_{3}\right)=\hat{d}\left(Y_{1}\right)^{s}=M^{\prime} U^{\prime} \hat{d}\left(T_{2}\right)^{s} M=M^{\prime}\left(U^{\prime} L^{\prime} U^{\prime} L U L \text { F'L'F) } M\right.\end{array}$ \\
\hline
\end{tabular}

10.1.6 Las inversas de esas cinco configuraciones

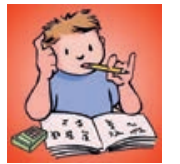

Esta sección la debe desarrollar el lector similar a la sección 9.2.6. Una de estas inversas, la $\mathrm{S}$, la vamos a tratar mejor en el próximos capítulo.

\begin{tabular}{|c|c|c|c|c|c|}
\hline & Inversa & \multicolumn{4}{|c|}{ Constructor/deconstructor de la Inversa } \\
\hline PezALD & $\begin{array}{l}\text { PezALI } \\
(9.2 .1)\end{array}$ & 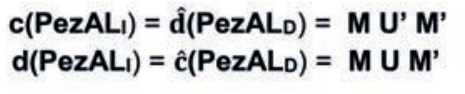 & $\begin{array}{l}\text { U2 } \\
\text { U2 }\end{array}$ & $\begin{array}{l}M U^{\prime} M^{\prime} \\
M \quad M^{\prime}\end{array}$ & \\
\hline पo & $\begin{array}{c}m \mathbf{Z}_{1} \\
(9.2 .3)\end{array}$ & $\begin{array}{l}c\left(m Z_{1}\right)=\hat{d}(\square v)=M \hat{c}\left(P e Z_{R}\right) M^{\prime}= \\
d\left(m Z_{1}\right)=\hat{c}(\square v)=\hat{c}\left(\square_{1}\right)^{s}=M \hat{d}(P e z\end{array}$ & U2 & $\begin{array}{l}U^{\prime} R U^{\prime} r \\
=\text { r U R' U }\end{array}$ & R U2 \\
\hline
\end{tabular}

\begin{tabular}{|c|c|c|}
\hline & Inversa & Constructor/deconstructor de la Inversa \\
\hline $\mathrm{mS}_{1}$ & $\begin{array}{c}\square 1 \\
(9.2 .2)\end{array}$ & 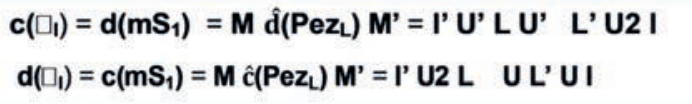 \\
\hline$q_{2}$ & $\mathbf{S}$ & 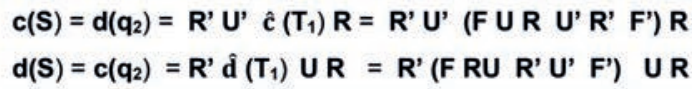 \\
\hline
\end{tabular}

38 Acá se usan todos los contructores y deconstructores de "La Síntesis tabular OLL "Canónica", Sección 9.2" y solamente se usan los de dicha tabla. En verdad esto facilita en demasía la memorización de los algoritmos OLL. 


\begin{tabular}{|c|c|c|}
\hline $\mathrm{CY}_{3}$ & $\begin{array}{l}\mathrm{CY}_{3}{ }^{-1} \\
(\sim \mathrm{S})\end{array}$ & $\begin{array}{l}c\left(C Y_{3}{ }^{-1}\right)=d\left(C Y_{3}\right)=M^{\prime} U^{\prime} \hat{d}\left(T_{2}\right)^{s} M=M^{\prime}\left(U^{\prime} L^{\prime} U^{\prime} L U L F^{\prime} L^{\prime} F\right) M \\
d\left(C Y_{3}{ }^{-1}\right)=c\left(C Y_{3}\right)=M^{\prime} \hat{c}\left(T_{2}\right)^{s} U M=M^{\prime}\left(F^{\prime} L F \text { L'U'L' } L^{\prime} \text { U) } M\right.\end{array}$ \\
\hline
\end{tabular}

La semejanza $(\sim)$ acá es con una congruente a la configuración allí nominada

\subsection{Otras cuatro "Mini L Ampliadas"}

10.2.1 $\mathrm{mZ}_{2}=$ Mini Z $(1,1,1,2)=$ Camaleón $+\mathrm{U}+\mathrm{mL}_{1}+\mathrm{U}$ :

\section{La $2^{\text {a }}$ Mini $Z^{39}$}

(Simétrica de la segunda Mini S, 9.3.1).

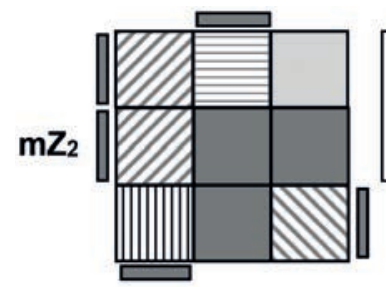

$$
\begin{aligned}
\hat{c}\left(m Z_{2}\right) & =\hat{c}\left(m S_{2}\right)^{s}=\hat{c}\left(T_{1}\right) U^{\prime} \hat{c}\left(T_{1}\right) \\
& =\left(F U R U^{\prime} R^{\prime} F^{\prime}\right) U^{\prime}\left(F U R U^{\prime} R^{\prime} F^{\prime}\right) \\
d\left(m Z_{2}\right) & =U^{\prime} d^{\prime}\left(m L_{1}\right) U^{\prime} \text { d̂ (Camaleón) }
\end{aligned}
$$

$\hat{\mathbf{c}}\left(m Z_{2}\right) d\left(m Z_{2}\right)$

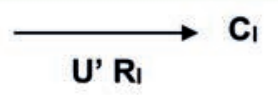

donde $\hat{\mathrm{d}}\left(\mathrm{mJ}_{1}\right)=\mathrm{F}$ (R U R' U')2 F', 5.1.1;

d (Camaleón) $=$ (r U R' U') (r' F R F'), 7.6.1; y

$$
\mathrm{R}_{\mathrm{I}}=\mathrm{L} \text { U2 L' U2 L F' L'U' L U L F L2 U, 6.1.2. }
$$

\subsection{2 $\mathrm{mL}_{2}=$ Mini L $(3,0,2,1)=$ Botella $+U^{\prime}+\mathrm{mL}_{1}$ :}

\section{La Segunda Mini L}

(Simétrica de la Segunda Mini J, 9.3.2)

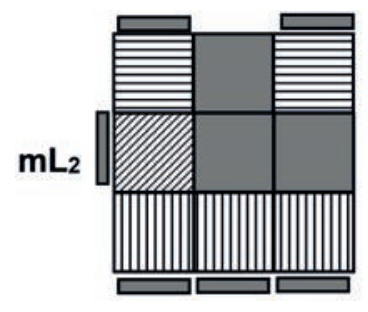

$$
\begin{aligned}
& \hat{c}\left(m L_{2}\right)=\hat{c}\left(m J_{2}\right)^{s}=I^{\prime} U^{\prime} \quad\left(L U^{\prime} L^{\prime} U\right) 2 \quad U ~ I \\
& d\left(m L_{2}\right)=\hat{d}\left(m L_{1}\right) U \text { d (Botella) }
\end{aligned}
$$

$$
\hat{\mathbf{c}}\left(\mathrm{mL}_{2}\right) \mathrm{d}\left(\mathrm{mL}_{2}\right)
$$

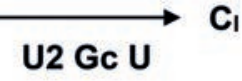

\footnotetext{
39 Se le da el nombre de Mini Z pues al rotarse $90^{\circ}$ sentido horario o antihorario tal configuración (movimientos $\mathrm{U}$ ó $\mathrm{U}^{\prime}$ ) se obtiene una $\mathrm{Z}$ pequeña (existe también $\mathrm{Z}$ grande a estudiar en el próximo capítulo).
} 
donde $\widehat{\mathrm{d}}\left(\mathrm{mL}_{1}\right)=\mathrm{F}^{\prime}$ (L' U' L U )2 F, 7.1.2;

d̂ (Botella) = R2 DR' U2 RD' R' U2 R', 7.7.2; y

$\mathrm{Gc}=\mathrm{F} 2 \quad \mathrm{D}^{\prime} \mathrm{L} \mathrm{U}^{\prime} \quad \mathrm{L} \quad \mathrm{U}^{\prime} \mathrm{L}^{\prime} \mathrm{D} \quad \mathrm{F} 2 \quad \mathrm{R} \mathrm{U}^{\prime} \mathrm{R}^{\prime}$, 5.6.3.

10.2.3 $\mathrm{Y}_{\mathrm{D}}=$ Camaleón $+\mathrm{U}^{\prime}+\mathrm{mL}_{1}$ :

"Y inclinada a la derecha"

(Simétrica de $\mathrm{Y}_{\mathrm{I}}$, 9.3.3)
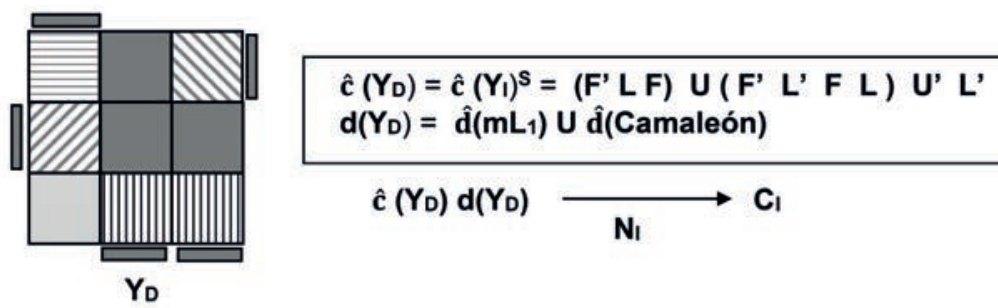

donde $\hat{\mathrm{d}}\left(\mathrm{mL}_{1}\right)=\mathrm{F}^{\prime}\left(\mathrm{L}^{\prime} \mathrm{U}^{\prime} \mathrm{L} \mathrm{U}\right) 2 \mathrm{~F}, 7.1 .2$;

d̂ (Camaleón) = (r U R' U') (r' F R F'), 7.6.1; y

$\mathrm{N}_{\mathrm{I}}=\left(L^{\prime} \mathrm{U}^{\prime}\right.$ ' U2 L U'R) $2 \mathrm{U}$, 4.5.2.

10.2.4 $\mathrm{W}^{\mathrm{S}}=\mathrm{Cruz}_{2}+\mathrm{U}^{\prime}+\mathrm{mL}_{1}$ :

“La Simétrica de W" (9.3.4)

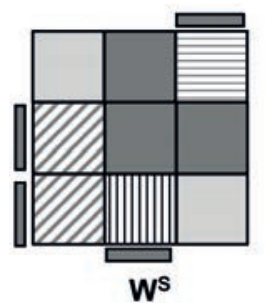

$\hat{c}\left(W^{s}\right)=\hat{c}(W)^{s}=\left(F^{\prime} L F\right) \quad\left(L^{\prime} U^{\prime} L^{\prime} U^{\prime}\right) \quad\left(L U L^{\prime} U L\right)$ $\mathbf{d}\left(W^{S}\right)=\hat{d}\left(m_{1}\right) \cup \hat{d}\left(C_{r u z}\right)$

donde $\hat{\mathrm{d}}\left(\mathrm{mL}_{\mathrm{l}}\right)=\mathrm{F}^{\prime}$ (L' U' L U) 2 F, 7.1.2;

d̂ (Cruz $)=$ R U2 R'2 U' R2 U' R2 U2 R, 7.4.2; y

$\mathrm{T}=\mathrm{RU} \mathrm{R}^{\prime} \mathrm{U}^{\prime} \mathrm{R}^{\prime} \quad$ FR2 U'R' U'RU R'F', 6.4.2 
Síntesis tabular OLL “Canónica” (10.2.1 $\rightarrow$ 10.2.4)

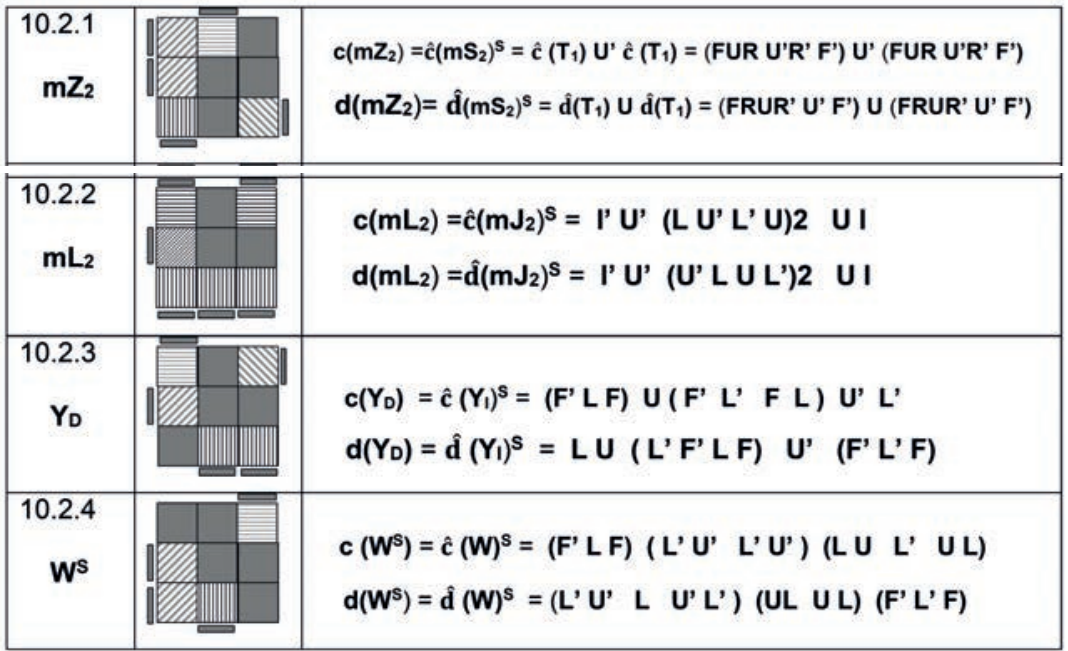

\subsection{5 as inversas de esas cuatro configuraciones}

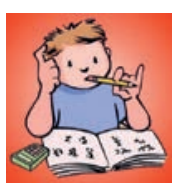
Esta sección la debe desarrollar el lector de modo similar a la sección 10.1.6. Una de estas inversas, la $\mathrm{J}_{1}$, se trata mejor en el próximo capítulo.

\begin{tabular}{|c|c|c|}
\hline & Inversa & Constructor/deconstructor de la Inversa \\
\hline $\mathbf{m Z}_{2}$ & 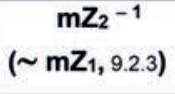 & $\begin{array}{l}c\left(m Z_{2}-1\right)=d\left(m Z_{2}\right)=\left(F R U R^{\prime} U^{\prime} F^{\prime}\right) U\left(F R \cup R^{\prime} U^{\prime} F^{\prime}\right) \\
d\left(m Z_{2}-1\right)=c\left(m Z_{2}\right)=\left(F \text { UR U'R' } F^{\prime}\right) U(F \text { U U U }\end{array}$ \\
\hline $\mathrm{mL}_{2}$ & $\begin{array}{l}\mathrm{mL}_{2}^{-1} \\
\left(\sim \mathrm{mL}_{2}\right)\end{array}$ & $\begin{array}{l}c\left(m L_{2}-1\right)=d\left(m L_{2}\right)=I^{\prime} U^{\prime}\left(U^{\prime} L L^{\prime}\right) 2 \quad U \mid \\
d\left(m L_{2}{ }^{-1}\right)=c\left(m L_{2}\right)=I^{\prime} U^{\prime}\left(L U^{\prime} L^{\prime} U\right) 2 \quad U \mid\end{array}$ \\
\hline$Y_{D}$ & $\mathbf{J}_{1}$ & $\begin{array}{l}c\left(J_{1}\right)=d\left(Y_{D}\right)=L U L^{\prime}\left(F^{\prime} L F \quad U^{\prime} F^{\prime} L^{\prime} F\right) \\
d\left(J_{1}\right)=c\left(Y_{D}\right)=\left(F^{\prime} L F \quad U F^{\prime} L^{\prime} F\right) L U^{\prime} L^{\prime}\end{array}$ \\
\hline $\mathbf{w}^{\mathbf{s}}$ & $\begin{array}{c}\text { W-S } \\
\left(\sim P_{2}, 9.2 .4\right)\end{array}$ & $\begin{array}{l}c\left(W^{-s}\right)=d\left(W^{s}\right)=\left(L^{\prime} U^{\prime} \quad L \quad U^{\prime} L^{\prime}\right)\left(U L \quad U L^{\prime}\right)\left(F^{\prime} L^{\prime} F\right) \\
d\left(W^{-s}\right)=c\left(W^{s}\right)=\left(F^{\prime} L F\right)\left(L^{\prime} U^{\prime} L^{\prime} U^{\prime}\right)\left(L U L^{\prime} U\right.\end{array}$ \\
\hline
\end{tabular}

La semejanza $(\sim)$ acá es con una congruente a la configuración allí nominada

\subsection{Las restantes Tres "Mini L Ampliadas".}

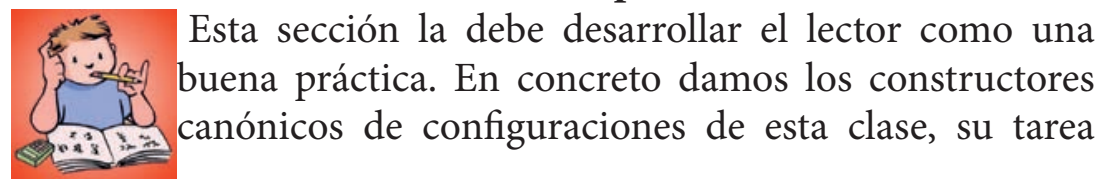


consiste en construirlas usando tales constructores (a partir del cubo en su estado original) y deconstruirlas como en los casos ya ilustrados, es decir, partiendo del deconstructor de Mini $\mathrm{L}_{1}$ y luego retornar el cubo a su estado original.

10.3.1 La Cometa ${ }_{2}{ }^{{ }}$" "Simétrica de la Cometa ${ }_{2}$ (9.4.1)

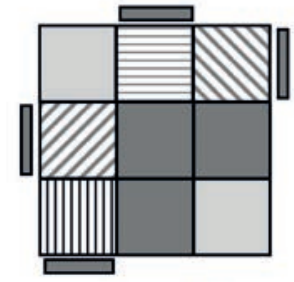

$$
\hat{c}\left(\text { Cometa }_{2}{ }^{\text {s }}\right)=\text { RU2 R' (F R' F' R) RU2 R' }
$$

Cometa $^{s}$

10.3.2 $\mathrm{CY}_{4}=$ Cuasi $_{3}(1,2,0,1)$ : "La Cuarta Cuasi Y" (Simétrica de la segunda Cuasi Y, 9.4.2)

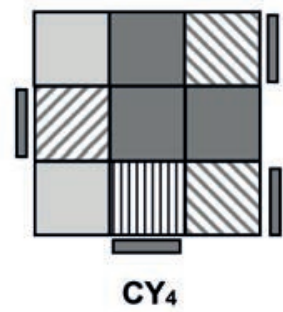

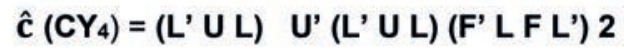

10.3.3 $\mathrm{mL}_{3}=$ Mini L $(2,0,1,3)$ : La tercera Mini L

(Simétrica de $3^{\text {a }}$ Mini J, 9.4.3)

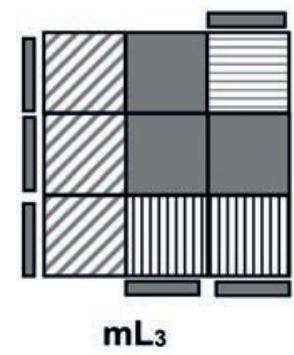

$$
\hat{c}\left(m_{3}\right)=F^{\prime}\left(L^{\prime} \quad U^{\prime} L U \quad L^{\prime}\right) \text { F (l' U' L U I) }
$$


Síntesis tabular OLL “Canónica” $(10.3 .1 \rightarrow 10.3 .3)$

\begin{tabular}{|c|c|}
\hline $\begin{array}{l}10.3 .1 \\
\text { Cometa }_{2} \mathrm{~s}\end{array}$ & $\begin{array}{l}c\left(\text { Cometa }_{2}{ }^{\mathrm{s}}\right)=\text { RU2 R' (F R' F' R) RU2 R' } \\
\mathrm{d}\left(\text { Cometa }_{2} \mathrm{~s}^{\mathrm{s}}\right)=\text { R U2 R' (R' F R F') R U2 R' }\end{array}$ \\
\hline $\begin{array}{c}10.3 .2 \\
\mathrm{CY}_{4}\end{array}$ & $\begin{array}{l}c\left(C Y_{4}\right)=\left(L^{\prime} U^{\prime}\right) U^{\prime}\left(L^{\prime} U L^{\prime}\right)\left(F^{\prime} L L^{\prime}\right) 2 \\
d\left(C Y_{4}\right)=\left(L F^{\prime} L^{\prime} F\right) 2\left(L^{\prime} U^{\prime} L\right) U\left(L^{\prime} U^{\prime} L\right)\end{array}$ \\
\hline $\begin{array}{r}10.3 .3 \\
\mathrm{~mL}_{3}\end{array}$ & 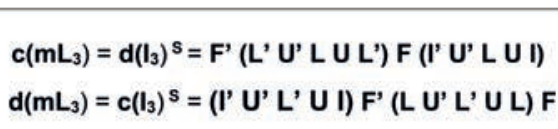 \\
\hline
\end{tabular}

\subsubsection{Las inversas de esas tres configuraciones}

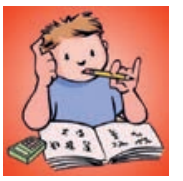

Esta sección la debe desarrollar el lector similar a la sección 10.1.6. La última de esas tres inversas, $\mathrm{I}_{3}$, será tratada en el próximo capítulo.

\begin{tabular}{|c|c|c|}
\hline & Inversa & Constructor/deconstructor de la Inversa \\
\hline $\mathrm{Cometa}_{2} \mathrm{~s}$ & $\begin{array}{l}\text { Cometa }_{2}-\mathrm{s} \\
\left(\sim \text { Cometa }_{1}{ }^{s}, 2.4 .1\right)\end{array}$ & 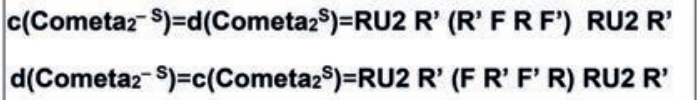 \\
\hline $\mathrm{CY}_{4}$ & $\begin{array}{c}\mathrm{CY}_{4}{ }^{-1} \\
\left(\sim \mathrm{T}_{2}, 2.1 .1\right)\end{array}$ & $\begin{array}{l}c\left(C Y_{4}{ }^{-1}\right)=d\left(C Y_{4}\right)=\left(L F^{\prime} L^{\prime} F\right) 2 \quad\left(L^{\prime} U^{\prime} L\right) U\left(L^{\prime} U^{\prime} L\right) \\
d\left(C Y_{4}{ }^{-1}\right)=c\left(C Y_{4}\right)=\left(L^{\prime} U L\right) \quad U^{\prime}\left(L^{\prime} U L\right) \quad\left(F^{\prime} L F L^{\prime}\right) 2\end{array}$ \\
\hline $\mathbf{m L}_{3}$ & $\mathrm{I}_{3} \mathrm{~S}$ & 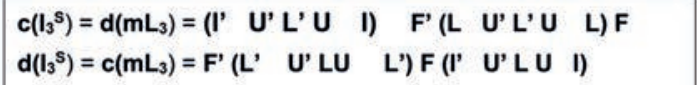 \\
\hline
\end{tabular}

La semejanza $(\sim)$ acá es con una congruente a la configuración allí nominada 
10.4 Práctica: Las Mini L "Ampliadas" a partir de las "Cruces ampliadas"

\begin{tabular}{|c|c|}
\hline Configuración OLL & "Cruz Ampliada" + U + mJ $J_{1}+U n$ \\
\hline $\mathrm{mS}_{1}$ & $P_{e z R}+m L_{1}$ \\
\hline 10.1 .5 & $\mathrm{Cruz}_{1}+U+\mathrm{mL}_{1}+U$ \\
\hline 10.3 .3 & $\mathrm{Cruz}_{2}+\mathrm{U} 2+\mathrm{mL}_{1}$ \\
\hline 10.2 .4 & $\mathrm{Cruz}_{2}+U^{\prime}+\mathrm{mL}_{1}$ \\
\hline PezALD & $\mathrm{Cruz}_{2}+\mathrm{mL}_{1}+\mathrm{U} 2$ \\
\hline 10.1 .4 & Camaleón $+\mathrm{U}_{2}+\mathrm{mL}_{1}$ \\
\hline 10.2 .3 & Camaleón + U' + mL1 \\
\hline$m Z_{2}$ & Camaleón $+U+m L_{1}+U$ \\
\hline $\mathrm{mL}_{2}$ & Botella + U' + $\mathrm{mL}_{1}$ \\
\hline $\mathrm{CY}_{4}$ & Botella $+\mathrm{U}+\mathrm{mL}_{1}$ \\
\hline 10.1 .2 & Botella $+\mathrm{U}_{2}+\mathrm{mL}_{1}$ \\
\hline 10.3.1 Cometa $2 \mathrm{~s}$ & Pajarita + U2 + $\mathrm{mL}_{1}+\mathrm{U}$ \\
\hline
\end{tabular}

10.5 Sección Complementaria:

La "X” y su simétrica a partir de los Peces de Aletas largas

$$
\begin{aligned}
& \mathbf{X}=\text { PezALd U2 PezALI } \\
& \mathbf{X}^{\mathbf{S}}=\text { PezAL, U2 PezALo } \sim \mathbf{X}
\end{aligned}
$$

Aprovechamos lo conocido hasta este momento sobre tales peces para generar la configuración "X", fundamental en la deconstrucción de las configuraciones puntuales (9.1.4.) a tratar en el capítulo 12. 


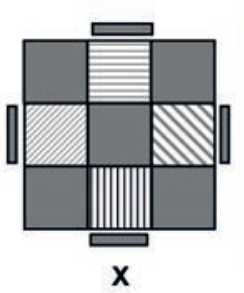

1" Forma ("Nueva") $c(X)=c($ PezALD) U2 $c($ PezALI) $d(X)=c(X)=c($ PezALD $)$ U2 c(PezALI)

$2^{\text {a }}$ Forma ("canónica"):

$\hat{c}(X)=($ MU MU MUMU) (M'U M'U M'U M'U) $=(M U) 4$ (M'U)4 $\hat{d}(X)=\hat{c}(X)=(M U) 4\left(M^{\prime}\right)^{4} 4$

(*) $\mathbf{c}($ PezALD $)=$ M U M' U2 M U M', 10.1.1.

c(PezAL 1$)=$ M U' M' U2 M U' M', 9.2.1.

Estas dos configuraciones no son congruentes pues mientras en la forma canónica todas sus aristas están bien posicionadas (mal orientadas), se observa que en la $1^{\text {a }}$ forma no sucede tal situación. No obstante, son configuraciones semejantes tal como lo puede comprobar el lector verificando la validez de estos dos diagramas:

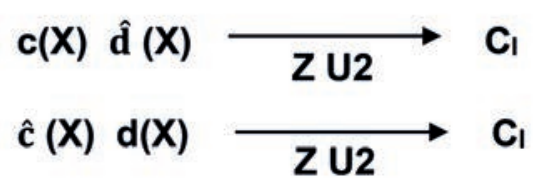

donde $\mathrm{Z}=(\mathrm{M} 2 \mathrm{U}$ M2 $\mathrm{U})$ (MU2 $\mathrm{M} 2 \mathrm{U} 2 \mathrm{MU}$ ), 2.8.2. 
11 CAPÍTULO 


\section{Doce Barras Centrales Ampliadas Optimizando Tiempo en La Resolución del Cubo de Rubik: Parte $3^{\mathrm{a}}$.}

Del capítulo 9 se sabe que estas configuraciones son aquellas que No son Mini J ni Mini L ampliadas, pero con algunos (3) de sus stickers bien orientados se forma una barra central (horizontal o vertical). "Tienen dos y solo dos aristas bien orientadas y tales aristas son opuestas".

De las doce barras centrales ampliadas que acá se estudiara cinco ya han sido tratadas en los dos capítulos anteriores y tienen inversas que son mini L o Mini J ampliadas; En las restantes siete, sus inversos son configuraciones del mismo tipo (Barras centrales ampliadas). Todas ellas se pueden deconstruir iniciando ese proceso con el deconstructor canónico de $\mathbf{T}_{1}$ (o uno congruente) y usando además un OLL congruente al deconstructor canónico de una "Cruz Ampliada" a precisar en su momento.

Comentario Las Barras centrales ampliadas de más fácil construcción se diseñan iniciando ese proceso con el constructor de una "Cruz Ampliada" y operándolo luego con el constructor de $T_{1}$ :

"Cruz Ampliada" + $\mathrm{T}_{\mathbf{1}} \longrightarrow$ Barra Central Ampliada

\subsection{Cinco primeras "Barras Centrales Ampliadas"}

11.1.1 Z $=\mathrm{Pez}_{\mathrm{L}}+\mathrm{U} 2+\mathrm{T}_{1}$ : “La Z"40

( Inversa de $\mathrm{P}_{2}, 9.2 .7$ ).
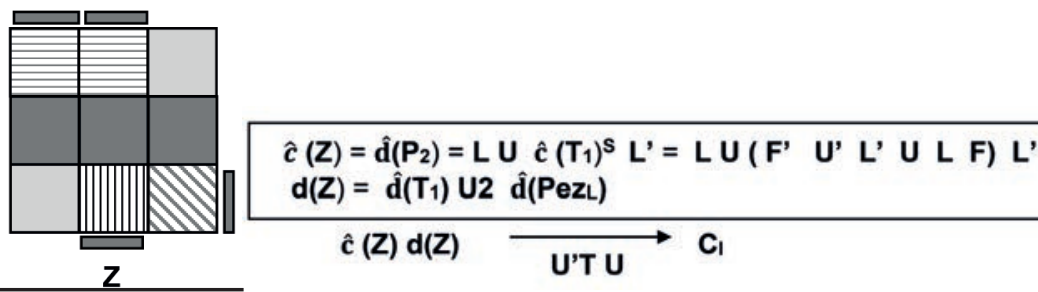

U'T U

40 Se le da este nombre a la configuración pues cuando se rota la capa superior $90^{\circ}$ sentido horario o antihorario se observa una $\mathrm{Z}$ en la parte superior de dicha capa. 
donde $\mathrm{d}\left(\mathrm{T}_{1}\right)=$ F R U R' U' F', 1.6.1;

$\widehat{\mathrm{d}}\left(\mathrm{Pez}_{\mathrm{L}}\right)=\mathrm{L}^{\prime} \mathrm{U}^{\prime} \mathrm{L} \mathrm{U}^{\prime} \quad$ L' U2 L, 8.2.2; y

$T=R U R^{\prime} U^{\prime} R^{\prime} \quad$ FR2 $\quad U^{\prime} R^{\prime} U^{\prime}$ RU R' R', 6.4.2.

11.1.2 S = Camaleón + U + T : “La S"41

(Simétrica de “Z” e Inversa de $\mathrm{q}_{2}, 10.1 .6$ )
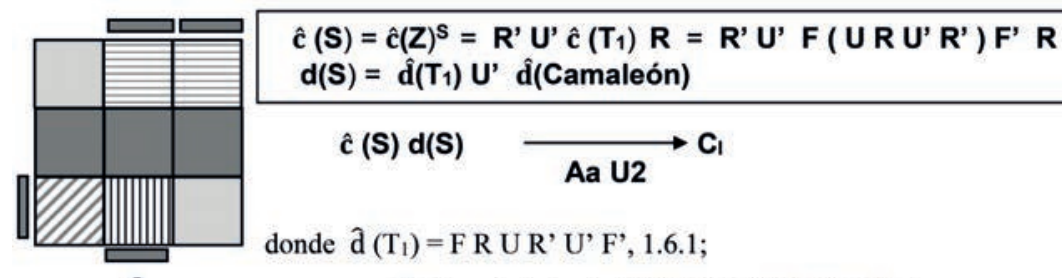

donde $\mathrm{d}\left(\mathrm{T}_{1}\right)=\mathrm{F}$ R U R' U' F', 1.6.1;

S

d (Camaleón) $=$ (r U R' U') (r' F R F'), 7.6.1; y

$$
\mathrm{Aa}=\mathrm{R}^{\prime} \mathrm{F}^{\prime} \mathrm{B}^{\prime} \quad \mathrm{B} 2 \quad \mathrm{R} \mathrm{F}^{\prime} \mathrm{R} \quad \mathrm{R} 2 \mathrm{~B} 2 \mathrm{R} 2,2.6 .1 .2 . \quad \mathrm{C}
$$

11.1.3 $\mathrm{L}_{1}=\mathrm{L}(0,2,1,2)=\mathrm{Cruz}_{2}+\mathrm{U}+\mathrm{T}_{1}+\mathrm{U}^{\prime}$ :

La $1^{\text {a }} \mathbf{L}$

(Inversa de $\mathbf{Y}_{\mathrm{I}}$, 9.3.6)

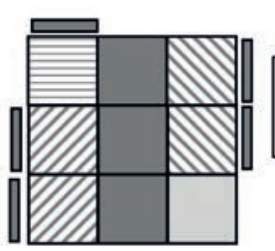

$\mathbf{L}_{1}$
$\hat{c}\left(L_{1}\right)=d\left(Y_{1}\right)=R^{\prime} U^{\prime}(R$ F R' F') U (F R F')

$d\left(L_{1}\right)=U \hat{d}\left(T_{1}\right) U^{\prime} \hat{d}\left(C_{r u z}\right)$

$\hat{c}\left(L_{1}\right) d\left(L_{1}\right) \underset{R_{D} U^{\prime}}{\longrightarrow} C_{1}$

donde $\mathrm{d}\left(\mathrm{T}_{1}\right)=\mathrm{F}$ R U R' U' F', 1.6.1;

d $\left(\right.$ Cruz $\left._{2}\right)=$ R U2 R'2 U' R2 U' R2 U2 R, 7.4.1; y

$\mathrm{R}_{\mathrm{D}}=\mathrm{R}^{\prime} \mathrm{U} 2 \mathrm{R}$ U2 R' FRU R' U'R' F' R2 U', 6.1.1.

41 Se le da este nombre a la configuración pues cuando se rota la capa superior $90^{\circ}$ sentido horario o antihorario se observa una $\mathrm{S}$ en la parte superior de dicha capa. 


\subsection{4 $\mathrm{J}_{1}=\mathrm{J}(\mathbf{0}, 2,1,2)=\mathrm{Cruz}_{2}+\mathrm{U}^{\prime}+\mathrm{T}_{1}+\mathrm{U}^{\prime}$ :}

\section{La $1^{\mathrm{a}} \mathrm{J}$}

(Simétrica de la $1^{\mathrm{a}}$ "L" e Inversa de $\mathrm{Y}_{\mathrm{D}}, 10.2 .6$ ).

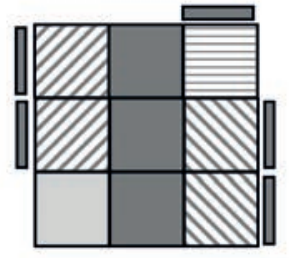

$\mathbf{J}_{1}$

$$
\begin{aligned}
& \hat{c}\left(J_{1}\right)=\hat{c}\left(L_{1}\right)^{s}=L U\left(L^{\prime} F^{\prime} L F\right) \quad U^{\prime} \quad\left(F^{\prime} L^{\prime} F\right) \\
& d\left(J_{1}\right)=U \hat{d}\left(T_{1}\right) U \hat{d}\left(C r u z_{2}\right)
\end{aligned}
$$

$$
\hat{c}\left(\mathrm{~J}_{1}\right) \mathrm{d}\left(\mathrm{J}_{1}\right) \underset{\mathrm{U}^{\prime} \mathrm{J}_{\mathrm{D}}}{\longrightarrow} \mathrm{C}_{1}
$$

donde $\widehat{\mathrm{d}}\left(\mathrm{T}_{1}\right)=\mathrm{F} R$ U R' U' F', 1.6.1;

d $\left(\mathrm{Cruz}_{2}\right)=\mathrm{R}$ U2 R'2 U' R2 U' R2 U2 R, 7.4.1; y

$J_{D}=B$ U' B L2 F' D F' D' F2 L2 B2, 4.4.1.

11.1.5 $\mathrm{I}_{3}=\mathrm{I}(1,2,1,2)=$ Camaleón $+\mathrm{T}_{1}$ :

La Tercera $\mathrm{I}^{\mathbf{4 2}}$

(Inversa de $\mathrm{mJ}_{3}, 9.4 .5$ )

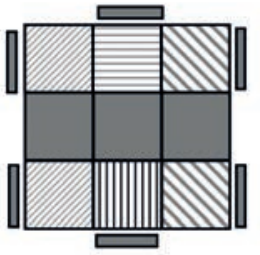

$I_{3}$
$\hat{c}\left(I_{3}\right)=\hat{d}\left(m_{3}\right)=(r$ U R U' r') F(R' URU' R' ) F' $\mathrm{d}\left(\mathrm{l}_{3}\right)=\mathrm{d}\left(\mathrm{T}_{1}\right) \mathrm{d}($ Camaleón)

$r=$ MR: movimiento parecido al movimiento $R$ solo que con " $r$ " se rotan simultáneamente las capas $R$ y su intermedia contigua $\mathrm{M}$ (sentido horario $90^{\circ}$ )

c $\left(l_{3}\right) d\left(l_{3}\right)$

E

donde $\mathrm{d}\left(\mathrm{T}_{1}\right)=\mathrm{F}$ R U R' U' F', 1.6.1;

$\widehat{\mathrm{d}}($ Camaleón $)=(\mathrm{r}$ U R' U') (r' F R F'), 7.6.1; y

$E=\left(R^{\prime} U L^{\prime} \quad D 2 \quad L U^{\prime} R\right)\left(L^{\prime} U R^{\prime}\right.$ D2 R U' L), 2.9.2.

Síntesis tabular OLL “Canónica” $(11.1 .1 \rightarrow 11.1 .5)$

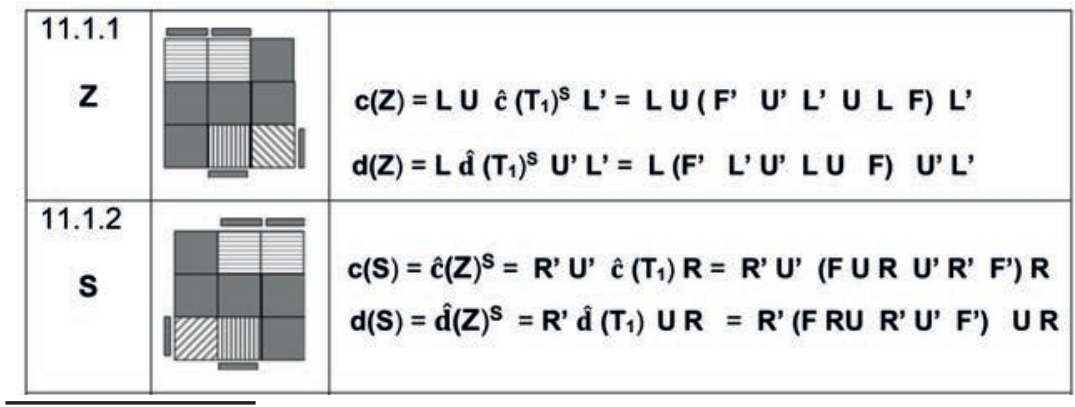

42 Existen otras dos “I” a estudiar más adelante en este capítulo. Pero hemos mencionado primero la $\mathrm{I}_{3}$ pues apareció ya en el capítulo 9 como inversa de la $\mathrm{mJ}_{3}$. 


\begin{tabular}{|c|c|}
\hline $\begin{array}{r}11.1 .4 \\
J_{1}\end{array}$ & $\begin{array}{l}c\left(J_{1}\right)=\hat{c}\left(L_{1}\right)^{S}=L U^{\prime}\left(L^{\prime} F^{\prime} L F\right) \quad U^{\prime} \text { (F' L'F) } \\
d\left(J_{1}\right)=\hat{d}\left(L_{1}\right)^{s}=\left(F^{\prime} L F\right) U\left(F^{\prime} L^{\prime} F(L) U^{\prime} L^{\prime}\right.\end{array}$ \\
\hline $\begin{array}{c}11.1 .5 \\
I_{3}\end{array}$ & $\begin{array}{l}c\left(I_{3}\right)=(r \quad \text { U R U' r') F (R' URU' R') F' } \\
d\left(I_{3}\right)=F(R \text { U R' U' R) F' (r U R' U' r') }\end{array}$ \\
\hline
\end{tabular}

\subsubsection{Las inversas de esas cinco}

\begin{tabular}{|c|c|c|}
\hline & Inversa & Constructor/deconstructor de la Inversa \\
\hline $\mathbf{z}$ & $\begin{array}{c}\mathbf{P}_{\mathbf{2}} \\
(9.2 .4)\end{array}$ & $\begin{array}{l}c\left(P_{2}\right)=d(Z)=L\left(F^{\prime} L^{\prime} U^{\prime} L U F\right) U^{\prime} L^{\prime} \\
d\left(P_{2}\right)=c(Z)=L U\left(F^{\prime} U^{\prime} L^{\prime} U L F\right) L^{\prime}\end{array}$ \\
\hline $\mathbf{s}$ & $\underset{(10.1 .4)}{\mathbf{q}_{2}}$ & $\begin{array}{l}c\left(q_{2}\right)=d(S)=R^{\prime}\left(F R \cup R^{\prime} U^{\prime} F^{\prime}\right) \cup R \\
d\left(q_{2}\right)=c(S)=R^{\prime} U^{\prime}\left(F \cup R U^{\prime} R^{\prime} F^{\prime}\right) R\end{array}$ \\
\hline $\mathbf{L}_{1}$ & $\begin{array}{c}\mathbf{Y}_{\mathbf{1}} \\
(9.3 .3)\end{array}$ & $\begin{array}{l}c\left(Y_{1}\right)=d\left(L_{1}\right)=\left(F R^{\prime} F^{\prime}\right) U^{\prime}\left(F R F^{\prime} R^{\prime}\right) U R^{\prime} \\
d\left(Y_{1}\right)=c\left(L_{1}\right)=R^{\prime} U^{\prime}\left(R R^{\prime} F^{\prime}\right) U \text { (FR F') }\end{array}$ \\
\hline $\mathbf{J}_{1}$ & $\underset{(10.2 .3)}{Y_{D}}$ & $\begin{array}{l}c\left(Y_{D}\right)=d\left(J_{1}\right)=\left(F^{\prime} L F\right) U^{(F} L^{\prime} F(L) U^{\prime} L^{\prime} \\
d\left(Y_{D}\right)=c\left(J_{1}\right)=L U^{\prime}\left(L^{\prime} F^{\prime} L F\right) U^{\prime}\left(F^{\prime} L^{\prime} F\right)\end{array}$ \\
\hline $\mathbf{I}_{3}$ & $\begin{array}{c}\mathrm{mJ}_{3} \\
(9.4 .3)\end{array}$ & $\begin{array}{l}c\left(m J_{3}\right)=d\left(I_{3}\right)=F\left(R \cup R^{\prime} U^{\prime} R\right) F^{\prime}\left(r U R^{\prime} U^{\prime} r^{\prime}\right) \\
d\left(m J_{3}\right)=c\left(l_{3}\right)=\left(r U R U^{\prime} r^{\prime}\right) F\left(R^{\prime} \cup R U^{\prime} R^{\prime}\right) F^{\prime}\end{array}$ \\
\hline
\end{tabular}

La semejanza $(\sim)$ acá es con una congruente a la configuración allí nominada

\subsection{Otras cuatro "Barras Centrales Ampliadas"}

11.2.1 $\mathrm{L}_{2}=\mathrm{L}(2,1,1,1)=$ Botella $+\mathrm{T}_{1}$ :

"La Segunda L"

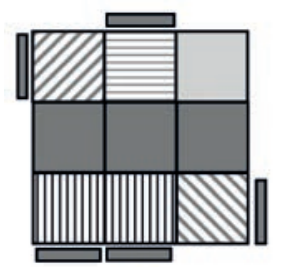

$$
\begin{aligned}
& \hat{c}\left(L_{2}\right)=(r \text { U r') (UR U' R') (r U' r') } \\
& d\left(L_{2}\right)=\hat{d}\left(T_{1}\right) \hat{d}(\text { Botella) } \\
& d\left(J_{1}\right)=\hat{d}\left(T_{1}\right) U^{\prime} \hat{d}(\text { Pajarita) } \\
& \hat{c} \mathbf{c}\left(L_{2}\right) d\left(L_{2}\right) \\
& \text { U Ab U' }
\end{aligned}
$$




$$
\begin{aligned}
& \text { donde } \hat{\mathrm{d}}\left(\mathrm{T}_{1}\right)=\mathrm{F} \text { R U R' U' F', 1.6.1; } \\
& \hat{\mathrm{d}} \text { (Botella)= R2 DR' U2 RD' R' U2 R', 7.7.2; y } \\
& \mathrm{Ab}=\mathrm{R} 2 \mathrm{~B} 2 \mathrm{R} 2 \text { R' F R' B2 R F' R, 2.6.2.2. }
\end{aligned}
$$

11.2.2 $\mathrm{J}_{2}=\mathrm{J}(2,1,1,1)=\mathrm{Pez}_{\mathrm{R}}+\mathrm{U}^{\prime}+\mathrm{T}_{1}$ :

\section{"La Segunda J"}

(Simétrica de la 2a $\mathrm{L}$ )

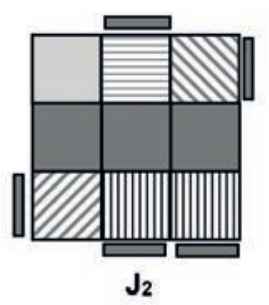

$$
\begin{aligned}
& \hat{\mathbf{c}}\left(\mathrm{J}_{2}\right)=\text { (I' U' I) (U' L' U L) (I' U I) } \\
& \mathbf{d}\left(J_{2}\right)=\hat{d}\left(T_{1}\right) U \hat{d}(\text { PezR })
\end{aligned}
$$

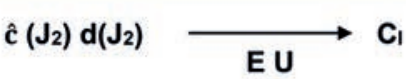

donde $\mathrm{d}\left(\mathrm{T}_{1}\right)=$ F R U R' U' F', 1.6.1;

$$
\begin{aligned}
& \hat{d}\left(P e z_{R}\right)=R \text { U R' U R U2 R', 8.1.2; y } \\
& E=\left(R^{\prime} L^{\prime} \quad\right. \text { D2 L U'R) (L'UR' D2 R U'L), 2.9.2. }
\end{aligned}
$$

E

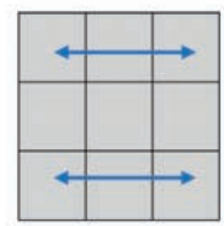

11.2.3 $C_{1}=\mathrm{C}(\mathbf{0}, \mathbf{1}, \mathbf{0}, 3)=\mathrm{Pez}_{\mathrm{L}}+\mathrm{U}+\mathrm{T}_{1}+\mathrm{U}^{\prime}$ :

"La primera C"

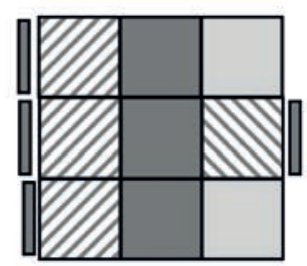

$\mathbf{C}_{1}$

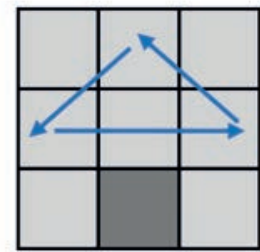

$\hat{c}\left(C_{1}\right)=L^{\prime} U^{\prime}\left(B L^{\prime} B^{\prime} L\right) U L$ $\mathbf{d}\left(C_{1}\right)=\mathbf{U} \hat{d}\left(T_{1}\right) U^{\prime} \hat{d}\left(P_{e z}\right)$

$\hat{\mathbf{c}}\left(\mathbf{C}_{1}\right) \mathrm{d}\left(\mathbf{C}_{1}\right)$

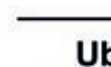

donde $\hat{\mathrm{d}}\left(\mathrm{T}_{1}\right)=$ F R U R' U' F', 1.6.1;

$$
\mathrm{d}\left(\mathrm{PeZ}_{\mathrm{L}}\right)=\mathrm{L}^{\prime} \mathrm{U}^{\prime} \mathrm{L} \mathrm{U}^{\prime} \mathrm{L}^{\prime} \mathrm{U} 2 \mathrm{~L}, 8.2 .2 ; \mathrm{y}
$$

$\mathrm{Ub}=\mathrm{M} 2 \mathrm{U}$ M $\quad \mathrm{U} 2 \quad \mathrm{M}^{\prime} \mathrm{U} \mathrm{M} 2,2.5 .1 .2 . \quad \square$ 


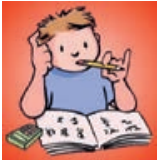

Actividad para el lector: $\mathrm{C}_{1}{ }^{\mathrm{S}} \sim \mathrm{C}_{1} \mathrm{U} 2$. Comprobar que el simétrico de $\mathrm{C}_{1}$ es semejante a la configuración $\mathrm{C}_{1}$ U2 (congruente con $\mathrm{C}_{1}$ ):

U2 d̂ (C $C_{1} s^{2} \underset{U^{\prime} U b U '}{\longrightarrow} \quad C_{l} \quad y$

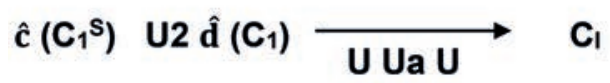

donde $\hat{d}\left(C_{1}\right)=L^{\prime} U^{\prime}$ ( L' B L B') UL $\hat{c}\left(C_{1}{ }^{S}\right)=R$ U (B' R B R') U' R' $\widehat{d}\left(C_{1}{ }^{S}\right)=R U\left(B^{\prime} R^{\prime} B\right) U^{\prime}$

11.2.4 $\mathrm{C}_{2}=\mathrm{C}(1,1,1,1)=$ Pajarita $+\mathrm{U}^{\prime}+\mathrm{T}_{1}+\mathrm{U}^{\prime}$ :

"La segunda $C$ "

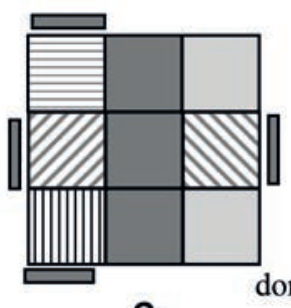

$\mathrm{C}_{2}$

$$
\begin{aligned}
& \hat{c}\left(C_{2}\right)=L^{\prime}(R \text { B' R' B) L (U B U' B') } \\
& d\left(C_{2}\right)=U \hat{d}\left(T_{1}\right) U \text { d }(\text { Pajarita) }
\end{aligned}
$$

$$
\hat{c}\left(C_{2}\right) d\left(C_{2}\right) \underset{\text { U Aa U }}{\longrightarrow} C_{1}
$$

donde $\hat{d}\left(\mathrm{~T}_{1}\right)=\mathrm{FR}$ U R' U' F', 1.6.1;

$\hat{d}$ (Pajarita) $=\left(F^{\prime} F^{\prime}\right.$ r) (U R U' r'), 7.9.1; y

$\mathrm{Aa}=\mathrm{R}^{\prime} \mathrm{FR}^{\prime} \quad \mathrm{B} 2 \quad \mathrm{R} \mathrm{F}^{\prime} \mathrm{R} \quad \mathrm{R} 2 \mathrm{~B} 2 \mathrm{R} 2,2.6 .1 .2 . \quad \square$

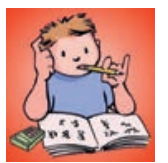

Actividad para el lector: $\mathrm{C}_{2}{ }^{\mathrm{s}} \sim \mathrm{C}_{2} \mathrm{U} 2$

Comprobar que el simétrico de $\mathrm{C}_{2}$ es semejante a la configuración $\mathrm{C}_{2} \mathbf{U} 2$ (congruente con $\mathrm{C}_{2}$ ):

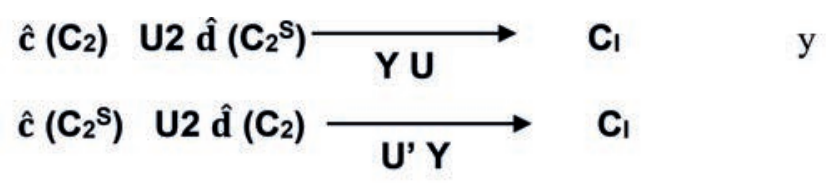

donde: $\hat{c}\left(C_{2}\right)=L^{\prime}$ (R B' R' B) L (U B U' B');

$\hat{c}\left(C_{2}{ }^{S}\right)=R\left(L^{\prime} B\right.$ L B') R' (U' B' UB);

d̂ $\left(\mathrm{C}_{2}{ }^{\mathrm{S}}\right)=\left(\mathrm{B}^{\prime} \mathrm{U}^{\prime} \mathrm{B} \mathrm{U}\right) \mathrm{R}$ (B L' B' L) R';

$\widehat{\mathrm{d}}\left(\mathrm{C}_{2}\right)=\left(\mathrm{BU} \mathrm{B}^{\prime} \mathrm{U}^{\prime}\right) \mathrm{L}^{\prime}\left(\mathrm{B}^{\prime} \mathrm{R}\right.$ B R') L; y

$\mathrm{Y}=\mathrm{F} R\left(\mathrm{U}^{\prime} \mathrm{R}^{\prime} \mathrm{U}^{\prime}\right)$ (R U R' F') (R U R'U' R'F R F'), 6.5.2 
Síntesis tabular OLL “Canónica” (11.2.1 $\rightarrow$ 11.2.4)

\begin{tabular}{|c|c|c|}
\hline $\begin{array}{r}11.2 .1 \\
\mathrm{~L}_{2}\end{array}$ & 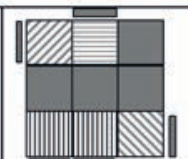 & $\begin{array}{l}c\left(L_{2}\right)=\left(r \text { U r') }\left(U R U^{\prime} R^{\prime}\right)\left(r U^{\prime} r^{\prime}\right)\right. \\
d\left(L_{2}\right)=\left(r \cup r^{\prime}\right)\left(R U R^{\prime} U^{\prime}\right)\left(r U^{\prime} r^{\prime}\right)\end{array}$ \\
\hline $\begin{array}{r}11.2 .2 \\
J_{2}\end{array}$ & & 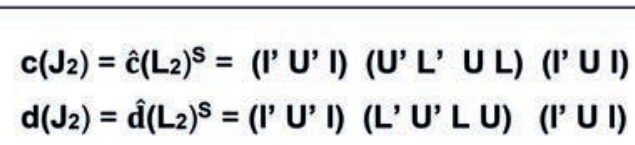 \\
\hline $\begin{array}{r}11.2 .3 \\
C_{1}\end{array}$ & & $\begin{array}{l}c\left(C_{1}\right)=L^{\prime} U^{\prime}\left(B L^{\prime} B^{\prime} L\right) U L \\
d\left(C_{1}\right)=L^{\prime} U^{\prime}\left(L^{\prime} B\left(L B^{\prime}\right) U L\right.\end{array}$ \\
\hline $\begin{array}{r}11.2 .4 \\
C_{2}\end{array}$ & & $\begin{array}{l}c\left(C_{2}\right)=L^{\prime}\left(R B^{\prime} R^{\prime} B\right) L(\text { (U B U' B') } \\
d\left(C_{2}\right)=\left(B U B^{\prime} U^{\prime}\right) L^{\prime}\left(B^{\prime} R B R^{\prime}\right) L\end{array}$ \\
\hline
\end{tabular}

\subsubsection{Las inversas de esas cuatro configuraciones}

\begin{tabular}{|c|c|c|}
\hline & Inversa & Constructor/deconstructor de la Inversa \\
\hline $\mathrm{L}_{2}$ & $\begin{array}{l}\mathbf{L}_{2}^{-1} \\
\left(\sim \mathbf{m Z}_{1}, 9.2 .3\right)\end{array}$ & $\begin{array}{l}c\left(L_{2}^{-1}\right)=d\left(L_{2}\right)=\left(r U r^{\prime}\right)\left(R U R^{\prime} U^{\prime}\right)\left(r U^{\prime} r^{\prime}\right) \\
d\left(L_{2}^{-1}\right)=c\left(L_{2}\right)=\left(r U r^{\prime}\right)\left(\text { (UR U } U^{\prime} R^{\prime}\right)\left(r U^{\prime} r^{\prime}\right)\end{array}$ \\
\hline $\mathbf{J}_{\mathbf{2}}$ & $\begin{array}{l}\mathbf{J}_{\mathbf{2}}^{-1} \\
\left(\sim \mathbf{m S}_{1}, 10.1 .3\right)\end{array}$ & $\begin{array}{l}c\left(J_{2}-1\right)=d\left(J_{2}\right)=\left(I^{\prime} U^{\prime} \mid\right)\left(L^{\prime} U^{\prime} L U\right) \quad\left(I^{\prime} U I\right) \\
d\left(J_{2}-1\right)=c\left(J_{2}\right)=\left(I^{\prime} U^{\prime} I\right)\left(U^{\prime} L^{\prime} U L\right)\left(I^{\prime} \cup I\right)\end{array}$ \\
\hline$C_{1}$ & $\begin{array}{l}\mathbf{C}_{1}-1 \\
\left(\sim \mathbf{q}_{1}, 1.9 .1\right)\end{array}$ & $\begin{array}{l}c\left(C_{1}-1\right)=d\left(C_{1}\right)=L^{\prime} U^{\prime}\left(L^{\prime} B L^{\prime} B^{\prime}\right) U L \\
d\left(C_{1}^{-1}\right)=c\left(C_{1}\right)=L^{\prime} U^{\prime}\left(B L^{\prime} B^{\prime} L\right) U L\end{array}$ \\
\hline $\mathrm{C}_{2}$ & $\begin{array}{c}\mathbf{C}_{2}^{-1} \\
\left(\sim \mathbf{C o m e t a}_{1}, 1.9 .1\right)\end{array}$ & $\begin{array}{l}c\left(C_{2}^{-1}\right)=d\left(C_{2}\right)=\left(B U B^{\prime} U^{\prime}\right) L^{\prime}\left(B^{\prime} R \text { B R' }\right) L \\
d\left(C_{2}^{-1}\right)=c\left(C_{2}\right)=L^{\prime}\left(R B^{\prime} R^{\prime} B\right) L(U B \text { U' B' })\end{array}$ \\
\hline
\end{tabular}

La semejanza $(\sim)$ acá es con una congruente a la configuración allí nominada 


\subsection{Las restantes Tres "Barras Centrales Ampliadas"}

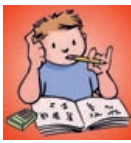

Esta sección la debe desarrollar el lector como una buena práctica y de modo similar a lo propuesto en 10.3. Además, verificar que sus "configuraciones simétricas" son "semejantes a las iniciales" (ó congruentes a estas).

11.3.1 $\mathrm{I}_{2}=\mathrm{I}_{\mathrm{V}}(0,3,0,3)=$ "La Segunda I"43

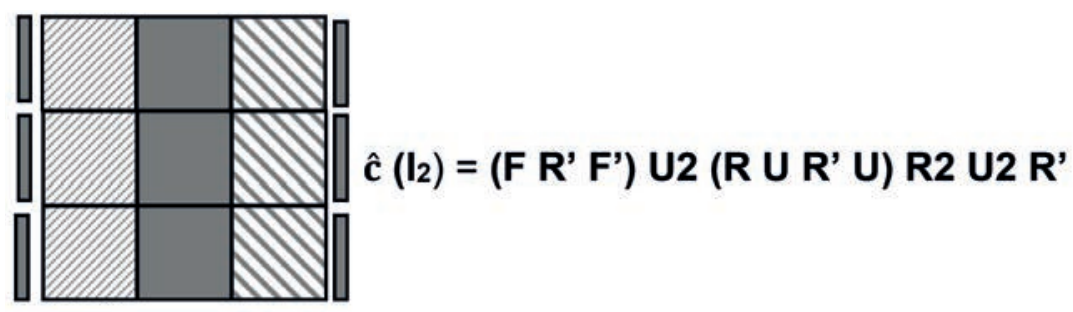

$I_{2}$

11.3.2 $\mathrm{I}_{4}=\mathrm{I}_{\mathrm{V}}(1,3,1,1)$ : "La Cuarta I"

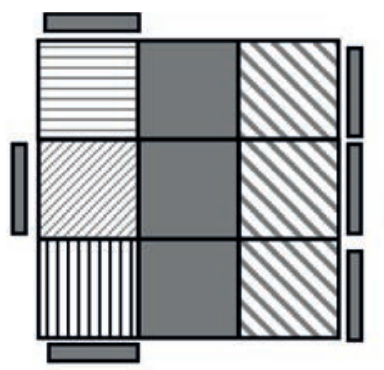

$\hat{\mathbf{c}}\left(\mathbf{I}_{4}\right)=\mathbf{R}^{\prime} \mathrm{F}^{\prime}$ (U' F U') (R U R' U R)

$\mathrm{I}_{4}$

11.3.3 La “H”

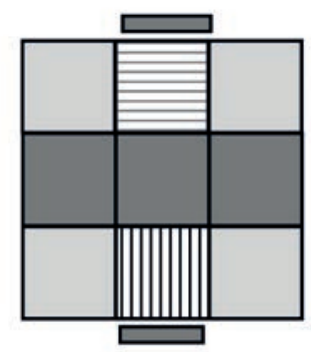

$\hat{c}(H)=M(R$ U R' U') M' (U R U' R')

H

43 Esta, por su simetría, es la I más bonita (3 bloques de sticker de mismo color y paralelos). 
Síntesis tabular OLL “Canónica” $(11.3 .1 \rightarrow 11.3 .3)$

\begin{tabular}{|c|c|}
\hline $\begin{array}{c}11.3 .1 \\
I_{2}\end{array}$ & $\begin{array}{l}c\left(I_{2}\right)=\left(F R^{\prime} F^{\prime}\right) \text { U2 (R U R' U) R2 U2 R' } \\
d\left(I_{2}\right)=R \text { U2 R2 (U' R U' R') U2 (F R F') }\end{array}$ \\
\hline $\begin{array}{c}11.3 .2 \\
I_{4}\end{array}$ & $\begin{array}{l}c\left(I_{4}\right)=R^{\prime} F^{\prime}\left(U^{\prime} F U^{\prime}\right)(R \text { U R' U R) } \\
d\left(I_{4}\right)=\left(R^{\prime} U^{\prime} R \text { U' R') (U F' U) (F R) }\right.\end{array}$ \\
\hline $\begin{array}{c}11.3 .3 \\
\mathbf{H}\end{array}$ & $\begin{array}{l}c(H)=M(R \text { U R' U') M' (U R U' R') } \\
d(H)=\left(R \cup R^{\prime} U^{\prime}\right) M\left(U R U^{\prime} R^{\prime}\right) M^{\prime}\end{array}$ \\
\hline
\end{tabular}

11.3.4 Las inversas de esas tres configuraciones

\begin{tabular}{|c|c|c|}
\hline & Inversa & Constructor/deconstructor de la Inversa \\
\hline $\mathbf{I}_{2}$ & $\begin{array}{c}\mathbf{I}_{2}{ }^{-1} \\
\left(\sim \mathrm{mJ}_{1}, 5.1 .1\right)\end{array}$ & $\begin{array}{l}c\left(l_{2}^{-1}\right)=d\left(l_{2}\right)=R \text { U2 R2 (U' R U' R') U2 (F R } \\
d\left(I_{2}^{-1}\right)=c\left(I_{2}\right)=\left(F R^{\prime} F^{\prime}\right) \text { U2 (RU R' U) R2 U2 R' }\end{array}$ \\
\hline $\mathrm{I}_{4}$ & $\begin{array}{l}\mathrm{I}_{4}-1 \\
\left(\sim \mathrm{I}_{4}\right)\end{array}$ & $\begin{array}{l}c\left(1_{4}^{-1}\right)=d\left(l_{4}\right)=\left(R^{\prime} U^{\prime} R U^{\prime} R^{\prime}\right)\left(U F^{\prime} U\right)(F R) \\
d\left(l_{4}^{-1}\right)=c\left(l_{4}\right)=R^{\prime} F^{\prime}\left(U^{\prime} F U^{\prime}\right) \quad\left(R U R^{\prime} U R\right)\end{array}$ \\
\hline $\mathbf{H}$ & $\begin{array}{c}\mathbf{H}^{-1} \\
\text { ( PezALI, 9.2.1) }\end{array}$ & $\begin{array}{l}c\left(H^{-1}\right)=d(H)=\left(R \cup R^{\prime} U^{\prime}\right) \quad M\left(U R U^{\prime} R^{\prime}\right) M^{\prime} \\
d\left(H^{-1}\right)=c(H)=M\left(R U R^{\prime} U^{\prime}\right) M^{\prime}\left(U R U^{\prime} R^{\prime}\right)\end{array}$ \\
\hline
\end{tabular}

La semejanza $(\sim)$ acá es con una congruente a la configuración allí nominada

11.3.5 Práctica: Las "Barras centrales Ampliadas" a partir de Las "Cruces ampliadas"

\begin{tabular}{|c|c|}
\hline Configuración OLL & "Cruz Ampliada" + Uk + mJ $J_{1}+$ Un \\
\hline $\begin{array}{ll}11.2 .2 & \mathrm{~J}_{2}\end{array}$ & $P e z_{R}+U^{\prime}+T_{1}$ \\
\hline 11.3 .2 & $P_{e z_{R}}+U_{2}+T_{1}+U^{\prime}$ \\
\hline 11.1 .1 & $\mathrm{Pez}_{\mathrm{L}}+\mathrm{U}_{2}+\mathrm{T}_{1}$ \\
\hline 11.2 .3 & $P e z L+U+T_{1}+U^{\prime}$ \\
\hline
\end{tabular}




\begin{tabular}{|c|c|}
\hline $\begin{array}{ll}11.3 .1 & I_{2}\end{array}$ & Cruz $_{2}+T_{1}+U^{\prime}$ \\
\hline $\begin{array}{ll}11.1 .3 & \mathbf{L}_{1}\end{array}$ & $\operatorname{Cruz}_{2}+U+T_{1}+U^{\prime}$ \\
\hline $\begin{array}{ll}11.1 .4 & \mathbf{J}_{1}\end{array}$ & $\operatorname{Cruz}_{2}+U^{\prime}+T_{1}+U^{\prime}$ \\
\hline $\begin{array}{ll}11.1 .5 & I_{3} \\
\end{array}$ & Camaleón + $T_{1}$ \\
\hline $\begin{array}{ll}11.1 .2 & \mathbf{S}\end{array}$ & Camaleón $+U+T_{1}$ \\
\hline 11.2 .1 & Botella $+T_{1}$ \\
\hline 10.3 .3 & Botella + U' + $T_{1}$ \\
\hline $\begin{array}{ll}11.2 .4 & \mathrm{C}_{2} \\
\end{array}$ & Pajarita + $U^{\prime}+T_{1}+U^{\prime}$ \\
\hline
\end{tabular}

11.4 Sección Complementaria:

La "X" y su simétrica a partir de la $\mathrm{H}$

\subsubsection{Nuevos contructor/decontructor de la configuración $\mathbf{H}$}

Sean

$$
\begin{aligned}
& \mathrm{c}(\mathrm{H})=(\mathrm{MU}) 4\left(\mathrm{M}^{\prime} \mathrm{U}^{\prime}\right) 4=(\mathrm{MU} \mathrm{MU} \text { MU MU })\left(\mathrm{M}^{\prime} \mathrm{U}^{\prime}\right. \\
& \left.\mathrm{M}^{\prime} \mathrm{U}^{\prime} \mathrm{M}^{\prime} \mathrm{U}^{\prime} \mathrm{M}^{\prime} \mathrm{U}^{\prime}\right) \\
& \mathrm{d}(\mathrm{H})=\mathrm{c}(\mathrm{H})=(\mathrm{MU}) 4\left(\mathrm{M}^{\prime} \mathrm{U}^{\prime}\right)^{44}
\end{aligned}
$$

Comprobar que $\mathrm{c}(\mathrm{H})$ es un constructor de $\mathrm{H}$ y su configuración es semejante a la obtenida con $\widehat{\mathbf{c}}(\mathrm{H})$, es decir, verificar la validez de estos dos diagramas:

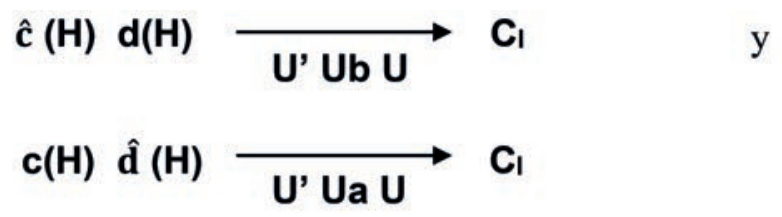

44 Este deconstructor no es propiamente el inverso de $\mathrm{c}(\mathrm{H})$, pero resulta muy práctico. Nótese que el nuevo constructor para $\mathrm{H}$ es muy parecido al constructor de la "X" tratado en la sección 10.4 del capítulo anterior, solo varían en la U final. 
donde

$$
\begin{aligned}
& \widehat{\mathrm{d}(\mathrm{H})}=(\text { RUR' U') M (URU' R') M'; } \\
& \mathrm{Ub}=\text { M2 U M } \quad \text { U2 } \quad \text { M' U M2, 2.5.2.2. y } \\
& \mathrm{Ua}=\text { M2 U' M } \quad \text { U2 } \quad \text { M' U' M2, 2.5.1.2. }
\end{aligned}
$$

$$
\begin{aligned}
& X=H+U+H+U^{\prime} \\
& X^{S}=H^{\prime}+U^{\prime}+H^{\prime}+U=X
\end{aligned}
$$

Aprovechando el ultimo constructor/deconstructor de " $H$ " generamos de una forma congruente la configuración "X", fundamental en la deconstrucción de las configuraciones puntuales (9.1.4) a tratar en el capítulo 12

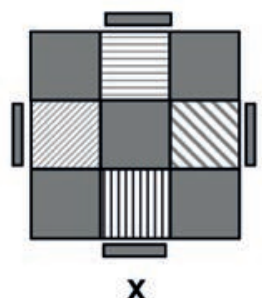

$$
\begin{aligned}
& 1^{\text {a }} \text { Forma ("canónica"): } \\
& \hat{c}(X)=\text { (MU) } 4 \text { (M'U) } 4 \\
& \hat{d}(X)=\hat{c}(X)=(M U) 4 \text { (M'U) } 4 \\
& 2^{\mathrm{a}} \text { Forma ("Nueva" } \cong \text { Canónica) } \\
& \mathbf{c}(\mathbf{X})=\mathbf{c}(\mathbf{H}) \mathbf{U} \mathbf{c}(\mathbf{H}) \mathbf{U}^{\prime} \\
& d(X)=U d(H) U^{\prime} d(H)
\end{aligned}
$$

(*) $\mathrm{c}(\mathrm{H})=(\mathrm{MU}) 4$ (M'U') 4

$$
\mathrm{d}(\mathrm{H})=\mathrm{c}(\mathrm{H})=(\mathrm{MU}) 4\left(\mathrm{M}^{\prime} \mathrm{U}^{\prime}\right) 4
$$

El lector puede comprobar la validez de estos dos diagramas:

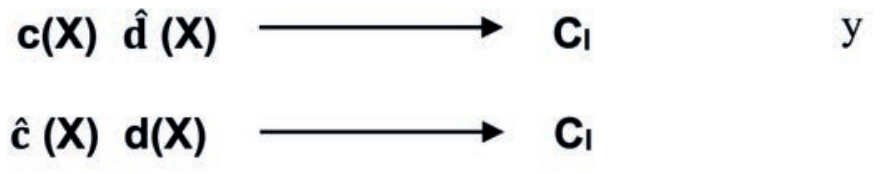




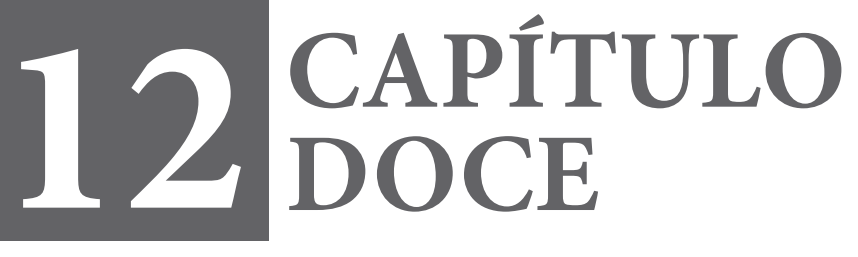




\section{Ocho configuraciones Puntuales Optimizando Tiempo en La Resolución del Cubo de Rubik: Parte $4^{\mathrm{a}}$}

Una vez conocidas e ilustradas las tres anteriores clases de configuraciones OLL vamos a realizar algo similar con las "configuraciones Puntuales"45 .Del capítulo 9 sabemos que estas configuraciones son aquellas que No son Mini J ni Mini L ampliadas ni Barras centrales Ampliadas. "Ninguna de sus aristas está bien orientada".

Todas ellas se pueden deconstruir iniciando ese proceso con el deconstructor canónico de $\mathbf{X}$ (o uno congruente) y usando además un OLL congruente al deconstructor canónico de una "Cruz Ampliada" a precisar en su momento.

Comentario Las Configuraciones Puntuales de más fácil construcción se diseñan iniciando ese proceso con el constructor de una "Cruz Ampliada" y operándolo luego con el constructor de $\mathbf{X}$ :

"Cruz Ampliada" + X $\longrightarrow$ Configuración Puntual

12.1 La "X": Base de las demás Puntuales

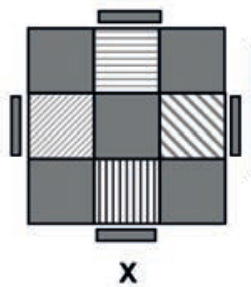

$\hat{c}(X)=(M \quad)_{4}\left(M^{\prime}\right)_{4}=(M U M U M U M U)\left(M^{\prime} U M^{\prime} M^{\prime} M^{\prime} M^{\prime} U\right)$

$\hat{d}(X)=\hat{c}(X)=(M U)_{4}\left(M^{\prime}\right)_{4}$

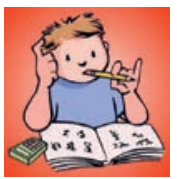

Actividad para el lector: $\mathbf{X}=\mathbf{X}^{-1}=\mathbf{X}^{\mathrm{s}}$. Usando el cubo de Rubik y partiendo de este en su estado original comprobar que los siguientes dos algoritmos dan lugar a la misma configuración " $\mathrm{X}$ ".

45 Son las configuraciones que No son Mini J ampliadas ni Mini L ampliadas ni Barras Horizontales ampliadas. 


$$
\begin{aligned}
& \mathbf{c}_{1}(\mathbf{X})=\left(\mathbf{U}^{\prime} \mathbf{M}\right)_{4}\left(\mathbf{U}^{\prime} \mathbf{M}^{\prime}\right)_{4}=\left(U^{\prime} M \quad U^{\prime} M \quad U^{\prime} M \quad U^{\prime} M\right)\left(U^{\prime} M^{\prime} U^{\prime} M^{\prime} U^{\prime} M^{\prime} U^{\prime} M^{\prime}\right)^{46} \\
& \mathbf{c}_{2}(\mathbf{X})=\hat{\mathbf{c}}(\mathbf{X})^{\mathrm{s}}=\left(\mathbf{M}^{\prime} \mathbf{U}^{\prime}\right)_{4}\left(\mathbf{M ~ U}^{\prime}\right)_{4}
\end{aligned}
$$

\subsection{El primer Punto y Su Inverso ... otro Punto}

Ninguna arista ni ningún vértice están bien orientados.

12.2.1 Punto $_{1}=$ Punto $(1,3,1,3)=$ Cruz $_{1}+\mathrm{U}+\mathrm{X}$ :

\section{"El Primer Punto"}

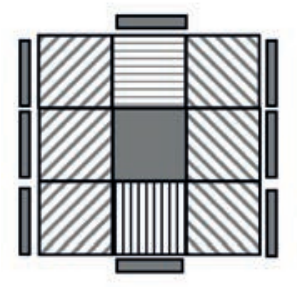

Punto1 ĉ (Punto1) = (F R' F' R) U2 (F R' F' R) R U2 R' $\hat{d}($ Punto 1$)=\hat{d}(X) U^{\prime} \hat{d}\left(\right.$ Cruz $\left._{1}\right)$

ĉ (Punto1) d(Punto 1)

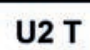

donde $\hat{d}(X)=\hat{c}(X)=(M U)_{4}\left(M^{\prime}\right)_{4}$;

$\widehat{d}\left(C_{r u z}\right)=F\left(R\right.$ U R' U') $3 F^{\prime}, 4.7 .2 ; y$

T=RU R' U' R' FR2 U'R' U' RU R'F', 6.4.2.

Actividad para el lector: Punto ${ }_{1}^{\mathrm{s}} \sim$ Punto $_{1}$ Comprobar
que el Punto
y su simétrico son Semejantes. De modo

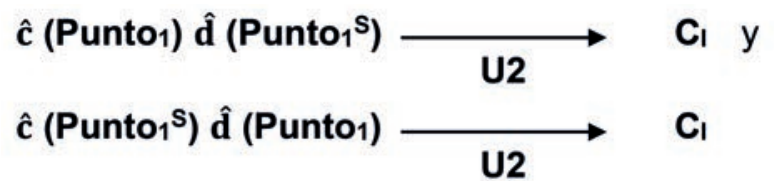

donde $\hat{c}\left(\right.$ Punto $\left._{1}^{\mathrm{S}}\right)=\left(\mathrm{F}^{\prime} \mathrm{L} F \mathrm{~L}^{\prime}\right)$ U2 ( $\left.\mathrm{F}^{\prime} \mathrm{LF} \mathrm{L}^{\prime}\right) \mathrm{L}^{\prime} \mathrm{U} 2 \mathrm{~L}$;

d̂ $\left(\right.$ Punto1 $\left.^{\text {S }}\right)=$ L' U2 L (L F' L' F) U2 (L F' L' F); y

d̂(Punto1) $=$ R U2 R' (R' F R F') U2 (R' F R F')

46 Este constructor es el verdadero inverso del constructor canónico de $\mathbf{X}$. 
12.2.2 El Otro Punto: El inverso canónico del Punto Punto $_{2}=\operatorname{Punto}(2,3,2,1)=\mathrm{Cruz}_{2}+\mathrm{U} 2+\mathrm{X}$ : "El segundo Punto"

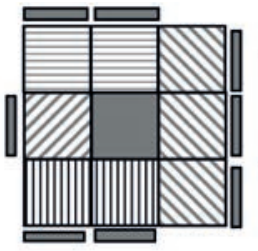

$\hat{\mathbf{c}}$ (Punto2) $=\mathrm{d}\left(\right.$ Punto $\left._{1}\right)=$ R U2 R' (R' F R F') U2 (R' F R F') $\mathbf{d}($ Punto2 $)=\hat{d}(X)$ U2 $\hat{d}\left(\right.$ Cruzz $\left._{2}\right)$

$\hat{c}$ (Punto2) d(Punto2) $\underset{\text { Ga U2 }}{\longrightarrow} \mathrm{C}_{1}$

Punto2

donde $\hat{d}(X)=\hat{c}(X)=\left(M^{\prime}\right)_{4}\left(M^{\prime} U\right)_{4}$;

d $\left(\mathrm{Cruz}_{2}\right)=$ R U2 R'2 U' R2 U' R2 U2 R, 7.4.1; y

$\mathrm{Ga}=\mathrm{L}^{\prime}$ U' L F2 D R' U R U'R D' F2, 5.3.3.

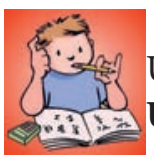

Actividad para el lector: Punto $_{2}{ }^{\mathrm{S}} \cong$ Punto $_{2}$

Usando el cubo de Rubik comprobar que Punto $_{2}{ }^{\mathrm{S}}=$ Punto $_{2}$ U2

con $\hat{\mathbf{c}}\left(\right.$ Punto $\left._{2}{ }^{S}\right)=$ L' U2 L (L F' L' F) U2 (L F' L' F). Y luego verificar:

donde

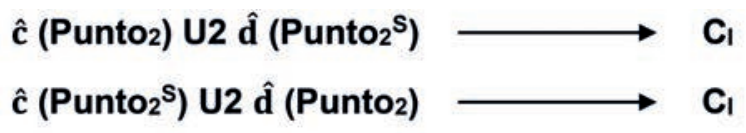

d $\left(\right.$ Punto2 $\left.^{\mathrm{S}}\right)=\left(\mathrm{F}^{\prime}\right.$ L F L') U2 (F' LF L') L' U2 L; y

$\hat{\mathrm{d}}\left(\right.$ Punto2 $\left._{2}\right)=\hat{\mathrm{c}}\left(\right.$ Punto $\left._{1}\right)=\left(\mathrm{F}^{\prime} \mathrm{F}^{\prime} \mathrm{R}\right) \mathrm{U} 2$ (F R' F' R) R U2 R'

12.3 $\mathrm{D}_{\mathrm{NP} 1}$ : La Primera Diagonal No Principal y su inversa $\mathrm{V}_{1}$ Solo dos vértices orientados.

12.3.1 $\mathrm{D}_{\mathrm{NP1}}=\mathrm{D}_{\mathrm{NP}}(1,1,2,2)=$ Pajarita $+\mathrm{U} 2+\mathrm{X}$ :

“ $1{ }^{a}$ Diagonal NO Principal"

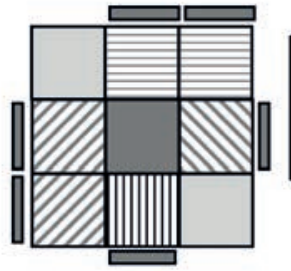

$\hat{c}\left(D_{N P 1}\right)=\left(F R^{\prime} F^{\prime} R\right.$ U2 F R' F' R) (U' R U' R')

$d\left(D_{N P 1}\right)=\hat{d}(X)$ U2 $\hat{d}\left(P_{\text {ajarita }}\right)$

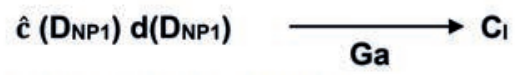

DNP1 $^{\text {donde }} \hat{\mathrm{d}}(\mathrm{X})=\hat{\mathrm{c}}(\mathrm{X})=(\mathrm{M} \mathrm{U})_{4}\left(\mathrm{M}^{\prime} \mathrm{U}\right)_{4}$; 
donde $\hat{\mathrm{d}}(\mathrm{X})=\hat{\mathrm{c}}(\mathrm{X})=(\mathrm{M} \mathrm{U})_{4}\left(\mathrm{M}^{\prime} \mathrm{U}\right)_{4}$;

$\hat{\mathrm{d}}($ Pajarita $)=\left(\mathrm{F}^{\prime} \mathrm{F}^{\prime}\right.$ r) $\left(\mathrm{U}^{\mathrm{R}} \mathrm{U}^{\prime} \mathrm{r}^{\prime}\right)$, 7.9.1; y

$\mathrm{Ga}=\mathrm{L}^{\prime} \mathrm{U}^{\prime} \mathrm{L} \quad \mathrm{F} 2$ D R' U R U'R D' F2, $5.3 .3 \quad \mathrm{a}$

Actividad para el lector: La primera diagonal principal o Simétrica de la primera diagonal no principal. $\mathrm{D}_{\mathrm{P1}}=\mathrm{D}_{\mathrm{NP1}}{ }^{\mathrm{s}}=\mathrm{D}_{\mathrm{P}}(1,2,2,1)$. Usando el cubo de Rubik comprobar que $\mathbf{D}_{\mathrm{P}_{1}} \sim \mathrm{D}_{\mathrm{NP1}} \mathrm{U}$ con

$$
\hat{c}\left(D_{P 1}\right)=\hat{c}\left(D_{N P 1}{ }^{S}\right)=\left(F^{\prime}\right. \text { L F L' U2 F' L F L') (U L' U L). }
$$

$\mathrm{Y}$ luego verificar

$$
\begin{aligned}
& \hat{c} \text { (DP1) U' d̂ (DNP1) } \underset{\text { U T }}{\longrightarrow} C_{1} \\
& \hat{c}\left(D_{N P 1}\right) \cup \hat{d}\left(D_{P 1}\right) \underset{U T}{\longrightarrow} c_{।}
\end{aligned}
$$

donde:

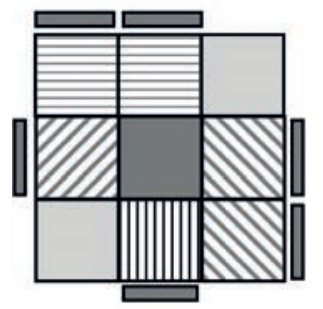

Dp1
$\widehat{d}\left(D_{N P 1}\right)=(R$ U R' U) (R' F R F' U2 R' F R F')

$\hat{c}\left(D_{N P 1}\right)=(F$ R' F' R U2 F R' F' R) (U' R U' R')

$\hat{\mathrm{d}}\left(\mathrm{D}_{\mathrm{PI}}\right)=\left(\mathrm{L}^{\prime} \mathrm{U}^{\prime} \mathrm{L} \mathrm{U}^{\prime}\right)\left(\mathrm{L} \mathrm{F}^{\prime} \mathrm{L}^{\prime} \mathrm{F}\right.$ U2 L F' L'F)

$T=R U R^{\prime} U^{\prime} R^{\prime} \quad F R 2 \quad U^{\prime} R^{\prime} U^{\prime} R U$ R'F', 6.4.

12.3.2 $\mathrm{V}_{1}$ : La primera V o Inversa de la Primera Diagonal NO Principal

$$
\mathrm{V}_{1}=\mathrm{V}(3,1,1,1)=\text { Botella }+\mathrm{X}: \text { "La Primera V" }
$$

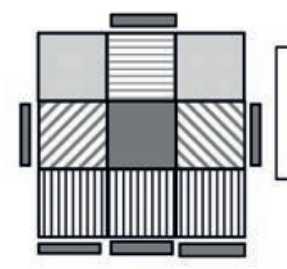

$$
\hat{c}\left(\mathbf{V}_{1}\right)=\hat{d}\left(D_{N P 1}\right)=(R \quad \text { U R' U) ( R' F R F' U2 R' F R F') }
$$$$
d\left(V_{1}\right)=\hat{d}(X) \hat{d}(\text { Botella })
$$

$\mathbf{V}_{1}$

$$
\hat{c}\left(V_{1}\right) d\left(V_{1}\right) \underset{U^{\prime} U b U^{\prime}}{\longrightarrow} c_{1}
$$

donde $\hat{\mathrm{d}}(\mathrm{X})=\hat{\mathrm{c}}(\mathrm{X})=(\mathrm{M} \mathrm{U})_{4}\left(\mathrm{M}^{\prime} \mathrm{U}\right)_{4}$;

$\mathrm{d}($ Botella $)=\mathrm{R} 2 \quad$ DR' U2 RD' R' U2 R', 7.7.2; y

$\mathrm{Ub}=\mathrm{M} 2 \mathrm{U}$ M U2 M' U M2, 2.5.2.2 


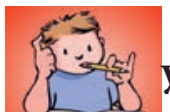

Actividad para el lector: La Simétrica de la primera V y su semejanza con ésta. Usando el cubo de Rubik $\mathrm{V}_{1}^{\mathrm{s}} \sim \mathrm{V}_{1}$ con

$$
\hat{c}\left(V_{1}{ }^{S}\right)=\left(L^{\prime} U^{\prime} L^{\prime} U^{\prime}\right) \text { (L F' L' F U2 L F' L' F) }
$$

Y luego verificar

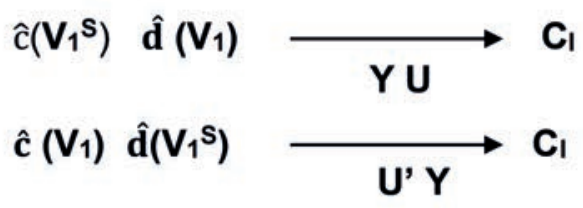

donde

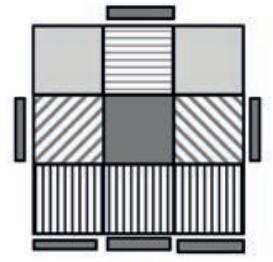

$\hat{d}\left(V_{1}\right)=\hat{c}\left(D_{N P 1}\right)=(F$ R' F' R U2 F R' F' $R)\left(U^{\prime} R U^{\prime} R^{\prime}\right)$ $\hat{c}\left(V_{1}\right)=(R$ U R' U) (R' F R F' U2 R' F R F') $\widehat{d}\left(V_{1}{ }^{S}\right)=\left(F^{\prime} L F\right.$ L'U2 F' LF L') (U L'U L) $\mathrm{Y}=\mathrm{F}$ R (U' R' U') (R U R' F') (R U R'U' R'F R F'), 5.11.3

$\mathbf{V}_{1} \mathbf{s}$

12.4 Síntesis tabular OLL “Canónica” $(\mathbf{1 2 . 1} \rightarrow \mathbf{1 2 . 3})$

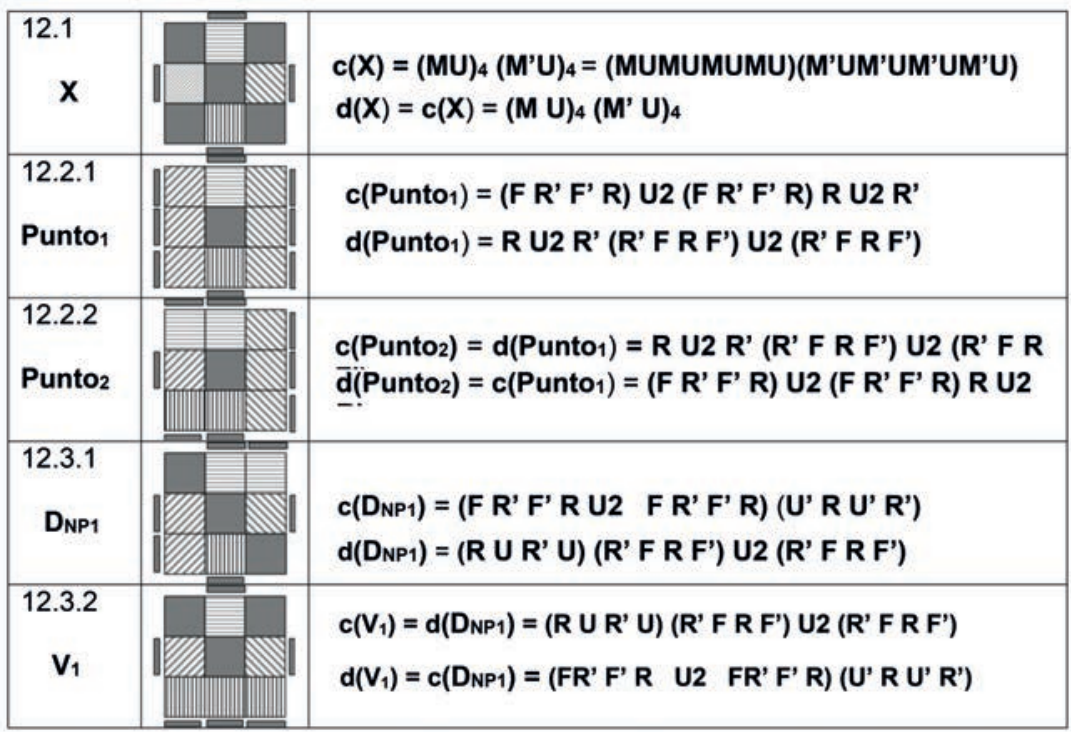


Las inversas de esas cinco configuraciones

\begin{tabular}{|c|c|c|}
\hline & Inversa & Constructor/deconstructor de la Inversa \\
\hline $\mathbf{x}$ & $\mathbf{x}$ & $c(X)=d(X)=(M)_{4}\left(M^{\prime}\right)_{4}$ \\
\hline Punto 1 & Punto 2 & $\begin{array}{l}c\left(\text { Punto }_{2}\right)=d\left(\text { Punto }_{1}\right)=\text { R U2 R' (R' F R F') U2 (R' F R F') } \\
d\left(\text { Punto }_{2}\right)=c\left(\text { Punto }_{1}\right)=(\text { F R' F' R) U2 (F R' F' R) R U2 R' }\end{array}$ \\
\hline Punto 2 & Punto 1 & $\begin{array}{l}c\left(\text { Punto }_{1}\right)=\mathrm{d}\left(\text { Punto }_{2}\right)=(\text { F R' F' R) U2 (F R' F' R) R U2 R' } \\
\mathrm{d}\left(\text { Punto }_{1}\right)=\mathrm{c}\left(\text { Punto }_{2}\right)=\text { R U2 R' (R' F R F') U2 (R' F R F') }\end{array}$ \\
\hline D $_{\text {NP1 }}$ & $\mathbf{V}_{\mathbf{1}}$ & $\begin{array}{l}c\left(V_{1}\right)=d\left(D_{\left.N P_{1}\right)}\right)=\left(R U R^{\prime} U_{)}\left(R^{\prime} F R F^{\prime}\right) U_{2}\left(R^{\prime} F R F^{\prime}\right)\right. \\
d\left(V_{1}\right)=c\left(D_{\left.N P_{1}\right)}\right)=\left(F R^{\prime} F^{\prime} R \text { U2 FR' } F^{\prime} R\right)\left(U^{\prime} R U^{\prime} R^{\prime}\right)\end{array}$ \\
\hline $\mathbf{V}_{\mathbf{1}}$ & DNP1 $_{\text {N }}$ & 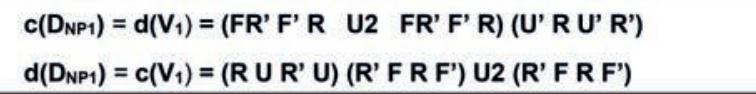 \\
\hline
\end{tabular}

\subsection{SMDIP $_{1}$}

La primera semidiagonal inferior de la Principal y su inversa... una semidiagonal superior Un solo vértice orientado.

$$
\text { 12.5.1 } \operatorname{SMDIP}_{1}=\operatorname{SMDP}(1,2,2,2)=\mathrm{Pez}_{\mathrm{L}}+\mathrm{U}+\mathrm{X} \text { : }
$$

La primera semidiagonal inferior de la Principal

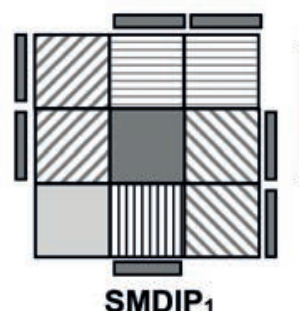

$$
\begin{aligned}
\hat{c}\left(S^{\prime} M I_{1}\right) & =c\left(T_{1}\right) U d\left(T_{1}\right) \\
& =F\left(U R U^{\prime} R^{\prime}\right) F^{\prime} U F\left(R U R^{\prime} U^{\prime}\right) F^{\prime} \\
d\left(S^{\prime} P_{1}\right) & =\hat{d}(X) U^{\prime} d \hat{d}\left(P^{\prime} \text { ezL }\right)
\end{aligned}
$$

$$
\hat{c}\left(S_{M D I P}\right) d\left(S^{\prime} M I_{1}\right) \underset{\text { U' Ub U }}{\longrightarrow}
$$

$$
\begin{aligned}
\text { donde } \hat{\mathrm{d}}(\mathrm{X})= & \hat{\mathrm{c}}(\mathrm{X})=(\mathrm{M} \mathrm{U})_{4}\left(\mathrm{M}^{\prime} \mathrm{U}\right)_{4} ; \\
& \hat{\mathrm{d}}(\mathrm{Pez} \mathrm{L})=\left(\mathrm{L} \mathrm{U}^{\prime} \mathrm{U}^{\prime} \mathrm{L} \mathrm{U}^{\prime}\right)\left(\mathrm{L}^{\prime} \mathrm{U} 2 \mathrm{~L}\right), 8.2 .2 ; \mathrm{y} \\
& \mathrm{Ub}=\text { M2 U M U2 } \text { M' U M2, 2.5.2.2. }^{\prime}
\end{aligned}
$$


12.5.2 $\mathrm{SMDIP}_{1}^{-1}=\mathrm{Pez}_{\mathrm{R}}+\mathrm{U}+\mathrm{X}$ :

La Inversa de la $1^{\text {a }}$ semidiagonal inferior de la Principal
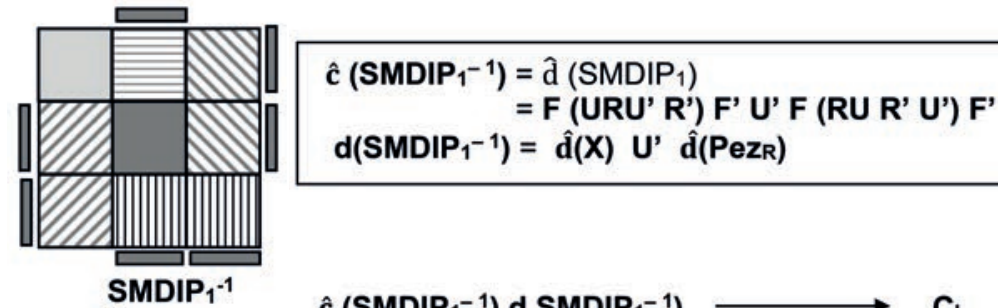

donde $\hat{d}(X)=\hat{c}(X)=(M U)_{4}\left(M^{\prime} U\right)_{4}$;

$\widehat{d}\left(P_{R}\right)=R U R ' U \quad R U 2 R^{\prime}, 8.1 .2 ; y$

$\mathrm{H}=(\mathrm{M} 2 \mathrm{U} \mathrm{M} 2) \mathrm{U} 2$ (M2 U M2), 2.7.2.

Actividad para el lector: $\operatorname{SMDIP}_{1}^{\mathrm{S}} \sim \mathrm{SMDIP}_{1}^{-1}$

Usando el cubo de Rubik comprobar que SMDIP ${ }_{1}^{-1} \sim \operatorname{SMDIP}_{1}{ }_{1}$

$\mathrm{U} 2$ con

$$
\widehat{\mathbf{C}}\left(\mathrm{SMDIP}_{1}^{\mathrm{S}}\right)=\mathrm{c}\left(\mathrm{T}_{1}^{\mathrm{S}}\right) \mathrm{U}^{\prime} \mathrm{d}\left(\mathrm{T}_{1}^{\mathrm{S}}\right)
$$

Y luego verificar

$$
\begin{aligned}
& \hat{c}\left(S_{M D I P}{ }^{S}\right) \text { U2 } \hat{d}\left(S_{M D I P}{ }^{-1}\right) \underset{\text { U2 Ub }}{\longrightarrow} C_{\text {I }} \\
& \hat{c}\left(S_{M D I P}{ }^{-1}\right) \text { U2 } \hat{d}\left(S_{M D I P} s^{s}\right) \underset{\text { Ua U2 }}{\longrightarrow} C_{1}
\end{aligned}
$$

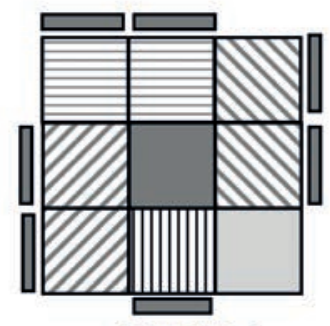

SMDIP $_{1}$ s
$\hat{\mathrm{d}}\left(\mathrm{SMDIP}_{1}^{-1}\right)=\hat{\mathrm{c}}\left(\mathrm{T}_{1}\right) \mathrm{U} \hat{\mathrm{d}}\left(\mathrm{T}_{1}\right)$

$\hat{\mathrm{c}}\left(\mathrm{SMDIP}_{1}^{-1}\right)=\hat{\mathrm{c}}\left(\mathrm{T}_{1}\right) \mathrm{U}^{\prime} \hat{\mathrm{d}}\left(\mathrm{T}_{1}\right)$

$\hat{\mathrm{d}}\left(\mathrm{SMDIP}_{1}^{\mathrm{S}}\right)=\hat{\mathrm{c}}\left(\mathrm{T}_{1}^{\mathrm{S}}\right) \mathrm{Ud}\left(\mathrm{T}_{1}^{\mathrm{S}}\right)$

$\mathrm{Ub}=\mathrm{M} 2 \mathrm{U} M \quad \mathrm{U} 2 \quad \mathrm{M}^{\prime} \mathrm{U} \mathrm{M} 2,2.5 .2 .2$

$\mathrm{Ua}=\mathrm{M} 2 \mathrm{U}^{\prime} \mathrm{M} \quad \mathrm{U} 2 \quad \mathrm{M}^{\prime} \mathrm{U}^{\prime} \mathrm{M} 2,2.5 .1 .2$ 


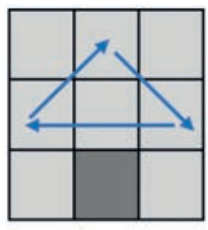

Ua

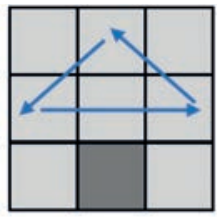

Ub

\section{6 $\mathrm{SMDIP}_{2}$ :}

La segunda semidiagonal inferior de la Principal y su inversa... una semidiagonal superior

\subsection{1 $\mathrm{SMDIP}_{2}=\operatorname{SMDP}(2,2,2,1)=\mathrm{Pez}_{\mathrm{R}}+\mathrm{X}$ :}

La segunda semidiagonal inferior de la Principal

Esta segunda semidiagonal se construye dándole un giro de $90^{\circ}$ sentido antihorario (U') a la configuración inversa de la primera semidiagonal: $\hat{\mathbf{c}}\left(\mathbf{S M D I P}_{2}\right)=\hat{\mathbf{d}}\left(\mathbf{S M D I P}_{2}\right) \mathbf{U}$,

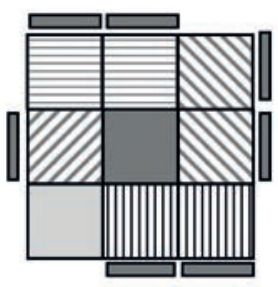

$\mathrm{SMDIP}_{2}$

$$
\begin{aligned}
& \hat{c}\left(S^{\prime} M P_{2}\right)=c\left(T_{1}\right) U^{\prime} d\left(T_{1}\right) \\
& \text { = F (URU' R') F' U' F (RU R' U') F' U' } \\
& \mathbf{d}\left(\text { SMDIP }_{2}\right)=\hat{d}(X) \quad \hat{d}(\text { PezR })
\end{aligned}
$$

$\hat{\mathbf{c}}\left(\mathrm{SMDIP}_{2}\right) \mathrm{d}\left(\mathrm{SMDIP}_{2}\right)$

H U2

$$
\begin{aligned}
& \text { donde } \hat{\mathrm{d}}(\mathrm{X})=\hat{\mathrm{c}}(\mathrm{X})=(\mathrm{M} U)_{4}\left(\mathrm{M}^{\prime} \mathrm{U}\right)_{4} ; \\
& \hat{\mathrm{d}}(\text { PezR })=\text { R U R' U R U2 R', 8.1.2; y } \\
& \mathrm{H}=(\mathrm{M} 2 \mathrm{U} \text { M2) U2 (M2 U M2), 2.7.2. }
\end{aligned}
$$

12.6.2 $\mathrm{SMDIP}_{2}^{-1}=\mathrm{Pez}_{\mathrm{L}}+\mathrm{U}+\mathrm{X}$ :

La Inversa de la segunda semidiagonal inferior de la Principal

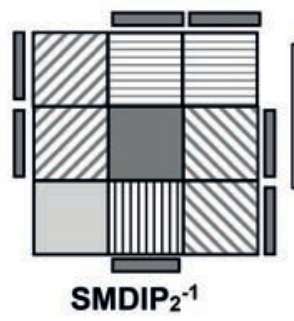

$$
\begin{aligned}
& \hat{\mathbf{c}}\left(\mathrm{SMDIP}_{2}{ }^{-1}\right)=\hat{\mathrm{d}}\left(\mathrm{SMDIP}_{2}\right) \\
& \text { = U F (URU' R') F' U F (RU R' U') F' } \\
& d\left(\text { SMDIP }_{2}{ }^{-1}\right)=\hat{d}(X) U^{\prime} \hat{d}\left(\text { PezL }^{\prime}\right)
\end{aligned}
$$

$\left.\hat{c}\left(\mathrm{SMDIP}_{2}^{-1}\right) \mathrm{d} \mathrm{SMDIP}^{-1}\right) \underset{\text { U' Ub }}{\longrightarrow} \mathrm{C}_{\mathrm{I}}$ 


$$
\begin{aligned}
& \text { donde } \hat{d}(X)=\hat{c}(X)=(M ~ U)_{4}\left(M^{\prime} \mathrm{U}\right)_{4} \text {; } \\
& \widehat{\mathrm{d}}\left(\mathrm{PeZ}_{\mathrm{L}}\right)=\left(\mathrm{L}^{\prime} \mathrm{U}^{\prime} \mathrm{L} \mathrm{U}^{\prime}\right) \text { (L' U2 L), 8.2.2; y } \\
& \mathrm{Ub}=\mathrm{M} 2 \mathrm{U} M \quad \mathrm{U} 2 \quad \mathrm{M} \text { ' UM2, 2.5.2.2. }
\end{aligned}
$$

Actividades para el lector:

1. SMDIP $_{2}^{-1} \sim$ SMDIP $_{1}$. Verificar:

1.1. $\hat{c}\left(S^{2} D^{-1} P_{2}{ }^{-1}\right) \hat{d}\left(S_{M D I P}\right)=U$

1.2. $\hat{c}\left(S_{M D I P}\right) \hat{d}\left(S_{M D I P}-1\right)=U^{\prime}$

donde

$$
\begin{array}{ll}
\hat{\mathrm{c}}\left(\mathrm{SMDIP}_{2}^{-1}\right) & =\mathrm{U} \hat{\mathrm{c}}\left(\mathrm{T}_{1}\right) \mathrm{U} \hat{\mathrm{d}}\left(\mathrm{T}_{1}\right) \\
\hat{\mathrm{d}}\left(\mathrm{SMDIP}_{1}\right) & =\hat{\mathrm{c}}\left(\mathrm{T}_{1}\right) \mathrm{U}, \hat{\mathrm{d}}\left(\mathrm{T}_{1}\right) \\
\hat{\mathrm{c}}\left(\mathrm{SMDIP}_{1}\right) & =\hat{\mathrm{c}}\left(\mathrm{T}_{1}\right) \mathrm{U} \hat{\mathrm{d}}\left(\mathrm{T}_{1}\right) \\
\hat{\mathrm{d}}\left(\mathrm{SMDIP}_{2}{ }^{-1}\right) & =\hat{\mathrm{c}}\left(\mathrm{T}_{1}\right) \mathrm{U}, \hat{\mathrm{d}}\left(\mathrm{T}_{1}\right) \mathrm{U},
\end{array}
$$

\section{SMDIP $_{2}^{\mathrm{S}} \sim \mathrm{SMDIP}_{2}^{-1}$}

Usando el cubo de Rubik comprobar que SMDIP $_{2}^{-1} \sim \operatorname{SMDIP}_{2} \mathrm{~S}^{\mathrm{S}}$ $\mathrm{U}$ con

$$
\widehat{\mathrm{c}}\left(\mathbf{S M D I P}_{2}^{\mathrm{S}}\right)=\mathbf{c}\left(\mathbf{T}_{1}{ }^{\mathrm{S}}\right) \mathbf{U} \mathbf{d}\left(\mathbf{T}_{1}{ }^{\mathrm{S}}\right) \mathbf{U}
$$

Y luego verificar

$$
\begin{aligned}
& \hat{c}\left(\mathrm{SMDIP}_{2}{ }^{\mathrm{S}}\right) \cup \text { d }\left(\mathrm{SMDIP}_{2}{ }^{-1}\right) \\
& \underset{\text { U Ub U2 }}{\longrightarrow} \mathrm{C}_{\mathrm{I}} \\
& \text { ĉ }\left(\text { SMDIP }_{2}^{-1} \text { ) U' d̂ (SMDIP } 2\right. \text { S) }
\end{aligned}
$$

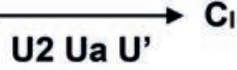

donde:

$$
\begin{aligned}
& \hat{\mathrm{d}}\left(\mathrm{SMDIP}_{2}{ }^{-1}\right)=\hat{\mathrm{c}}\left(\mathrm{T}_{1}\right) \mathrm{U}^{\prime} \hat{\mathrm{d}}\left(\mathrm{T}_{1}\right) \mathrm{U}^{\prime} \\
& \hat{\mathrm{c}}\left(\mathrm{SMDIP}_{2}^{-1}\right)=\mathrm{U} \hat{\mathrm{c}}\left(\mathrm{T}_{1}\right) \mathrm{U} \hat{\mathrm{d}}\left(\mathrm{T}_{1}\right) \\
& \widehat{d}\left(\mathrm{SMDIP}_{2}{ }^{\mathrm{S}}\right)=\mathrm{U}^{\prime} \mathrm{c}\left(\mathrm{T}_{1}^{\mathrm{S}}\right) \mathrm{U}^{\prime} \mathrm{d}\left(\mathrm{T}_{1}{ }^{\mathrm{S}}\right) \\
& \mathrm{Ub}=\mathrm{M} 2 \mathrm{U} \text { M U2 M' UM2, 2.5.2.2 } \\
& \mathrm{Ua}=\mathrm{M} 2 \mathrm{U}^{\prime} \mathrm{M} \quad \mathrm{U} 2 \quad \text { M' U' M2, 2.5.1.2 }
\end{aligned}
$$

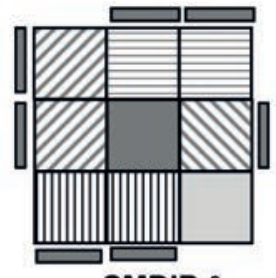

SMDIP $_{2}$ s 


\section{$12.7 \mathrm{D}_{\mathrm{NP} 2}$ :}

La Segunda Diagonal No Principal y su inversa

Dos vértices orientados.

12.7.1 $\mathrm{D}_{\mathrm{NP} 2}=\mathrm{D}_{\mathrm{NP}}(2,2,1,1)=$ Pajarita $+\mathrm{X}$ :

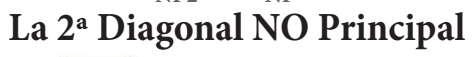

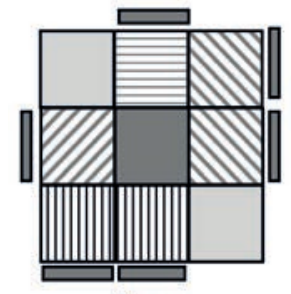

DNP2

$$
\text { c (DNP2) = M' (UR U R'U') M (R' F R F') }
$$$$
\hat{d}\left(D_{N P 2}\right)=\hat{d}(X) \quad \hat{d}(\text { Pajarita })
$$

$\hat{c}\left(D_{N P 2}\right) d\left(D_{N P 2}\right)$

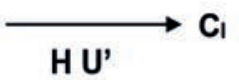

donde $\hat{d}(X)=\hat{c}(X)=(M U)_{4}\left(M^{\prime}\right)_{4}$;

$\widehat{\mathrm{d}}$ (Pajarita) $=\left(\mathrm{F}^{\prime}\right.$ ' F' r) (U R U' r'), 7.9.1; y

$\mathrm{H}=$ (M2 U M2) U2 (M2 U M2), 2.7.2.

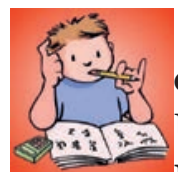

Actividad para el lector: Semejanza de las dos diagonales no principales.

Usando el cubo de Rubik comprobar que $\mathrm{D}_{\mathrm{NP2}} \sim \mathrm{D}_{\mathrm{NP1}}$ $\mathrm{U} 2\left(\cong \mathrm{D}_{\mathrm{NP1}}\right)$.

Y luego verificar

$$
\begin{aligned}
& \hat{c}\left(D_{N P 1}\right) \text { U2 } \hat{d}\left(D_{N P 2}\right) \underset{U 2 ~ R D}{\longrightarrow} C_{I} \\
& \hat{c}\left(D_{N P 2}\right) \text { U2 } \hat{d}\left(D_{N P 1}\right) \underset{U 2 ~ R D}{\longrightarrow} c_{I}
\end{aligned}
$$

donde

$$
\begin{aligned}
& \hat{c}\left(D_{N P 1}\right)=\left(F^{\prime} F^{\prime} R\right. \text { U2 F R' F' R) (U' R U' R'), 12.3.1. } \\
& \hat{d}\left(D_{N P 1}\right)=(R \text { U R' U) (R' F R F' U2 R' F R F') } \\
& \hat{c}\left(D_{N P 2}\right)=M^{\prime} \text { (UR U R'U') M (R'F R F'), 12.7.1. }
\end{aligned}
$$




$$
\begin{aligned}
& \hat{d}\left(D_{N P 2}\right)=(F \text { R' F' R) M' (UR U' R'U') M } \\
& R_{D}=R^{\prime} \text { U2 R U2 R' FRU R' U'R' F' R2 U', 6.1.1. }
\end{aligned}
$$

\subsection{2 $\mathrm{V}_{2}=\mathrm{V}(1,2,1,2)=$ Camaleón $+\mathrm{U}^{\prime}+\mathrm{X}$ :}

La segunda V (Inversa de la Segunda Diagonal NO Principal)

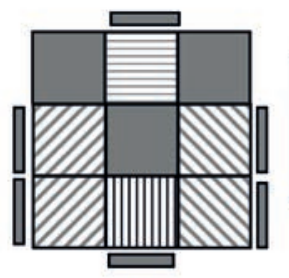

$$
\begin{aligned}
& \hat{c}\left(V_{2}\right)=\hat{d}\left(D_{N P 2}\right)=(\text { F R' F' R) M' (UR U' R'U') } M \\
& d\left(V_{2}\right)=\hat{d}(X) U^{\prime} \text { d(Camaleón) }
\end{aligned}
$$

$\mathbf{V}_{2}$

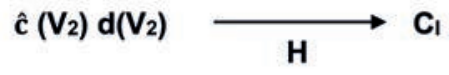

donde $\hat{d}(\mathrm{X})=\hat{\mathrm{c}}(\mathrm{X})=(\mathrm{MU})_{4}\left(\mathrm{M}^{\prime} \mathrm{U}\right)_{4}$;

d̂ (Camaleón) = (r U R' U') (r' F R F'), 7.6.1; y

$\mathrm{H}=$ (M2 U M2) U2 (M2 U M2), 2.7.2.

Actividad para el lector: La Simétrica de la segunda V y su semejanza con ésta

Usando el cubo de Rubik comprobar que $\mathbf{V}_{2}{ }^{\mathrm{s}} \sim \mathrm{V}_{2}$ con

$$
\hat{\mathbf{c}}\left(\mathbf{V}_{2} \mathrm{~s}^{\mathrm{S}}\right)=\left(\mathbf{F}^{\prime} \mathbf{L} \mathbf{F} \mathbf{L}^{\prime}\right) \mathbf{M}^{\prime}\left(\mathbf{U}^{\prime} \mathbf{L}^{\prime} \mathbf{U}\right. \text { LU) M. }
$$

$\mathrm{Y}$ luego verificar

$$
\begin{aligned}
& \hat{c}\left(V_{2} s\right) \hat{d}\left(V_{2}\right) \underset{E U 2}{\longrightarrow} C_{1}
\end{aligned}
$$

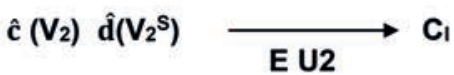

donde

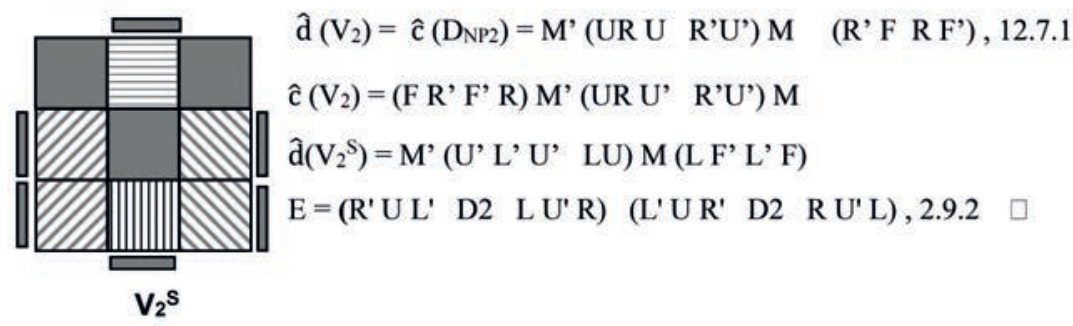


Síntesis tabular OLL “Canónica” (12.5 $\rightarrow 12.7$

\begin{tabular}{|c|c|c|}
\hline \begin{tabular}{l|}
12.5 .1 \\
SMDIP $_{1}$
\end{tabular} & 㥩 & 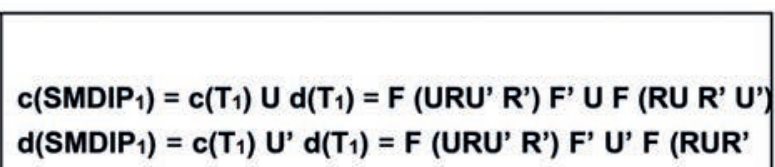 \\
\hline \begin{tabular}{|l|}
12.6 .1 \\
SMDIP $_{2}$
\end{tabular} & & $\begin{array}{l}c\left(S_{M D I P}\right)=d\left(S_{M D I P}\right) U^{\prime}=c\left(T_{1}\right) U^{\prime} d\left(T_{1}\right) U^{\prime} \\
d\left(S_{D D} P_{2}\right)=U c\left(S^{\prime} I_{1}\right)=U c\left(T_{1}\right) U d\left(T_{1}\right)\end{array}$ \\
\hline $\begin{array}{c}12.7 .2 \\
V_{2}\end{array}$ & & $\begin{array}{l}c\left(V_{2}\right)=\left(F R^{\prime} F^{\prime} R\right) M^{\prime}\left(U R U^{\prime} R^{\prime} U^{\prime}\right) M \\
d\left(V_{2}\right)=M^{\prime}\left(U R \cup R^{\prime} U^{\prime}\right) M\left(R^{\prime} F R F^{\prime}\right) L^{\prime}\end{array}$ \\
\hline
\end{tabular}

Las inversas de esas tres configuraciones

\begin{tabular}{|c|c|c|}
\hline & Inversa & Constructor/deconstructor de la Inversa \\
\hline SMDIP $_{1}$ & $\begin{array}{l}\text { SMDIP }_{1}^{-1} \\
\left(\sim \text { SMDIP }_{2}\right. \\
)^{\prime}\end{array}$ & 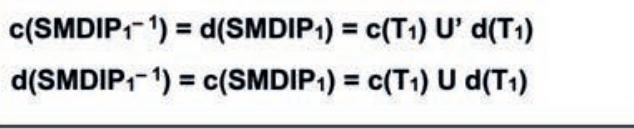 \\
\hline SMDIP $_{2}$ & $\begin{array}{c}\text { SMDIP }_{2}-1 \\
\left(\sim \text { SMDIP }_{1}\right. \\
)\end{array}$ & $\begin{array}{l}c\left(S_{M D I P}{ }^{-1}\right)=d\left(S_{M D I P}\right)=U c\left(T_{1}\right) U d\left(T_{1}\right) \\
d\left(S_{M D I P}{ }^{-1}\right)=c\left(S_{D D I P}\right)=c\left(T_{1}\right) U^{\prime} d\left(T_{1}\right) U^{\prime}\end{array}$ \\
\hline $\mathbf{V}_{\mathbf{2}}$ & $\begin{array}{c}D_{N P 2} \\
\left(\sim D_{N P 1}\right)\end{array}$ & $\begin{array}{l}c\left(D_{N P 2}\right)=d\left(V_{2}\right)=M^{\prime}\left(U R U R^{\prime} U^{\prime}\right) M\left(R^{\prime} F R F^{\prime}\right) \\
d\left(D_{N P 2}\right)=c\left(V_{2}\right)=\left(F R^{\prime} F^{\prime} R\right) M^{\prime}\left(U R U^{\prime} R^{\prime} U^{\prime}\right) M\end{array}$ \\
\hline
\end{tabular}

La semejanza $(\sim)$ acá es con una congruente a la configuración allí nominada

Recordar: Las configuraciones T, Mini $\mathrm{J}_{1}$, Mini $\mathrm{L}_{1}$ y Las cruces ampliadas OLL, junto con los 21 PLL ya conocidos y las rápidas deconstrucciones mencionadas en los últimos cuatro capítulos constituyen. 
El "Método reducido de Fridrich" para la solución de la Última capa del cubo de Rubik

12.8 Las "Configuraciones Puntuales"47 a partir de las "Cruces ampliadas"

\begin{tabular}{|c|c|}
\hline Configuración OLL & "Cruz Ampliada" + Uk + $\mathrm{mJ}_{1}+\mathrm{Un}$ \\
\hline $\begin{array}{ll}12.6 .1 & \text { SMDIP }_{2}\end{array}$ & $\mathrm{Pez}_{R}+\mathrm{X}$ \\
\hline SMDIP $_{1}$ & $\mathrm{PeZL}_{\mathrm{e}}+\mathrm{U}+\mathrm{X}$ \\
\hline Punto1 & $\mathrm{Cruz}_{1}+U+X$ \\
\hline Punto2 & $\mathrm{Cruz}_{2}+\mathrm{U} 2+\mathrm{X}$ \\
\hline 12.7 .2 & Camaleón + U' + X \\
\hline 12.3 .2 & Botella $+X$ \\
\hline 12.3 .1 & Pajarita + U2 + X \\
\hline
\end{tabular}

47 Excepto la "X". 
El presente libro realiza un compendio de los resultados del análisis matemático de los movimientos y algoritmos, tanto OLL(Orientation of Last Layer: Orientación de la última capa), como PLL(Permutation of Last Layer: Permutación de la última capa), realizados sobre la última capa en el cubo de

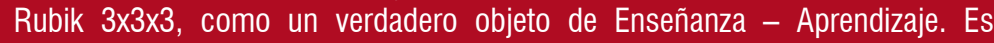
resultado de una investigación aplicada en estudiantes de la Licenciatura de Matemáticas, como alternativa didáctica en asignaturas como Teoría de Grupos, Teoría de Números y Matemáticas Recreativas, que correspondieron a la búsqueda de información, desarrollo intuitivo, formalización, pruebas de campo con los estudiantes y recolección de resultados entre otros.

La presente obra inicia en el capítulo uno con un recorrido por los elementos básicos del cubo de Rubik, el cual es un rompecabezas mecánico, inventado por el escultor y arquitecto húngaro Ernő Rubik en el año 1974; en este capítulo se describen conceptos como el de cara, centro, vértice, arista, torsión y los movimientos básicos, con la respectiva notación, este capítulo finaliza con la construcción y análisis del primer OLL, denominado la T 1, su inverso, su simétrico y el inverso del simétrico. El capítulo 2 se analiza la segunda $T$ (denominada T 2 ) y los primeros 7 PLL. El capítulo 3 y 4 se refieren a las operaciones, configuraciones semejantes y potencias de algunos OLL/PLL. Los capítulos 5 al 12 hacen referencia a la construcción de OLL/PLL puntuales donde se resaltan versiones básicas y ampliadas de las Mini J, las Mini L, las Cruces, las barras centrales además de las 4G, Los Peces Básicos y los puntos. 